University of Louisville

ThinkIR: The University of Louisville's Institutional Repository

Electronic Theses and Dissertations

$8-2020$

\title{
Tissue-resident myeloid-derived suppressor cells modulate immune homeostasis in healthy adipose.
}

Katlin Brooke Stivers

University of Louisville

Follow this and additional works at: https://ir.library.louisville.edu/etd

Part of the Biochemical Phenomena, Metabolism, and Nutrition Commons, Biological Phenomena,

Cell Phenomena, and Immunity Commons, Cell Biology Commons, and the Immunology and Infectious

Disease Commons

\section{Recommended Citation}

Stivers, Katlin Brooke, "Tissue-resident myeloid-derived suppressor cells modulate immune homeostasis in healthy adipose." (2020). Electronic Theses and Dissertations. Paper 3502.

https://doi.org/10.18297/etd/3502

This Doctoral Dissertation is brought to you for free and open access by ThinkIR: The University of Louisville's Institutional Repository. It has been accepted for inclusion in Electronic Theses and Dissertations by an authorized administrator of ThinkIR: The University of Louisville's Institutional Repository. This title appears here courtesy of the author, who has retained all other copyrights. For more information, please contact thinkir@louisville.edu. 


\title{
TISSUE-RESIDENT MYELOID-DERIVED SUPPRESSOR CELLS MODULATE IMMUNE HOMEOSTASIS IN HEALTHY ADIPOSE
}

\author{
By \\ Katlin Brooke Stivers \\ B.A., Wittenberg University, 2013 \\ M.S., University of Louisville, 2016 \\ A Dissertation \\ Submitted to the Faculty of the \\ School of Medicine of the University of Louisville \\ in Partial Fulfillment of the Requirements \\ for the Degree of \\ Doctor of Philosophy \\ in Microbiolog and Immunology \\ Department of Microbiology and Immunology \\ University of Louisville \\ Louisville, $\mathrm{KY}$
}

August 2020 
Copyright 2020 by Katlin Brooke Stivers

All rights reserved 



\title{
TISSUE-RESIDENT MYELOID-DERIVED SUPPRESSOR CELLS MODULATE IMMUNE HOMEOSTASIS IN HEALTHY ADIPOSE
}

\author{
By
}

\author{
Katlin Brooke Stivers
}

B.A. Wittenberg University, 2013

M.S. University of Louisville, 2016

A Dissertation Approved on

June $10^{\text {th }}, 2020$

by the following Dissertation Committee:

James B Hoying, Ph.D.
Dissertation Director

Pascale Alard, Ph.D.

Dissertation Co-Director

Thomas Mitchell, Ph.D.

Jun Yan, M.D., Ph.D.

Amanda LeBlanc, Ph.D. 


\section{ABSTRACT \\ TISSUE-RESIDENT MYELOID-DERIVED SUPPRESSOR CELLS MODULATE IMMUNE HOMEOSTASIS IN HEALTHY ADIPOSE \\ Katlin B. Stivers \\ June $10^{\text {th }}, 2020$}

Our goal with this study was to gain insight into the homeostatic immune cell network in healthy adipose tissue. Specifically, we sought to determine if the immature myeloid cells found in healthy and inflamed adipose were, in fact, tissueresident myeloid-derived suppressor cells (MDSCs). Using various in vitro and in vivo methods, we have uncovered a population of CD11 $\mathrm{b}^{\mathrm{Hi}} \mathrm{Ly} 6 \mathrm{C}^{\mathrm{Hi}} \mathrm{Ly6G}-\mathrm{MDSCs}$ resident in healthy adipose tissue. To the best of our knowledge this is the first time that these cells have been investigated and described in healthy adipose tissue. Systemic MDSC depletion lead to the accumulation of $\mathrm{CD} 4^{+} \mathrm{CD} 44^{+} \mathrm{CD} 2 \mathrm{~L}^{-}$ TEM in the adipose of otherwise healthy mice and altered the surface markers expressed by adipose tissue-resident macrophages. Furthermore, we found that losing MDSCs significantly changed the transcription levels of multiple immunomodulatory genes in the adipose stromal cell compartment and whole adipose tissue. Taken together, this suggests that adipose-resident MDSCs are one of the key players in the adipose tissue immune cell network, preventing 
aberrant, inflammatory TEM proliferation and modulating the suppressive phenotype of adipose tissue-resident macrophages. 


\section{TABLE OF CONTENTS}

SECTION

PAGE

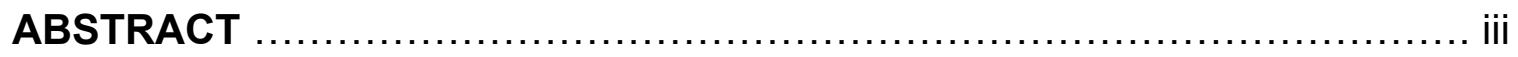

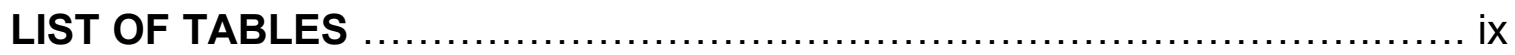

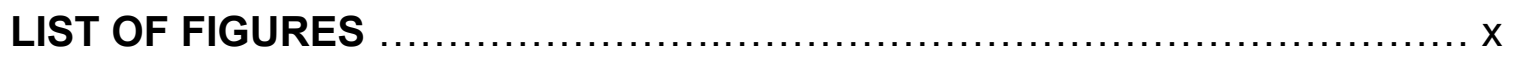

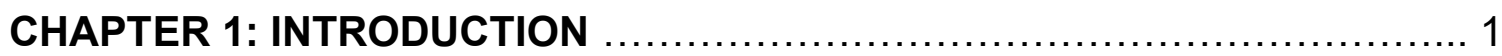

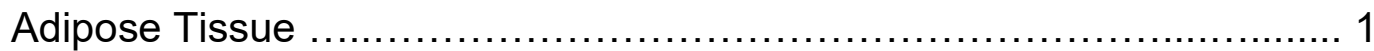

The Essential Role of Inflammation in Healthy Adipose

Tissue Homeostasis .............................................. 2

Adipose-Resident Immune Cell Network ......................... 4

Immunosuppressive Abilities of SVF ............................ 9

Myeloid-derived Suppressor Cells ...................................... 11

MDSCs: An Overview ......................................... 11

MDSC Effector Mechanisms .................................... 13

Nutrient Deprivation ................................... 14

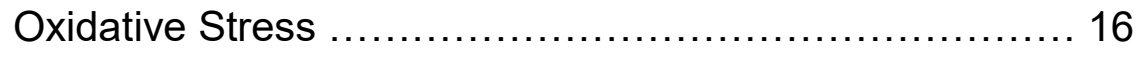

Cytokines \& Direct Cell - Cell Interactions ................ 17

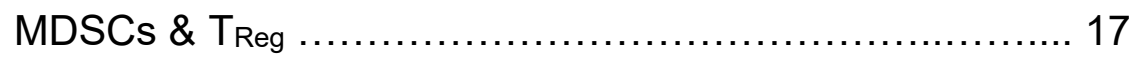

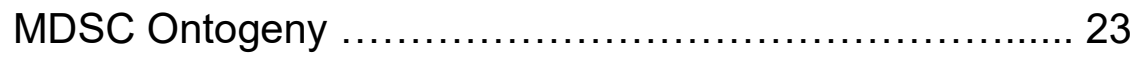

MDSCs in Healthy Tissues ................................ 28 
The MDSC-inducing Potential of Healthy Adipose Tissue 30

CHAPTER 2: METHODS \& MATERIALS 34

Animals ....................................................................... 34

Stromal Vascular Fraction Isolation ....................................... 34

Flow Cytometry Staining for Analysis ................................ 35

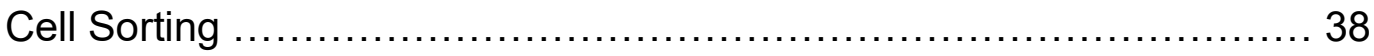

T Cell Proliferation/Suppression Assays ............................... 41

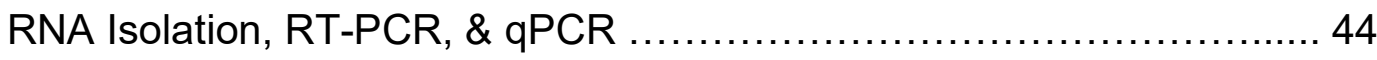

In Vivo MDSC Depletion ................................................... 46

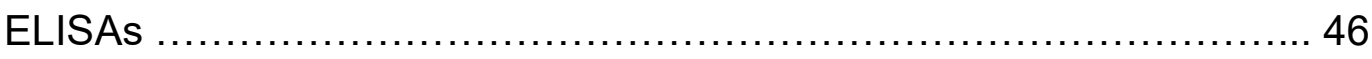

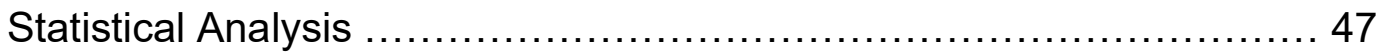

\section{CHAPTER 3: EXPERIMENTAL CHALLENGES \&}

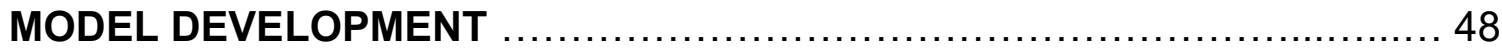

T Cell Proliferation Assays: Adaptations for Working with

Small Cell Populations ................................................. 48

Accommodations for the Unique Nature of Adipose Stromal Cells ....... 58

Flow Cytometry Gating Strategies ............................. 58

Adjusting for Adipose Autofluorescence ..........................63 63

\section{CHAPTER 4: BONA FIDE MYELOID-DERIVED SUPPRESSOR CELLS IN}

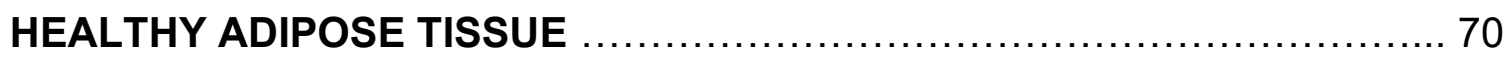

There are CD11 b+ Ly6C HilLow Ly6G ${ }^{+/-}$CD301- cells in healthy adipose tissue 
Adipose-resident CD11 $\mathrm{b}^{+}$Ly6C $\mathrm{C}^{\text {Hi }}$ Ly6G- cells express canonical MDSC-associated genes 74

CD11 $\mathrm{b}^{+}$Ly6 $\mathrm{C}^{\mathrm{Hi}}$ Ly6G- adipose tissue cells suppress

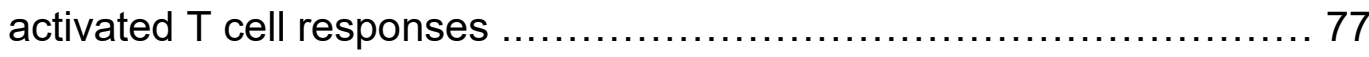

Potential aMDSC Suppression Mechanisms ............................ 81

Apoptosis Induction ............................................ 84

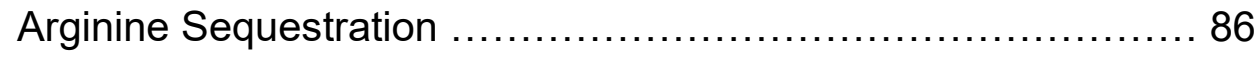

Immunosuppressive Cytokines .................................. 89

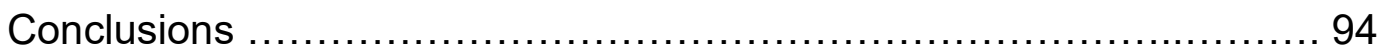

\section{CHAPTER 5: MYELOID DERIVED SUPPRESSOR CELLS REGULATE T CELL NUMBERS \& ADIPOSE MACROPHAGE POLARIZATION}

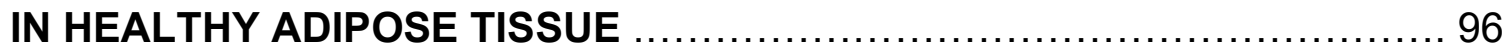

Gr-1 Model of MDSC Depletion ..................................... 96

aGr1 effectively removes cells from spleens \& adipose after 1 week of treatment

Adiposity increases when MDSCs are removed from adipose tissue ...114

Both acute \& chronic MDSC depletion increases the number

of $T$ cells in healthy adipose

The accumulated $\mathrm{T}$ cells are effector memory $\mathrm{T}$ cells

TEMS upregulate expression of markers associated with activation \& proliferation

Adipose-resident macrophages develop a "mixed" phenotype following MDSC depletion 129

ATM gene expression is unaffected by MDSC depletion 135

Adipose-resident MDSCs regulate whole adipose homeostasis \& immune cell dynamics 
Conclusions

CHAPTER 6: DISCUSSION \& FUTURE DIRECTIONS

MDSCs as Part of the Adipose Tissue Immune Cell Network

T Cell Dynamics

Macrophage Dynamics 146

Trials \& Tribulations of Working with the aGr1 Model of MDSC Depletion 150

Sexual Dimorphism in Healthy Adipose Tissue Immune Homeostasis 152

Translational \& Therapeutic Potential 160

FUTURE DIRECTIONS 165 How do adipose-resident MDSCs prevent CD4 ${ }^{+} \mathrm{T}_{\mathrm{EM}}$ proliferation under healthy conditions? 165

What recruits MDSCs to healthy adipose tissue? 168

What is the relationship between MDSCs \& metabolic disease? 


\section{LIST OF TABLES}

TABLE

PAGE

1.1: Brontë et al (2016) Algorithm for Characterizing MDSCs .................. 13

1.2: Similarities Between the Tumor \& Adipose Microenvironments ............. 33

2.1: List of Antibodies Used in Flow Cytometry Panels .......................... 37

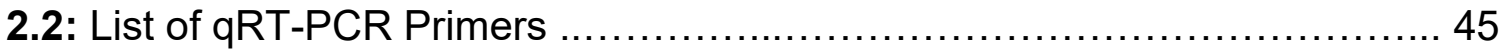

3.1: Conditional T Cell Activation Algorithm for Co-cultures ...................... 50 


\section{LIST OF FIGURES}

FIGURE

PAGE

1.1: Homeostatic Immune Cell Network in Healthy Adipose Tissue ............... 8

1.2: MDSC-mediated nutrient deprivation downregulates activated

$T$ cell proliferation

1.3: MDSCs use oxidative stress responses to prevent $T$ cell activation and subsequent proliferation

1.4: MDSCs disrupt lymphocyte trafficking into and out of the tumor

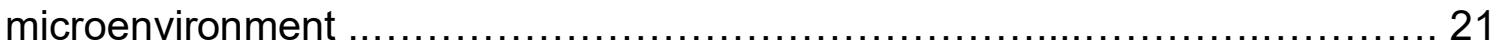

1.5: Immunomodulatory effects of MDSCs on innate immune cells .............. 22

1.6: Candidate signaling cascades contributing to MDSC accumulation and suppressive phenotype ........................................................ 27

2.1: Cell sorting strategies for FACS isolation of SVF subpopulations ........... 39

2.2: FACS isolated aMDSCs require a brief rest period before use in proliferation

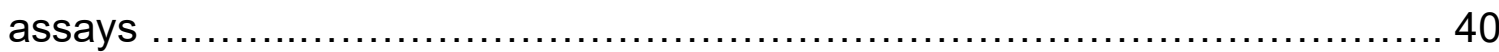

2.3: Schematic of TetraZ-based MTT assay workflow .......................... 43

3.1: CFSE can be used to measure the proliferation of as few as 2500 MACSenriched T cells ................................................................. 56

3.2: MDSCs suppress aCD3/aCD28-stimulated proliferation of splenocytes and MACS-enriched T cells ....................................................... 57

3.3: SVF Myeloid Cell Gating Strategy .......................................6 60

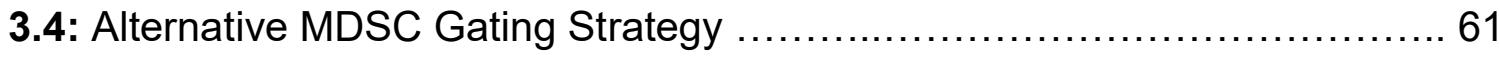

3.5: T Cell Phenotype \& Activation State Gating Strategy ..................... 62

3.6: Flow cytometry analysis of SVF requires manually adjusting spectral overlap values to account for the natural autofluorescence of AT 
4.1: CD11 $b^{+}$CD301- Ly6CHilLow Ly6G $^{+/-}$cells are in adipose tissue from metabolically healthy mice

4.2: PMN-MDSCs make up the majority of $L y 6 G^{+}$cells in healthy adipose tissue 73

4.3: Gene expression in Sorted CD11b ${ }^{+}$CD301- Ly6CHi Ly6G- cells 76

4.4: Sorted CD11 $b^{+}$CD301- Ly6C ${ }^{H i}$ Ly6G- cells suppress aCD3/aCD28 activated $T$ cell proliferation

4.5: Compared to controls, co-culture with aMDSCs reduces the overall number of $\mathrm{T}$ cells, without impeding their initial activation 83

4.6: aMDSCs modulate T cell proliferation without inducing apoptosis 85

4.7: aMDSCs do not rely on arginase-mediated nutrient deprivation to suppress $\mathrm{T}$ cell proliferation 88

4.8: IL-10 concentrations were below detection in aMDSC co-cultures due to small number of cells in each well

4.9: aMDSCs may modulate immune cell activity via TGF $\beta$ production 93

5.1: Healthy adipose tissue contains very few of the non-MDSC cells affected by treatment with $\alpha \mathrm{Gr} 1$ 104

5.2: Pooling adipose tissue for analysis masks differences in treatment efficacy between individual animals

5.3: Analyzing AT SVF from individuals reveals cage-dependent variations in immune cell population distribution 106

5.4: $\alpha \mathrm{Gr} 1$ antibody treatment efficiently depletes splenic MDSCs 110

5.5: $\alpha \mathrm{Gr} 1$ antibody treatment efficiently removes MDSCs from epididymal fat pads

5.6: Generic antibody treatment does not affect the adipose-resident MDSC population

5.7: Ly6G+ SVF cells are efficiently depleted following treatment with $\alpha \mathrm{Gr} 1$ antibody 
5.8: aMDSC depletion does not affect superficial measures of metabolic health or the cellularity of adipose tissue

5.9: MDSC depletion in healthy adipose tissue results in increased numbers of $T$ cells

5.10: $\alpha \mathrm{Gr} 1$ treatment has differential effects on splenic $\mathrm{T}$ cell populations ..... 119

5.11: Accumulated $T$ cells in adipose following $\alpha \mathrm{Gr} 1$ treatment are effector memory $T$ cells

5.12: The splenic $C D 4^{+} \mathrm{CD} 44^{+} \mathrm{CD} 62 \mathrm{~L}^{-} \mathrm{T}_{\mathrm{EM}}$ population is unaffected by systemic MDSC depletion

5.13: $\alpha \mathrm{Gr} 1$ treatment, but not IgG2b treatment, increases CD69 expression on CD4+ TEM in adipose SVF

5.14: $\mathrm{CD} 4+\mathrm{T}$ cells in the adipose increase their expression of $\mathrm{Ki}-67$ following acute MDSC depletion

5.15: CD4+ T cells may upregulate IFNY following MDSC depletion 128

5.16: $\alpha \mathrm{Gr} 1$ treatment does not affect the number or proportion of adipose tissue-resident macrophages

5.17: ATM surface marker expression is altered by acute MDSC depletion ... 133

5.18: ATMs differentially respond to acute and chronic MDSC depletion

5.19: ATM expression of immunomodulatory genes remains unchanged following $\alpha \mathrm{Gr} 1$ treatment

5.20: Adipose-resident MDSCs regulate whole tissue homeostasis as well as adipose immune cell dynamics

6.1: The Role of aMDSCs in Healthy Adipose Tissue 149

6.2: $\alpha \mathrm{Gr} 1$ antibody treatment efficiently depletes MDSCs from adipose tissue in female mice 157

6.3: MDSC depletion increases the number of T cells in healthy female adipose tissue

6.4: $\alpha \mathrm{Gr} 1$ treatment augments the number of macrophages and changes their surface marker expression in female adipose tissue 159 
6.5: Flow cytometric characterization of healthy adipose tissue from Balb/c mice 163

6.6: Flow cytometric characterization of healthy human adipose tissue 164

6.7: Macrophages, but not T cells have nitrosylated surface proteins in healthy adipose SVF 167

6.8: Proposed model for MDSC recruitment into healthy adipose tissue 169 


\section{CHAPTER 1: INTRODUCTION}

\section{Adipose Tissue}

Adipose tissue (AT) is a loose connective tissue that serves as an energy storage depot, using fatty acid oxidation reactions to store excess glucose and, when necessary, release it back into circulation. This tissue has a multitude of other endocrine, metabolic, and thermoregulatory functions. In mammals, adipose depots are distributed throughout the body and are divided into two main categories: brown and white. Mainly found in infants, brown AT (BAT) uses stored lipids to produce heat and regulate body temperature. In adults the majority of depots are white adipose tissue (WAT) which can also contribute to thermoregulation by developing a "beige" phenotype [1]. For the most part however, WAT stores and releases energy for systemic use in response to changes in insulin and glucagon levels, respectively.

While adipocytes are the main cellular unit of AT, they are only $20-40 \%$ of the total cells found in the tissue, with adipose stromal cells accounting for the remaining $60-80 \%$ of cells [2]. In lean, healthy individuals this stromal-vascular cellular fraction (SVF) contains a wide-array of endothelial cells, mesenchymal stem cells, pre-adipocytes, and immune cells. Altogether they are integral regulators of adipose tissue homeostasis. SVF cells promote angiogenesis; release AT-modulating molecular factors such as cytokines and hormones; and produce the extracellular matrix elements necessary to maintaining tissue integrity. 
The exact distribution of these cell types differs between adipose depots and may depend upon its physiological function [3-8]. Compared to visceral AT depots, the omental AT appears to function more as a tertiary lymphoid tissue than it does an energy storage depot. For instance, special fat-associated lymphoid clusters in omental AT act almost as loosely organized germinal centers, where tissue resident dendritic cells induce antigen-specific B cell responses [9-12]. Over the last 20 years, we have begun to recognize the importance of the SVF immune cell compartment in not only driving obesity-induced visceral AT inflammation, but also maintaining in modulating the day-to-day functions of this tissue [2].

\section{The Essential Role of Inflammation in Healthy Adipose Tissue Homeostasis}

Catering to the metabolic needs of an organism is a dynamic process. As blood glucose levels increase or decrease, adipocytes initiate lipogenesis or lipolysis reactions to sequester or release energy, respectively. Accompanying the ebb and flow of lipids being stored or released are requisite fluctuations in the physical size of adipocytes. Practically, this calls for near constant extracellular matrix (ECM) remodeling in the adipose to accommodate these physical changes. Importantly, Wernstedt Asterholm, Tao [13] (2014) found that as adipocytes initiated either of these metabolic reactions, they simultaneously upregulated their expression of TNFa. The subsequent inflammatory cascade eventually precipitated the breakdown of the surrounding ECM, allowing adipocytes to expand or contract as necessary. This finding was surprising given that this cascade was observed in healthy, chow-fed mice and that both TNFa upregulation and AT inflammation are typically associated with obesity. Furthermore, an 
adipocyte specific inhibition of TLR4-, TNFa-, and IL1 $\beta$-mediated signaling led to significant metabolic dysfunction in otherwise healthy animals and enhanced the metabolic consequences of a high-fat diet compared to WT controls. Implicating that this inflammatory pathway is physiologically necessary for the maintenance of healthy adipose tissue homeostasis [13].

Further complicating our perception of inflammatory dynamics in AT is the ambiguous role of TNFR1 signaling in metabolic health and disease [14]. For instance, transgenic knockout of TNFR1 gained significantly more weight on a normal diet than wild-type controls confirming the findings from the aforementioned study concerning the integral nature of TNF-mediated signaling in maintaining healthy metabolic homeostasis. When fed a high-carbohydrate diet however, these TNFR1 knockout mice had improved insulin sensitivity and had reduced adiposity compared to wild-type controls on the same diet, highlighting the deleterious effects this same signaling pathway can have in a pathological setting [14]. Altogether, these studies have brought to light the important role that acute inflammation, as reflected by TNFa signaling, plays in the day-to-day functions of this dynamic tissue and calls for a reassessment of the disease-centric way in which AT inflammation is most often viewed.

Besides the requirement for TNF- $\alpha$ mediated signaling, ECM remodeling could also be a constitutive source of toll-like receptor (TLR) ligands in the tissue $[15,16]$. By-products of ECM degradation have the potential to be both pro- and anti-inflammatory, depending upon the microenvironmental context and through which TLRs they mediate their signals [15]. In AT specifically, these molecules are 
associated with the obesity-induced inflammation [17, 18]. However, even under normal conditions, AT is constantly being degraded and remodeled, producing inflammatory-like conditions, yet healthy tissue is not considered to be chronically inflamed. What prevents these pro-inflammatory molecules and the signaling pathway involved in AT remodeling from developing into the pathological inflammation observed in metabolic diseases?

\section{Adipose-Resident Immune Cell Network}

According to Wernstedt-Asterholm et al $[13,19]$, "immunological fitness" is the saving grace of this tissue. Immunological fitness is a measure of an organism's (or tissue's) ability to efficiently mount and subsequently resolve an effective immune response. In this case it applies to the acute TNFa-mediated inflammatory response required for ECM remodeling in healthy AT. If this immunological fitness is compromised, then the tissue is no longer able to cope with these acute inflammatory responses and develops metabolic complications, as demonstrated by the various transgenic knockout models used in this study [13].

Going one step further, AT immunological fitness is, at its core, a function of the tissue-resident immune cell network. This network is inherently immunomodulatory in healthy AT and most likely evolved as a means to efficiently quell the intermittent acute inflammatory responses required to properly accommodate lipid storage and release by adipocytes. Adipose tissue-resident macrophages (ATMs) make up the largest percentage of these cells by far [20, 21]. ATMs are considered true "tissue-resident" macrophages as they are fetal 
liver derived [22], do not develop from circulating monocytes, and the population undergoes self-renewing in situ proliferation [23].

Classically, murine ATMs are defined as $\mathrm{F} 4 / 80^{+} \mathrm{CD} 301^{+} \mathrm{CD}^{2} 06^{+} \mathrm{CD} 11 \mathrm{C}^{-}$ CD64- and exhibit transcriptional and functional traits associated with "M2-like" polarization. Recently, the boundaries of this definition have expanded as more comprehensive, single-cell level studies have uncovered heterogeneity within this macrophage compartment in healthy AT [24, 25]. Finding surface marker heterogeneity among this population is not that surprising considering the extreme sensitivity of macrophages to microenvironmental cues and the dynamic AT microenvironment. On the other hand, their functional heterogeneity within the same tissue is unexpected. These cells can perform a multitude of tasks required for AT to function properly, including clearing dead cellular debris and directing ECM remodeling efforts [20]. Specific ATM functions may also be dependent upon their location within the tissue. One study identified a subset of vasculatureassociated ATMs with enhanced endocytic capacity compared to other ATM subsets [25]. Additionally, ATMs found around hypertrophic adipocytes appear to act as "clean-up crews" phagocytosing excess lipids released by the adipocyte [26, 27]. Similarly, fatty acids released during weight loss-induced lipolysis are taken up and stored by ATMs [28]. A common theme among all ATM subsets however, is that obesity profoundly alters their phenotype and function, steering them towards a more pro-inflammatory, "M1-like" polarization state [20, 21, 29]. Monocyte-derived macrophages also infiltrate the tissue exacerbating and sustaining the pro-inflammatory nature of the obese AT microenvironment [20]. 
ATMs maintain their "M2" status in healthy adipose via cytokine signaling and cross-talk with AT-resident eosinophils and innate lymphoid type 2 cells (ILC2s) [30]. IL-33 signaling recruits and activates ILC 2s into the adipose, where they regulate the immunosuppressive phenotypes of many other SVF cell types [31-33]. Their production of IL-5 and IL-13 is especially important for the resiliency of the ATM phenotype against inflammatory challenge [30, 34]. IL-5 also recruits eosinophils into the tissue. Usually associated with promoting rapid type II allergy responses, these innate granulocytes serve a more regulatory role in adipose. Eosinophils are one of the primary sources of IL-4 in AT, without which ATMs begin to switch towards a more pro-inflammatory "M1" phenotype [30, 35].

Adaptive immune cells also participate in the AT immune cell network in the form of both B and T lymphocytes [2]. The B cell compartment in lean AT is primarily made up of regulatory B cells that produce IL-10 and influence ATM phenotype [36, 37], although not much work has been done to elucidate any additional notes these cells may play in maintaining AT homeostasis.

Much more is known about the T cells found in adipose, albeit through the lens of obesity. For instance, obesity is perpetuated by the infiltration of both $\mathrm{CD} 4^{+}$and $\mathrm{CD}^{+} \mathrm{T}$ cells, with a limited TCR repertoire, into the tissue [38]. There are CD4+ $\mathrm{T}$ cells in lean adipose as well. Their origin, function, and specificity however have yet to be discerned. In mice, depending upon the AT depot, the majority of these cells exhibit a CD44+ ${ }^{+}$CD62L- effector memory phenotype and are capable of mounting a Th1-type response upon non-specific co-stimulation $[39,40]$. While the antigen specificity of these memory $T$ cells is unknown, there is growing evidence 
that the WAT may act as a reservoir for pathogen-specific memory T cells $[38,39$, 41-48]. For example, Toxoplasma gondii and Yersinia pseudotuberculosis-specific effector and tissue-resident memory $\mathrm{T}$ cells were found in adipose [39]. Moreover, these populations were self-sustaining, long-lived, and capable of mounting an effective response upon reinfection. A similar population has also been observed in human adipose, where HIV positive T cells have been identified even in patients with undetectable viral loads in circulation [42].

With aging, in both murine and human adipose, the phenotypic distribution of $\mathrm{CD}^{+} \mathrm{T}$ cells shifts, with the majority transitioning from memory $\mathrm{T}$ cells to regulatory $T$ cells ( $\left.T_{\text {Regs }}\right)[39,49,50]$. $T_{\text {Regs }}$ accumulate in AT with age. For the most part, these cells appear to serve a protective purpose in the tissue by influencing the suppressive phenotypes of other AT immune cells via TGF $\beta$ and IL-10 production [2]. Furthermore, the addition of exogenous $T_{\text {Regs }}$ in obese mice alleviates some of their metabolic symptoms [50]. This may not always be the case however, as systemic ablation of $T_{\text {Regs }}$ actually prevents aging-related metabolic syndromes from developing [49].

Even though our understanding of the healthy adipose immune cell network is relatively limited, it is still clear that these cells play a major role in modulating homeostasis in this tissue and in protecting it from the pro-inflammatory challenges it faces as a consequence of its dynamic physiologic functions (Figure 1.1). 


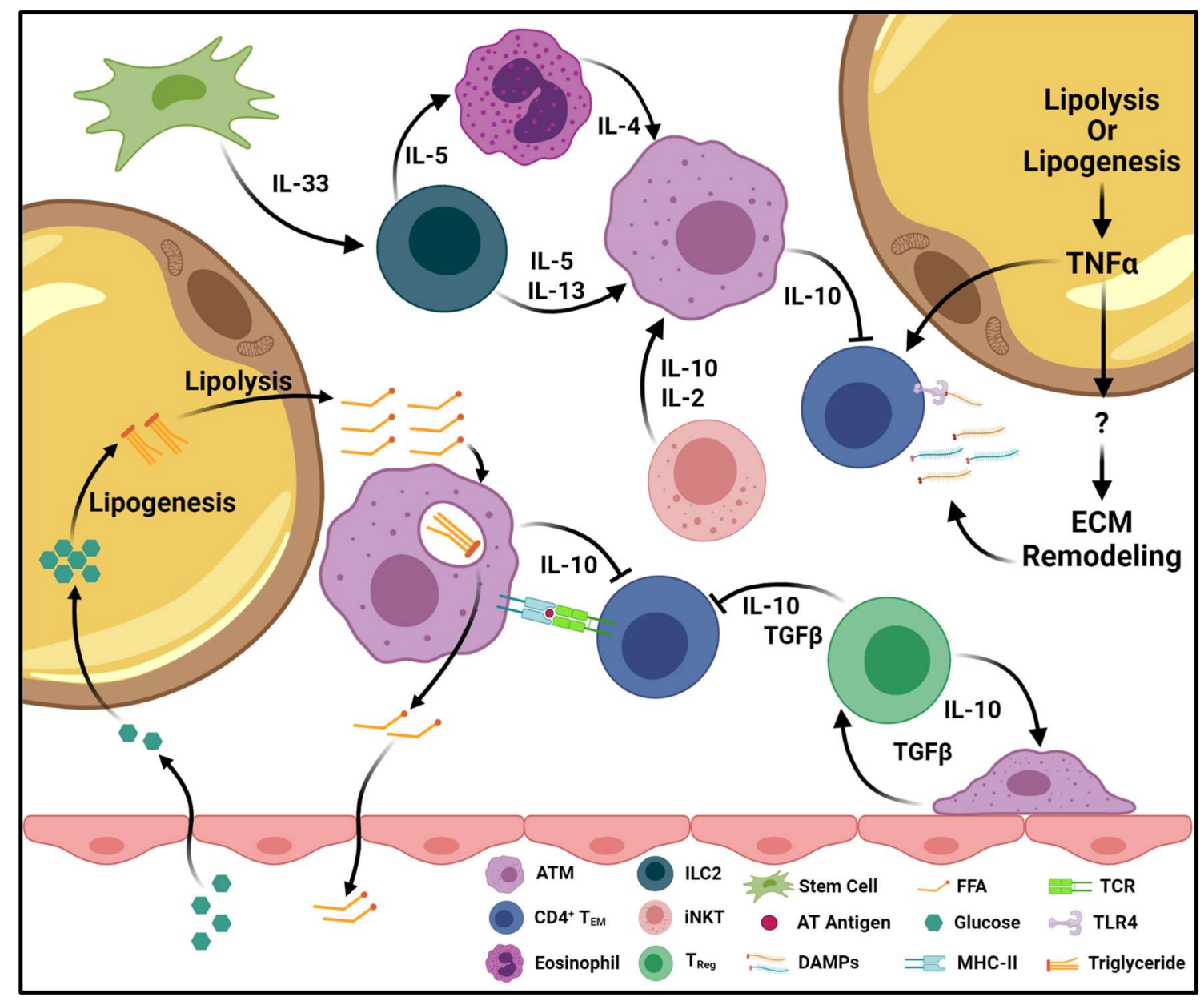

Figure 1.1: Homeostatic Immune Cell Network in Healthy Adipose Tissue. Dynamic immune cell interplay prevents $T$ cell-mediated adipose tissue inflammation under normal dietary pressures. ATM: adipose tissue-resident macrophage. ECM: extracellular matrix. AT: adipose tissue. DAMPs: damageassociated molecular patterns. FFA: free-fatty acids. TCR: T cell receptor. TLR: toll-like receptor. 


\section{Immunosuppressive Abilities of SVF}

The immunomodulatory and tissue remodeling properties of these nonadipocyte cells are currently being investigated for various cell-based antiinflammatory and wound-healing therapeutic applications [51-59]. By studying the impacts SVF-based cellular therapies have on local immune responses we can gain insight into the nature of the immunomodulatory microenvironment that these cells create in their native tissue.

Initially, many of these studies revolved around adipose-derived mesenchymal stem cells (AD-MSCs) which are derived from SVF following several days of culture [60]. Similar to bone marrow-derived mesenchymal stem cells ADMSCs possess potent regenerative and immunomodulatory abilities, but are much more accessible, making them a promising alternative to bone marrow MSC-based treatments [61-68]. In solid organ transplantation, donor-derived AD-MSCs have been shown to suppress activated recipient $T$ cell responses and reduce the severity of acute rejection in rat models of allogeneic liver [69] and kidney [70] transplantation. Additionally, multiple post-transplant injections of donor-derived AD-MSCs have been shown to prolong the survival of a vascularized-composite tissue allograft (VCA) by increasing the number of circulating $T_{\text {Regs }}$ and reducing allograft-infiltrating leukocytes $[64,71]$. These cells have also been associated with the accumulation of immunosuppressive "M2"- like macrophages [72-74]. One study demonstrated that AD-MSCs reduced aortic inflammation in a murine aneurysm model by directly inducing M2 polarization [75]. Other immunomodulatory functions of these cells include suppressing activated B cell production of immunoglobulin [60], as well as preventing dendritic cell (DC) 
differentiation and downregulating their expression of co-stimulatory molecules $[63,67,76]$.

Freshly isolated SVF cells also possess substantial regenerative and immunosuppressive properties, but do not require extensive in vitro enrichment or expansion to be used therapeutically [51-54]. Compared to AD-MSCs, SVF-based therapies are equally as effective at treating inflammation and promoting wound healing [55-57, 59, 77-81]. In a murine model of multiple sclerosis, SVF cells increased Treg recruitment to sites of inflammation to a similar, if not greater, degree than AD-MSCs $[54,55]$. Clinically, SVF has been used to treat systemic sclerosis [82], peripheral vascular disease [58], and, in one case, industrial X-ray injury [83]. In each of these studies, SVF cells reduced circulating levels of inflammatory cytokines and disease symptom severity in general [58, 82, 83]. Recently, clinical trials performed at the University of Louisville have shown promising initial results using freshly isolated, autologous SVF cells as a means to prevent and treat acute VCA rejection [84]. Interestingly, the innate heterogeneity of the SVF isolate makes it difficult to develop a standardized cellular product for regulatory approval. However, this same heterogeneity may also be the very aspect behind the robust therapeutic efficacy of SVF-based treatments as the in vivo crosstalk that promotes their anti-inflammatory properties in adipose likely continues after isolation and may preserve their immunomodulatory character when transplanted into an inflamed microenvironment. 


\section{Myeloid-Derived Suppressor Cells}

In our lab, we focused on developing an SVF-based therapy to improve vascular function following injury. We found that treatment with the SVF improved vasodilation and reduced immune cell infiltration into the wall of the injured vessel [85]. Moreover, the SVF isolate lost many of its beneficial effects when the CD11 $\mathrm{b}^{+}$ cells were depleted. Further flow cytometric characterization of these CD11 $\mathrm{b}^{+}$cells revealed that a portion also expressed Gr1, a marker typically used to identify neutrophils. However, given that the SVF utilized in these experiments was isolated from healthy mice and neutrophil numbers are very low, it is unlikely that these $\mathrm{CD} 11 \mathrm{~b}^{+} \mathrm{Gr} 1^{+}$cells were neutrophils [86]. Another, albeit less conventional, candidate are myeloid-derived suppressor cells (MDSCs).

\section{MDSCs: An Overview}

MDSCs are classically defined as a heterogeneous population of potently immunosuppressive, $\mathrm{CD} 11 \mathrm{~b}^{+} \mathrm{Gr} 1^{+}$immature myeloid cells. MDSCs were first identified as a major contributor to the anti-inflammatory nature of the tumor microenvironment (TME) and have since been described performing similar functions in several chronic inflammatory and autoimmune diseases [87-89]. They have also been shown to promote allogeneic and maternal-fetal tolerance [90-92].

Myeloid-derived suppressor cells (MDSCs) are a heterogeneous collection of "immature", or non-terminally differentiated, $\mathrm{CD} 11 \mathrm{~b}^{+} \mathrm{Gr} 1^{+}$cells that accumulate at sites of chronic inflammation. They can be further classified into two subcategories based on their expression of Ly6C and Ly6G: Polymorphonuclear PMN-MDSCs $\left(C D 11 b^{+}\right.$Ly6C ${ }^{\text {Int/Low }}$ Ly6G $\left.^{+}\right)$and Monocytic M-MDSCs $\left(C D 11 b^{+}\right.$ 


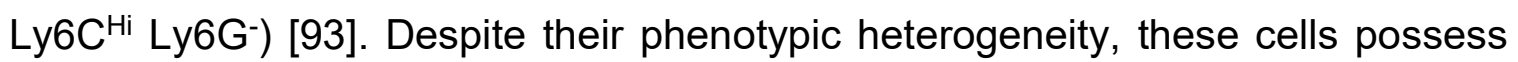
one common trait. They are potently immunosuppressive, targeting both antigenspecific and non-specific inflammatory responses. MDSCs were first identified as one of the primary cell types responsible for the impaired immune surveillance observed in the tumor microenvironment (TME) and have since been described performing similar functions in several chronic inflammatory conditions. As such, MDSCs are almost exclusively associated with disease and the dysfunctional resolution of chronic inflammation [89, 94-99]. Since the majority of studies on MDSCs revolve around the inhibition of tumoricidal responses, the immunosuppressive activity of these cells is often considered to be an abnormal hijacking of the emergency myelopoiesis response that would not develop under healthy, steady-state conditions.

Over the last decade evidence has accumulated supporting the idea that MDSCs may act as the innate immune system's equivalent of regulatory T cells, protecting tissues from the detrimental effects of chronic inflammation [97]. For instance, these cells have been identified in chronic inflammatory conditions such as rheumatoid arthritis, inflammatory bowel disease, and multiple sclerosis [89, 9499]. In each of those settings, MDSC numbers are correlated with improved disease outcomes as they downregulate activated T cell responses, promote "M2" macrophage polarization, and enhance angiogenesis in the inflamed tissue microenvironment. Additionally, these contentious cells were found to play an integral role in successful, full-term pregnancies by establishing and maintaining 
maternal-fetal tolerance. Their tolerogenic activities may also play a role is prolonging graft survival in allogeneic transplantation [90-92].

The diverse nature of both MDSC phenotype and function has made it difficult to develop a universally applicable definition for these cells. In 2016, MDSC researchers developed a characterization algorithm (Table 1.1) to rectify this [100]. This strategy uses a combination of phenotypic, biochemical, and functional traits to differentiate MDSCs from other myeloid cell types such as monocytes and neutrophils.

Table 1.1: Brontë et al (2016) Algorithm for Characterizing MDSCs.

Defining Characteristics of Myeloid-Derived Suppressor Cells Quintessential CD11b ${ }^{+}$Ly6C $^{\text {Hi/Low }}$ Ly6G $^{+/-}$SSC $^{\text {Low }}$ surface marker phenotype

Immune suppressive activity

Expression of characteristic molecular markers: Arginase, iNOS, IL-10, TGF $\beta$, IL-4R

Expansion of the myeloid compartment under pathological conditions.

\section{MDSC Effector Mechanisms}

The primary effector function of these cells is the inhibition of activated T cell proliferation through a variety of direct and indirect mechanisms. However, their influence is not limited to activated T cell responses, as they have also been found to modulate macrophage phenotype, promote angiogenesis, and induce the development of regulatory $T$ cells in the periphery $[93,98]$. 
It's likely that no single MDSC utilizes more than one of these effector mechanisms at any given time, however the heterogeneous nature of the total MDSC population may mean that many of these suppressive activities could be represented within a single TME [101-103]. Moreover, differences between tumor types strongly affects the cell types and soluble proteins found around the tumor

which means that different tumor types elicit different MDSC responses. The immature nature of MDSCs could also contribute to their functional plasticity as they may be able to respond to environmental signals more quickly than a terminally differentiated cell.

\section{MDSC Effector Mechanisms: Nutrient Deprivation}

Pro- and anti- inflammatory responses are often described in terms of signaling cascades or receptor-ligand interactions. MDSCs however have developed a potent, somewhat round-about means with which to suppress proliferative, inflammatory $\mathrm{T}$ cell responses. In order to divide, $\mathrm{T}$ cells must import certain amino acids or their precursors from the local microenvironment. MDSCs can then sequester these resources, preventing $T$ cells from being able to proliferate further. Arginine and cysteine are the primary targets of this insidious siege.

Excessive arginine consumption is one of the most straightforward examples of nutrient deprivation-mediated immune suppression. In the TME, MDSCs upregulate their expression of induced Nitric Oxide Synthase (iNOS) and Arginase 1 (Arg1), both of which use L-arginine (L-Arg) as their primary substrate. The increased activity of these two enzymes eventually depletes L-Arg from the 
surrounding microenvironment. Without access to this essential amino acid, effector T cells around the tumor cannot proliferate and eventually downregulate surface expression of the CD3 $\zeta$ chain required for proper T cell receptor (TCR) signaling in response to antigen recognition [104]. This essentially leaves $T$ cells "blind" to the continued presence of tumor-derived antigens. Eventually, this prolonged lack of antigen-mediated signaling leads to a significant reduction in effector T cell activation and proliferation. L-arginine sequestration plays such a vital role in MDSC-mediated immune suppression that genetic deletion of just one kind of arginine transporter weakened their ability to inhibit activated $T$ cell proliferation in vitro [105]. In vivo CAT2--- mice, which have dysfunctional cationic amino acid transporter and cannot import arginine, also had reduced tumor growth compared to control animals which was attributed to a relatively greater number of tumor-infiltrating lymphocytes and diminished iNOS activity in the MDSCs themselves [105] (Figure 1.2).

Along with arginine deprivation, MDSCs can also significantly diminish the amount of cysteine and cystine available in the TME [106]. T cells lack the $\mathrm{x}_{c}{ }^{-}$ Cystine-Glutamate antiporter $\left(x_{c}^{-}\right)$required to import cystine from extracellular sources. They can, however, acquire cysteine from their local environment via the Alanine-Serine-Cysteine transporter-1 (Asc-1), a transmembrane neutral amino acid transport protein. Once inside the cell, cysteine is reduced to the requisite cystine, essential for continued protein synthesis. In an inflammatory response, antigen presenting cells (APCs) are thought to be the primary source of extracellular cysteine taken up by T cells. MDSCs on the other hand only possess 
$\mathrm{X}_{c}{ }^{-}$and do not export release oxidized cysteine back into their surroundings. Additionally, APC:MDSC crosstalk causes APCs to downregulate expression of Asc-1 hindering their ability to export cysteine. The rapid uptake and consumption of cystine by MDSCs combined with a reduced rate of cysteine export by APCs drastically impacts the amount of cysteine available for use by proliferating, effector $\mathrm{T}$ cells. Without an available pool of this amino acid, activated $\mathrm{T}$ cells downregulate expression of the $\mathrm{CD} 3 \zeta$ chain resulting in proliferative arrest (Figure 1.2).

\section{MDSC Effector Mechanisms: Oxidative Stress}

Apart from the over consumption of L-Arg, MDSCs use iNOS and Arg-1 to manipulate inflammatory immune responses by inducing oxidative stress within the TME (Figure 1.3). Co-expression of these enzymes, along with NADPH oxidase, allows MDSCs to produce reactive oxygen (ROS) and nitrogen species (RNS) that have various suppressive effects [107]. These induce downstream

effects that can be divided into two broad categories: signal interruption and impaired trafficking. MDSC-induced oxidative stress can manifest in several ways since ROS and RNS do anything from directly damaging the tissue to influencing the folding of individual proteins. In terms of T cell-specific impacts, PMN-MDSCproduced hydrogen peroxide reduces $T$ cell expression of the CD3 $\zeta$ chain [108] (Figure 1.3 a), while M-MDSC-produced peroxynitrite incapacitates IL-2 receptors via nitrosylation [109] (Figure 1.3 b). Either way $T$ cell sensitivity to various activation signals is greatly diminished. Similarly, peroxynitrite can interfere with $T$ cell migration by nitrosylating CCL-2 on the surface of $\mathrm{T}$ cells. This alters the 
conformation of this chemokine, effectively preventing the cell from migrating into the tumor itself [110] (Figure $1.4 \mathrm{a})$.

\section{MDSC Effector Mechanisms: Cytokines and Direct Cell-Cell Interactions}

Although Ros and RNS are powerful mediators of immune suppression, they are not the only molecular tools MDSCs have at their disposal. These cells use both surface and soluble proteins to downregulate the inflammatory, antitumor responses of various cell types in the TME. ADAM17 cleaves L-selectin (CD62L) on the surface of $\mathrm{T}$ cells, downregulating its expression and preventing their trafficking to the draining lymph nodes [111] (Figure 1.4 b). MDSCs can also induce T cell apoptosis by expressing Gal9 on their surface, which interacts with TIM3 on T cells [112] (Figure 1.4 b). MDSC-produced cytokines can also directly influence the pro-inflammatory nature of cells in the TME (Figure 1.5). There is evidence that membrane bound TGF- $\beta$ on MDSCs prevents NK cells from mounting tumoricidal responses [113]. MDSCs also produce IL-10 which steer macrophage polarization towards the tumor preserving, immunosuppressive "M2" phenotype [114, 115]. Additionally, IL-10 production by MDSCs reduces TLR4 expression on the surface of dendritic cells (DC). Without this pattern recognition receptor, DCs cannot recognize tumor-induced tissue damage and stop producing IL-12 which would otherwise be used to promote pro-inflammatory, antigenspecific T cell responses [115]. 


\section{MDSC Effector Mechanisms: MDSCs and TRegs}

MDSCs within the TME directly modulate the functional phenotypes of various innate immune cells. This influence extends to adaptive immunity as well, going beyond simply preventing antigen-specific, pro-inflammatory $\mathrm{T}$ cell responses. MDSCs have the ability to activate and induce $T_{\text {Reg }}$ differentiation in the periphery and activate natural $\mathrm{T}_{\text {Reg }}$ already is circulation [107]. The exact mechanisms behind this have yet to be uncovered, but there are several potential candidates. These include the production of cytokines such as IFNy, IL-10, and TGF $\beta$ [116]; CD40-CD40L-mediated cell-cell interactions [117]; and their increased arginase activity [118]. No matter what the mechanism is behind MDSC: $T_{\text {Reg }}$ interactions, it is clear that induction or a clonal expansion of these cells is a powerful tool that MDSCs can use to manipulate anti-tumor responses in their microenvironment.

Despite the ambiguous nature of these cells, one thing remains clear, wherever there is prolonged chronic inflammation or the persistent need for immune tolerance, suppressive myeloid cells accumulate. This strongly supports the idea that these cells are less of a pathological consequence to an immune insult and more of a physiologically normal innate response to prevent further inflammation-induced tissue damage. 


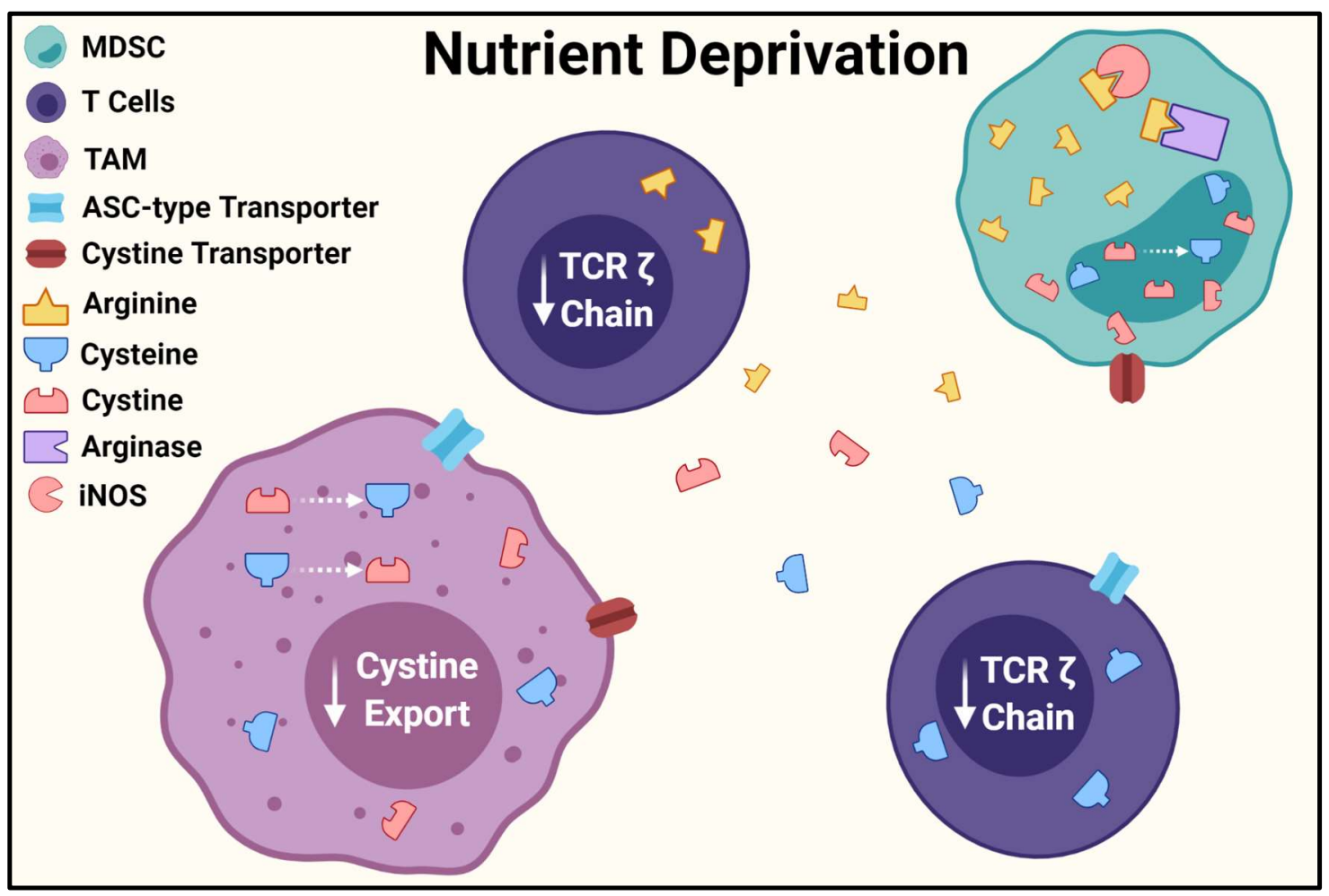

Figure 1.2: MDSC-mediated nutrient deprivation downregulates activated T cell proliferation in the tumor microenvironment. MDSCs disrupt cystine/cysteine homeostasis and overconsume arginine in their local microenvironment, preventing $T$ cells from acquiring these amino acids, essential for their continued proliferation. MDSC: myeloid-derived suppressor cell. TAM: tumor-associated macrophage. 


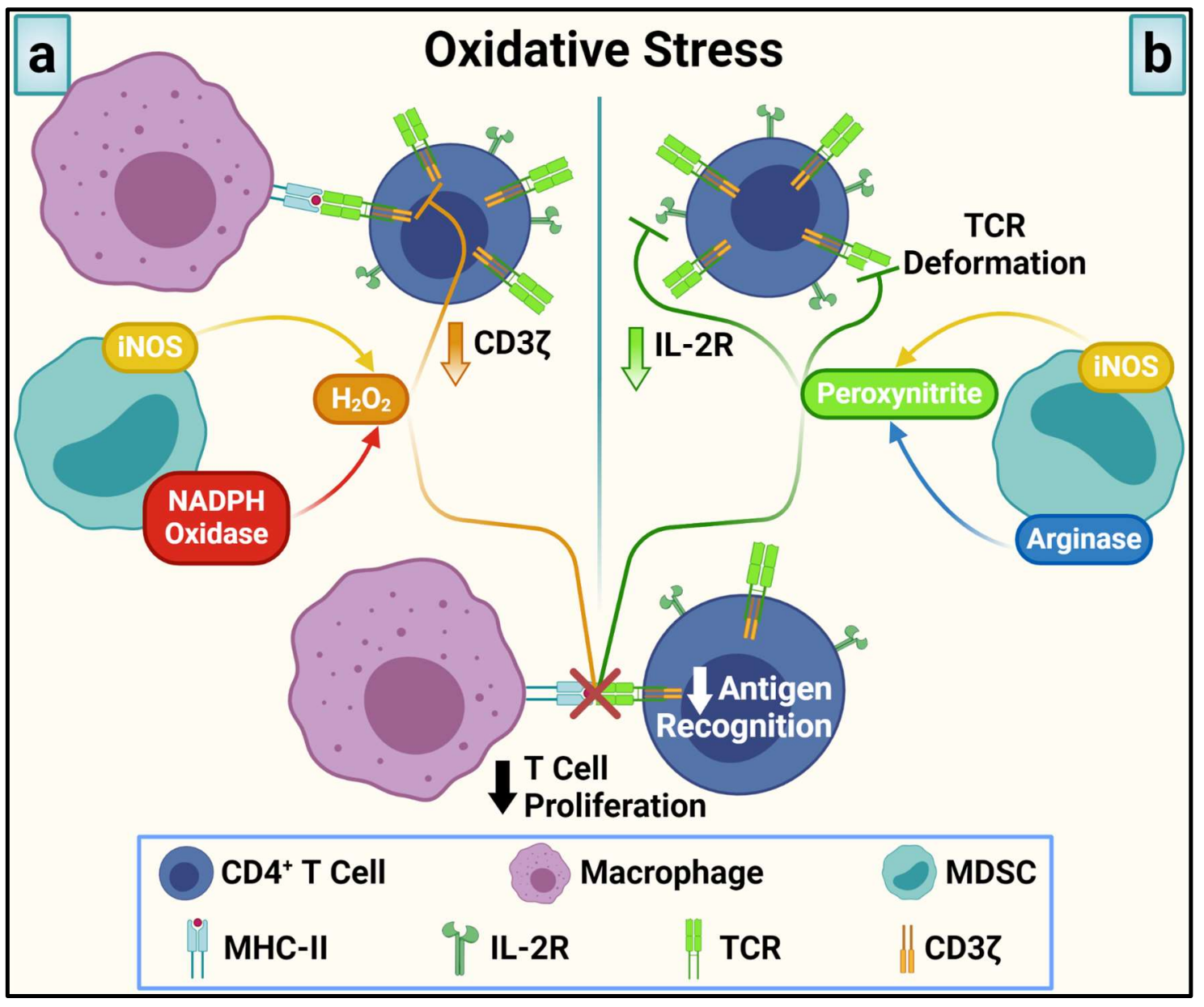

Figure 1.3: MDSCs use oxidative stress responses to prevent $T$ cell activation and subsequent proliferation in the tumor microenvironment. (a) NADPH oxidase and iNOS activity increases hydrogen peroxide levels in the microenvironment, prompting $T$ cells to downregulate $\mathrm{CD} 3 \zeta$ expression and ultimately disrupting their ability to respond to antigen. (b) Peroxynitrite deforms surface proteins such as T cell receptors (TCR) and IL-2R. In the absence of continued IL-2 signaling and antigen recognition. T cell proliferation grinds to a halt. MDSC: myeloid-derived suppressor cell. 


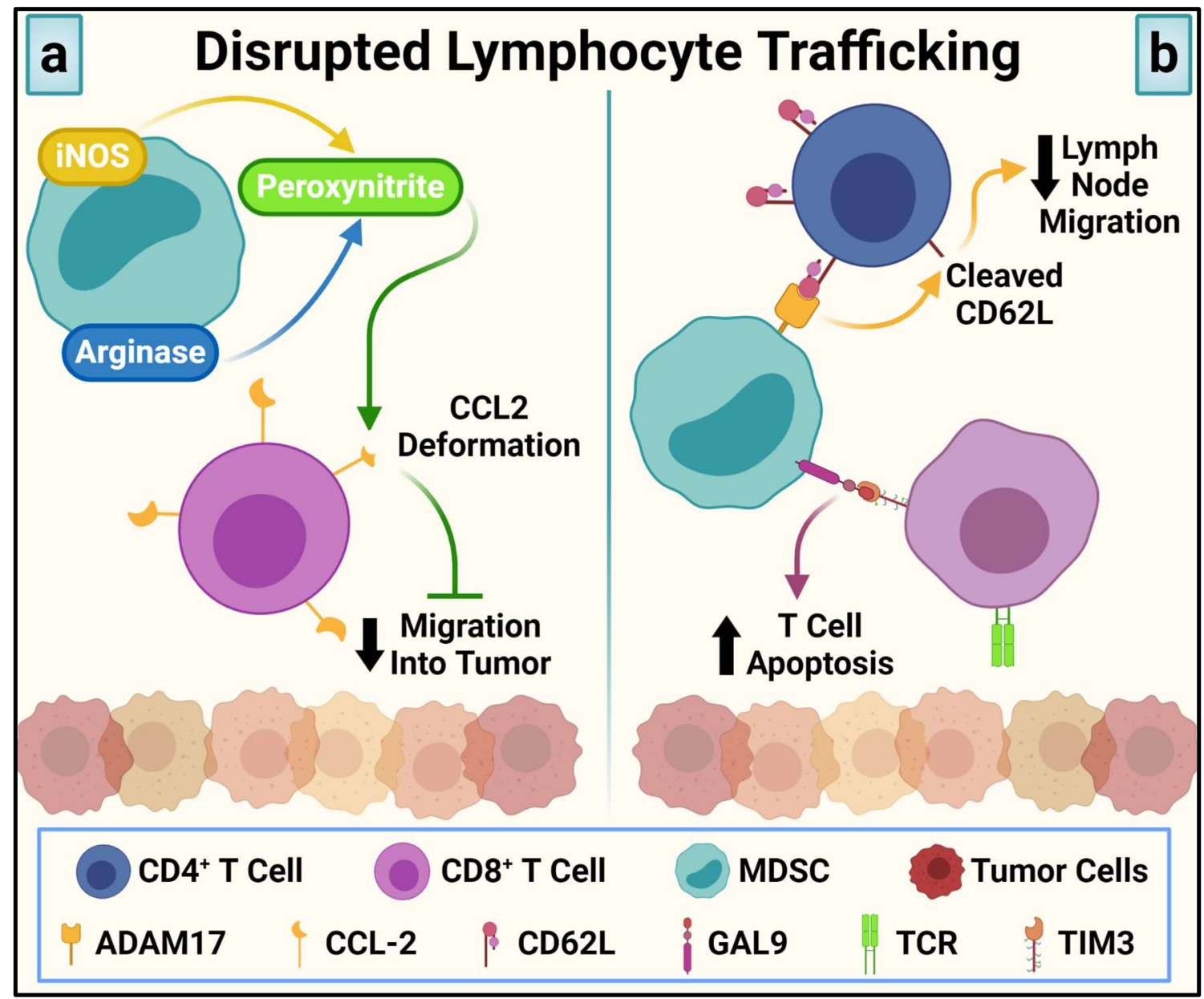

Figure 1.4: MDSCs disrupt lymphocyte trafficking into and out of the tumor microenvironment. (a) MDSC-mediated nitrosylation of CCL2 interrupts the signaling pathway that recruits $\mathrm{CD} 8^{+}$cytotoxic $\mathrm{T}$ cells into tumor tissue. (b) MDSCs interact directly with $\mathrm{T}$ cells to prevent their migration into the lymph nodes by disrupting CD62L expression or promoting cell death. MDSC: Myeloid-derived suppressor cell. TCR: T cell receptor. 


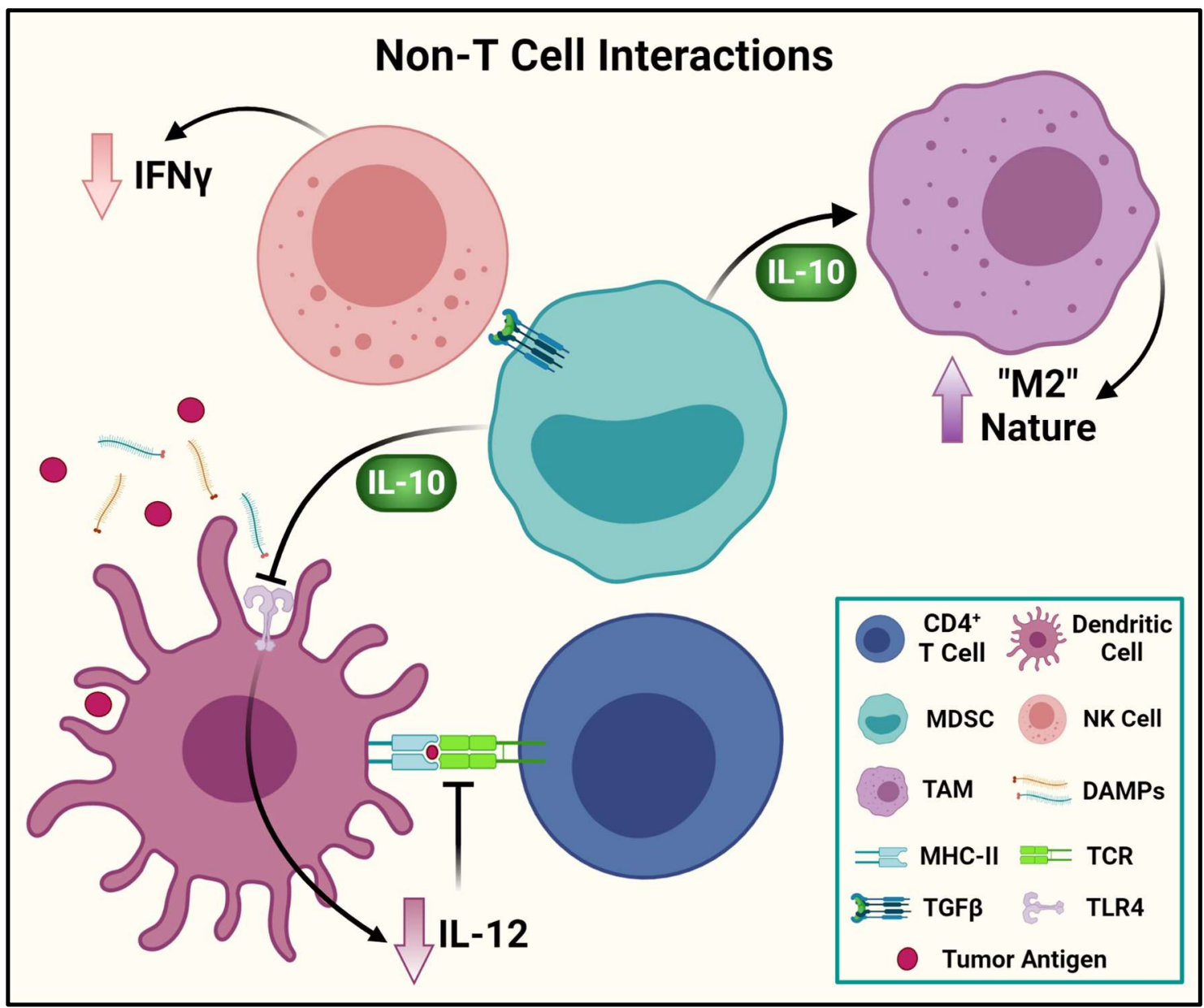

Figure 1.5: Immunomodulatory effects of MDSCs on innate immune cells. In addition to T cells, MDSCs can manipulate the inflammatory responses of other cells in the tumor microenvironment using contact-dependent and independent mechanisms. MDSC: myeloid-derived suppressor cell. TAM: tumor-associated macrophage. DAMPs: damage-associated molecular pattern. TCR: T cell receptor. 


\section{MDSC Ontogeny}

Since their discovery the possible origins of MDSCs has been a subject of speculation. The prevailing hypothesis among MDSC biologists today suggests that generation of MDSCs in pathology occurs in two steps. First there is an expansion of the immature myeloid cell (IMC) population, subsequently followed by an "activation" signal leading to their acquisition of suppressive characteristics. This model is based upon the emergency and extramedullary myelopoiesis which is a result of the non-resolving or chronic inflammation induced by conditions such as cancer, sepsis, or autoimmunity [93, 119]. The prolonged inflammatory pressures at these sites promotes the migration of IMCs from the bone marrow to replace exhausted or dead myeloid populations at the origin of the insult. Additional expansion of this IMC population can occur in the spleen in response to secondary tumor-derived signals. It's this secondary signal, usually in the form of proinflammatory cytokines (IFNY), Th2-associated cytokines (IL-4 or IL-13), or TLR ligands (DAMPs), that causes the cells to develop suppressive functional abilities [99].

An alternative hypothesis concerns the nature of the secondary, "activation" signal. In this model abnormally prolonged inflammation still induces emergency and extramedullary myelopoiesis, mobilizing and expanding IMCs. In this case however, the secondary signal arrests any further maturation of these cells [120123]. Here it is assumed that the suppressive activities of MDSCs are actually an inherent quality possessed by all IMCs which differentiate into macrophages, DCs, and neutrophils so quickly under steady-state conditions that these abilities are of little consequence. 
The exact "expansion" and "activation" signals that lead to the accumulation of MDSCs vary greatly depending upon the condition and even differ among tumor models. These cells are highly susceptible to signals in their microenvironment and will attune their gene expression and function in response [101]. In practice this means that MDSCs isolated from two different kinds of tumors may have starkly different transcriptional profiles, making it difficult to accurately identify a signature "MDSC-inducing" signaling pathway. There are some candidate pathways and transcription factors however that have been linked to their myeloid suppressor phenotype (Figure 1.6). STAT3 activation is found almost universally within any MDSC population being characterized. IL-1 $\beta$, IL-6, IL-10, GM-CSF, and G-CSF produced in the TME have all been associated with inducing STAT3-mediated transcription of Nox2 and Arg1, both of which regulate ROS-based immune suppression or the expansion of MDSCs at the tumor site [107]. IL-1 $1 \beta$ and IFNYmediated signaling through STAT1 in addition to IL-4 and IL-13-mediated activation of STAT6 also promote MDSC immunosuppressive activity by upregulating Nos2, Arg1, and Nox2 expression. STAT-dependent gene transcription also contributes to immature myeloid cell migration from the bone marrow, MDSC differentiation, and their accumulation at the tissue site via $B c / 2 / 1$ and S100a8/9 expression induced by GM-CSF signaling through STAT3 and STAT5 [124].

Common among many MDSC researchers who subscribe to the classic "two-signal" model, is a steadfast belief that MDSCs arise from an abnormal response to pathologically prolonged or non-resolving inflammation. In other 
words, there is an inherent defect that has inappropriately sustained a proinflammatory response and it's only under these conditions that MDSCs develop $[97,100]$. Central to this is the assumption that MDSCs are fundamentally distinct from other immune cells, such as $\mathrm{T}_{\text {Reg }}$ and "M2" macrophages, that accumulate during the resolution phase of a normal immune response to promote wound healing and prevent further inflammation-derived tissue damage.

There is some evidence that this way of thinking about MDSCs may not be quite as accurate as it was once considered to be. For instance, MDSCs have recently been shown to be essential for the maintenance of maternal-fetal tolerance. If MDSCs were generated by a defective immune response, then they would not be so closely associated with normal, successful pregnancies [90, 97]. MDSC recruitment during sepsis may provide additional evidence for this. These cells may impair bacteria-clearing immune responses at later time points, but their presence in early stages of septicemia prevents the detrimental tissue damage caused by the extreme inflammatory responses this condition elicits $[93,125]$. With this in mind, some researchers have begun reexamining the nature of this original "two-signal" model with a few even rethinking what kinds of cells should or should not be considered MDSCs [93]. As Millrud, Bergenfelz [93] stated in their 2016 review On the origin of myeloid-derived suppressor cells, "An important issue concerning MDSC generation to remember is that tumors do not invent new biology, they highjack existing mechanisms."

Along these lines, recent studies on the nature of neutrophils have shown that, once activated, these cells can live much longer than we originally thought 
and can develop either a pro- or anti-inflammatory phenotype based upon their surroundings. Initially these cells were separated from PMN-MDSCs using a density gradient since they were significantly more granular [126]. New evidence indicates however, that degranulated neutrophils are less dense and settle at a similar level as PMN-MDSCs following density centrifugation. This has led some researchers to hypothesize that a portion of the heterogeneous subset we classify as PMN-MDSCs, may actually be degranulated, immunomodulatory tumorassociated neutrophils [100].

As for the generation of M-MDSCs I prefer the model proposed by Millrud, et al. (2016) in the review quoted above. Like the classic hypothesis, theirs also consists of two signals, however instead of the "expansion" and "activation" of IMCs, they propose a model whereby monocytes are "recruited" and "reprogrammed". Damage or pathogen associated molecules trigger toll-like receptors on the surface of monocytes, recruiting these cells out of circulation. Once in the tissue or tumor site, these cells continue to encounter TLR agonists in addition to immunomodulatory signals such as IL-10. This combination of pro- and anti-inflammatory stimuli "reprograms" the monocyte, causing it to acquire immune suppressive functions [93]. The "recruit and reprogram" model is particularly appealing as it places M-MDSCs on a similar level as any other cell type that gets reprogrammed by tumor-derived signals. In either case, it is becoming increasingly clear with each new non-cancer-centric MDSC study that it is time to reassess not only our model of how MDSCs are generated, but also our one dimensional, pathology-oriented idea of these complicated cells. 


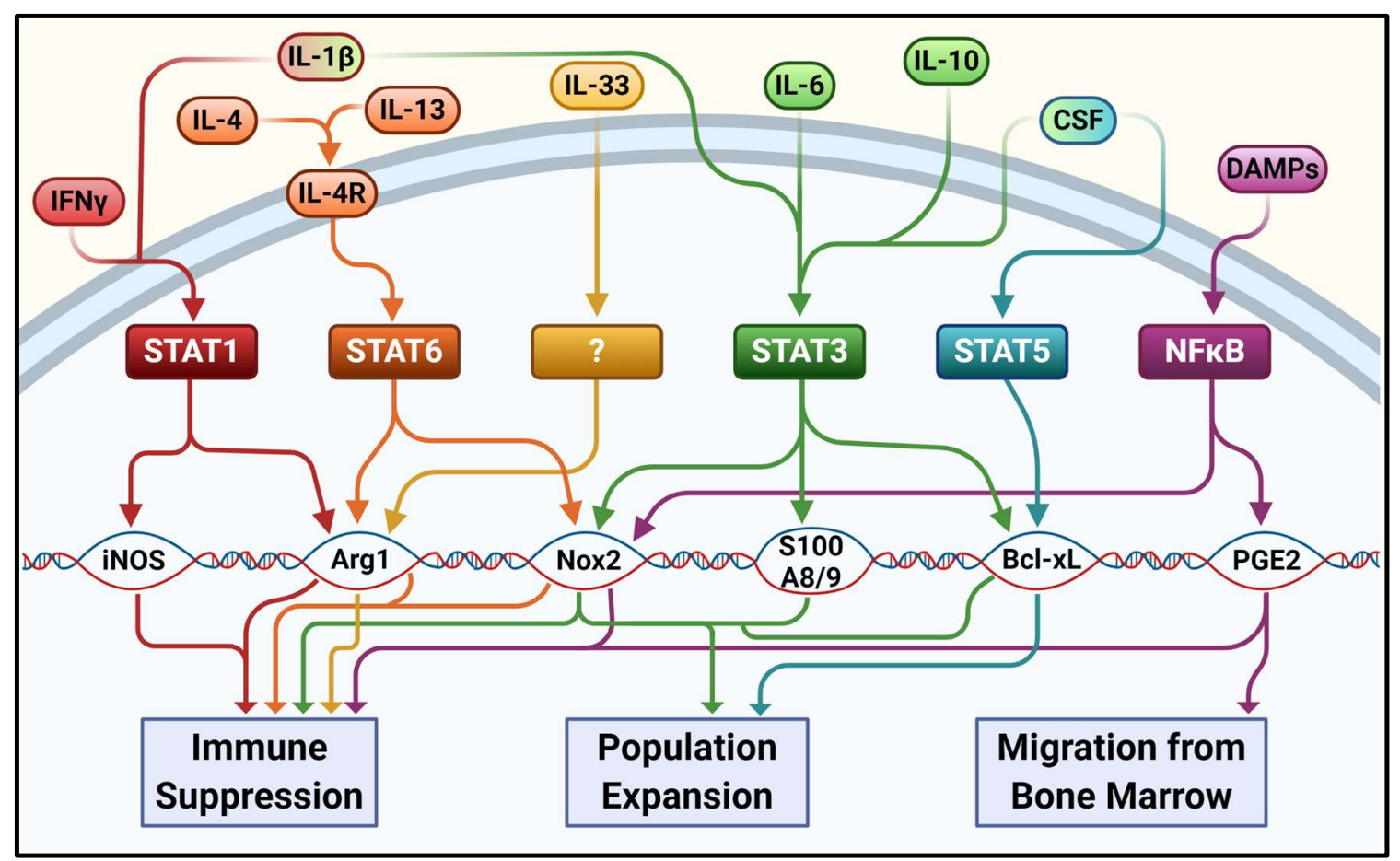

Figure 1.6: Candidate signaling cascades contributing to MDSC accumulation and suppressive phenotype. The plasticity of MDSCs makes it difficult to precisely define a single "MDSC-inducing" signaling profile, there are some however that have been linked with various aspects of MDSC biology. DAMPs: danger-associated molecular patterns. 


\section{MDSCs in Healthy Tissues}

Our understanding of MDSC biology has greatly expanded since they were first described almost 20 years ago. Despite the growing list of conditions in which MDSCs play a beneficial role, the majority of studies on their biology is still done within the realm of cancer. Furthermore, the assumption that these cells only arise from pathologically induced abnormal maturation still permeates the field. CD11 $\mathrm{b}^{+}$ $\mathrm{Gr}^{+}$cells found in healthy tissues are often dismissively classified as immature myeloid cells without further functional studies since it's assumed that the immunosuppressive activity of those cells only arises as a consequence of inappropriately prolonged emergency myelopoiesis or chronic inflammation.

The endurance of this belief may be strongly linked to their original discovery in the tumor microenvironment and the profound role they play in priming it for optimum immune evasion. If these cells had been discovered under different conditions, investigations may not have been so narrowly focused. For instance, the immunosuppressive nature of tumor-associated macrophages is considered a pathological consequence of the tumor microenvironment on these cells, but macrophages in and of themselves are not associated solely with disease. Even immunosuppressive "M2-like" macrophages are seen as physiologically normal in steady-state conditions. Testing the functional activity of macrophages isolated from healthy tissues is important and, for the most part, seen as a necessary step in characterizing a novel population.

The explosion of $\mathrm{CD} 11 \mathrm{~b}^{+} \mathrm{Gr} 1^{+}$cells under chronic inflammatory pressures should also not be used to justify our disregard for the effector functions of these cells in steady state. Knowing that $\mathrm{T}$ cells clonally expand following antigen 
recognition does not prevent $\mathrm{CD} 3^{+} \mathrm{CD} 4^{+}$cells from being recognized as bona fide T cells when they're isolated from healthy tissues.

Molecular signals within the surrounding tissue have a profound impact on macrophage and T cell differentiation and functional phenotype. It's thought that a similar dynamic occurs with MDSCs within the tumor microenvironment, as MDSCs isolated from different types of tumors often exhibit different patterns of gene expression and use different mechanisms to suppress inflammatory responses [102]. Furthermore, several studies over the last few years have revealed more and more non-tumor associated instances that give rise to MDSCs [90] [90-92]. In autoimmune diseases such as rheumatoid arthritis, MDSCs suppress inflammatory $\mathrm{T}$ cell responses and drive $\mathrm{T}_{\text {Reg }}$ induction via their production of IL-10 [127]. MDSCs have also been identified outside the realm of pathology altogether. Although the situations described thus far represent physiologically normal, perturbations of tissue homeostasis such as becoming pregnant or being recently born. In each case MDSCs play a protective role against fetus-directed and overactive immune responses respectively $[90,128]$. To me, this all indicates that the suppressive MDSC phenotype develops more from the cues an immature myeloid cell encounters within a particular microenvironment than as a consequence of the pathology itself. Taken one step further, this implies that any tissue containing the appropriate combination of molecular signals could give rise to its own population of MDSCs.

As the list of MDSC-inducing conditions grows and begins to include physiologically normal situations like pregnancy [90], MDSC biologists need to 
reexamine their perceptions of these cells. Perhaps even consider the possibility that they are alternatively activated monocytes and neutrophils involved in mediating tissue homeostasis and repair, just like their alternatively activated macrophage counterparts.

\section{The MDSC-inducing Potential of Healthy Adipose Tissue}

Both leptin knockout and high-fat diet-fed mice develop chronic, low-grade adipose tissue inflammation, which leads to the accumulations of MDSCs [129]. Similar to what is seen in autoimmune-induced inflammation these cells play a protective role in the tissue by offsetting the metabolic dysfunction associated with obesity $[129,130]$. Intriguingly the studies that first identified these cells in inflamed adipose also describe a population of $\mathrm{CD} 11 \mathrm{~b}^{+} \mathrm{Gr} 1^{+}$cells in the adipose of their normal diet-fed, negative control mice. Unfortunately, the immunomodulatory functions of these cells were never tested, as it was assumed that cells with these surface markers did not develop immunosuppressive capabilities in the absence of pathological pressures. However, the microenvironments of tumors and healthy adipose tissue share many common elements as they both have a propensity for employing a network of cellular and molecular players to promote and sustain robust anti-inflammatory conditions. Surprisingly, many of the shared elements of this network are involved in the recruitment of MDSCs by tumors. Therefore, if we assume that an MDSC acquires its immunomodulatory nature as a consequence of signals from its surroundings and not pathology itself, then it's likely that the $\mathrm{CD} 11 \mathrm{~b}^{+} \mathrm{Gr} 1^{+}$cells in the adipose of their negative controls would have inhibited 
activated $\mathrm{T}$ cell responses. Table 1.2 highlights some of the key similarities between tumors and healthy adipose tissue.

On a macro level, both tumors and adipose are dynamic tissues where both pro- and anti-inflammatory stimuli are found. The coexistence of these diametrically opposed signaling molecules are essential elements in the "Recruit and Reprogram" model of MDSC generation [93]. From a cell network point of view, tumor-associated macrophages (TAMs) are one of the driving forces behind MDSC recruitment and retention at the tumor site. TAMs provide IL-10 which reprograms recruited monocytes into suppressive MDSCs. Additionally, IL-10 produced by MDSCs promotes the "M2" phenotype of TAMs, establishing a mutually beneficial feedback loop. This allows for more MDSCs to accumulate and strengthen the immunosuppressive nature of TAMs. In healthy adipose tissue, the similarly "M2" skewed tissue-resident macrophage (ATM) population produces IL$10[20,131,132]$ which is a necessary component of the anti-inflammatory AT environment.

T cells play a critical role in creating the optimum niche for MDSCs in the tumor microenvironment. For instance, IFNY and TNFa produced by tumorinfiltrating effector $\mathrm{T}$ cells provide the chronic myelopoiesis and the eventual recruitment of immature myeloid cells (IMCs) to the tumor site [133]. Meanwhile, induced regulatory $\mathrm{T}$ cells prime the microenvironment with IL-10 and TGF $\beta$ contributing to the pool of anti-inflammatory cytokines that can reprogram IMCs into MDSCs $[134,135]$. Both effector memory and regulatory $T$ cells are present in healthy adipose tissue. 
Some of the other players in the adipose immune cell network such as ILC2s and NKT cells are also involved in establishing the immunosuppressive conditions in the tumor microenvironment. In both tumors and adipose these cells promote "M2" polarization of the resident macrophage populations via IL-13 production [136-138]. This cytokine can also contribute to the suppressive phenotype of MDSCs. Collectively, these similarities with the tumor microenvironment make healthy adipose tissue a likely niche for MDSCs to accumulate. 
Table 1.2: Similarities Between the Tumor \& Adipose Microenvironments.

\begin{tabular}{|c|c|c|}
\hline Cell Type & $\begin{array}{c}\text { Tumor } \\
\text { Microenvironment }\end{array}$ & $\begin{array}{l}\text { Adipose Tissue } \\
\text { Microenvironment }\end{array}$ \\
\hline Macrophages & $\begin{array}{l}\text { IL-10 feedback loop } \\
\text { between } \mathrm{T}_{\text {reg } ~ \& ~ M D S C s} \\
\text { strengthen "M2" } \\
\text { phenotype } \\
\text { IL-10 promotes monocyte } \\
\text { reprogramming into } \\
\text { MDSCs }\end{array}$ & $\begin{array}{l}\text { IL-10 from Tre \& NKT } \\
\text { cells promotes "M2" } \\
\text { phenotype } \\
\text { IL-10 sustains } \\
\text { suppressive nature of } \\
\text { other AT immune cells }\end{array}$ \\
\hline $\begin{array}{c}\text { Regulatory } T \text { Cells } \\
\left(T_{\text {reg }}\right)\end{array}$ & $\begin{array}{l}\text { Produce IL-10 \& TGF } \beta \\
\text { which promote "M2" TAM } \\
\text { \& MDSC suppressive } \\
\text { phenotypes }\end{array}$ & $\begin{array}{l}\text { Population grows over } \\
\text { time } \\
\text { Produce IL-10 \& TGF } \beta \\
\text { which promote "M2" ATM } \\
\text { polarization \& suppress } \\
\mathrm{T}_{\text {EM }} \text { responses }\end{array}$ \\
\hline $\begin{array}{l}\text { Effector / Effector } \\
\text { Memory T Cells }\end{array}$ & $\begin{array}{l}\text { Produce IFNy \& TNFa } \\
\text { which recruit IMCs to } \\
\text { tumor site } \\
\text { IL-10 \& TGF } \beta \text { suppress } \\
\text { effector responses }\end{array}$ & $\begin{array}{l}\text { Primarily quiescent } \\
\text { memory phenotype } \\
\text { Mount rapid IFNy \& TNFa- } \\
\text { driven inflammatory } \\
\text { responses }\end{array}$ \\
\hline ILC2s & $\begin{array}{l}\text { IL-25 \& IL-13 induce Th2 } \\
\text { T cell responses which } \\
\text { are protumoral } \\
\text { Recruited by stromal cell } \\
\text { produced IL-33 }\end{array}$ & $\begin{array}{l}\text { Produce IL-13 \& IL-5 } \\
\text { which promote "M2" ATM } \\
\text { phenotype } \\
\text { Aide in eosinophil } \\
\text { recruitment via IL-5 } \\
\text { O } \\
\text { Recruited to AT by IL-33 }\end{array}$ \\
\hline NKT Cells & $\begin{array}{l}\text { Produce IL-13 that } \\
\text { promotes protumoral type } \\
2 \text { immune responses. } \\
\text { IL-10 \& IL-13 promote } \\
\text { "M2" TAM \& MDSC } \\
\text { suppressive phenotypes }\end{array}$ & $\begin{array}{l}\text { Produce IL-10, IL-12, and } \\
\text { IL-13 which contribute to } \\
\text { "M2" ATM phenotype } \\
\text { IL-12 contributes to } \\
\text { maintenance of } \mathrm{T}_{\text {reg }} \\
\text { population. }\end{array}$ \\
\hline
\end{tabular}




\section{CHAPTER 2: METHODS \& MATERIALS}

\section{Animals}

C57BL/6J mice were purchased from Jackson Laboratory [Bar Harbor, ME, USA] or bred in-house. Unless stated otherwise all reported findings were from 1026-week-old male mice. All animal studies were performed under protocols approved by the University of Louisville Institutional Animal Care and Use Committee and complied with the National Institutes of Health Guidelines for the Care and Use of Laboratory Animals.

\section{Stromal Vascular Fraction Isolation}

Perigonadal fat pads (epididymal or uterine horn) were harvested from 5-10 mice and collected in sterile $0.1 \%$ BSA solution. In a sterile petri dish, the pooled adipose tissue was manually homogenized with scissors. $1 \mathrm{~mL}$ of Digestion Buffer (2mg Collagenase [Worthington Biochemical Corporation; Lakewood, NJ, USA], 1mg DNase [Sigma-Aldrich; St. Louis, MO, USA], 1mL 0.1\% BSA) per milliliter adipose tissue was added to the homogenized tissue. The total solution (adipose + digestion buffer) was collected in a $50 \mathrm{~mL}$ conical vial containing a stir bar and digested at $37^{\circ} \mathrm{C}$ in an Envirogenie $(37.5 \mathrm{rpm})$ for 35 minutes. Following digestion, the contents of the tube were transferred to fresh $15 \mathrm{~mL}$ conical vials and centrifuged for 4 minutes at $550 \mathrm{xg}$. The supernatant was discarded; the cell pellet was resuspended in $0.1 \% \mathrm{BSA}$; and centrifuged once again for 3 minutes at $400 \mathrm{x}$ g. Cell were resuspended in $0.1 \%$ BSA and passed through a $20 \mu \mathrm{m}$ filter.

Some experiments required SVF cells to be isolated from individual mice, in order to fully appreciate the variability that exists in terms of adipose stromal cell 
make-up. For these isolations, the epididymal fat pads were collected from each animal in $3 \mathrm{~mL} 0.1 \% \mathrm{BSA}$ and kept at $4^{\circ} \mathrm{C}$ until they were processed. The tissue was then transferred to a fresh $5 \mathrm{~mL}$ tube where it was manually homogenized with scissors. One volume of Digestion Buffer was added, and the tubes were incubated at $37^{\circ} \mathrm{C}$ in a tube rotator for 35 minutes. When processing multiple samples at once, homogenization and incubation was staggered to ensure even digestion times for each sample. Following digestion, cold $0.1 \%$ BSA was added to each tube. Samples were centrifuged at $550 \times \mathrm{g}$ for 4 minutes. Supernatants were discarded, and the pellet was transferred to a new $5 \mathrm{~mL}$ tube. After resuspension, the cells were centrifuged once more at $400 \mathrm{xg}$ for 3 minutes. Fresh $0.1 \%$ BSA was added to the washed cells which were then filtered through a $20 \mu \mathrm{m}$ filter cup [pluriSelect Life Science; Leipzig, Germany]. To ensure maximum cell yield, the tube was rinsed twice and the filter once with $5 \mathrm{~mL} 0.1 \%$ BSA. This individual cell isolation protocol produced cell yields and population distributions that were comparable to our pooled SVF isolation protocol.

\section{Flow Cytometry Staining for Analysis}

Adipose stromal cells and splenocytes were incubated in Fc block for 10 minutes in the dark, at room temperature. Cells were stained with appropriate monoclonal antibodies for 20-30 minutes at room temperature. Table 2.1 contains the antibodies included in each staining panel used in this study. Intracellular staining for Foxp3 and Ki-67 was performed using Foxp3/Transcription Factor Staining Kits [eBioscience; San Diego, CA, USA] according to the manufacturer's instructions. Data was collected on an LSR II cytometer [BD Biosciences; Franklin 
Lakes, NJ, USA] and analyzed with FlowJo software [Tree Star; Ashland, OR, USA]. Compensation beads [BD Biosciences] were used for data collection, but compensation was manually adjusted during analysis to account for the autofluorescent nature of adipose stromal cells. 
Table 2.1: List of Antibodies Used in Flow Cytometry Panels.

\begin{tabular}{|c|c|c|c|c|}
\hline Target & Fluorophore & Clone & Vendor & Catalog \# \\
\hline CD3 & APC & $17 A 2$ & ThermoFisher & $17-0032-80$ \\
\hline CD4 & BV421 & RM4-5 & BioLegend & 100563 \\
\hline CD4 & PE/Cy5 & GK1.5 & VWR & $100409-\mathrm{BL}$ \\
\hline CD4 & APC/Fire 750 & GK1.5 & VWR & $100459-\mathrm{BL}$ \\
\hline CD8 & BV510 & $53-6.7$ & VWR & $100751-\mathrm{BL}$ \\
\hline CD8 & BV605 & $53-6.7$ & VWR & $100743-\mathrm{BL}$ \\
\hline CD11b & APC & M1/70.15.11.5 & Miltenyi & $130-113-793$ \\
\hline CD11b & PE & M1/70.15.11.5 & Miltenyi & $130-113-797$ \\
\hline CD11c & FITC & N418 & VWR & $117305-\mathrm{BL}$ \\
\hline CD44 & APC/Cy7 & IM7 & VWR & $103027-\mathrm{BL}$ \\
\hline CD45 & APC/Fire 750 & $30-F 11$ & VWR & $103153-\mathrm{BL}$ \\
\hline CD62L & FITC & MEL14-H2.100 & Miltenyi & $130-102-465$ \\
\hline CD69 & BV605 & H1.2F3 & VWR & $104529-B L$ \\
\hline CD301 & Alexa Fluor 647 & ER-MP23 & Bio-Rad & MCA2392a647T \\
\hline F4/80 & PerCP/Cy5.5 & BM8 & VWR & $123127-B L$ \\
\hline FcR Block & - & $2.4 G 2$ & BD & 553142 \\
\hline Foxp3 & PE & FJK-61s & Fisher & $50-112-9518$ \\
\hline Gr1 & - & RB6-8C5 & BioXCell & BE0075 \\
\hline IL33R (ST2) & APC & RMST2-2 & Fisher & $50-112-8785$ \\
\hline Ki67 & PE & $16 A 8$ & BioLegend & 652403 \\
\hline Ly6C & BV421 & HK1.4 & VWR & $128031-B L$ \\
\hline Ly6C & FITC & HK1.4 & VWR & $128006-B L$ \\
\hline Ly6G & BV605 & 1 A8 & Fisher & BDB563005 \\
\hline Ly6G & PE/Cy7 & $1 \mathrm{~A} 8$ & VWR & $127617-B L$ \\
\hline 3HC-II (IA/IE) & PE/Cy7 & M5/114.15.2 & VWR & $107629-B L$ \\
\hline itrotyrosine & - & $7 A 12 A F 6$ & Abcam & ab110282 \\
\hline
\end{tabular}




\section{Cell Sorting}

For sorting adipose-derived MDSCs (aMDSCs), total adipose stromal cells were stained with CD11b APC [Miltenyi Biotec; San Diego, CA, USA], Ly6C FITC [VWR; Radnor, PA, USA], and Ly6G PE-Cy7 [VWR]. CD11b ${ }^{\mathrm{Hi}} \mathrm{Ly}^{6 \mathrm{C}^{\mathrm{Hi}}}$ Ly6GSSCLow cells were then sorted by the University of Louisville Microbiology and Immunology Flow Cytometry Core Facility on a FACS Aria III [BD Biosciences] (Figure 2.1 a). We found that stresses inherent to the sorting process negatively impacted the suppressive phenotype of FACS-isolated MDSCs. However, if the cells were allowed to recover for $4-5$ hours at $4^{\circ} \mathrm{C}$ they regained their immunomodulatory functionality [139, 140] (Figure 2.2).

For sorting adipose tissue T cells and macrophages, total adipose stromal cells were stained with CD11b PE [Miltenyi Biotec], Ly6C FITC [VWR], CD4 APCCy7 [VWR], MHC-II PE-Cy7 [VWR], and CD301 AlexaFluor647 [Bio-Rad;

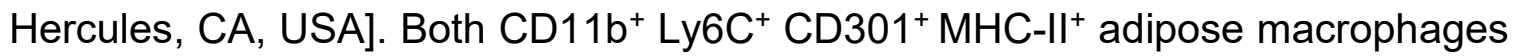
(Figure 2.1 a) and CD11 b- CD4+ adipose T cells were collected (Figure 2.1 b). 

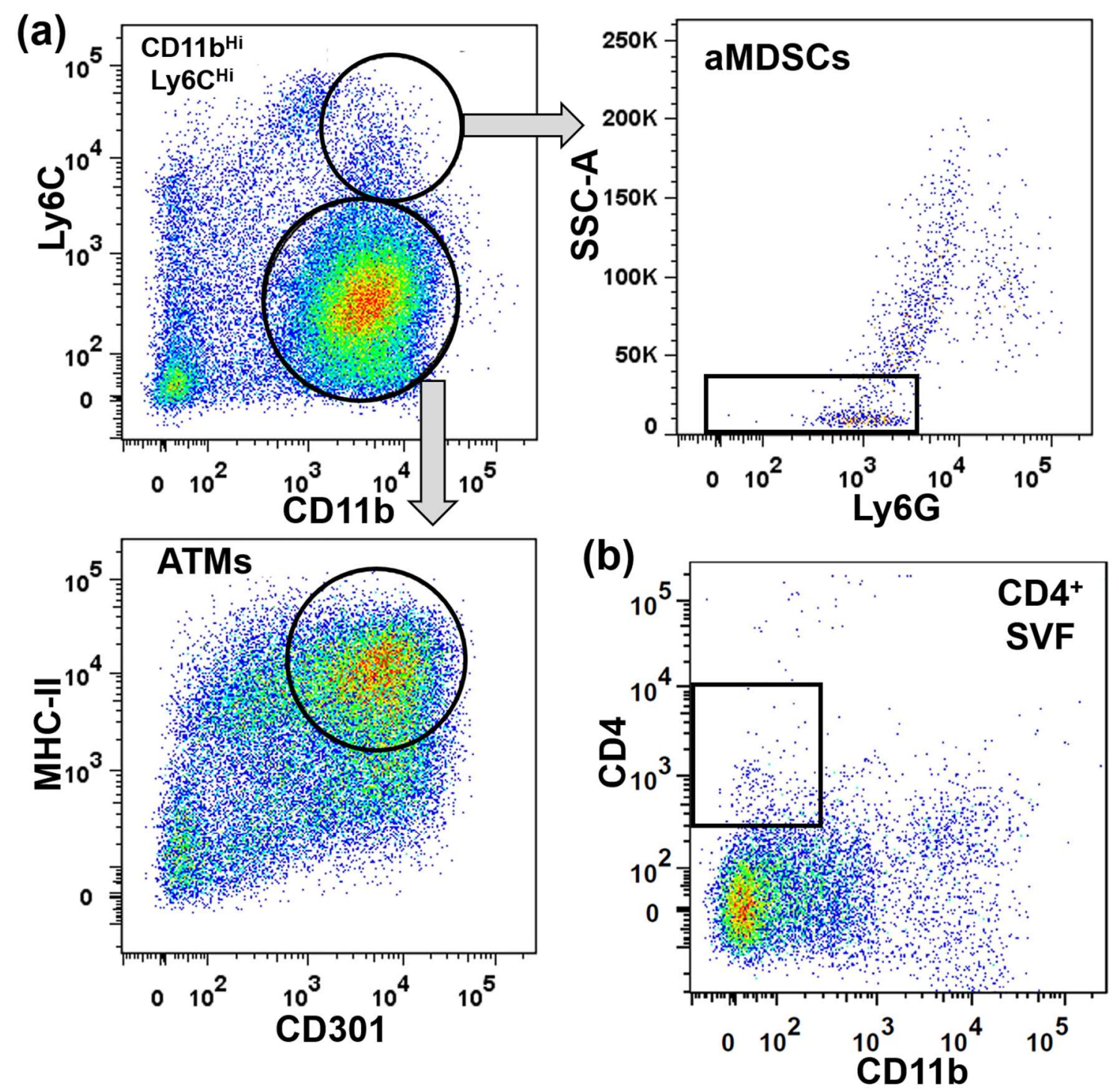

Figure 2.1: Cell sorting strategies for FACS isolation of SVF subpopulations. (a) MDSC \& adipose macrophage sort strategy (b) Adipose tissue T cell sort strategy. 
(a)

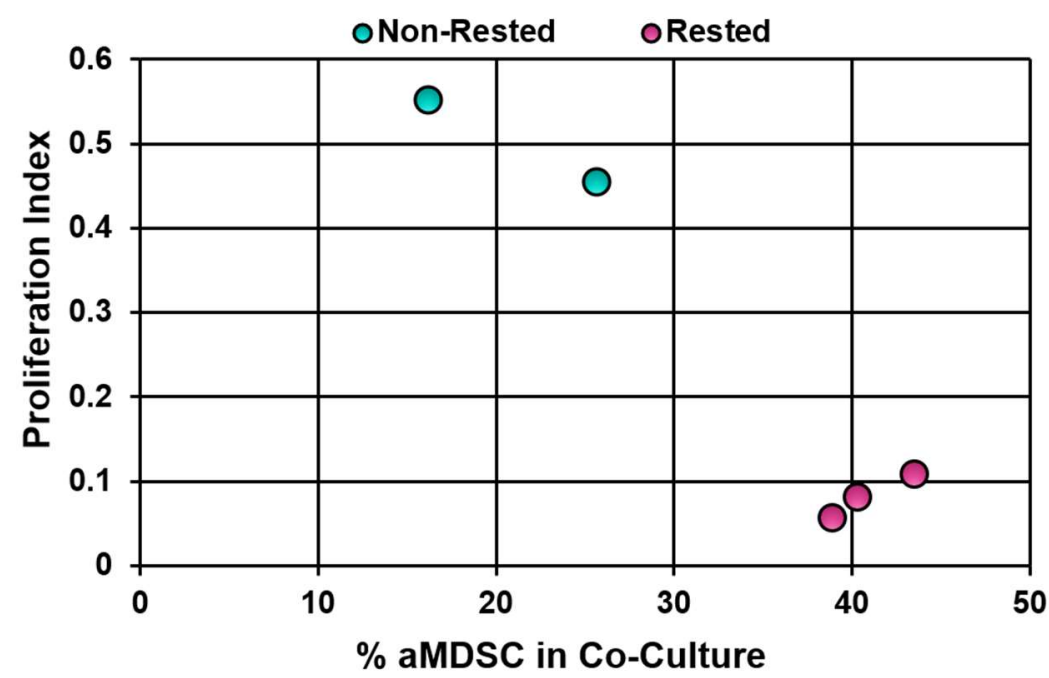

(b)

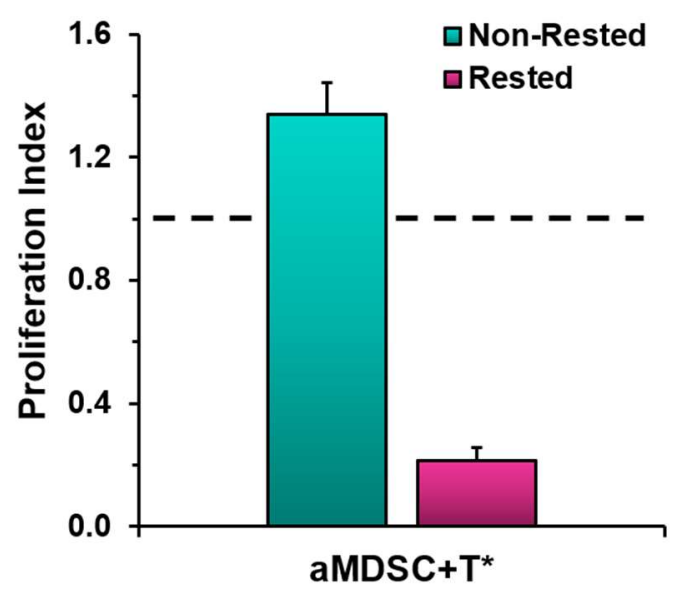

Figure 2.2: FACS isolated aMDSCs require a brief rest period before use in proliferation assays. Sorted aMDSCs were either immediately resuspended in RPMI and plated (non-rested) or left in sort buffer at $4^{\circ} \mathrm{C}$ for 3 hours before being resuspended in media and plated (rested). aMDSCs were co-cultured with aCD3/aCD28 activated MACS-enriched T cells. After five days $T$ cell proliferation was assessed by measuring Abs at $450 \mathrm{~nm}$. Abs values within each experiment were normalized to create a PI relative to the mean Abs of aCD3/aCD28stimulated MACS-enriched T cells. Finally, each co-culture was then analyzed via flow cytometry to determine what proportion of the remaining live cells was made up of aMDSCs. (a) The frequency of remaining aMDSCs in each co-culture versus activated $\mathrm{T}$ cell proliferation (Proliferation Index). Each circle represents an individual well. (b) $\alpha \mathrm{CD} 3 / \alpha C D 28$-stimulated $\mathrm{T}$ cell proliferation following five-day co-culture with non-rested (Blue) or rested (Pink) aMDSCs. 


\section{T Cell Proliferation/Suppression Assays}

Adipose stromal cells were processed and isolated as described from 10, 16 - 20-week old, male mice. Cells were then stained and sorted as described above. While the sorted cells were resting, spleens from 2-3 mice were manually disrupted and passed through a $100 \mu \mathrm{m}$ cell strainer. The single cell suspension was washed twice with sterile HBSS and filtered a final time through a $70 \mu \mathrm{m}$ cell strainer. Total splenocytes were counted, then enriched for T cells using a negative selection magnetic bead separation kit [Miltenyi Biotec] according to the manufacturer's instructions. MACS-enriched T cells and sorted adipose-derived CD11 ${ }^{H i}$ Ly6C $^{H i}$ Ly6G- SSC ${ }^{\text {Low }}$ cells were resuspended in media [RPMI 1640 supplemented with 10\% FBS, 2-Mercaptoethanol, Pen/Strep, Fungizone, and EDTA] and plated at a 1:1 ratio in a 96-well culture plate. The final culture volume in each well was $100 \mathrm{uL}$. Some wells were coated with $1 \mathrm{ug} / \mathrm{mL} \alpha \mathrm{CD} 3$ and $3 \mathrm{ug} / \mathrm{mL}$ aCD28 to activate the T cells. Each culture condition was performed in triplicate.

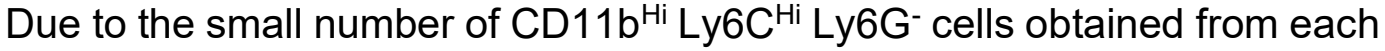
sort, the use of traditional flow cytometry or radiation-based proliferation assays was not feasible, as the low cell numbers made it difficult to reliably obtain repeatable results with these standard methods (See detailed explanation under

Chapter 3: Experimental Challenges \& Model Development). As an alternative, T cell proliferation was assessed, after 5 days, using a colorimetric MTT assay, according to the manufacturer's instructions [TetraZ Cell Proliferation Kit; BioLegend; San Diego, CA, USA] (Figure 2.3). Suppression of T cell proliferation was determined by establishing a "Proliferation Index" (PI) where the absorbance value obtained from each co-cultured well was divided by the mean absorbance of 
activated T cells or splenocytes alone. Mean PI values were calculated for each co-culture condition. Values greater than 1 indicated an increase in $\mathrm{T}$ cell proliferation, while $\mathrm{PI}$ values significantly less than 1 indicated that $\mathrm{T}$ cell proliferation had been suppressed. To further confirm that the differences in absorbance values between co-culture conditions were due to differences in $\mathrm{T}$ cell proliferation and not the result of variations in metabolic activity, we stained the cells with CD4 and CD8 following the final MTT measurement, then determined the absolute number of T cells in each well using flow cytometry using Accucount Beads [Spherotech; Lake Forest, IL, USA]. 


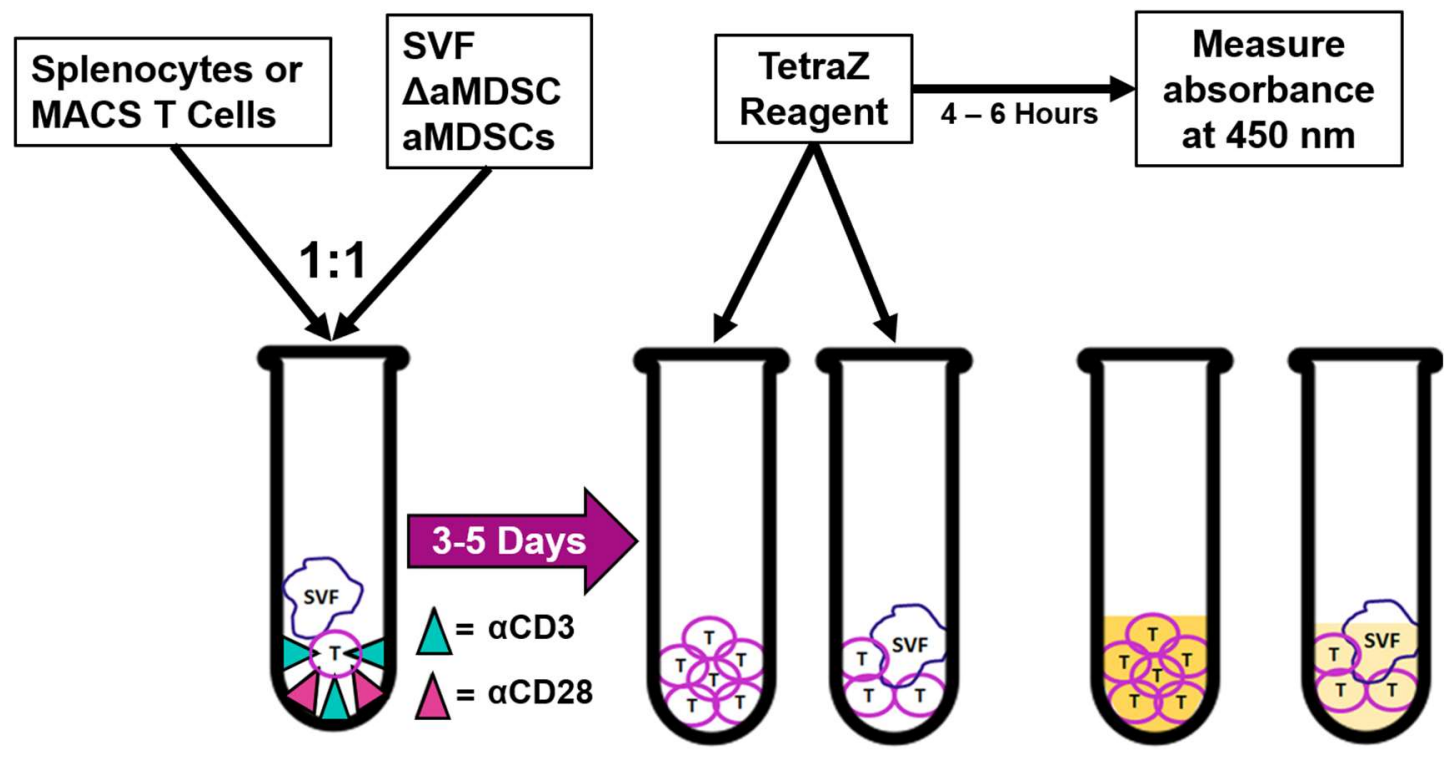

Figure 2.3: Schematic of TetraZ-based MTT assay workflow. Naïve splenocytes or MACS-enriched T cells are cultured by themselves, activated with plate-bound $\alpha C D 3 / \alpha C D 28$, or activated and co-cultured at a 1:1 ratio with either Full SVF, aMDSC-depleted SVF ( $\triangle$ aMDSCs), or sorted aMDSCs. After $3-5$ days, $10 \mu \mathrm{L}$ of TetraZ reagent is added to each well and returned to the incubator for $4-$ 6 hours. After incubation, absorbance is measured at $450 \mathrm{~nm}$. 


\section{RNA Isolation, RT-PCR \& qPCR}

RNA for this study was isolated with the RNeasy Mini or Micro Kits [Qiagen; Germantown, MD, USA] depending on the size of the sample. Prior to RNA isolation, cells and tissue lysates were homogenized with Qiashredder columns [Qiagen]. Lysates from whole adipose tissue were collected via Qiazol [Qiagen] and chloroform extraction. They were homogenized with the Qiashredder columns before RNA purification with the RNeasy Mini Kit [Qiagen]. cDNA synthesis was performed using SuperScript IV VILO with ezDNase [Thermo Fisher Scientific; Waltham, MA, USA]. SYBR Select Master Mix [Thermo Fisher Scientific] was used to perform qPCR on a StepOne Plus thermocycler [Applied Biosystems; Foster City, CA, USA]. In some cases, amplified DNA products were visualized by agarose gel separation. IL-2 amplification was performed with the Mm_II2_1_SG QuantiTect Primer Assay kit [Qiagen] while all other mouse primers were designed and purchased through IDT [Integrated DNA Technologies; Coralville, lowa, USA]. Primer sequences are listed in Table 2.2. 
Table 2.2: List of qRT-PCR primers.

\begin{tabular}{|c|c|c|}
\hline Target Gene & Forward $\left(5^{\prime} \rightarrow 3^{\prime}\right)$ & Reverse $\left(5^{\prime} \rightarrow 3^{\prime}\right)$ \\
\hline Arg1 & CTCCAAGCCAAAGTCCTTAGAG & AGGAGCTGTCATTAGGGACATC \\
\hline Ifng & ATCTGGAGGAACTGGCAAAA & TTCAAGACTTCAAAGAGTCTGAGG \\
\hline 1110 & AATAAGCTCCAAGACCAAGG & CAGACTCAATACACACTG \\
\hline $1 / 33$ & ATGGGAAGAAGCTGATGGTG & CCGAGGACTTTTTGTGAAGG \\
\hline Pparg & GAAGCGGTGAACCACTGATATT & GAGAGGTCCACAGAGCTGATT \\
\hline Tbp & CTACCGTGAATCTTGGCTGTAA & GTTGTCCGTGGCTCTCTTATT \\
\hline Tgfb1 & GGACTCTCCACCTGCAAGAC & GACTGGCGAGCCTTAGTTTG \\
\hline Tnfa & GAGAAAGTCAACCTCCTCTCTG & GAAGACTCCTCCCAGGTATATG \\
\hline
\end{tabular}




\section{In Vivo MDSC Depletion}

16-week-old male mice were injected subcutaneously with $15 \mu \mathrm{g} \mathrm{g}^{-1}$ of purified Gr-1 ( $a \mathrm{Gr} 1$ ) antibody [Clone: RB6-8C5] or Rat IgG2b isotype control [Clone: LTF-2] [BioXCell; West Lebanon, NH, USA], every 3-4 days throughout the duration of the experiment. Animals were weighed at each injection to monitor for any negative metabolic effects of the treatment. Both epididymal fat pads were collected at the end of the experiment (no more than two days after the final injection). Spleens were collected from each mouse to verify successfully MDSC depletion and check for any other systemic effects induced by the treatment.

\section{ELISAs}

T cell proliferation assays were set up as described above. After two days, $50-100 \mu \mathrm{L}$ of supernatant was collected from each well and stored at $-80^{\circ} \mathrm{C}$ in a separate 96-well plate. A matching volume of media was added back to the cocultures and the plate was returned to the incubator for the duration of the experiment. Later, frozen supernatants from multiple experiments were thawed at room temperature and centrifuged to remove any suspended particulate matter. Cytokine specific ELISA kits were then used to test the supernatants for the presence of either IL-10 [Mouse IL-10 Quantikine ELISA Kit; R\&D Systems; Minneapolis, MN, USA] or TGF $\beta$ [Mouse/Rat/Porcine/Canine TGF- $\beta 1$ Quantikine ELISA Kit; R\&D Systems] according to the manufacturer's instructions. 


\section{Statistical Analysis}

Statistical analyses were performed with SigmaPlot 14 [Systat Sofware, Inc; San Jose, CA, USA]. Absorbance values obtained from the $T$ cell suppression assays were analyzed using a one-way ANOVA followed by Holm-Sidak post hoc tests. All comparisons between untreated and aGr1-treated animals were analyzed using a Student's unpaired t-test. Data are presented as means \pm SEM unless otherwise indicated. Correlation strength was determined by calculating the Pearson's coefficient, where R values of 0.7 - 1.0 were considered "Strong" correlations and values between 0.5 - 0.7 were considered "Moderate". Significance of these correlations were determined using these $\mathrm{R}$ values. For all statistical analyses, the significance level was set at $P \leq 0.05$. 


\section{CHAPTER 3: EXPERIMENTAL CHALLENGES \& MODEL DEVELOPMENT}

Several aspects of this project presented some unique experimental challenges that necessitated the adaptation and development of new models. These challenges fell into one of two general categories: culturing ultra low numbers of cells or working with a complex heterogeneous peripheral tissue.

\section{$T$ Cell Proliferation Assays: Adaptations for working with small cell populations.}

Initially we decided to use CFSE dilution to assess T cell proliferation in our mixed cell co-cultures. For these assays splenocytes were processed as described above, then resuspended to $40 \times 10^{6}$ cells $/ \mathrm{mL}$. The CFSE stain was reconstituted at room temperature according to the manufacturer's instructions. A $5 \mu \mathrm{M}$ working solution was made up by adding $5 \mu \mathrm{L}$ of the stock CFSE to $10 \mathrm{~mL}$ sterile HBSS. 1 volume of this solution was added to the splenocytes (i.e. $2 \mathrm{~mL}$ of splenocytes at $40 \times 10^{6}$ cells $/ \mathrm{mL}$ were stained with $2 \mathrm{~mL}$ CFSE working solution). Cells were incubated for 5 minutes in a $37^{\circ} \mathrm{C}$ water bath, removed promptly and incubated another 5 minutes at room temperature, protected from light. The reaction was quenched with enough HBSS-2\% FBS to fill the tube, then mixed thoroughly by pipetting. Cells were centrifuged at $350 \times \mathrm{g}$ for 5 minutes, washed with HBSS-2\% FBS, and spun once more with the same settings. After the final wash, cells were 
resuspended in 5mL RPMI-10\% FBS and counted using the nucleocounter. The splenocytes were then analyzed on the flow cytometer to check if the cells had been sufficiently stained. The percentage of $\mathrm{CD} 4^{+}$and $\mathrm{CD} 8^{+}$splenocytes was also determined at this time. This allowed us to properly resuspend the splenocytes according to number $\mathrm{T}$ cells so that they could be co-cultured with the sorted aMDSCs at a 1:1 ratio of T cells to aMDSCs.

After 5 days, each co-cultured well was stained with CD4 and CD8 so that CFSE dilution could be examined specifically in the T cells. Cells were stained in the 96 well plate, resuspended in $200 \mu \mathrm{L}$ of wash buffer, and transferred to a microtube for analysis. The wells were rinsed once more with $200 \mu \mathrm{L}$ of wash buffer which was also transferred to the corresponding microtube.

Our pilot experiments using this protocol had mixed success due to inconsistent $\mathrm{T}$ cell activation. Without this positive control, we were unable to determine whether or not our adipose-derived cells were affecting $T$ cell proliferation. After verifying our reagents, we concluded that the concentration of T cells in each well was too low to establish a sufficiently robust IL-2 and IL-4driven autocrine feedback loop to sustain a proliferative T cell response. Ultimately it was this observation that motivated our search for an alternate method to detect changes in T cell proliferation that might have increased sensitivity to more subtle changes in T cell numbers. We also worked to optimize our culture system to ensure consistent $\mathrm{T}$ cell activation with each experiment.

Since a demonstrated ability to suppress inflammatory $T$ cell responses is the "gold standard" for MDSC characterization we needed to optimize our culture 
system to ensure consistent $T$ cell activation with each experiment. To this end we tested various combinations of soluble and plate-bound aCD3/aCD28 antibodies at different concentrations. Eventually, we developed a conditional culture system that was based upon the final number of cells in each well (Table 3.1).

Table 3.1: Conditional T Cell Activation Algorithm for Co-cultures.

\begin{tabular}{|c|c|c|}
\hline $\begin{array}{c}\text { Total Cell \# / Well } \\
\text { (aMDSCs + T Cells) }\end{array}$ & $\begin{array}{c}\text { [aCD3] } \\
\text { (Plate-bound) }\end{array}$ & $\begin{array}{c}\text { [aCD28] } \\
\text { (Plate-bound) }\end{array}$ \\
\hline $5000-10000$ & $3 \mu \mathrm{g} / \mathrm{mL}$ & $3 \mu \mathrm{g} / \mathrm{mL}$ \\
\hline$>10000$ & $1 \mu \mathrm{g} / \mathrm{mL}$ & $3 \mu \mathrm{g} / \mathrm{mL}$ \\
\hline
\end{tabular}

Once we had a system in place to consistently induce T cell proliferation, we turned to the problem of measuring the potential suppressive activity of the adipose-derived cells. As the pilot experiments had demonstrated, cytometrybased methods were theoretically possible. In practice however, this turned out to be tedious and extremely time-consuming. More importantly CFSE-dilution was not nearly as sensitive of a detection method when it was scaled down to the level of $6000 \mathrm{~T}$ cells per well. An issue that was further compounded by the fact that these cells were being co-cultured with adipose SVF, which notoriously autofluoresces in the FITC channel (the same channel used to detect CFSE signal). This desire to produce consistently reliable and unambiguous results motivated our search for alternate ways to assess aMDSC-mediated changes in $\mathrm{T}$ cell proliferation.

Eventually we landed on a new form of MTT assay, which preserved cellular viability by producing a soluble dye instead of an insoluble precipitate. This left us 
the option to further analyze the co-cultures using flow cytometry or even to assess T cell activity over time as long as the cells were washed and resuspended in fresh media afterwards. The major advantage of this type of assay however was its sensitivity, as it could provide an accurate assessment of cell number in cultures with as few as 5000 cells per well.

Choosing this method did require a few compromises on our part. For instance, in cytometry-based systems you have the option to stain for cell-specific markers which allows for the identification of the exact cell populations that are proliferating. Since the MTT assay format, at its core, is a semi-quantitative, relative assessment of cell number in each well and is based on their metabolic activity it is impossible to know what cells are contributing to the differences between conditions. This meant that we could not assume that absorbance value differences between the positive controls and the experimental conditions were solely due to changes in T cell proliferation. However, we determined that the loss of this specificity was acceptable as the more traditional $\mathrm{H}_{3}$-thymidine incorporation method suffers from a similar issue in that all proliferating cells are radio-labeled, not just $T$ cells.

The MTT assay format we chose was a water-soluble tetrazolium salt (WST) assay and is based on the activity of cellular dehydrogenases. These essential enzymes are involved in oxidative phosphorylation and use the potential energy of electrons to actively transport hydrogen ions into the intermembrane space of mitochondria. The electrons released during the oxidation reactions are then transferred to an electron mediator such as ubiquinone. This is the step that 
the WST assay takes advantage of. The reagent is added directly to the co-culture supernatant and contains its own water-soluble electron mediator. This molecule scavenges the electrons that typically reduce ubiquinone and transports them extracellularly where they can be used to convert a tetrazolium salt into formazan, a water-soluble dye. As cellular dehydrogenase activity increases, more electrons are released, resulting in an increased production of formazan. Ultimately, this changes the color of the culture supernatant from light yellow to orange, which is then quantified by measuring light absorbance at 450nm. The higher the absorbance value is, the more metabolic activity that is going on in the well, and the more cells in that particular culture condition.

Even with three successful iterations of this MTT-based assay under our belts, we were still concerned about the fact that at its core MTT assays rely on the assumption that all the cells within a given co-culture are metabolizing at a comparable rate. Since we had improved the reliability of our small-scale $T$ cell activation protocol, we decided to try the flow cytometry-based proliferation assays one again in order to verify that the absorbance changes we observed in the MTT assays did result from a true decrease in T cell proliferation. Cell number titrations revealed that we were able to reliably measure CFSE dilution with as few as 5000 MACS-enriched T cells. Additionally, these titrations indicated that Day 3 of the culture would be the optimal time to collect proliferation data as the CFSE dye was completely diluted and undetectable by Day 4 (Figure 3.1). All in all, three T cell suppression assays were performed according to these parameters. None of them however showed that adipose-derived MDSCs (aMDSCs) had any of the 
suppressive activity that was observed in the previous MTT assays (Figure 3.2 a). After comparing the set-up of the MTT and CESE assays we determined that there were two major differences that could have resulted in these discrepancies between the sets of experiments: the kinds of responder cells we used and the length of time the cells were co-cultured.

The original MTT assays used splenocytes while MACS-enriched T cells were used for the CFSE set. Given the diverse array of effector mechanisms at their disposal, it was possible that MDSCs were promoting M2 polarization in splenic macrophages which were then responsible for the observed reduction in $\mathrm{T}$ cell proliferation. To test for this, we compared aMDSC-mediated suppression between MACS-enriched T cells and splenocytes. Once again, we did not see any immunomodulatory activity in the co-cultures with MACS-enriched T cells. Surprisingly, suppressive activity was absent from the splenocyte co-cultures as well (Figure 3.2 b). This implicated the time that these cells spent in co-culture as the source of these discrepancies.

The CFSE-based proliferation assays as well as the assay comparing splenocytes and purified T cells were all analyzed on culture Day 3, while the cocultures in the original set of MTT experiments had been allowed to run through Day 5. To this end, two sets of MDSC co-cultures were set up simultaneously with proliferation being assessed on either Day 3 or 5 . We had to return to the MTT format for those experiments as CFSE would be completely diluted by Day 5 (Figure $3.2 \mathrm{c}$ ). Using these parameters, we observed a striking difference between days 3 and 5 in terms of MDSC-mediated suppression of T cell proliferation. As 
with previous experiments there was little to no suppressive activity on culture Day 3 regardless of whether MDSCs were co-cultured with splenocytes or MACSenriched T cells. By Day 5 however, the PI in MDSC co-cultures was significantly less than one indicating that suppression had occurred (Figure $3.2 \mathrm{c}$ ). As further verification of these results, T cells were counted in each well after absorbance measurements were taken. Here we found that MDSC co-cultures on Day 5 had significantly fewer T cells than the positive controls, while there wasn't a difference between the two culture conditions on Day 3. Besides providing additional optimization parameters for these suppression assays, these troubleshooting experiments gave us valuable insight into aMDSC biology. First, whatever effector mechanism aMDSCs are using does not prevent the initial activation and subsequent proliferation of naïve T cells. Instead it appears to stop proliferation over time, once it has already started. Second, aMDSC-mediated immune modulation can directly affect $T$ cell proliferation without the need for an intermediary cell type such as M2 macrophages (Figure $3.2 \mathrm{~d}$ ).

As the MDSC-mediated suppression could only be detected after 5 days, we were once again faced with the problem of non-specificity. The MTT format is more often used to determine the cytotoxicity of a specific treatment or drug. Traditionally, these cell viability assays use a single cell line making it easy to assume that changes are due to the experimental conditions themselves and not different cell types behaving differently. However, for our suppression assays, there would be two (aMDSCs and MACS-enriched T cells) or more (in the case of splenocytes) different cell types in each co-culture. This heterogeneity meant that 
the results could not be taken at face value, as the metabolic activity of our effector cells could inadvertently be influenced by the presence of activated T cells. To account for this, we developed a secondary method to verify the final number of $T$ cells in each culture condition. On day 5 , following the final MTT absorbance measurement, cells were washed and stained with CD4 and CD8 antibodies. Then the entirety of each individual well was collected at the cytometer in order to calculate the final number of T cells in each co-culture. Finally, we correlated the absorbance values from the MTT assay with the absolute number of $\mathrm{CD} 4^{+} \mathrm{T}$ cell numbers in each well (Figure $4.4 \mathrm{e}$ ). This revealed that there was a strong correlation between these two values $(R=0.9978)$, confirming that this assay could be used to reliably measure both $\mathrm{T}$ cell proliferation and the suppressive potential of adipose-derived MDSCs in a heterogeneous mixture of cells. 
(a)
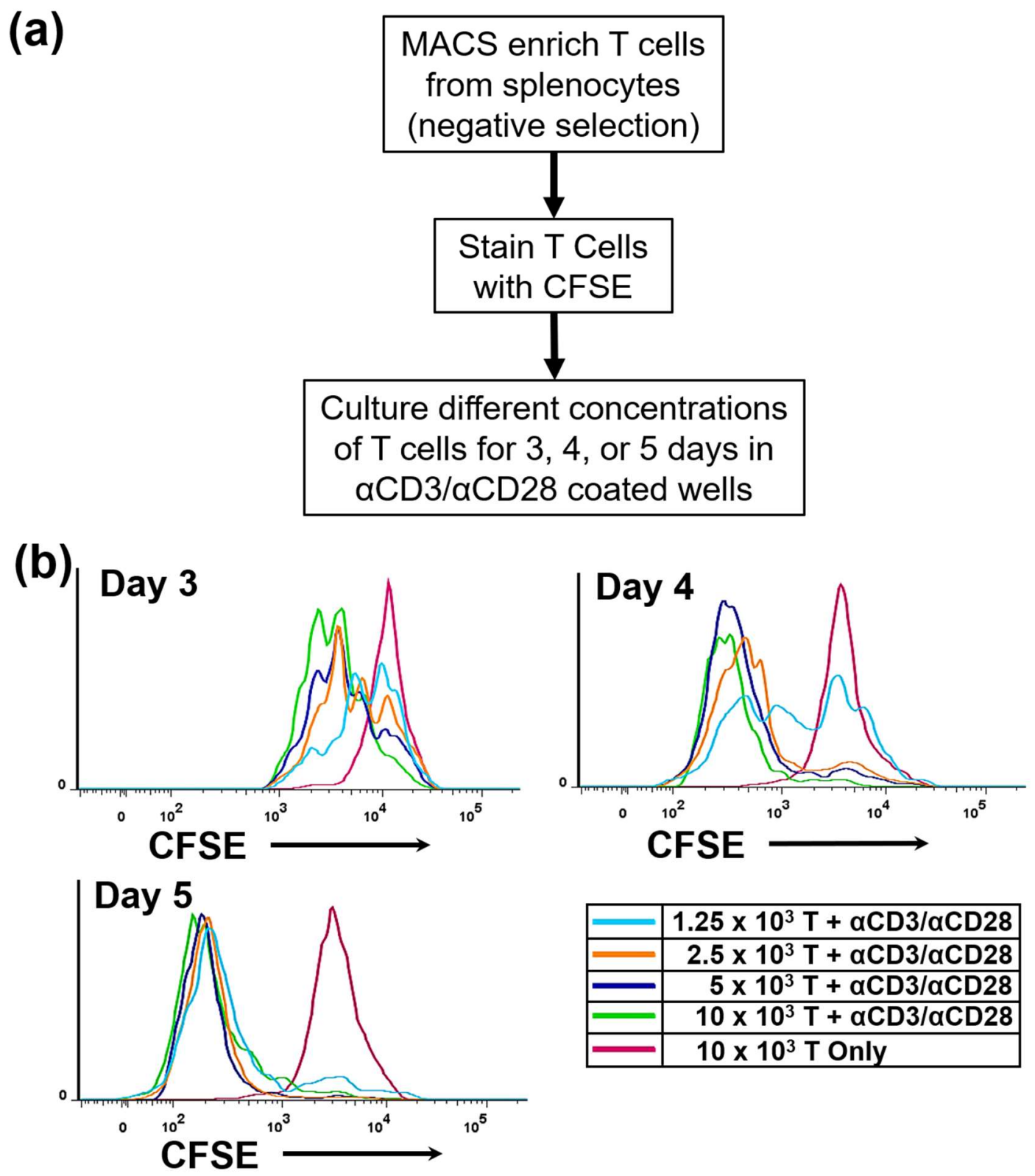

\begin{tabular}{|c|c|}
\hline & $1.25 \times 10^{3} \mathrm{~T}+\alpha C D 3 / \alpha C D 28$ \\
\hline & $2.5 \times 10^{3} \mathrm{~T}+\alpha \mathrm{CD} 3 / \alpha C D 28$ \\
\hline & $5 \times 10^{3} T+\alpha C D 3 / \alpha C D 28$ \\
\hline & $10 \times 10^{3} T+\alpha C D 3 / \alpha C D 28$ \\
\hline & $10 \times 10^{3}$ T Only \\
\hline
\end{tabular}

Figure 3.1: CFSE can be used to measure the proliferation of as few as $\mathbf{2 5 0 0}$ MACS-enriched T cells. (a) Schematic of CFSE titration experimental design. (b) Histograms showing CFSE dilution profiles in aCD3/aCD28-stimulated CD4 $4^{+} \mathrm{T}$ cells after 3,4 , or 5 days. 

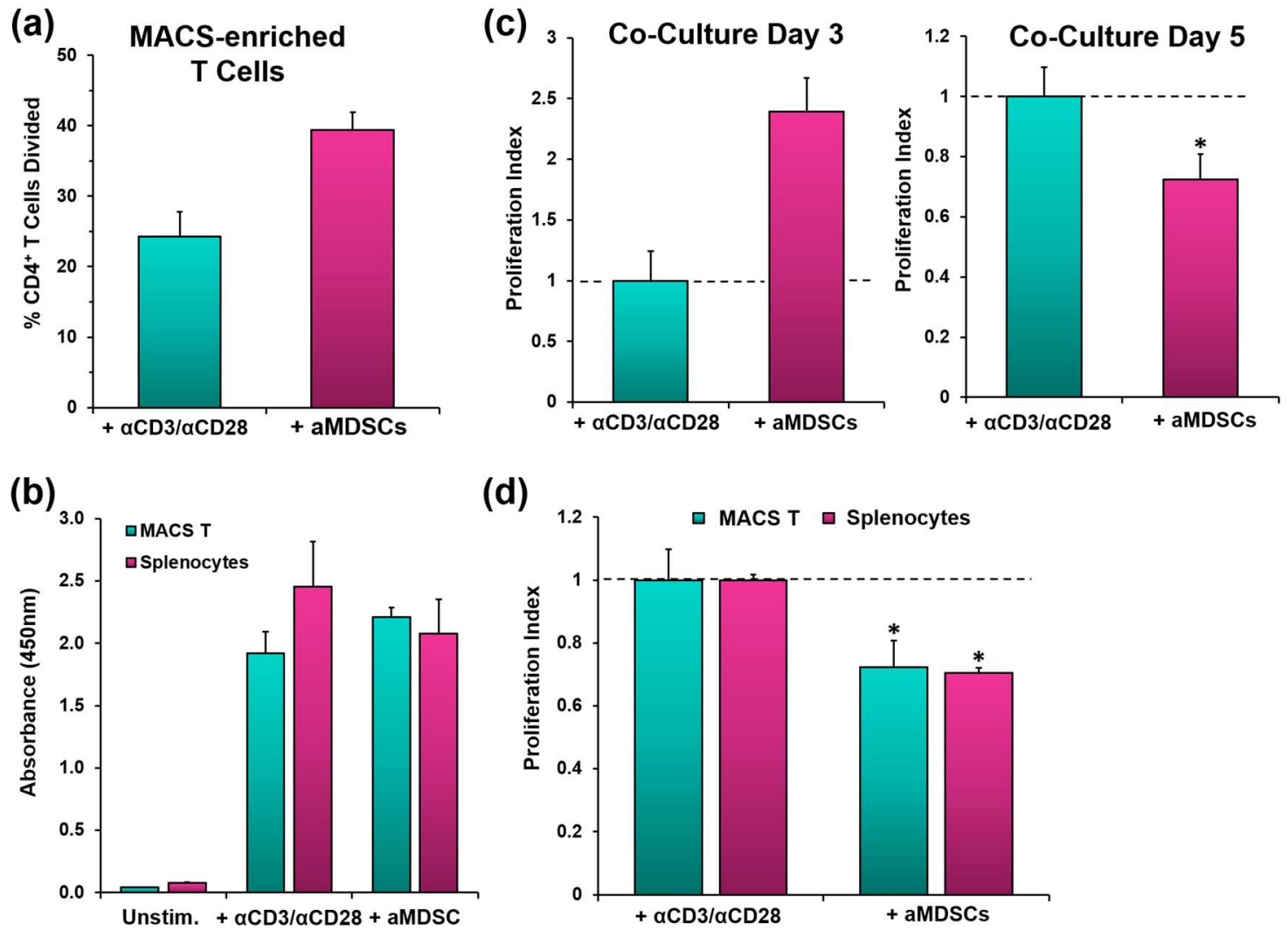

Figure 3.2: MDSCs suppress aCD3/aCD28-stimulated proliferation of splenocytes and MACS-enriched T cells. (a) Naïve, CFSE-stained, MACSenriched T cells were activated with plate-bound $\alpha C D 3 / \alpha C D 28$ and co-cultured with aMDSCs. After 3 days $C D 4^{+} \mathrm{T}$ cell proliferation was assessed by measuring CFSE dilution with flow cytometry. Data is pooled from three independent experiments where each co-culture was performed in triplicate. (b) Naïve splenocytes or MACS-enriched $\mathrm{T}$ cells were activated with plate-bound aCD3/aCD28 and co-cultured with aMDSCs. After 3 days proliferation was assessed via MTT assay. Data derived from one experiment where each coculture condition was performed in triplicate. (c) aCD3/aCD28-stimulated splenocytes were co-cultured aMDSCs for three or five days. Proliferation was assessed at each time point via MTT assay. Data derived from one experiment where each co-culture condition was performed in triplicate. (d) Naïve splenocytes or MACS-enriched T cells were activated with plate-bound aCD3/aCD28 and cocultured with aMDSCs. Proliferation was assessed after five days via MTT assay. Data pooled from two independent experiments where each condition was performed in triplicate. 


\section{Accommodations for the Unique Nature of Adipose Stromal Cells}

\section{Flow Cytometry Gating Strategies}

As this was the first time that anyone had specifically looked at the impact of $\alpha \mathrm{Gr} 1$ depletion on healthy adipose, we were uncertain as to what kinds of changes we could expect in terms of immune cell dynamics. We hypothesized that MDSC depletion in vivo would increase signs of overt inflammation in the tissue. However, we were unsure as to the exact ways in which this might manifest. We developed multi-color staining panels for flow cytometry to identify and characterize a broad range of cell types, classically associated with obesity-related AT inflammation.

Some of the earliest charges that occur at the cellular level in obesity take place in the macrophage and $T$ cell compartments [141, 142], so we chose to analyze each sample with two distinct staining panels that targeted some of the key phenotypic features of each of these cell types. The MDSC/Macrophage panel included some of the classic markers associated with the "M2" to "M1" phenotypic switch that occurs in the early stages of obesity [21, 29] (Figure 3.3 a). Occasionally, due to a shortage of the CD301 or Ly6G antibodies, an alternative gating strategy was used to define adipose tissue macrophages (Figure $3.3 \mathrm{~b}$ ) and MDSCs (Figure 3.4 a). There was no difference between these two strategies in the final number of MDSCs found within the tissue (Figure $3.4 \mathrm{~b}$ ). As for the panel designed to detect changes in T cell dynamics, we included markers indicative of T cell activation in case MDSC depletion elicited an inflammatory response similar to what is observed in obesity. This panel also included markers that could be used 
to distinguish between various $\mathrm{T}$ cell subsets (i.e. regulatory, effector memory, and central memory $\mathrm{T}$ cells) which are sensitive to changes in the inflammatory state of AT [39] (Figure 3.5). 

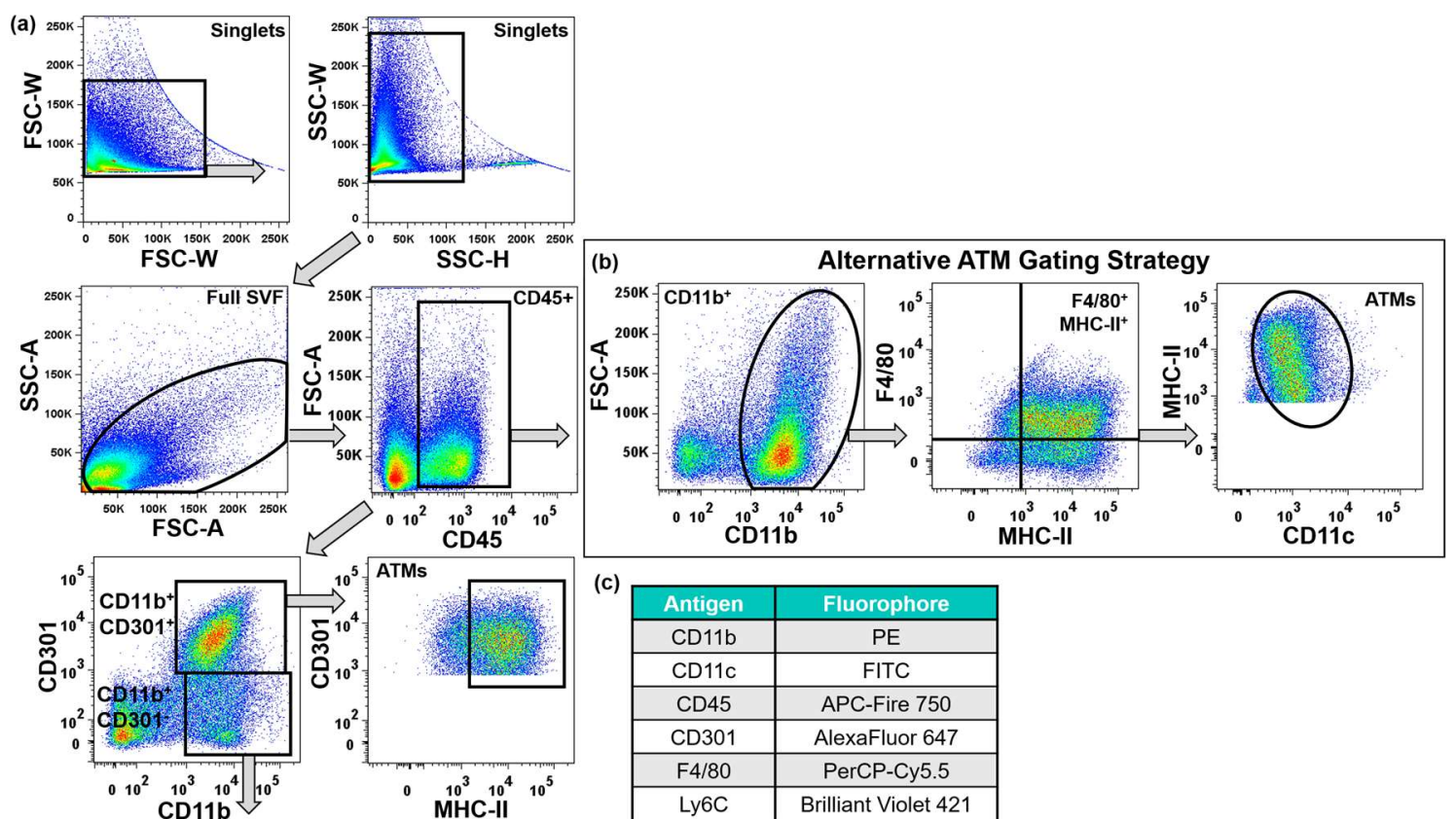

(c)

\begin{tabular}{|c|c|}
\hline Antigen & Fluorophore \\
\hline CD11b & PE \\
\hline CD11c & FITC \\
\hline CD45 & APC-Fire 750 \\
\hline CD301 & AlexaFluor 647 \\
\hline $\mathrm{F} 4 / 80$ & PerCP-Cy5.5 \\
\hline Ly6C & Brilliant Violet 421 \\
\hline Ly6G & Brilliant Violet 605 \\
\hline MHC-II & PE-Cy7 \\
\hline
\end{tabular}
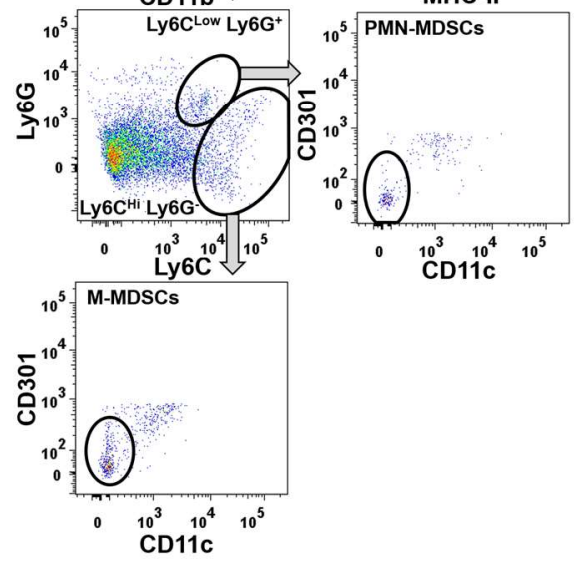

Figure 3.3: SVF Myeloid Cell Gating Strategy (a) Gating strategy used to characterize MDSCs and adipose tissue-resident macrophages (ATMs) in AT SVF (b) Alternate ATM gating strategy used when CD301 was not present in staining panel (c) Cell markers and the fluorophores used to characterize myeloid cell populations in the spleens and AT SVF of C57BI/6 mice. 
(a)
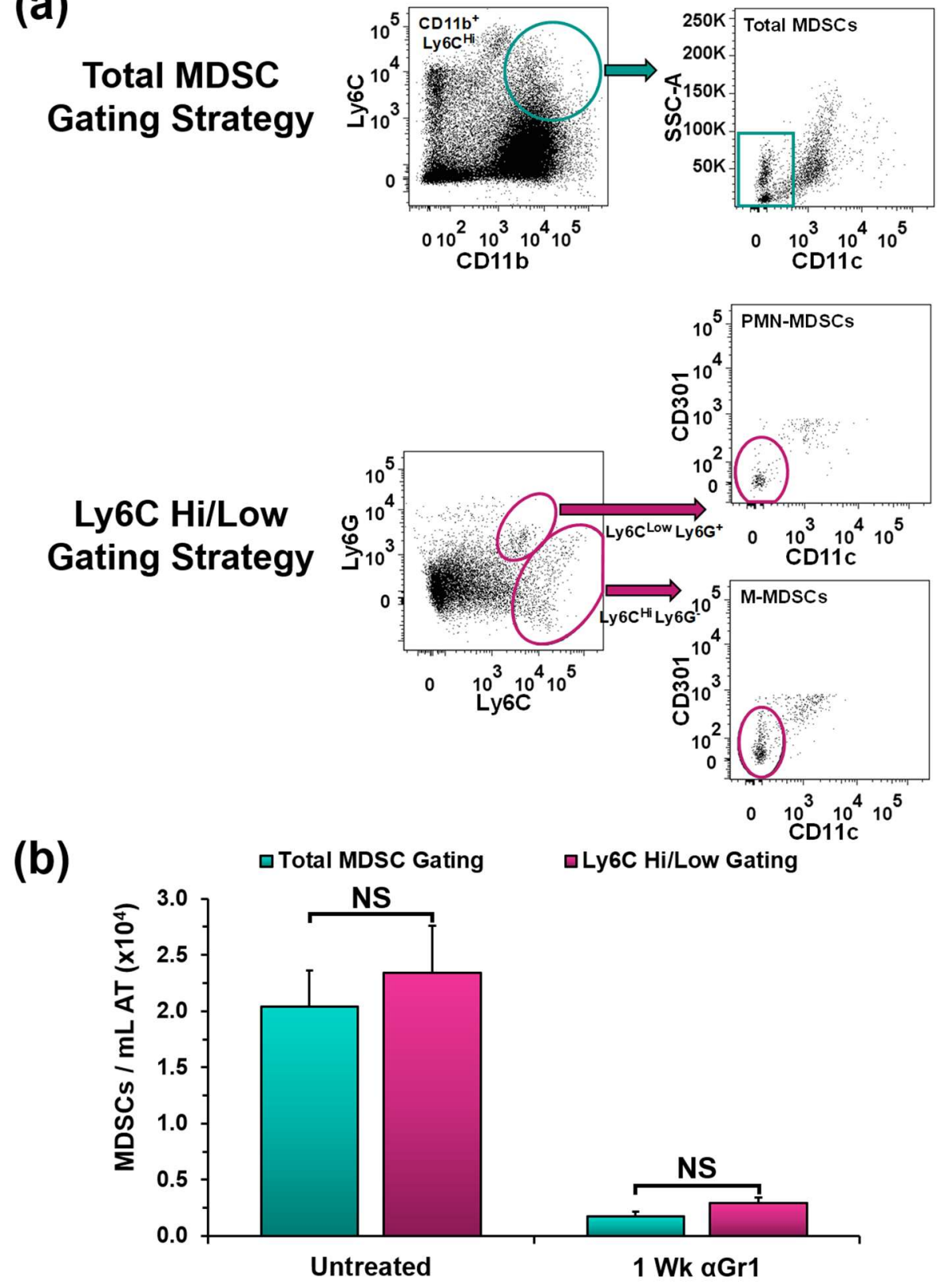

Figure 3.4: Alternative MDSC Gating Strategy (a) Representative dot plots depicting the gating strategies used to quantify adipose-resident MDSCs based on the absence (Upper) or presence (Lower) of Ly6G and CD301 in the staining panel. The Ly6C vs Ly6G dot plot used in the "Ly6C Hi/Low" gating strategy was previously gated on the total $\mathrm{CD} 45^{+} \mathrm{CD} 11 \mathrm{~b}^{+} \mathrm{CD} 301^{-}$SVF cells. The CD11b vs Ly6C dot plot shown in the "Total MDSC" gating strategy was previously gated on CD45 ${ }^{+}$SVF cells. (b) Absolute number of MDSCs found in the SVF of untreated or aGr1-treated mice using either the "Total MDSC" (Blue) or "Ly6C Hi/Low" (Pink) gating strategies. The numbers shown for the "Hi/Low Gates" gating strategy were obtained by combining both M-MDSC and PMN-MDSC populations. Bars depict the mean of 11 untreated or 81 -week aGr1-treated mice. Error bars depict SEM. 
(a)
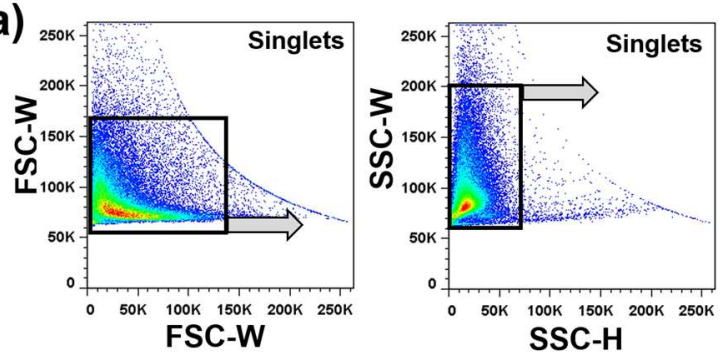

(b)

\begin{tabular}{|c|c|}
\hline Antigen & Fluorophore \\
\hline CD4 & PE-Cy5 \\
\hline CD8 & Brilliant Violet 510 \\
\hline CD44 & APC-Cy7 \\
\hline CD62L & FITC \\
\hline CD69 & Brilliant Violet 605 \\
\hline Foxp3 & PE \\
\hline IL33R (STS2) & APC \\
\hline
\end{tabular}
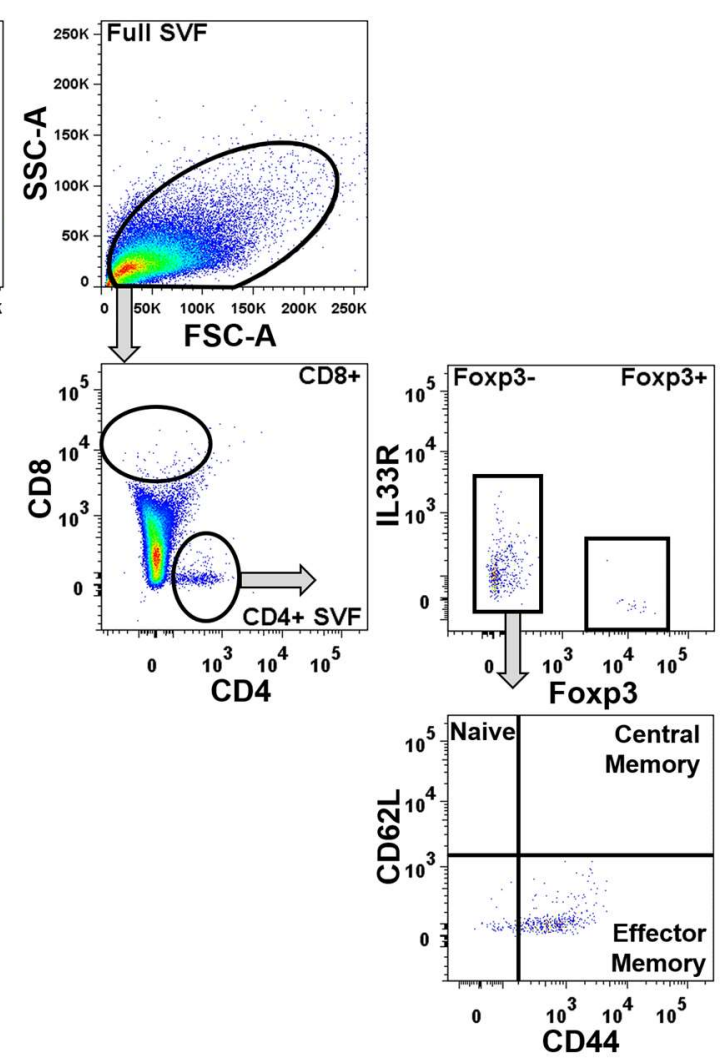

Figure 3.5: T Cell Phenotype \& Activation State Gating Strategy. (a) Gating strategy used to examine T cell populations in AT SVF. (b) Cell markers and the fluorophores used to characterize myeloid cell populations in the spleens and AT SVF of C57BI/6 mice. 


\section{Adjusting for Adipose Autofluorescence}

Once the panels were designed, the cells harvested and the data collected, we faced an additional challenge on top of our not knowing what specific cell types, if any, would change as a result of the $\alpha \mathrm{Gr} 1$ treatment. Adipose tissue is inherently autofluorescent [143]. This is a problem that plagues any researcher wanting to use fluorescence-based technology such as flow cytometry or confocal microscopy to tease apart the inner workings of this tissue. Multiple factors give adipose tissue and the SVF cells isolated from it their natural fluorescent qualities.

One major factor is the dense extracellular matrix (ECM) that acts as the scaffolding that holds adipocytes in place and allows the stromal cells to travel throughout the tissue. A large portion of the ECM is composed of collagen fibers. The uniform, repetitive structure of this multi-unit protein predisposes it to vibrating at resonance frequencies $[144,145]$. As a result, certain wavelengths of light cause these subunits to absorb enough energy that an election jumps up one energy level. As this energy dissipates, the election falls back to its original energy state consequently emitting a different wavelength of light [144].

The extracellular matrix in adipose tissue is a major contributor to its natural fluorescence. This dense network of collagen and elastin fibers is an essential scaffold that holds adipocytes in place and allows stromal cells to travel throughout the tissue. Collagen fibrils are the primary structural proteins of any ECM and adipose is no exception [146, 147]. Each fibril is composed of three long $\alpha$-helical subunits which twist together to form the final triple helical quaternary structure or this protein. The amino acid sequences are strikingly regular, exhibiting a fairly consistent pattern of Glycine-Proline-X, where X can be any amino acid [146]. 
The identity of this third residue can influence the fluorescent nature of the final protein [144]. This feature is mostly observed with the inclusion of any of the aromatic amino acids: tryptophan, tyrosine, or phenylalanine. Structurally each of these molecules contains at least one benzene ring. Molecular ring structures, such as these, are uniquely able to retain their stability upon absorbing light energy. The absorbed photon excites electrons in the molecule causing them to jump to a higher energy state, but as this energy dissipates the elections fall back to their original energy level [148]. This leads to the emission of photons at a lower wavelength. Most collagen types contain a non-negligible number of tyrosine residues [146]. Collagen type VI, found in adipose [149], contains both tyrosine and phenylalanine residues, further enhancing the innate autofluorescence of the ECM in this tissue.

In addition to aromatic amino acids within individual collagen fibrils, protein crosslinking can enhance a tissue's autofluorescent qualities. For instance, a fluorescent compound known as pyridinoline forms as a byproduct of structural crosslinking between collagen and elastin fibrils during the formation and maturation of extracellular matrices in tendons and bones [145].

The final factor contributing to the auto fluorescence of our adipose SVF cells may actually result from the collagenase digestion itself. A recent study analyzed how collagenase I digestion altered the structural and fluorescent nature of collagen I [150]. They found that the innate fluorescence of this protein increased in a dose dependent manner following exposure to, collagenase I. Additionally, they determined that this was likely caused by an increase in the 
exposure of tyrosine residues that have otherwise been shielded from direct light exposure by the triple helical structure of the intact protein.

Since we know that the digestion process only breaks down the collagen fibers but does not fully dissociate their connections to the cells themselves, it is very possible that the autofluorescence we observe in our SVF isolates stems, to some degree, from exposed tyrosine and phenylalanine residues on partially digested cell-associated collagen.

Along with ECM-associated autofluorescence, adipose-resident macrophages also contributed to the spectral interference we encountered in our flow cytometric analysis of SVF samples. ATM autofluorescence is thought to be directly related to their functional activities within the tissue. For instance, one study found that macrophages with upregulated iNOS expression and activity were more fluorescent in the 488 channel than those expressing lower amounts of this enzyme [143]. They postulated that this increased fluorescence was likely caused by an increased concentration of the cofactors required for iNOS activity as 3 out of these 5 compounds have autofluorescent properties that emit light is the 488 $\mathrm{nm}$ range. This same study also found that ATM autofluorescence increased proportionally with increased lipid uptake by these cells. They ascribed this lipidinduced autofluorescence in ATMs to the high fat diet condition since they phagocytose the lipids released when overburdened adipocytes pyropoptose. A more recent study however found that ATMs in healthy mice also actively participate in lipid uptake and release, potentially acting as intermediaries between the adipocytes and systemic circulation [25]. 
The natural fluorescent qualities of adipose tissue proved to be one of the most troublesome aspects of our flow cytometry experiments, primarily affecting our ability to accurate analyze our data by complicating the compensation process. We sought to reduce these issues as much as possible at every stage of the experiment. For instance, as we developed our staining panels, we tried to avoid using FITC or Alexa Fluor 488 conjugated antibodies since this is the major channel in which AT autofluoresces (Figure 3.6 a). Some of our panels had as many as 8 colors however, so this was not always possible. In these cases, the software generated compensation matrices were often thrown off by the inherent FITC positive aspects of our unstained controls. Additionally, some of the markers in our more exploratory panels were exclusionary or expressed at such low levels that using SVF cells for our single-color controls was not feasible. To ensure that we collected the best data possible and that we were able to accurately analyze these results we chose to use compensation beads.

As an additional control we used the fluorescence minus one (FMO) technique to correct for the data spread that occurs when multiple colors are combined into the same staining panel. This allowed us to identify the true line between negative and positive cell populations in these multi-parameter experiments.

Although each of these strategies helped to minimize the impact of autofluorescence on the data collection portion of each experiment, they were still only partially effective and the autofluorescent nature of adipose tissue and SVF cells was still measurable in most of our samples. Our primary means of correcting 
this spectral overlap was by designing our own compensation matrices in the FlowJo analysis software (Figure 3.6 b). For each set of experiments, I would try to keep this matrix as consistent as possible between replicates. This was relatively easy to do as long as the data for each fluorophore was collected at the same PMT voltages as all the other replicates. Instead of building a new matrix from scratch or even having designing one based on my single-color compensation beads in FlowJo itself, I would often use the acquisition defined matrix as my starting point and adjust the overlap values as needed.

I was able to determine what values required adjustment by looking at the dot plots of a fully stained sample and an unstained sample. I would then look at the plot for each fluorophore combination. Primarily I wanted to see that the displayed populations were distributed in a "natural", round shape as opposed to forming a diagonal line which is indicative of under compensation. I also adjusted overly compensated parameters which appeared as diagonal populations that extended beyond 0 for that fluorophore.

The main concern when making compensation matrices by hand is overly adjusting the spectral overlap to the point that you inadvertently create false positive or negative cell populations. In my compensation process I paid particular attention to these potential artifacts and avoided definitively gating on populations that drastically shifted intensity with small charges in overlap values. Altogether by employing these strategies at each stage of the experiment we were successfully able to produce consistent and reliable data from multi-parametric flow cytometry 
experiments despite the inherent autofluorescence of adipose resident macrophages and SVF cells in general. 
(a)

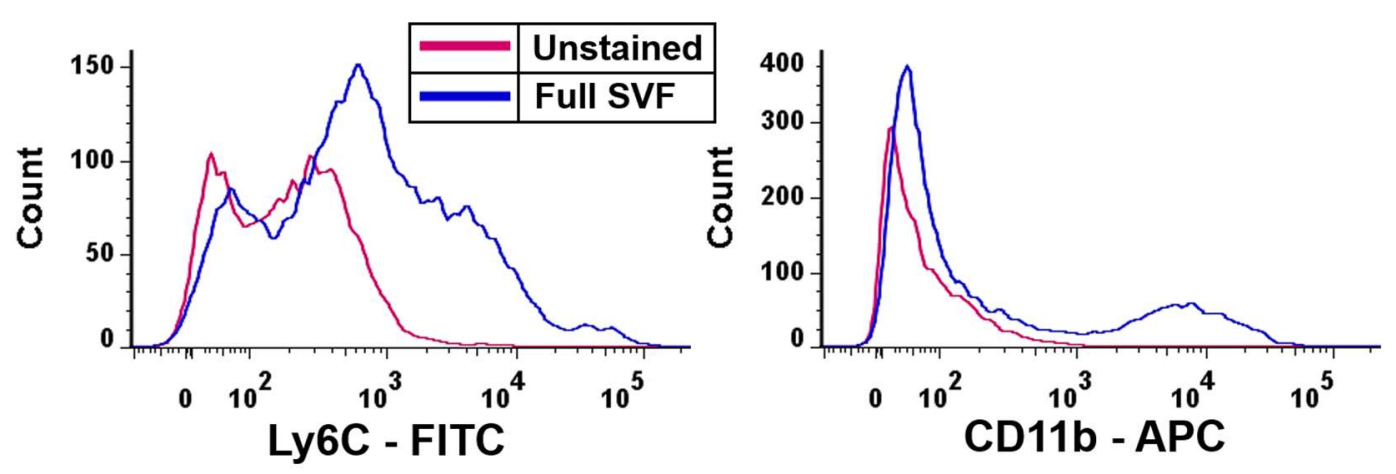

(b)

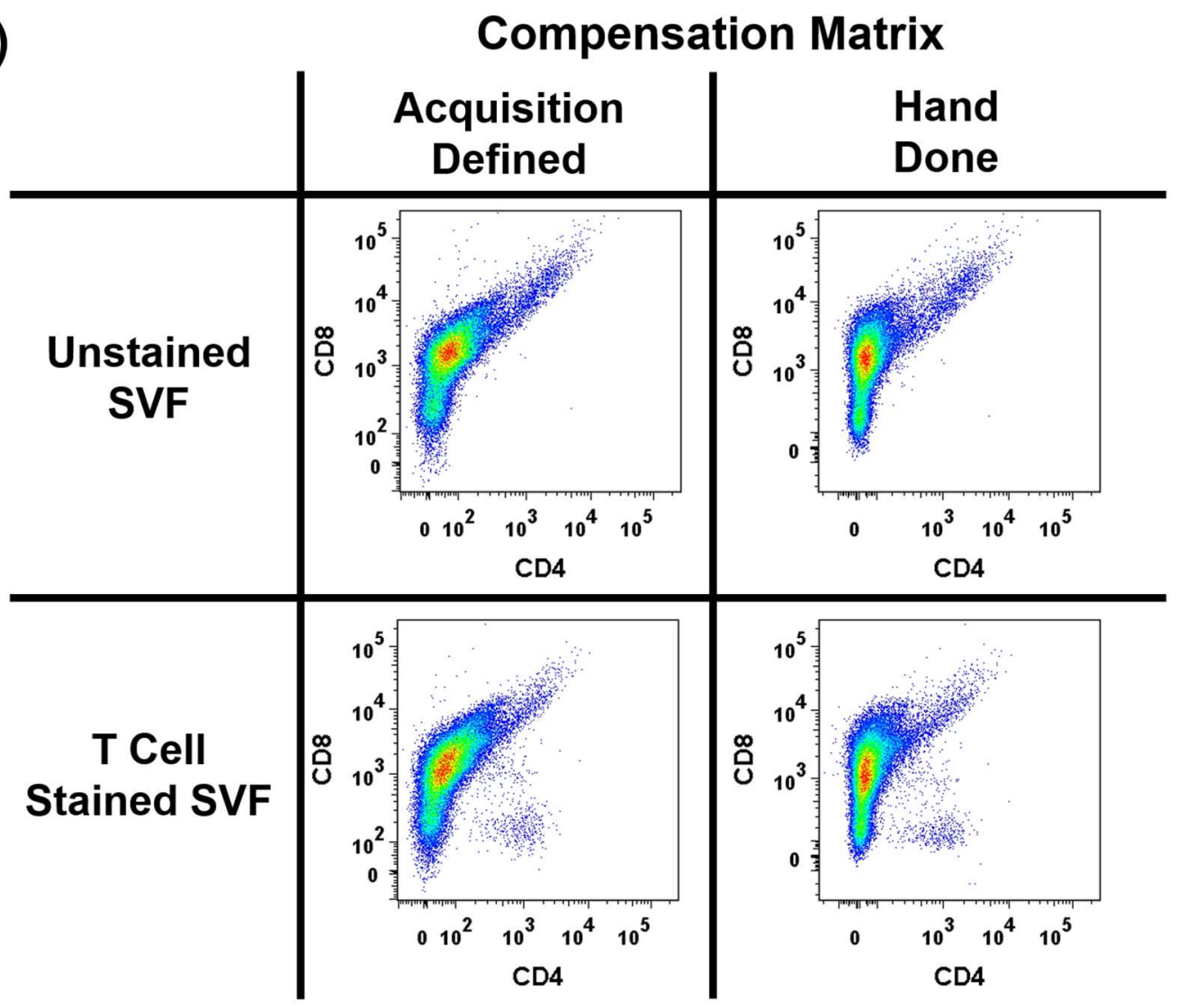

Figure 3.6: Flow cytometry analysis of SVF requires manually adjusting spectral overlap values to account for the natural autofluorescence of AT. (a) Representative histograms comparing FITC or APC fluorescence in full SVF samples that were either left unstained (Pink) or stained for myeloid cell markers (Blue) (b) Representative dot plots depicting SVF samples that were either left unstained (Upper Row) or stained for T cell markers (Lower Row). SVF cells isolated from the same animal were used for both the unstained and stained samples. Data shown in the left column uses the compensation matrix calculated from the DIVA software and single-color stained compensation beads. The righthand column shows the same dot plots after correcting for autofluorescence. 


\section{CHAPTER 4: BONA FIDE MYELOID-DERIVED SUPPRESSOR CELLS IN HEALTHY ADIPOSE TISSUE}

Previous work in our lab has examined the therapeutic value of the adipose tissue (AT) stromal/vascular cellular fraction (SVF) in improving vascular function $[85,151,152]$. In one study the SVF isolate lost many of its beneficial effects when the $\mathrm{CD}_{11 \mathrm{~b}^{+}}$cells were depleted [85]. Further characterization of these therapeutically relevant CD $11 b^{+}$cells found that approximately $45 \%$ of were also $\mathrm{Gr} 1^{+}$. Similar populations of $\mathrm{CD} 11 \mathrm{~b}^{+} \mathrm{Gr} 1^{+}$cells have been observed by others in the AT of healthy, control animals $[129,153]$, yet no detailed characterization of these cells has been performed to date. Given the immunomodulatory nature of the adipose tissue stromal cells in healthy mice we hypothesized that these $\mathrm{CD} 11 \mathrm{~b}^{+} \mathrm{Gr} 1^{+}$cells were an adipose-resident population of immunosuppressive, myeloid-derived suppressor cells (MDSCs).

\section{There are $C D 11 b^{+}$Ly6 $C^{H i / L o w}$ Ly6G ${ }^{-/+} C D 301^{-}$cells in healthy adipose tissue.}

In order to determine if these $\mathrm{CD}_{11} \mathrm{~b}^{+} \mathrm{Gr} 1^{+}$cells could be immunomodulatory, myeloid-derived suppressor cells (MDSCs), we first developed a detailed panel of surface markers to characterize the myeloid cell populations resident in healthy adipose tissue (AT). Ly6C and Ly6G were included as well as AT-resident macrophage markers F4/80, CD301, and MHC-II to differentiate neutrophils and polymorphonuclear-MDSCs (PMN-MDSCs) from adipose tissue macrophages, dendritic cells, and monocytic-MDSCs (M-MDSCs). 
CD11 b ${ }^{+}$CD301- CD11c- Ly6C HilLow Ly6G $^{+/-}$MDSC-like cells made up $0.77 \pm 0.07 \%$ of the SVF harvested from healthy AT. $85.73 \pm 1.8 \%$ of these were Ly6C ${ }^{H i}$ Ly6G- $^{-}$ M-MDSC-like cells $(0.63 \pm 0.09 \%$ of the total SVF), while Ly6CLow Ly6G+ PMNMDSC-like cells accounted for the remaining $14.28 \pm 1.76 \%$ of the CD11 $\mathrm{b}^{+} \mathrm{CD} 301^{-}$ CD11 c cells $(0.12 \pm 0.03 \%$ of the total SVF) (Figure $4.1 \mathrm{a}-\mathbf{b})$. Neither population of the cells we identified expressed traditional adipose macrophage or dendritic cell markers such as CD301, F4/80, or CD11c; however, the Ly6C ${ }^{H i}$ Ly6G- cells $^{-1}$ did exhibit heterogeneous expression of MHC-II (Figure $4.1 \mathrm{c}$ ).

PMN-MDSCs can be difficult to distinguish phenotypically from CD11 $\mathrm{b}^{+}$ $\mathrm{Ly}_{6 \mathrm{G}}{ }^{+}$neutrophils. Cells with this surface marker combination only account for $0.15 \pm 0.03 \%$ of the total SVF $\left(3.72 \times 10^{3} \pm 0.77\right.$ cells $\left./ \mathrm{mL} \mathrm{AT}\right)$ with the majority falling into the $\mathrm{CD} 11 \mathrm{~b}^{+}$Ly6C $\mathrm{C}^{\mathrm{Low}} \mathrm{Ly}_{6 \mathrm{G}}{ }^{+}$population $\left(67.3 \pm 7.0 \%\right.$ of total $\mathrm{Ly} 6 \mathrm{G}^{+}$cells) (Figure 4.2). Furthermore, we believe that these $\mathrm{Ly} 6 \mathrm{G}^{+}$cells are likely to share more functional characteristics with PMN-MDSCs than with neutrophils since other groups have shown that there are very few neutrophils in healthy adipose tissue $[154,155]$.

Overall, the surface markers expressed by the $\mathrm{Ly}_{6 \mathrm{C}^{\mathrm{Hi}}} \mathrm{Ly}_{6 \mathrm{G}}{ }^{-}$and Ly6C $\mathrm{C}^{\mathrm{Low}}$ Ly6G ${ }^{+}$SVF populations are comparable to those found on tumor-associated MMDSCs and PMN-MDSCs, respectively [102]. Since the Ly6C ${ }^{+}$Ly6G- cells $^{-}$ represented a majority of the candidate $C D 11 b^{+}$CD301- CD11 $\mathrm{c}^{-}$population, we decided to focus our subsequent characterization studies on this subset. 
(a)

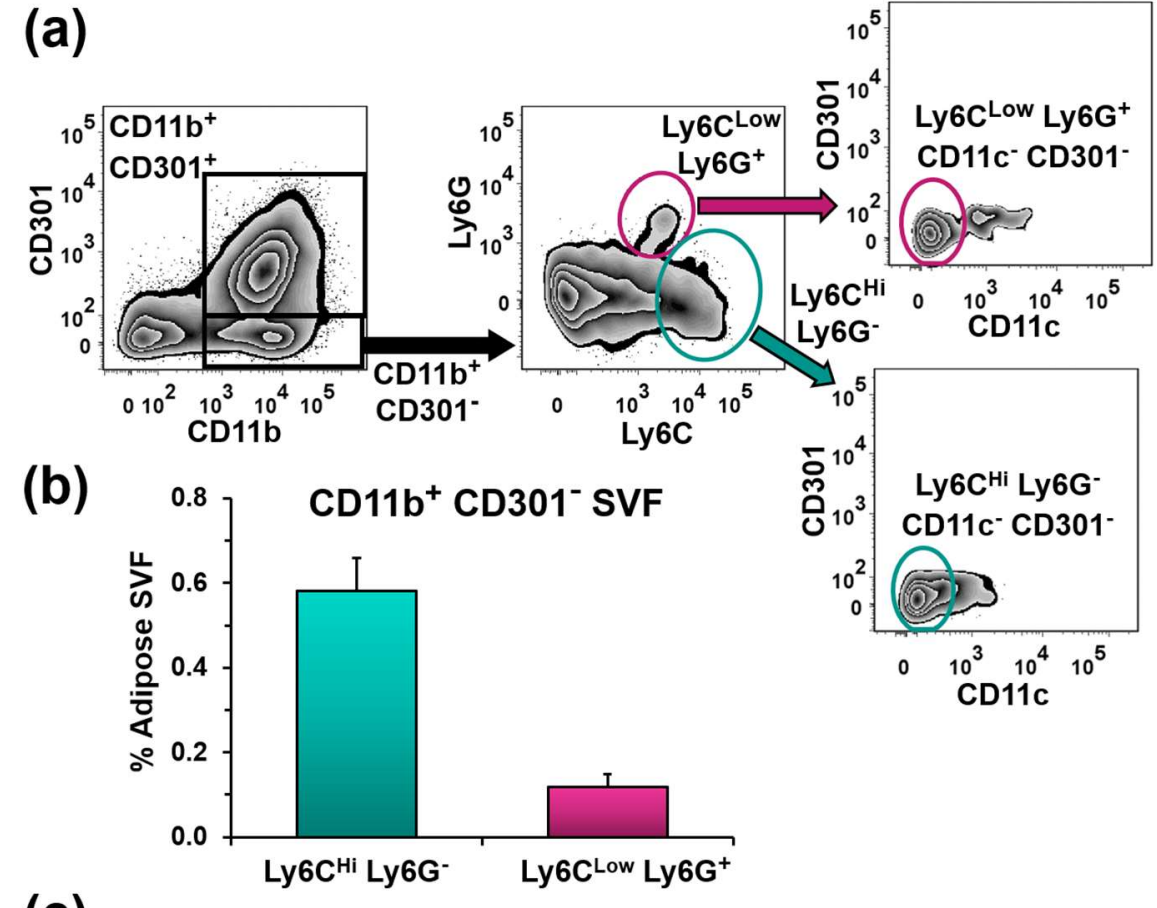

(c)

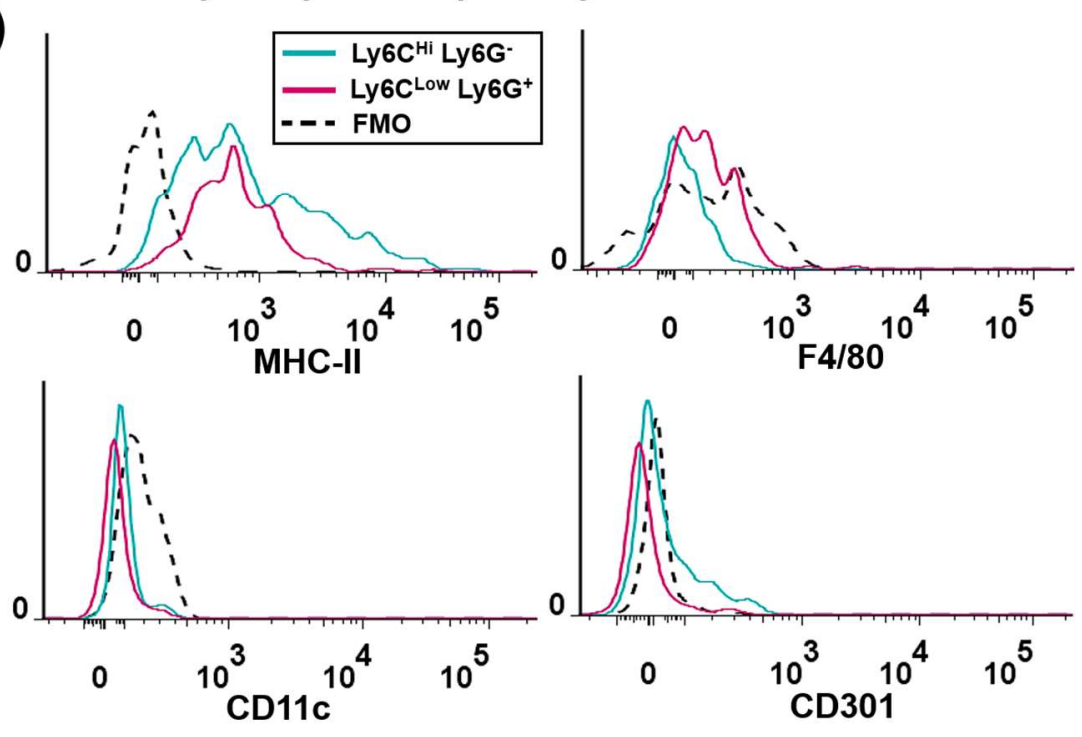

Figure 4.1: CD11 ${ }^{+}$CD301- Ly6C $^{\text {Hillow }} \mathrm{Ly}^{6} \mathrm{G}^{+/}$- cells are in adipose tissue from metabolically healthy mice. (a) Representative zebra plots depicting the gating strategy used to characterize the myeloid cell populations in adipose tissue from individual, healthy mice. (b) Quantification of the CD11 b ${ }^{+}$CD301- populations in the stromal vascular cell fraction (SVF) isolated from healthy AT. Adipose from 11 mice analyzed individually. Error bars represent SEM. (c) Representative histograms depicting surface expression of macrophage markers by CD11 $\mathrm{b}^{+}$ CD301- Ly6CHi Ly6G- cells (Blue line), Ly6CLow Ly6G+ cells (Pink line), or Fluorescence-minus one (FMO) controls (dashed line) in healthy AT. Selected histograms depict median expression within the group of 11 individuals analyzed. 

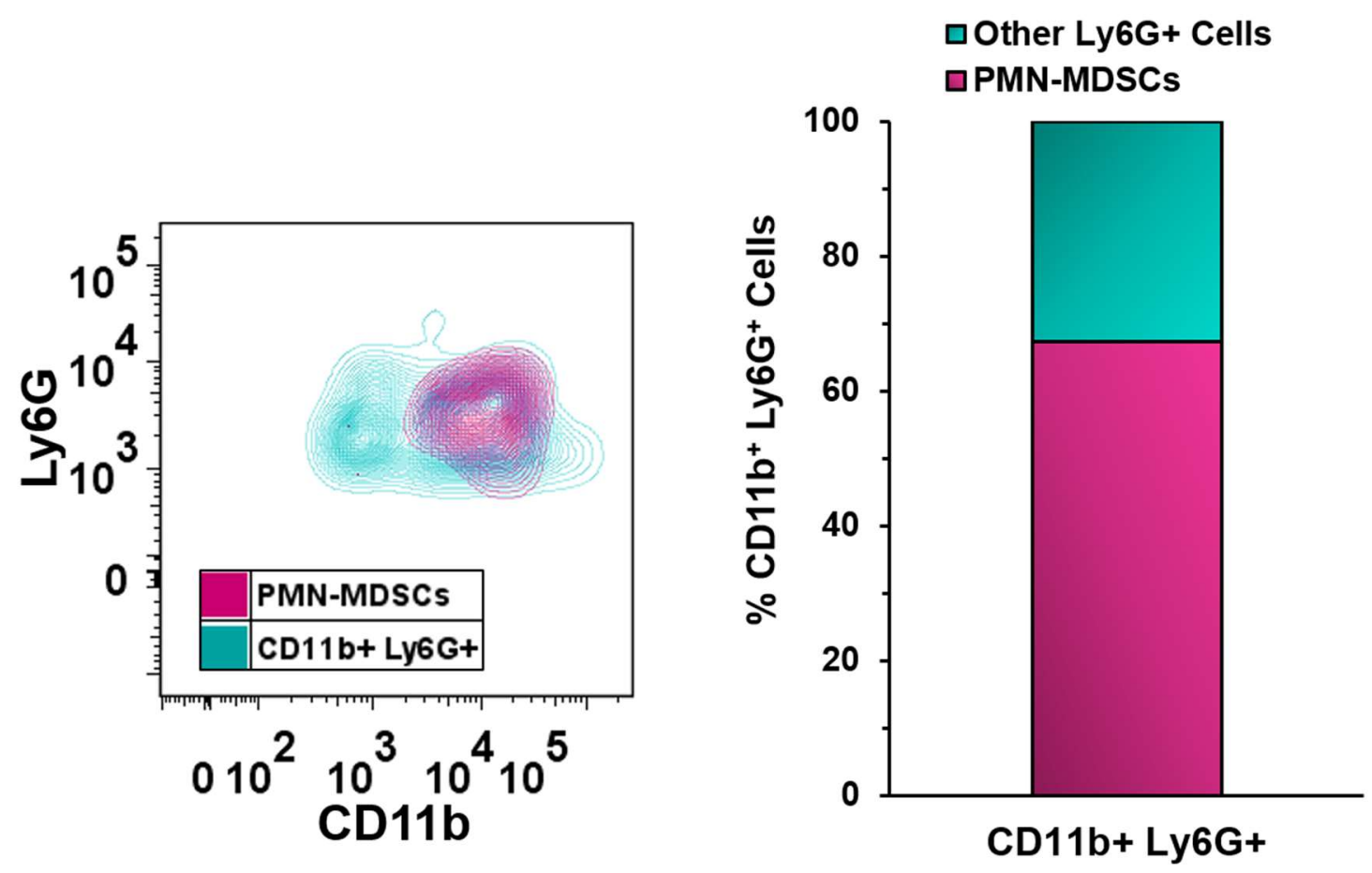

Figure 4.2: PMN-MDSCs make up the majority of $\mathrm{Ly6G}^{+}$cells in healthy adipose tissue. (Left) Overlaid contour plots showing the portion of CD11 $\mathrm{b}^{+}$ $\mathrm{Ly}_{6 \mathrm{G}}{ }^{+}$cells that are PMN-MDSCs in the SVF of healthy mice. (Right) The frequency of different cell subsets within the CD11b+ $\mathrm{Ly}^{+} \mathrm{G}^{+}$SVF population. Adipose from 4 mice analyzed individually. 


\section{Adipose-resident $C D 11 b^{+} L^{2} 6 C^{H i}$ Ly6G- cells express canonical MDSC- associated genes.}

Simply expressing surface markers reminiscent of tumor-derived MDSCs is not sufficient evidence to definitively characterize these adipose-resident cells as a genuine population of MDSCs. Because we only recovered $9-10 \times 10^{3} \mathrm{M}$ MDSC-like cells per $\mathrm{mL}$ of AT with each sort we were forced to limit the number of genes we could probe for. In the tumor microenvironment MDSCs can be distinguished from normal monocytes by their co-expression of immunoregulatory molecules and cytokines such as arginase (Arg1), inducible nitric oxide synthase (Nos2), VEGF, PGE 2, IL-4R, IL-10, and TGF $\beta$ [100]. We focused our analysis on Arg1, Nos2, II10, and Tgfb1 as these genes are considered "crucial" for MDSC biology by Bronte et al (2016) [100]. Traditionally, Nos2 and Arg1 expression are used to distinguish "M1" from "M2" macrophages. In tumors however coexpression of these two enzymes is considered a classic feature of MDSCs. Further differentiating MDSCs from monocytes is their active transcription of antiinflammatory cytokines such as IL-10 and TGF $\beta$.

To determine if our adipose-derived $\mathrm{Ly}_{6} \mathrm{C}^{\mathrm{Hi}}$ Ly6G- cells were actively transcribing these MDSC-associated genes, we isolated RNA from sorted CD11 $\mathrm{b}^{+}$ Ly6C $^{\text {Hi }}$ Ly6G- SSC Low cells and performed qRT-PCR. Transcriptional analysis of

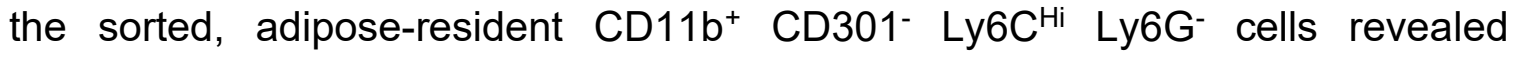
abundant transcripts of $\operatorname{Arg} 1$, Nos2, and Tgfb1 (Figure 4.3 a). They also expressed low levels of I/10 (Figure $4.3 \mathrm{a}$ ).

Intriguingly we also found that these adipose-resident cells were actively transcribing II33 (Figure 4.3 a). This cytokine plays an important role in regulating 
the immunomodulatory phenotypes of several of the key cell types integral to the homeostatic immune network in healthy AT. The cellular source of IL-33 in adipose tissue has yet to be identified [31], so finding active transcription of this cytokine in our sorted cells definitely raises some interesting questions worth future investigations.

Combined with their surface marker phenotype, the gene expression profile of these CD11 $\mathrm{b}^{+} \mathrm{CD} 301^{-} \mathrm{Ly}_{6 \mathrm{C}^{\mathrm{Hi}}} \mathrm{Ly} 6 \mathrm{G}^{-}$cells further highlights their similarity to the Ly6C ${ }^{H i}$ Ly6G- M-MDSCs found in tumors and at sites of chronic inflammation [100]. Furthermore, when compared with adipose tissue macrophages, the $\mathrm{Ly}^{6} \mathrm{C}^{\mathrm{Hi}} \mathrm{Ly}^{\mathrm{H}} \mathrm{G}^{-}$ cells exhibited a distinct expression pattern for immunomodulatory genes such as Arg1, Tgfb1, and I/10 (Figure 4.3 b), suggesting that these cells may have a unique role within the AT immune cell network. 
(a)

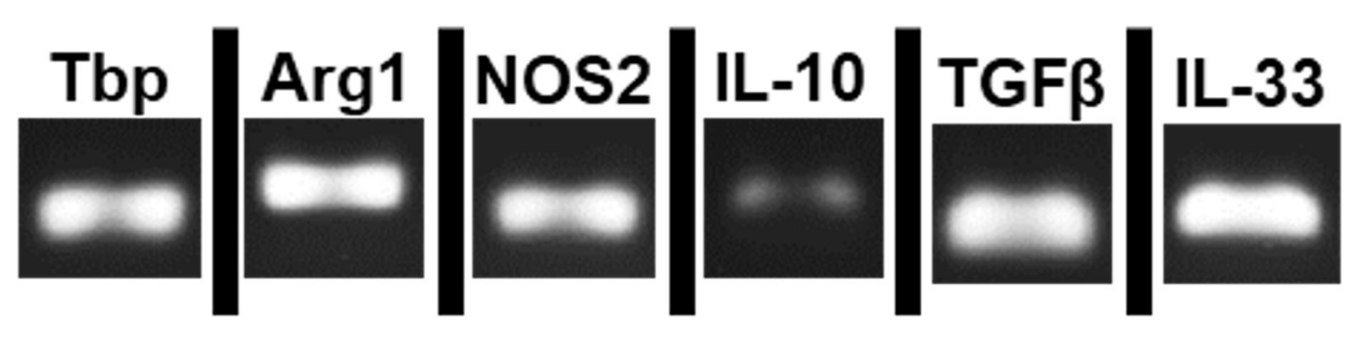

(b)

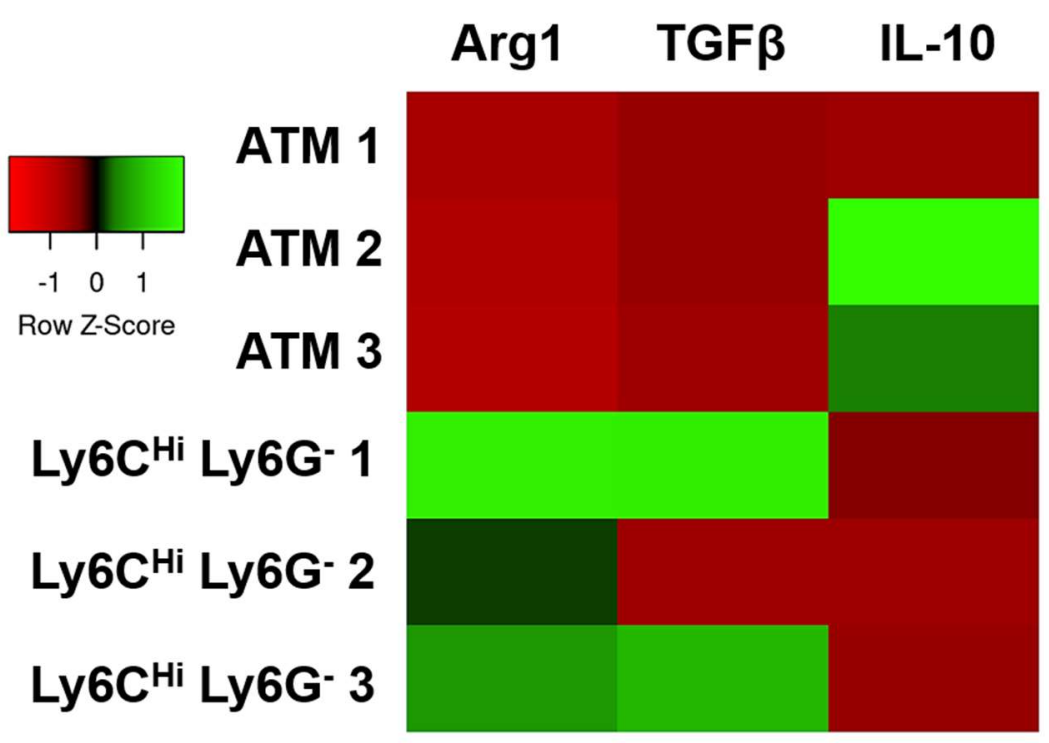

Figure 4.3: Gene expression in Sorted CD11 ${ }^{+}$CD301- Ly6C ${ }^{H i}$ Ly6G- cells. (a) Representative bands from an agarose gel showing active transcription of MDSCassociated genes in FACS-isolated $\mathrm{CD}^{11 b^{+}} \mathrm{Ly}_{6 \mathrm{C}^{\mathrm{Hi}}} \mathrm{Ly} 6 \mathrm{G}^{-}$cells. Data is representative of three independent experiments with AT of 10 mice pooled for each experiment. Depicted bands are from the same gel where PCR amplification of each gene was tested in duplicate. Gene amplification confirmed using Tbp as a housekeeping gene and primer specificity confirmed with non-template controls (NTC). (b) Heat map depicting relative expression of Arg1, Tgfb1, and I/10 by FACS-isolated adipose tissue macrophages (ATMs) or CD11 b+ $\mathrm{Ly}^{+} \mathrm{C}^{\mathrm{Hi}} \mathrm{Ly} \mathrm{G}^{-}$ cells. Each row represents one independent experiment where AT from 5-10 mice was pooled and mRNA was collected from the indicated cell types. Tbp was used as the housekeeping gene for each experiment. 


\section{$C D 11 b^{+} L y 6 C^{+}$Ly6G- $A T$ cells suppress activated $T$ cell responses.}

Given that healthy adipose tissue is constitutively inflamed [13] and contains a network of tissue-resident immunomodulatory cells similar to what is found in the tumor microenvironment, we hypothesized that the CD11 $\mathrm{b}^{+} \mathrm{CD} 301^{-} \mathrm{Ly}^{-\mathrm{C}^{\mathrm{Hi}}} \mathrm{Ly}^{-} \mathrm{G}^{-}$ adipose cells were bona fide, immune suppressive MDSCs. The "gold standard" for differentiating MDSCs from monocytes or neutrophils is their ability to suppress activated T cell proliferation [100]. To test this, CD11 b+ CD301- Ly6C ${ }^{+}{ }^{-1}$ Ly6G $^{-}$cells were sorted from the adipose stromal cells of metabolically healthy mice and cocultured at a 1:1 ratio with $a C D 3 / \alpha C D 28$ activated splenocytes for 5 days. The use of traditional flow cytometry or radiation-based proliferation assays was not feasible, as the small number of $\mathrm{CD} 11 \mathrm{~b}^{+} \mathrm{CD} 301^{-} \mathrm{Ly} \mathrm{C}^{\mathrm{Hi}} \mathrm{Ly} 6 \mathrm{G}^{-}$cells obtained from each sort ( 9-10 $\times 10^{3}$ M-MDSC-like cells / mL of AT) made it difficult to reliably obtain repeatable results with these standard methods. As an alternative, $\mathrm{T}$ cell proliferation in these co-cultures was assessed using a colorimetric MTT assay (Figure 4.4 a). When co-cultured with the isolated Ly6C ${ }^{H i}$ Ly6G- $^{-}$cells and stimulated with plate-bound aCD3/aCD28 antibodies, we found that activated splenocyte proliferation was significantly attenuated relative to stimulated splenocytes alone (PI: $0.38 \pm 0.15$ ) (Figure 4.4 b).

We also co-cultured these adipose-derived cells with MACS-enriched T cells in order to verify that they were directly affecting $T$ cell proliferation, and not promoting the immune suppressive functions of another cell type present in the heterogeneous splenocyte isolate which might, in turn, suppress the T cells. Once again, we found that co-stimulated T cells did not proliferate to the same extent as 
those cultured on their own when they were co-cultured with sorted CD11 $\mathrm{b}^{+}$ CD301- Ly6C ${ }^{\text {Hi }}$ Ly6G- cells (PI: $0.22 \pm 0.04$ ) (Figure 4.4 c-d).

Additionally, we correlated the absorbance values from the MTT assay with the absolute number of $\mathrm{CD} 4^{+} \mathrm{T}$ cells in each well, as determined by flow cytometry, following 5-day co-culture. We found that the positive control cultures had the most $\mathrm{CD}^{+} \mathrm{T}$ cells as well as the highest absorbance values while wells where aCD3/aCD28-stimulated T cells were co-cultured with Ly6C ${ }^{H i}$ Ly6G- $^{-}$cells had both significantly lower absorbance values and fewer $\mathrm{CD} 4^{+} \mathrm{T}$ cells, confirming that this assay can reliably measure $T$ cell proliferation $\left(R^{2}=0.9957\right)$ (Figure 4.4 e). Altogether this suggests that these adipose-resident Ly6C ${ }^{H i}$ Ly6G- $^{-}$cells suppress activated T cell proliferation.

These CD11 $b^{+}$CD301- Ly6C ${ }^{\text {HilLow }}$ Ly6G $^{-}$cells exhibit many of the requisite characteristics of myeloid-derived suppressor cells that accumulate around tumors or in chronic inflammatory conditions such as the ability to suppress activated T cell proliferation and the co-expression of arginase and iNOS. As such, we believe that they are a novel population of MDSCs resident in healthy adipose tissue. 

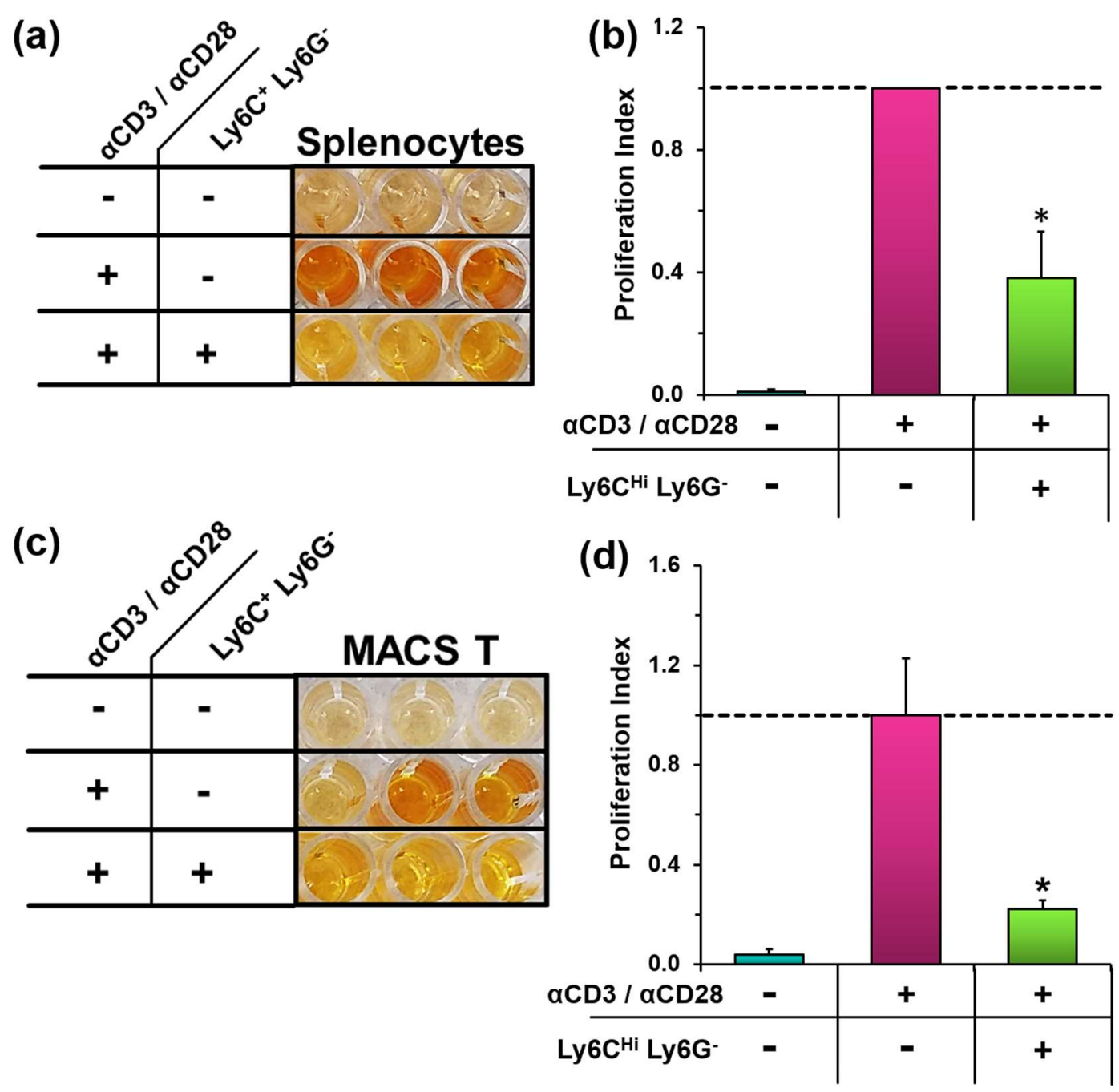

(e) $\quad$ Unstim. $\diamond+\alpha C D 3 / \alpha C D 28 \diamond+$ Ly6C ${ }^{H i}$ Ly6G-Cells

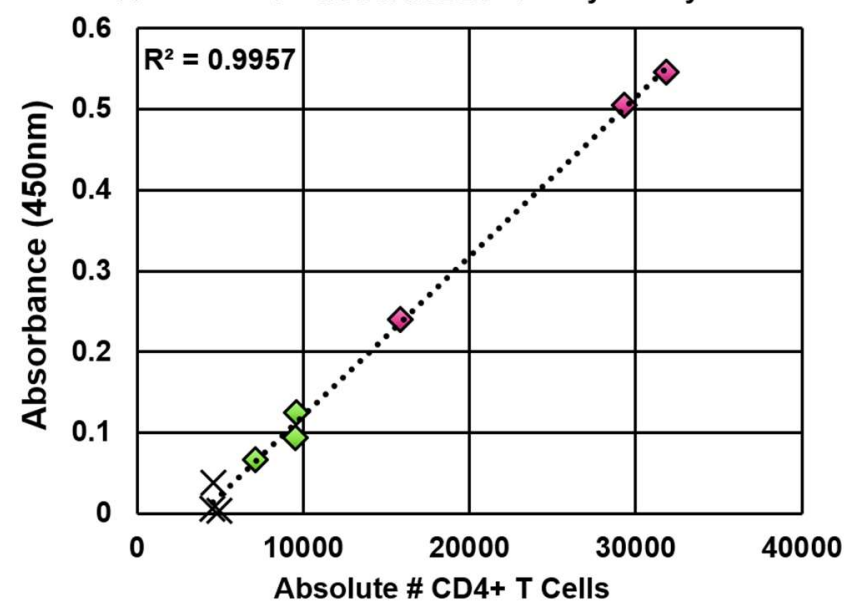


Figure 4.4: Sorted CD11 ${ }^{+}$CD301- Ly6C ${ }^{H i}$ Ly6G- cells suppress aCD3/aCD28 activated $\mathbf{T}$ cell proliferation. $(\mathbf{a}-\mathbf{b})$ Naïve splenocytes were cultured by themselves, activated with plate-bound $\alpha \mathrm{CD} 3 / \mathrm{\alpha CD} 28$, or activated and co-cultured

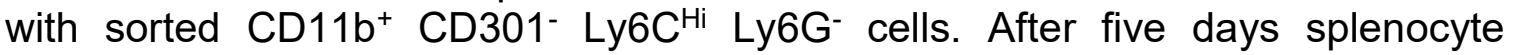
proliferation was assessed via MTT assay. (a) Representative MTT results. Wells depicting each culture condition were selected from a single experiment. (b) aCD3/aCD28-stimulated splenocyte proliferation in each co-culture condition was assessed after five days by measuring absorbance (Abs) at $450 \mathrm{~nm}$. Abs values within each experiment were normalized to create a proliferation index $(\mathrm{PI})$ relative to the mean Abs of aCD3/aCD28-stimulated splenocytes. Bars represent the mean $\mathrm{PI}$ from five independent experiments where each culture condition was performed in triplicate. Error bars represent SEM. Splenocyte co-cultures MTT plate $\mathbf{( c - d )}$ Naïve, MACS-enriched T cells were either unstimulated, activated with platebound $\alpha \mathrm{CD} 3 / \mathrm{aCD} 28$, or activated and co-cultured with sorted CD11 $\mathrm{b}^{+} \mathrm{CD} 301^{-}$ Ly6 $\mathrm{C}^{\mathrm{Hi}}$ Ly6G-cells. After five days T cell proliferation was assessed via MTT assay. (c) Representative MTT assay results. Wells depicting each culture condition were selected from a single experiment. MACS: Magnetic-Activated Cell Separation. (d) aCD3/aCD28-stimulated $T$ cell proliferation was assessed after five days by measuring Abs at $450 \mathrm{~nm}$. Abs values within each experiment were normalized to create a PI relative to the mean Abs of $\alpha C D 3 / \alpha C D 28$-stimulated MACS-enriched $\mathrm{T}$ cells. Bars represent the mean $\mathrm{PI}$ from one of two independent experiments where each culture condition was performed in triplicate and the error bars depict the SEM among those triplicate wells. Differences between positive control cultures and co-cultures with $\mathrm{CD} 11 \mathrm{~b}^{+} \mathrm{CD} 301^{-} \mathrm{Ly} 6 \mathrm{C}^{\mathrm{Hi}} \mathrm{Ly} 6 \mathrm{G}^{-}$cells determined using a Student's $t$-test. (e) Correlation plot comparing the absolute number of $\mathrm{CD} 4^{+} \mathrm{T}$ cells as assessed by flow cytometry and the absorbance at $450 \mathrm{~nm}$ in each well after being co-cultured for five days. Each marker represents one well from one experiment where each condition was performed in triplicate. $R^{2}$ value derived from linear regression. Unstim.: Unstimulated T cells. 


\section{Potential aMDSC Suppression Mechanisms}

Given the similarities between the tumor and healthy adipose tissue microenvironments we wanted to determine if adipose-resident MDSCs (aMDSCs) employed immune modulation techniques similar to those of tumor-derived MDSCs, such as sequestering essential nutrients, inducing $T$ cell apoptosis, or producing anti-inflammatory cytokines. As we were developing our proliferation/suppression assay protocols, we performed some pilot experiments using CFSE-stained splenocytes. Much to our chagrin, we often found no measurable differences in CFSE dilution between the positive controls and our aMDSC co-cultures (Figure 4.5 a), suggesting that these cells were not immunosuppressive. However, when we counted the total number of T cells in each well, we found that there was a greater number of $\mathrm{CD} 4^{+} \mathrm{T}$ cells in co-cultured conditions $\left(8.74 \times 10^{3} \pm 0.82\right.$ cells/well $)$ after 5 days, than in the unstimulated controls $\left(4.69 \times 10^{3} \pm 0.13\right.$ cells/well $)$ suggesting that aMDSCs did not prevent the initial activation and proliferation of naïve splenocytes. However, the aMDSCcontaining co-cultures did have fewer $\mathrm{CD}^{+} \mathrm{T}$ cells on day 5 than the

aCD3/aCD28-activated splenocyte, positive control cultures $\left(25.66 \times 10^{3} \pm 4.97\right.$ cells/well) (Figure 4.5 b).

These seemingly contradictory suppression results were eventually reconciled through the use of the MTT assay format. Even though CFSE dilution proved to be an unsuitable means of measuring aMDSC-mediated immune suppression, these early experiments did provide some helpful data concerning the immunomodulatory mechanism of these cells. T cells co-cultured with sorted aMDSCs were clearly proliferating, as indicated by the observed CFSE dilution 
however, it appeared as if they did not proliferate to the same extent as those activated and cultured alone, suggesting that aMDSCs were preventing their continued proliferation but not their initial activation. This pattern was consistent with two forms of MDSC immune modulation: 1) MDSCs directly induced apoptosis in activated T cells or 2) promoted T cell senescence or exhaustion. In the following section I briefly summarize some of the experiments we performed to explore this question and discuss some of the potential insights that they offer into how aMDSCs modulate proliferative $\mathrm{T}$ cell responses. 


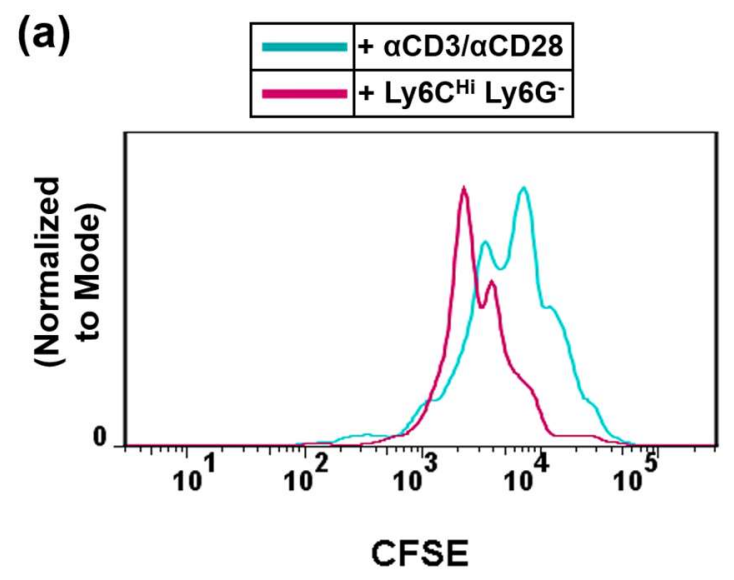

(c)

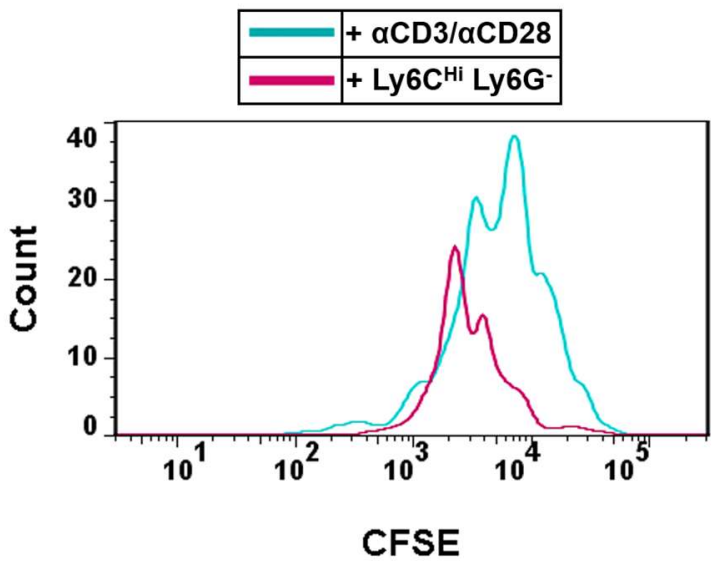

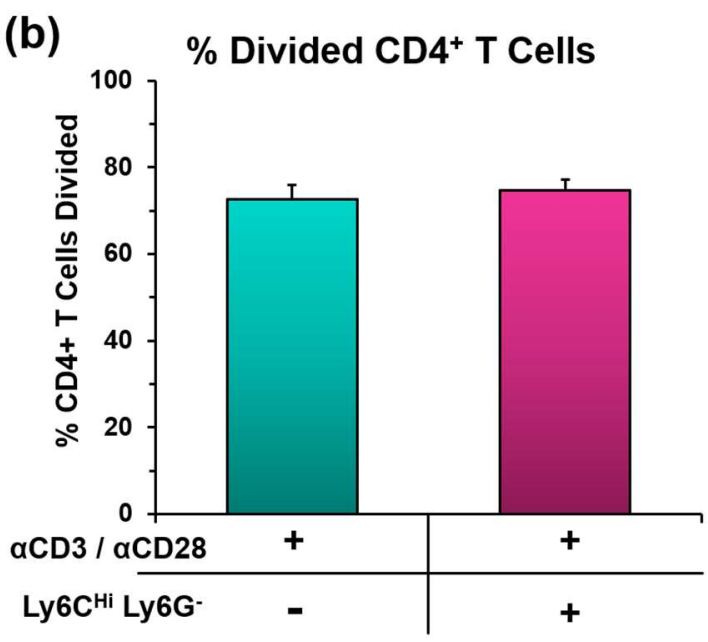

(d)

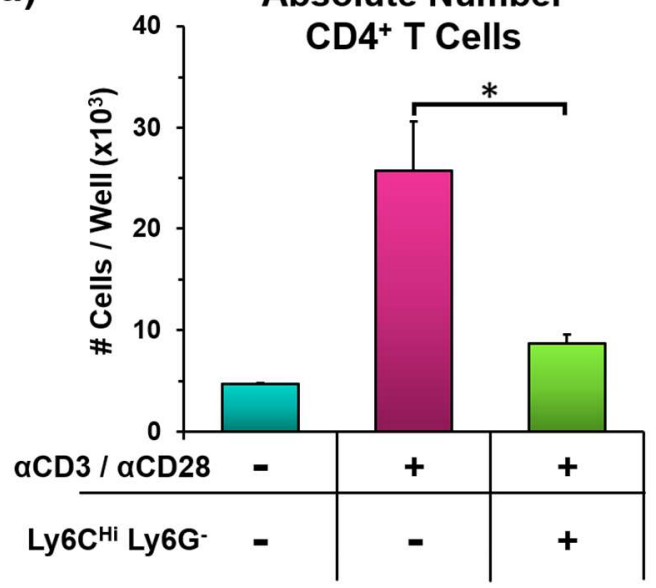

Figure 4.5: Compared to controls, co-culture with aMDSCs reduces the overall number of $T$ cells, without impeding their initial activation. (a) CFSE dilution profile in aCD3/aCD28-stimulated $\mathrm{CD}^{+}$splenocytes following 5-day culture alone (Blue) or with CD11 $\mathrm{b}^{+} \mathrm{CD} 301^{-} \mathrm{Ly} \mathrm{C}^{\mathrm{Hi}}$ Ly6G- cells (Pink). Histograms normalized to mode. (b) \% CD4 ${ }^{+} \mathrm{T}$ cells in each co-culture condition as calculated by FlowJo Proliferation algorithm. (c) CFSE dilution in aCD3/aCD28-stimulated $\mathrm{CD}^{+}$splenocytes following 5-day culture alone (Blue) or with $\mathrm{CD} 11 \mathrm{~b}^{+} \mathrm{CD} 301^{-}$ Ly6 $\mathrm{C}^{\mathrm{Hi}}$ Ly6G- cells (Pink). Histograms displaying raw cell numbers in each division peak. (d) The absolute number of $\mathrm{CD} 4^{+} \mathrm{T}$ cells in each well after splenocytes were cultured alone or stimulated with plate-bound aCD3/aCD28 and cultured with or without sorted CD11 $\mathrm{b}^{+} \mathrm{CD} 301^{-} \mathrm{Ly} 6 \mathrm{C}^{\mathrm{Hi}}$ Ly6G- cells for five days. Bars represent the mean number of cells in each well from one of four independent experiments where each culture condition was performed in triplicate and the error bars depict the SEM among those triplicate wells. 


\section{Apoptosis Induction: aMDSCs do not induce apoptosis in aCD3/aCD28 activated splenocytes and $\mathrm{T}$ cells.}

First, we investigated aMDSC-mediated cell death using our co-culture system, as an increased rate of $\mathrm{T}$ cell apoptosis in aMDSC co-cultures could explain the significantly reduced number of $\mathrm{CD} 4^{+} \mathrm{T}$ cells we observed on day 5 . As before, we set up co-cultures of $\alpha C D 3 / \alpha C D 28$ activated splenocytes with sorted aMDSCs. After 5 days we assessed proliferation via MTT assay. We then stained the cells in each well with CD4, CD8, Annexin V, and 7-AAD to quantify the number of live, apoptotic, or necrotic T cells in each culture condition via flow cytometry. We found that $67.3 \pm 6.9 \%$ of $\mathrm{CD} 4^{+} \mathrm{T}$ cells were in the early or late stages of apoptosis, $23.6 \pm 3.3 \%$ were still viable, and $9.1 \pm 3.6 \%$ were necrotic in the positive controls (Figure 4.6). Intriguingly, co-culture with adipose-derived MDSCs did not alter this distribution, with $68.3 \pm 8.3 \%$ of the $\mathrm{CD} 4^{+} \mathrm{T}$ cells in some stage of apoptosis, $23.0 \pm 4.8 \%$ viable, and only $8.7 \pm 3.5 \%$ necrotic $\mathrm{CD}^{+} \mathrm{T}$ cells respectively (Figure 4.6). This suggests that the aMDSCs were not promoting apoptosis in the activated T cells.

Since the rate of apoptosis was the same between aMDSC co-cultures and our positive controls co-culture with aMDSCs we hypothesized that these cells were using a more indirect method of suppression and began to pursue other MDSC-associated immunomodulatory techniques that could account for the delayed onset suppression we were seeing. To this end, we focused our subsequent investigations on the nutrient deprivation, cytokine production, and oxidative stress mechanisms discussed in the Introduction. 

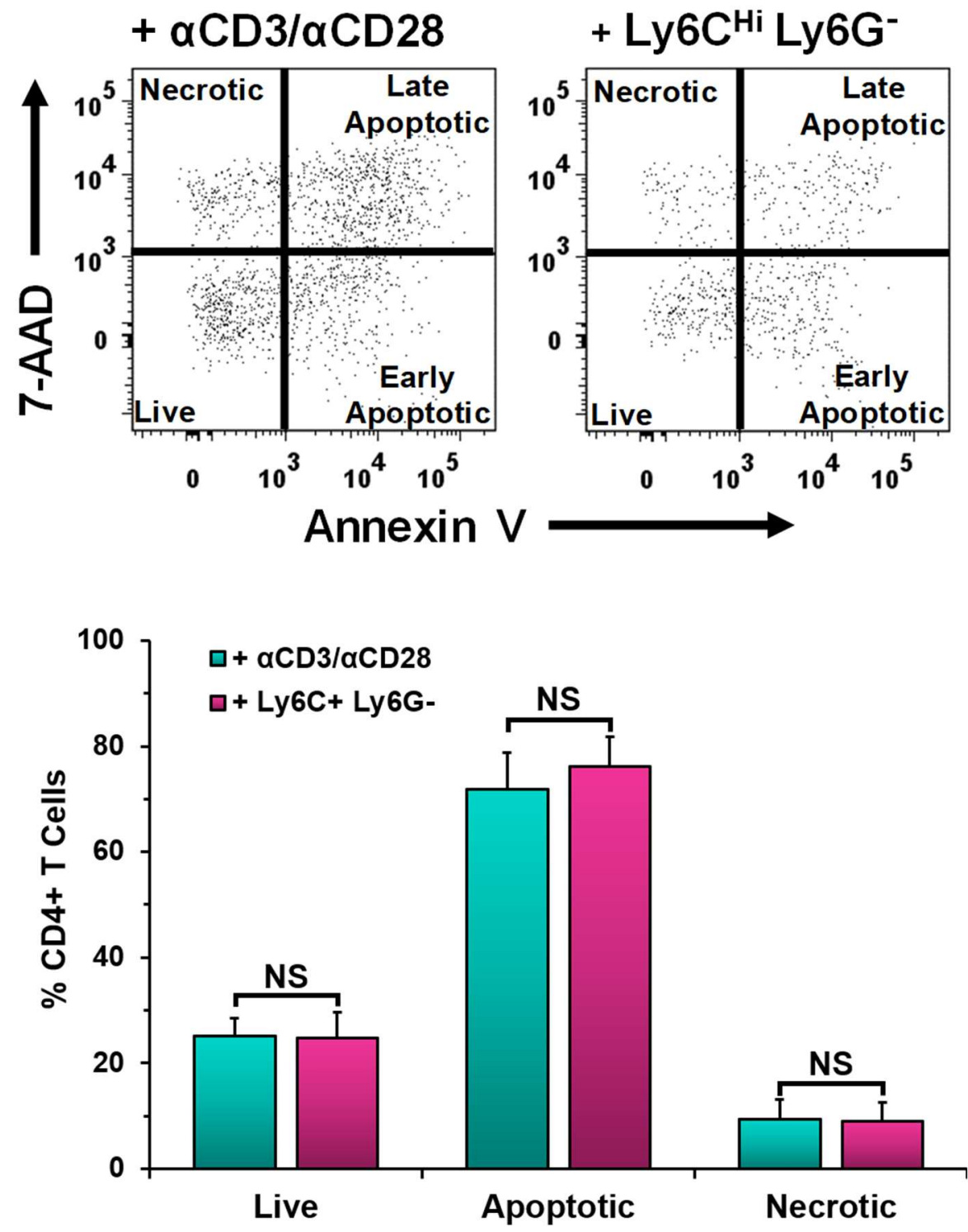

Figure 4.6: aMDSCs modulate T cell proliferation without inducing apoptosis. Naïve splenocytes were cultured by themselves, activated with plate-bound aCD3/aCD28, or activated and co-cultured with sorted aMDSCs. After five days splenocyte proliferation was assessed via MTT assay. (Upper) Representative dot plots depicting live, early apoptotic, late apoptotic, or necrotic $\mathrm{CD} 4^{+} \mathrm{T}$ cell subsets following 5-day culture of aCD3/aCD28-stimulated splenocytes alone (Left) or cocultured with aMDSCs (Right). (Lower) The proportion of live, apoptotic, or necrotic $\mathrm{CD}^{+}{ }^{+} \mathrm{T}$ cell subsets following 5 -day culture of $\alpha \mathrm{CD} 3 / \mathrm{\alpha CD} 28$-stimulated splenocytes alone (Blue) or co-cultured with sorted aMDSCs (Pink). Bars depict the mean percentage of $\mathrm{CD} 4^{+} \mathrm{T}$ cells that each subset makes up within that culture condition from two independent experiments where each co-culture was performed in triplicate, then combined for Annexin $V$ and 7-AAD staining and analysis. 


\section{Arginine Sequestration: aMDSC-mediated immune suppression is not dependent upon arginase activity.}

A common immunosuppressive mechanism used by MDSCs is arginine sequestration. Activated T cells require this essential amino acid to proliferate, but do not have the cellular machinery to synthesize it themselves, relying solely on they are able to import from their surroundings. Tumor-associated MDSCs interfere with this process by upregulating their expression of the argininemetabolizing enzymes arginase and iNOS, drastically reducing the bioavailability of this amino acid within the tumor microenvironment, arresting any further $\mathrm{T}$ cell proliferation [107].

We first examined the importance of arginase activity to aMDSC-mediated immune suppression. As before, we set up in vitro co-cultures of $\alpha \mathrm{CD} 3 / \alpha C D 28$ activated splenocytes with either the total SVF isolate, aMDSC-depleted ( $\triangle$ aMDSC) SVF, or FACS-isolated aMDSCs. To assess the role of arginase, one set of these co-cultures were resuspended in our normal culture media while we used media supplemented with the arginase inhibitor nor-NOHA $(200 \mu \mathrm{M})$ to resuspend the other set. After 5 days we found that blocking arginase activity did not affect the proliferative capacity of $\alpha \mathrm{CD} 3 / \mathrm{\alpha CD} 28$ activated splenocytes (Absorbance of $1.38 \pm 0.03$ vs $1.32 \pm 0.02$ at $450 \mathrm{~nm}$ ) (Figure 4.7). Additionally, it did not impair the immunomodulatory activity of aMDSCs, as the extent to which they suppressed splenocyte proliferation was relatively unchanged by the arginase blockade (Untreated vs nor-NOHA PI: $0.85 \pm 0.03$ vs $0.73 \pm 0.03$ ) (Figure 4.7). Indeed, save for a slight trend towards enhanced suppression in nor-NOHA treated $\triangle$ aMDSC SVF (Untreated vs nor-NOHA PI: $0.89 \pm 0.07$ vs $0.76 \pm 0.01)$ and 
aMDSC co-cultures, there was no clear difference between the untreated and norNOHA-treated wells for any of the co-culture conditions (Untreated vs nor-NOHA $\mathrm{Pl}: 1.2 \pm 0.4$ vs $1.1 \pm 0.08$ ) in terms of T cell proliferation (Figure 4.7), suggesting that the immunosuppressive abilities of aMDSCs, and of SVF cells in general, are likely not mediated by arginine sequestration. 


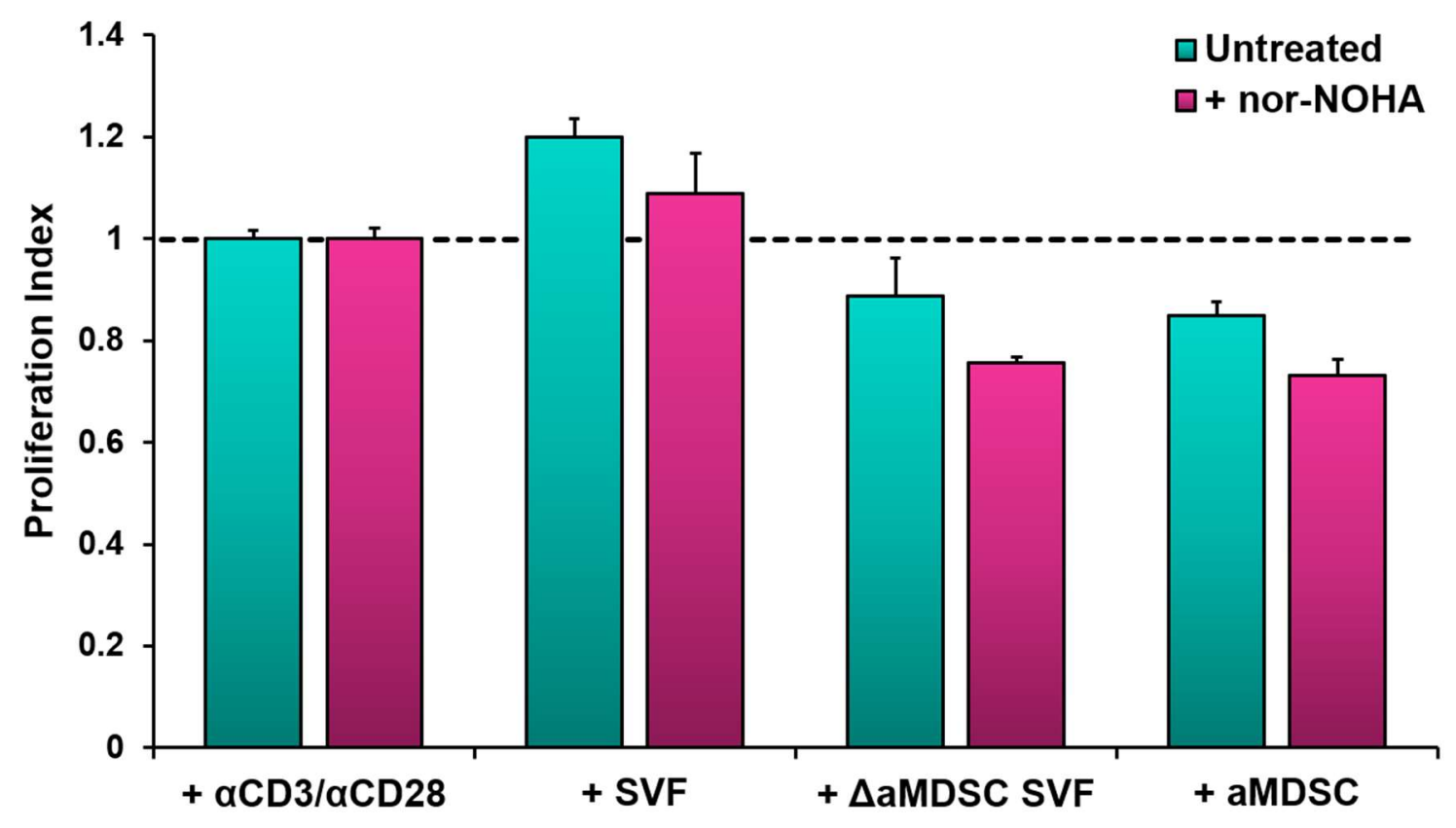

Figure 4.7: aMDSCs do not rely on arginase-mediated nutrient deprivation to suppress $T$ cell proliferation. Naïve splenocytes were cultured by themselves, activated with plate-bound $\alpha C D 3 / a C D 28$, or activated and co-cultured with various SVF subsets (Blue). nor-NOHA was added to some of the co-cultures to block arginase activity (Pink). After five days splenocyte proliferation was assessed via MTT assay. P.I. of untreated wells calculated relative to untreated $\alpha C D 3 / \alpha C D 28-$ stimulated splenocytes and P.I. of nor-NOHA co-cultures calculated relative to aCD3/aCD28-stimulated splenocytes cultured in media supplemented with norNOHA. Bars represent the mean P.I. in each well from one of four independent experiments where each culture condition was performed in triplicate and the error bars depict the SEM among those triplicate wells. 


\section{Immunosuppressive Cytokines: aMDSC cytokine production was below detection levels in vitro.}

We next analyzed the supernatants from these co-cultures using ELISAs to look for immunomodulatory cytokines that aMDSCs could be using to slow and eventually halt T cell proliferation altogether [100]. Specifically, we focused on IL10 and TGF $\beta$ as we found that aMDSCs were actively transcribing both cytokines in our characterization studies. In supernatants from one experiment we found, as one might expect, that IL-10 concentrations were higher in co-cultures with SVF (3.68 $\pm 0.44 \mathrm{pg} / \mathrm{mL}), \Delta$ aMDSC SVF $(2.01 \pm 0.98 \mathrm{pg} / \mathrm{mL})$, and sorted aMDSCs (1.96 $\pm 0.42 \mathrm{pg} / \mathrm{mL})$ than $\mathrm{aCD} 3 / \mathrm{aCD} 28$ activated splenocytes alone $(<0.00 \mathrm{pg} / \mathrm{mL})$

(Figure 4.8 a). However, these concentrations were at or below the assay's 5 $\mathrm{pg} / \mathrm{mL}$ limit of detection. Using supernatants collected from whole SVF co-cultures containing 6 - 7-fold more cells per well, we found a strong correlation between the supernatant concentration of IL-10 on culture day 2 and the number of SVF cells used to begin with (Figure $4.8 \mathrm{~b}$ ). As such, we were unable to use to measure IL-10 protein levels in the supernatants collected from our subsequent aMDSC coculture assays.

Fortunately, we were able to detect ample levels of TGF $\beta$ in these supernatants, finding it in every co-culture condition. This suggests including both that several different cell types are producing TGF $\beta$ in the SVF.

Intriguingly there was a marked increase in the concentration of TGF $\beta$ when arginase activity was blocked with nor-NOHA. This was one of the most consistent trends we observed across the supernatants we tested and was the most 
pronounced in the co-cultures with activated splenocytes and isolated aMDSCs $(113.24 \pm 8.6 \mathrm{pg} / \mathrm{mL}$ vs $173.6 \pm 14.0 \mathrm{pg} / \mathrm{mL})$ (Figure $4.9 \mathrm{a})$.

When we examined TGF $\beta$ concentrations in relation to the MTT proliferation results from the respective co-culture, we found a significant positive correlation (Pearson Coefficient $=0.7753)$. Surprisingly, it appeared as if $\mathrm{T}$ cell proliferation was at its peak in co-cultures with higher levels of TGF $\beta$ (Figure 4.9b, blue and pink markers). The implications of these findings are somewhat muddied however when you consider that whole splenocytes were used as the responding population inn these arginase blockade assays. Like the SVF, splenocytes are a heterogeneous mix of cells, which, in addition to T cells, contain various types of macrophages, dendritic cells, and B cells [156], any of whose function can be affected by interacting with MDSCs [89, 102, 107, 114]. It's possible that our aMDSCs are preventing TGF $\beta$ production in one or more of these splenic cells, causing the drop in TGF $\beta$ levels in the aMDSC and $\triangle$ aMDSC SVF co-cultures compared to our positive controls. Blocking arginase activity could partially restore the splenocytes' ability to produce this cytokine, explaining the increase we see in the nor-NOHA treated co-cultures.

If we focus on just aMDSC co-cultures (Figure $4.9 \mathrm{~b}$, orange markers), we see that, contrary to the overall trend, suppression increases (P.I. values decrease) as TGF $\beta$ levels rise. MDSCs are a very plastic cell type, capable of quickly adapting to shifts in their microenvironment. Therefore, it is possible that the aMDSCs in our co-cultures upregulated their production of TGF $\beta$ in response 
to the arginase blockade, allowing them to continue to suppress activated $\mathrm{T}$ cell proliferation. 


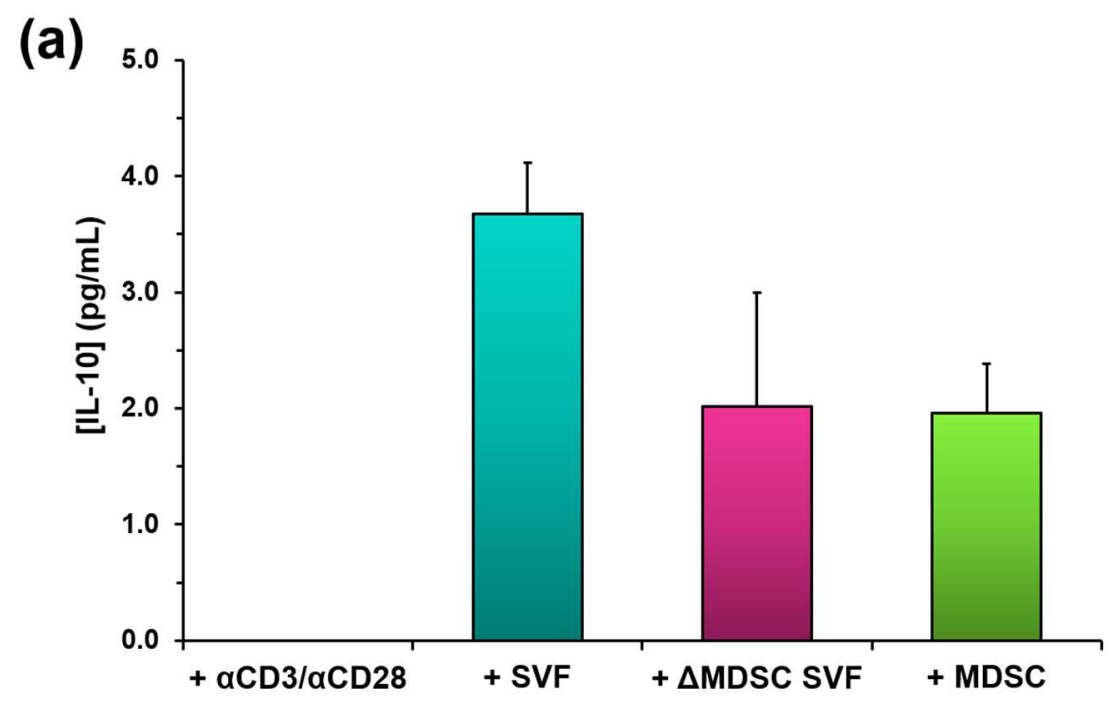

(b)

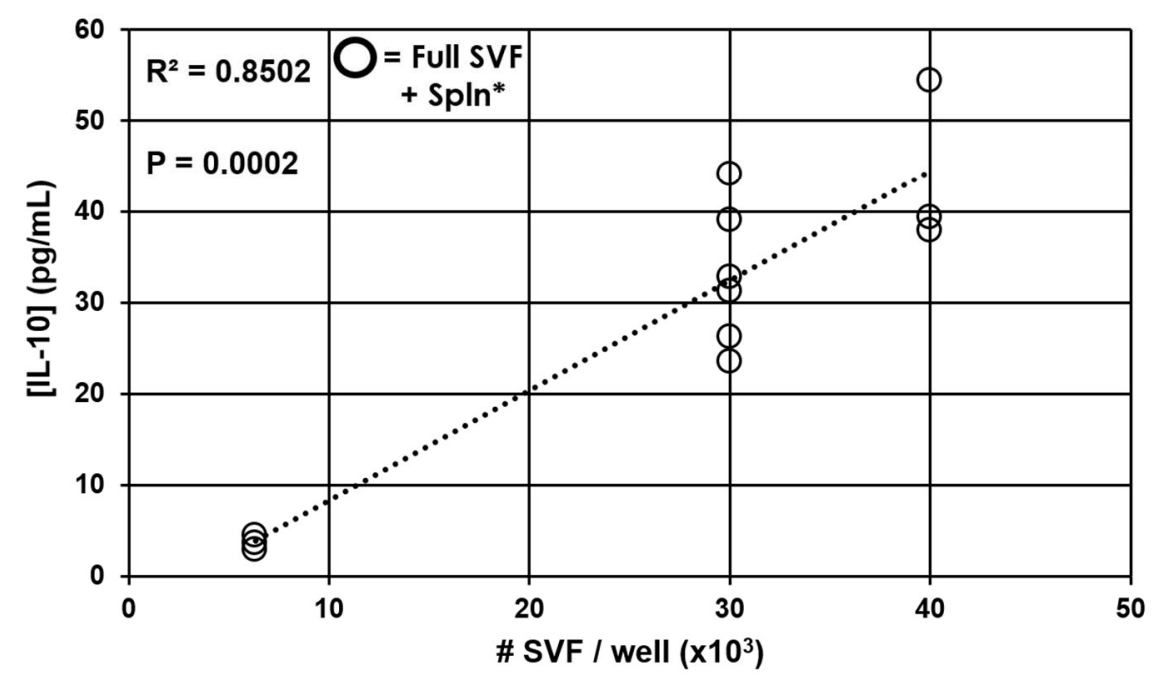

Figure 4.8: IL-10 concentrations were below detection in aMDSC co-cultures due to small number of cells in each well. Naïve splenocytes were cultured by themselves, activated with plate-bound $\alpha C D 3 / \alpha C D 28$, or activated and co-cultured with various SVF subsets. Supernatants were collected from each well after two days and stored at $-80^{\circ} \mathrm{C}$ for later analysis. (a) Mean IL-10 concentrations as determined by ELISA. Data derived from the supernatants of one suppression assay where each condition was performed in triplicate. The error bars depict the SEM among those triplicate wells. (b) Correlation plot of IL-10 concentrations in Full SVF co-cultures vs the number of SVF cells plated in each well on day 0 . Data derived from the supernatants of three independent experiments where SVF was co-cultured with aCD3/aCD28-stimulated splenocytes. Each marker represents one well. $R^{2}$ value derived from linear regression. Spln*: $\alpha C D 3 / \alpha C D 28$-stimulated splenocytes. $P$ value obtained using Pearson coefficient. 
(a)

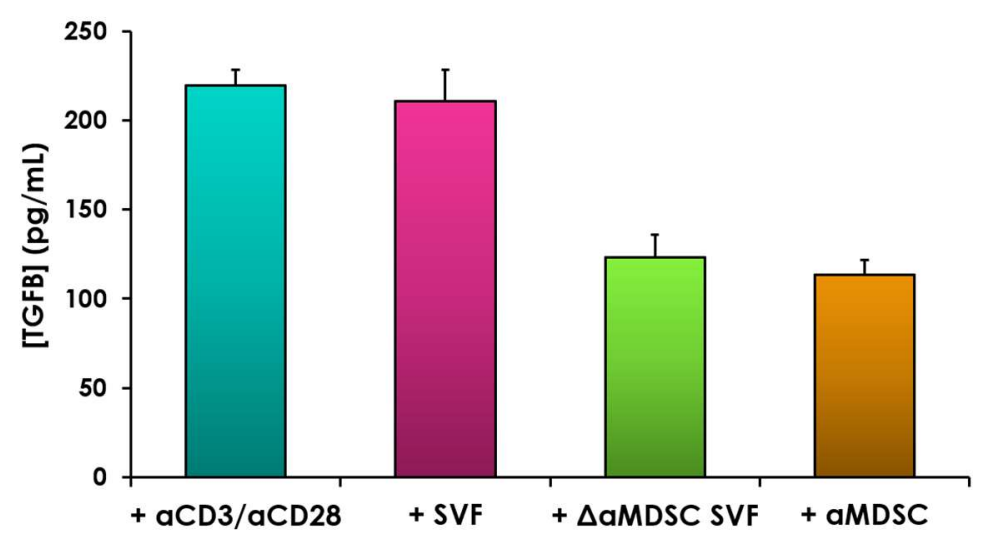

(b)
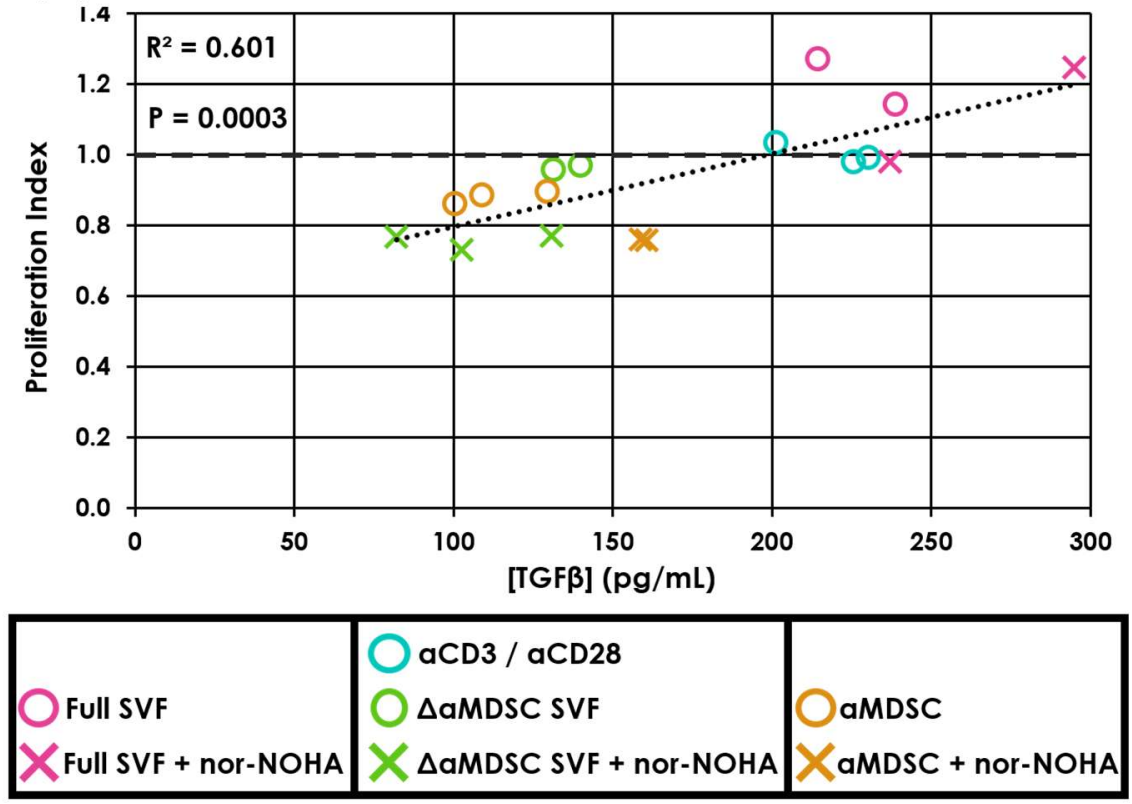

Figure 4.9: aMDSCs may modulate immune cell activity via TGF $\beta$ production. Naïve splenocytes were cultured by themselves, activated with plate-bound aCD3/aCD28, or activated and co-cultured with various SVF subsets. Supernatants were collected from each well after two days and stored at $-80^{\circ} \mathrm{C}$ for later analysis. (a) Mean concentration of TGF $\beta$ as determined by ELISA. Bars represent the mean protein concentration from of three independent suppression assays where each culture condition was performed in triplicate and error bars depict the SEM among those triplicate wells. (b) Correlation plot of proliferation index vs TGF $\beta$ concentration. Each co-culture condition is represented by a different color. Circles denote wells where cells were cultured in normal media and $X$ 's represent wells where the media was supplemented with nor-NOHA. $R^{2}$ value derived from linear regression. $P$ value obtained using Pearson coefficient. 


\section{Conclusions}

According to the nomenclature standards recommended by Bronte et al (2016) (ref. 22), a population of cells must fulfill three key requirements in order to be classified as MDSCs. First, the myeloid cell population must increase in numbers in a pathological setting as compared to healthy conditions. Second, these cells should possess a characteristic combination of phenotypic markers, which includes surface protein, biochemical, and molecular markers. Finally, and most importantly, they need to be able to suppress inflammatory immune responses. Our adipose-derived cells are CD11 $b^{+}$CD301- CD11c- Ly6C HilLow Ly6G ${ }^{+/}$, do not express macrophage markers such as F4/80 and CD301, express canonical MDSC-associated genes, significantly attenuate activated $\mathrm{T}$ cell proliferation, and are known to accumulate in the adipose tissue in obesity [129]. Taking all of this into account we conclude that these cells represent a bona fide population of tissue resident MDSCs within the AT of lean individuals (aMDSCs).

As for the effector mechanisms behind the immunomodulatory nature of these aMDSCs, we can only speculate. Based on our in vitro data, we know that aMDSCs abrogate $T$ cell proliferation without preventing their initial activation and proliferation. They also do not promote apoptosis in these proliferating T cells. This is consistent with other reports documenting their ability to induce senescence in activated $\mathrm{T}$ cells or regulate their proliferation using methods such as nutrient sequestration, cytokine production, and nitrosylation [102, 157]. We performed cursory investigations into each but were unable to definitively identify any one of them as the primary suppressive method. We believe that, similar to what others 
have shown, these adipose-resident MDSCs most likely employ a combination of techniques in order to stem T cell proliferation [102]. 


\section{CHAPTER 5: MYELOID DERIVED SUPPRESSOR CELLS REGULATE T CELL NUMBERS \& ADIPOSE MACROPHAGE POLARIZATION IN HEALTHY ADIPOSE TISSUE}

Once we determined that these cells were bona fide MDSCs, we immediately were curious as to what role they were playing in healthy adipose tissue, particularly since these cells are typically not found in homeostatic immune states. Ideally, to investigate this we would specifically delete MDSCs from the AT in vivo and monitor any downstream effects. Unfortunately, the heterogeneous nature of MDSCs makes it very difficult to develop either an inducible deletion or transgenic knockout model. To date the best method available to remove MDSCs in vivo is through the periodic administration of the $\alpha \mathrm{Gr} 1$ antibody. This monoclonal antibody successfully removes all Ly6G positive and a portion of the Ly6C positive cells from circulation no matter whether it is delivered intraperitoneally, subcutaneously, or intravascularly. We chose to specifically look for changes to the $\mathrm{T}$ cell and adipose resident macrophage populations as the phenotypes and functions of their tumor-associated counterparts are greatly influenced by MDSCs $[98,158,159]$.

\section{Gr-1 Model of MDSC Depletion}

Since I was ultimately interested in how adipose-resident MDSCs (aMDSCs) fit into the established immune cell network of healthy tissue, I needed 
to develop a way to see how the tissue was affected when these cells were taken away. Currently, there is no MDSC-specific knock-out mouse model, but there is a well-established, antibody-mediated model for in vivo MDSC depletion, which utilizes the RB6-8C5 clone of the aGr1 antibody. Historically, this model was first used as a means to investigate neutrophil function in vivo $[160,161]$ but has since been adopted by MDSC researchers as a method to study their impact in various pathological settings [130, 162-165]. In addition to MDSCs and neutrophils, the Gr1 protein is also expressed by subsets of monocytes [162], dendritic cells [166], and even $\mathrm{CD}^{+} \mathrm{T}$ cells [167], all of which are susceptible to aGr1-mediated depletion. To further complicate matters, the actual depletion efficiency of this model varies between different tissue types [168, 169], with additional questions even having been raised regarding the standard methods used to verify successful depletion $[168,170]$.

With no other effective method for MDSC depletion commercially available we decided to go ahead with this model as it would allow us to compare the data from our experiments with the plethora of data from previously published studies. Additionally, we hypothesized that any effects we observed following the $\alpha \mathrm{Gr} 1$ treatment would have directly resulted from the loss of aMDSCs in this tissue and not from the depletion of these other cells $[154,171,172]$, since we would be using adipose from healthy, non-obese mice for the initial phase of our study and healthy adipose contains very few neutrophils or CD8 ${ }^{+} \mathrm{T}$ cells (Figure 5.1).

Moreover, where we could, we tried to minimize and control for the influence of this model's inherent "messiness". For instance, the RB6-8C5 Gr1 antibody 
clone, used for depletion sterically hinders the binding of most Ly6G and $\mathrm{Gr} 1$ antibodies available commercially, which means that neither of these markers can be used in conjunction with flow cytometry or fluorescent microscopy to validate successful MDSC depletion. As such, we made sure to take this into account when we designed the flow cytometry staining panels for the depletion experiments.

As mentioned above, there is evidence that this antibody is not efficient at removing MDSCs from all tissues [169]. To determine whether this model would remove aMDSCs from visceral adipose depots, we i.p. injected 4 mice (2 males and 2 females) with $200 \mu \mathrm{g}$ of the aGr1 antibody every 3-4 days for two weeks. The adipose tissue from these mice was pooled for analysis while their spleens were analyzed individually to verify successful MDSC depletion. Initially it appeared as if the antibody had not penetrated into the fat pads of these mice as there was no real difference in the proportion of aMDSCs in aGr1-treated and untreated SVF. However, individual analysis of splenocytes from each of these mice revealed that the depletion had only been effective in two out of the four animals, potentially explaining the results of our SVF analysis (Figure 5.2). There were two main differences between the group of mice where the treatment worked and those mice where it didn't. The primary factor delineating the two groups was sex; the aGr1 treatment had successfully removed $\mathrm{Ly}_{6 \mathrm{C}^{+}} \mathrm{Ly} 6 \mathrm{G}^{-}$and $\mathrm{Ly} 6 \mathrm{C}^{+} \mathrm{Ly}_{6 \mathrm{G}} \mathrm{G}^{+}$cells from the spleens of the female mice, while these two populations were still present in the male spleens. In addition to sex, the female mice weighed significantly less than the males, implicating weight as another potential factor involved in determining whether or not the antibody treatment would be effective. Since sexually dimorphic 
differences in antibody-mediated depletion had not been described in the literature we concluded that weight was more likely to be the determining factor behind the variations in our treatment efficacy.

With no other MDSC depletion model available we decided to address these issues on several fronts. First, we changed the dosing and administration protocols to account for weight differences between animals, as well as differences is the size and location of the visceral fat pads. We exchanged the standard $200 \mu \mathrm{g} / \mathrm{mouse}$ dose for one that was based on the weight of each mouse $(15 \mu \mathrm{g} / \mathrm{g})$. In this way we could account for size difference between individuals.

Additionally, we altered our administration route, moving from intraperitoneal (i.p.) to subcutaneous (s.c.) injections in order to address concerns regarding differences in fat pad location and size between males and females. The SVF cells we harvest in our studies are isolated from the epididymal fat pads in males and the parametrial fat pads in females. Although these two pet depots serve physiologically analogous functions, anatomical differences mean that the fat pads themselves are more superficial in males than those in the females. Their location puts them directly in the area targeted in i.p. injections. Since we were interested in the treatment's impact on specifically this tissue, we wanted to avoid any potential complications or confounding results that could arise from accidentally injecting the antibody directly into the fat pad. We found that s.c. administration was just as effective as i.p. injection and improved the consistency of the depletion between animals. 
As a final means of optimization, we decided to improve the "resolution" of our flow cytometric SVF analyses by modifying our cell isolation protocol. Up until this point the vast majority of SVF experiments in our lab were performed to investigate its efficacy as a novel cell-based therapeutic. Since the cellular composition of the AT immune compartment can be affected by factors such as age [173], sex [174], weight, stress level [175, 176], and even vendor, there can be quite a bit of variation among SVF isolates from one healthy animal to the next. Using co-housed mice can greatly reduce this variability (Figure $\mathbf{5 . 3}$ a), but this was not always possible for experiments that require a large number of SVF cells. To avoid potential downstream consequences of this variability on experimental outcomes, the lab developed a protocol that isolated SVF cells from the adipose of $4-10$ mice that was pooled. Each aspect of this isolation protocol had been rigorously and meticulously optimized to maximize the final cell yield and produce a consistent, standardized cell isolate. Combining tissues from multiple animals yielded a homogeneous cell product that reflected the "average" proportion and distribution of each cell type found in the SVF, effectively normalizing the composition of the final SVF isolate by erasing the variations observed among individual mice.

Although ideal for functional assays, the normalizing influence of pooling tissues from multiple mice was masking some of the more subtle changes that took place on an individual level as we observed in our pilot depletion experiments (Figure 5.3 a). If we wanted to capture the subtle changes that may result from removing such a small population of cells from the AT, we would need to improve 
the sensitivity of our analyses, so I developed a version of our isolation protocol that could reliably isolate SVF cells from the visceral fat pads of a single mouse. The new, downsized protocol would allow us to examine MDSC depletion-induced changes in AT immune cell dynamics that would have otherwise been masked by the homogenization inherent to working with pooled tissue samples. Two major modifications to this procedure included using smaller collection vessels and minimizing excess plasticware. For example, in the pooled isolation process, the adipose tissue is minced and homogenized in a non-tissue culture treated $100 \mathrm{~mm}$ petri dish. The homogenate is then transferred to a $50 \mathrm{~mL}$ conical tube for collagenase digestion. If this is done is a timely fashion, issues such as losing macrophages to plastic adherence are kept to a minimum. However, for the individual isolation we would be working with far fewer cells, so we needed to eliminate as many steps as possible from the process where cells were transferred from one container to another. For the homogenization step this meant mincing the whole tissue in the same tube where it would be digested.

In general we tried to keep the actual collagenase digestion process as consistent with the pooled isolation protocol as possible, using the same volume of (1 $\mathrm{mL} / \mathrm{mL}$ AT) and recipe for (2 $\mathrm{mg}$ collagenase / $\mathrm{mL} \mathrm{AT;} 1 \mathrm{mg}$ DNase / $\mathrm{mL}$ AT; and $1 \mathrm{~mL} 0.1 \%$ BSA in DCF-PBS / $\mathrm{mL}$ AT) the digestion buffer. Additionally, we used the same digestion time of 35 minutes for these individual SVF isolations. We did find it necessary however to modify the environmental conditions in which the digestion took place. In a standard SVF isolation, we use a programmable, climate-controlled rotator (Enviro-Genie; Scientific Industries Inc, Bohemia, NY) to 
control the exact temperature and rotation speed of the tubes during the collagenase digestion. However, the smaller $5 \mathrm{~mL}$ tubes used in the individual SVF isolation protocol did not fit in this machine. As an alternative, we digested the homogenized adipose samples inside of a cell culture incubator to maintain the $37^{\circ} \mathrm{C}$ temperature required for maximum enzyme efficacy. This change in digestion environment ultimately introduced two major differences between the individual and pooled SVF isolation protocols: $\mathrm{CO}_{2}$ levels and rotation speed. First, the Enviro-Genie is not airtight, so pooled tissues are digested at environmental $\mathrm{CO}_{2}$ levels $\left(0.035 \%-.1 \% \mathrm{CO}_{2}\right)$, while the cell culture incubator maintains $\mathrm{CO}_{2}$ levels at around $5 \%$. To minimize the potential for any confounding effects of this difference on the digestion process, the tubes containing the tissue and digestion buffer were closed and sealed with parafilm in environmental $\mathrm{CO}_{2}$ conditions before being placed into the incubator. Switching from the Environ-Genie to the incubator presented an additional challenge when it came to proper agitation of the tissues during digestion. Previous work in the lab had found that got optimal results from the collagenase digestions at a rotation speed of $35 \mathrm{rpm}$. Unfortunately, digesting the tissues in the incubator created a relatively large disparity in the rotation speed between the two protocols as the generic blood rotator used to agitate the samples had a fixed speed of $18 \mathrm{rpm}$. These two factors did not seem to influence the efficiency of the collagenase enzyme itself as the tissues from both protocols appeared to be completely digested after 35 minutes.

Even with these modifications in place, we were able to procure SVF samples from individual mice that were comparable in composition to what we 
produced with the pooled isolation protocol (Figure 5.3 b). Ultimately, by improving our SVF isolation methods, optimizing the $\alpha \mathrm{Gr} 1$ dose for each mouse, and changing how the antibody was delivered, we were able to successfully deplete MDSCs from both the spleens and adipose tissue of aGr1-treated mice. 


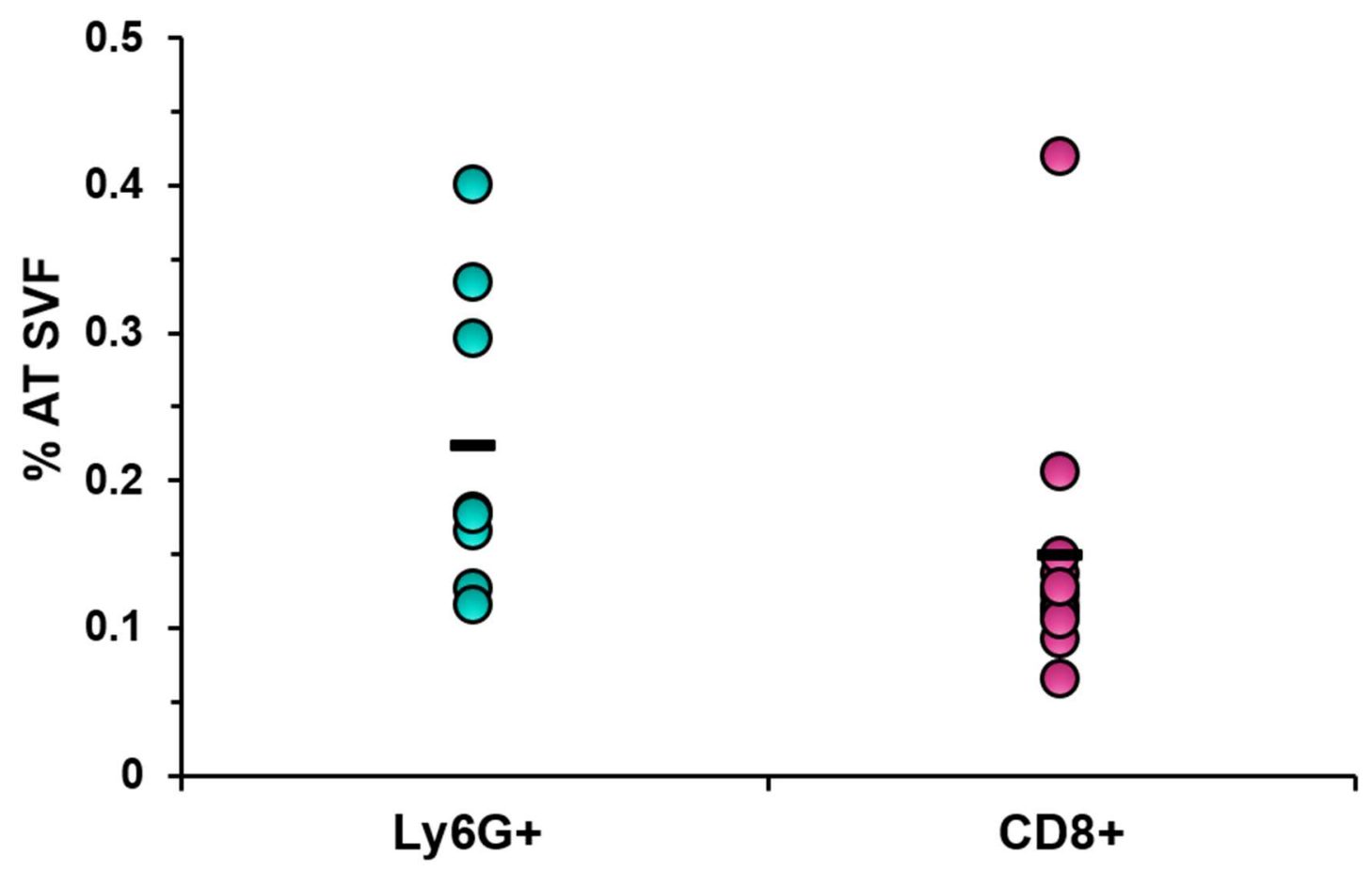

Figure 5.1: Healthy adipose tissue contains very few of the non-MDSC cells affected by treatment with $\alpha \mathrm{Gr} 1$. Frequency of $\mathrm{CD} 11 \mathrm{~b}^{+} \mathrm{Ly}_{6 \mathrm{G}^{+}}\left(\mathrm{Ly} 6 \mathrm{G}^{+}\right)$cells and $\mathrm{CD}^{+} \mathrm{T}$ cells in the stromal vascular cell fraction (SVF) of healthy AT. Each circle represents one of eleven healthy mice that were analyzed individually. 

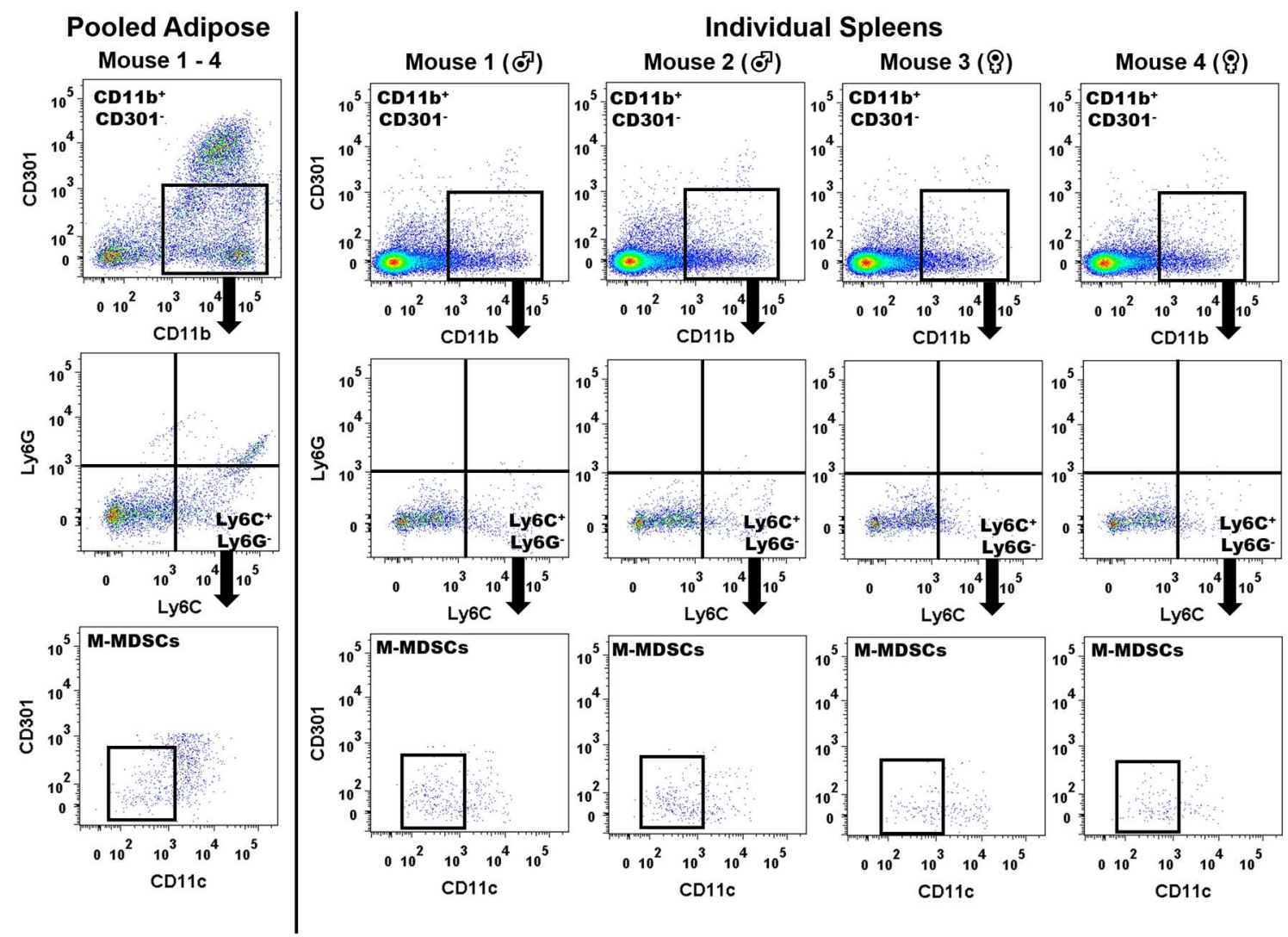

Figure 5.2: Pooling adipose tissue for analysis masks differences in treatment efficacy between individual animals. Healthy, $16-20$-week old

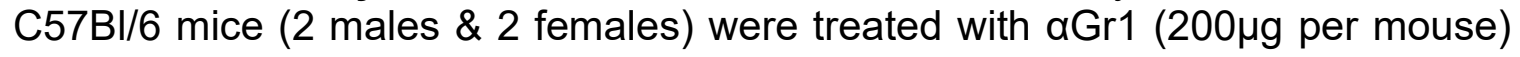
every $3-4$ days for 1 week. Tissues were harvested and stained for flow cytometric analysis. Gonadal fat pads were pooled for analysis (Far Left Column) while spleens from each mouse were analyzed individually to verify successful MDSC depletion. Dot plots depict the gating strategy used to characterize MMDSCs in each tissue. 
a
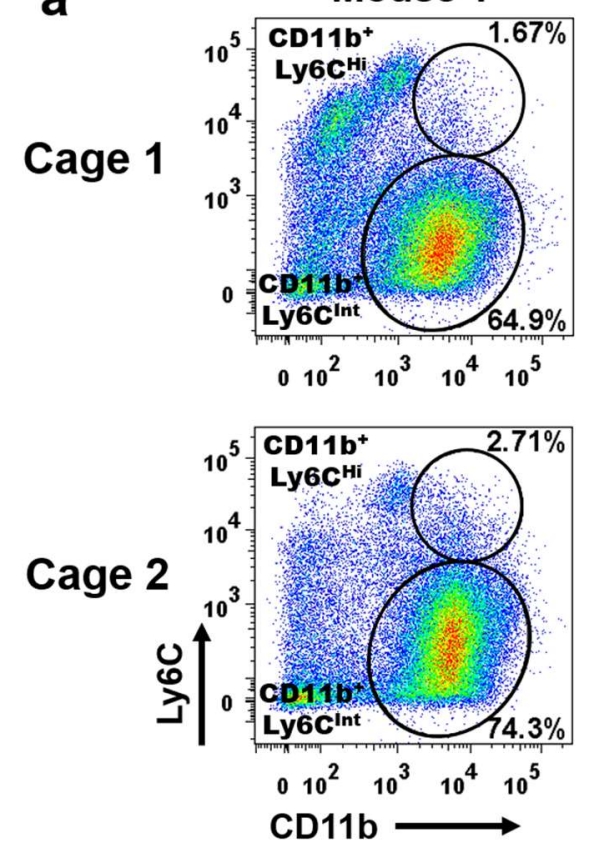

b

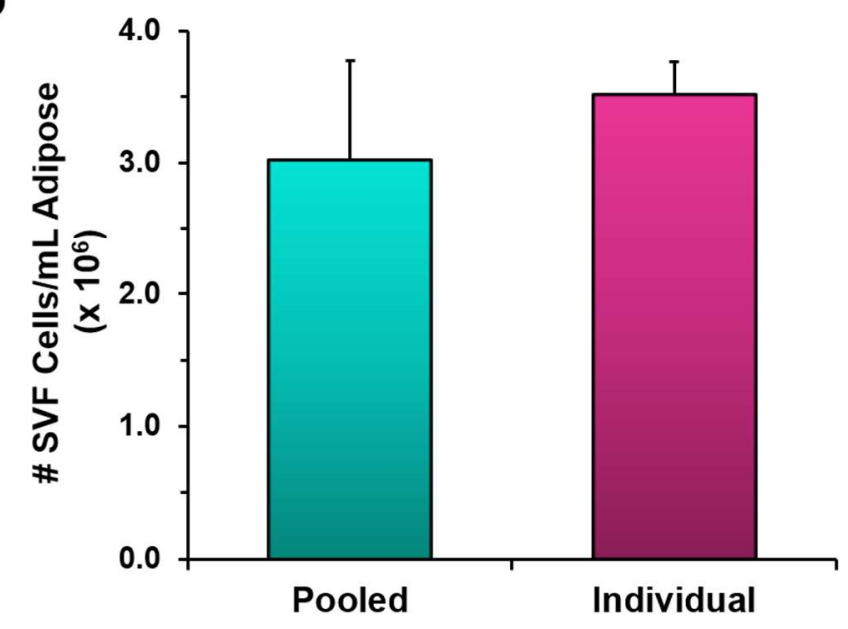

Mouse 2
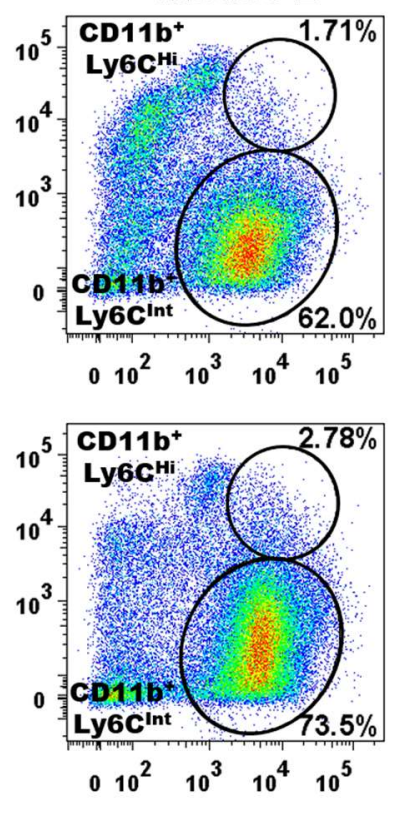

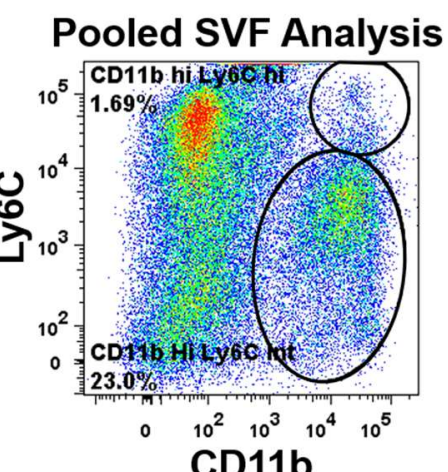

CD11b

Figure 5.3: Analyzing AT SVF from individuals reveals cage-dependent variations in immune cell population distribution. Healthy, $16-20$-week old C57BI/6 mice were treated with aGr1 $(15 \mu \mathrm{g} / \mathrm{g})$ every $3-4$ days for 1 week. Epididymal fat pads were collected one day after the final antibody injection. (a) Dot plots comparing myeloid populations in AT SVF from four aGr1-treated mice raised and housed in two separate cages. Values displayed in each plot are percentage of $\mathrm{CD}_{4} 5^{+}$cells that the specified population represents. (b) The absolute number of SVF cells obtained (normalized to the volume of adipose tissue harvested) from either pooled (Blue) or individual (Pink) SVF isolation protocols. Bars depict the mean of 6 pooled or 19 individual, representative SVF isolations performed independently. Error bars denote the SEM. 


\section{aGr1 effectively removes cells from spleens \& adipose after 1 week of treatment.}

To the best of our knowledge, this finding represents the first time that immunosuppressive MDSCs have been identified in otherwise healthy adipose tissue. To better understand the role these adipose-resident MDSCs may play in metabolically healthy tissue, we employed an antibody-mediated model of in vivo MDSC depletion. Mice received subcutaneous injections of the $\alpha \mathrm{Gr} 1$ antibody $(15 \mu \mathrm{g} / \mathrm{g})$ every 3-4 days for one or four weeks. This range of time points allowed us to assess both the acute and chronic effects of the $\alpha \mathrm{Gr} 1$ treatment in the AT. One day after their final dose, the epididymal fat pads were collected from each mouse and examined individually for any differences in the distribution of the various resident immune cell populations using flow cytometry. After 1 week, splenocyte analysis confirmed that the treatment had effectively depleted CD11 $\mathrm{b}^{+}$ CD11C- Ly6C HilLow Ly6G $^{+/-}$cells systemically, with the number of these cells dropping from $78.0 \pm 9.6 \times 10^{4}$ cells/spleen to $5.4 \pm 0.9 \times 10^{4}$ cells/spleen (Figure $5.4 \mathbf{a}-\mathbf{b})$. Following treatment, there was a significant reduction in both subsets of adipose-resident of MDSCs (aMDSCs), with a 79.4\% decrease in the absolute number of Ly6G-M-MDSCs $\left(15.8 \times 10^{3} \pm 2.4\right.$ cells $/ \mathrm{mL}$ AT vs $3.3 \times 10^{3} \pm 1.3$ cells $/ \mathrm{mL} \mathrm{AT})$ and a $95.3 \%$ reduction in Ly6G ${ }^{+}$PMN-MDSC numbers $\left(3.0 \times 10^{3} \pm\right.$ 0.98 cells $/ \mathrm{mL}$ AT vs $0.14 \times 10^{3} \pm 0.06$ cells $/ \mathrm{mL}$ AT) (Figure $5.5 \mathrm{a}-\mathbf{b}$ ). To determine if the changes we observed in the adipose following the $\alpha \mathrm{Gr} 1$ treatment were nonspecific effects of the antibody treatment, we treated a subset of our mice with rat IgG2b isotype antibody (15 $\mathrm{\mu g} / \mathrm{g}$ ) every 3-4 days for 1 week. There was no difference in the proportion of CD11 $b^{+}$CD301- Ly6C HilLow Ly6G $^{+/}$cells AT SVF 
cells between the isotype-treated $(0.8 \pm 0.1 \%)$ and untreated $(0.77 \pm 0.07 \%$ of the total SVF) animals (Figure 5.6).

Unexpectedly, we found that the efficiency of aGr1-mediated MDSC depletion lessened over time. After 4 weeks of $a \mathrm{Gr} 1$ treatment, CD11 $b^{+} \mathrm{CD} 11 \mathrm{c}^{-}$ Ly6C HilLow Ly6G $^{+-}$cells represented $0.6 \pm 0.08 \%$ of splenocytes $(31.85 \pm 7.03$ cells/spleen $)$ and $0.31 \pm 0.06 \%$ of the total SVF isolate $(0.95 \pm 0.19$ cells $/ \mathrm{mL}$ AT $)$ (Figure 5.4 b \& 5.5 b). Although inconvenient, this resurgence of CD11 $\mathrm{b}^{+}$ Ly6C HilLow Ly6G $^{+/-}$cells is not unprecedented. A 2016 study by Moses et al [170] describes a "Ly-6G+ cell rebound phase" after regular treatment (twice weekly) with the $a \mathrm{Gr} 1$ antibody for more than 10 days. They found that immature, CD11 $\mathrm{b}^{+}$ $\mathrm{Ly}_{6 \mathrm{G}}{ }^{+}$accumulated in the spleens and circulation of mice that received twice weekly $a \mathrm{Gr} 1$ treatments for more than one week. They attributed this effect to elevated rates of myelopoiesis caused by increased pressure on the bone marrow to replenish the $\alpha \mathrm{Gr} 1-$ depleted cell populations. Gene transcription and flow cytometric characterization of these "rebounded" cells revealed that they likely served an immunomodulatory function in vivo. Additionally, they refer to, but do not show, data directly demonstrating their ability to suppress activated $\mathrm{T}$ cell proliferation. For our part, we were unable to perform functional characterization studies on the CD11 $b^{+}$Ly6C $^{\text {Hillow }}$ Ly6G $^{+/-}$cells we found in 4 week $a \mathrm{Gr} 1$-treated mice since we could not feasibly isolate a sufficient number of these cells from the SVF of chronically treated animals to set up viable T cell proliferation/suppression assays. However, given their phenotypic similarity to those cells described by Moses et al, it is likely that the CD11 b $\mathrm{Ly}^{+ \text {HilLow }} \mathrm{Ly}_{6 G^{+/-}}$SVF cells we observed 
after 4 weeks aGr1 treatment represents a partial restoration of the immunomodulatory AT-resident MDSCs.

Since the RB6-8C5 clone of the Gr1 antibody has also been used to deplete neutrophils [160], we assessed the proportion and absolute number of CD11 $\mathrm{b}^{+}$ Ly6G ${ }^{+}$cells in healthy adipose tissue before and after the aGr1 treatment. As stated in the previous chapter, we found that $C D 11 b^{+} L y 6 G^{+}$cells only accounted for about $0.15 \pm 0.03 \%$ of the total SVF $\left(3.72 \times 10^{3} \pm 0.77\right.$ cells $/ \mathrm{mL}$ AT $)$, with the majority being Ly6C Low Ly6G+ PMN-MDSCs (Figure 4.2). Following the aGr1 treatment the percentage of CD11 $b^{+} \mathrm{Ly}_{6 G^{+}}$cells in the total SVF dropped to 0.04 $\pm 0.02 \%\left(0.99 \times 10^{3} \pm 0.28\right.$ cells / mL AT) (Figure 5.7). Given this relative paucity of $\mathrm{Ly}_{6 \mathrm{G}}{ }^{+}$cells in the adipose tissue of healthy mice, we concluded that any aGr1 treatment-induced responses in the adipose tissue were most likely directly related to the loss of the tissue-resident MDSCs. 
(a)
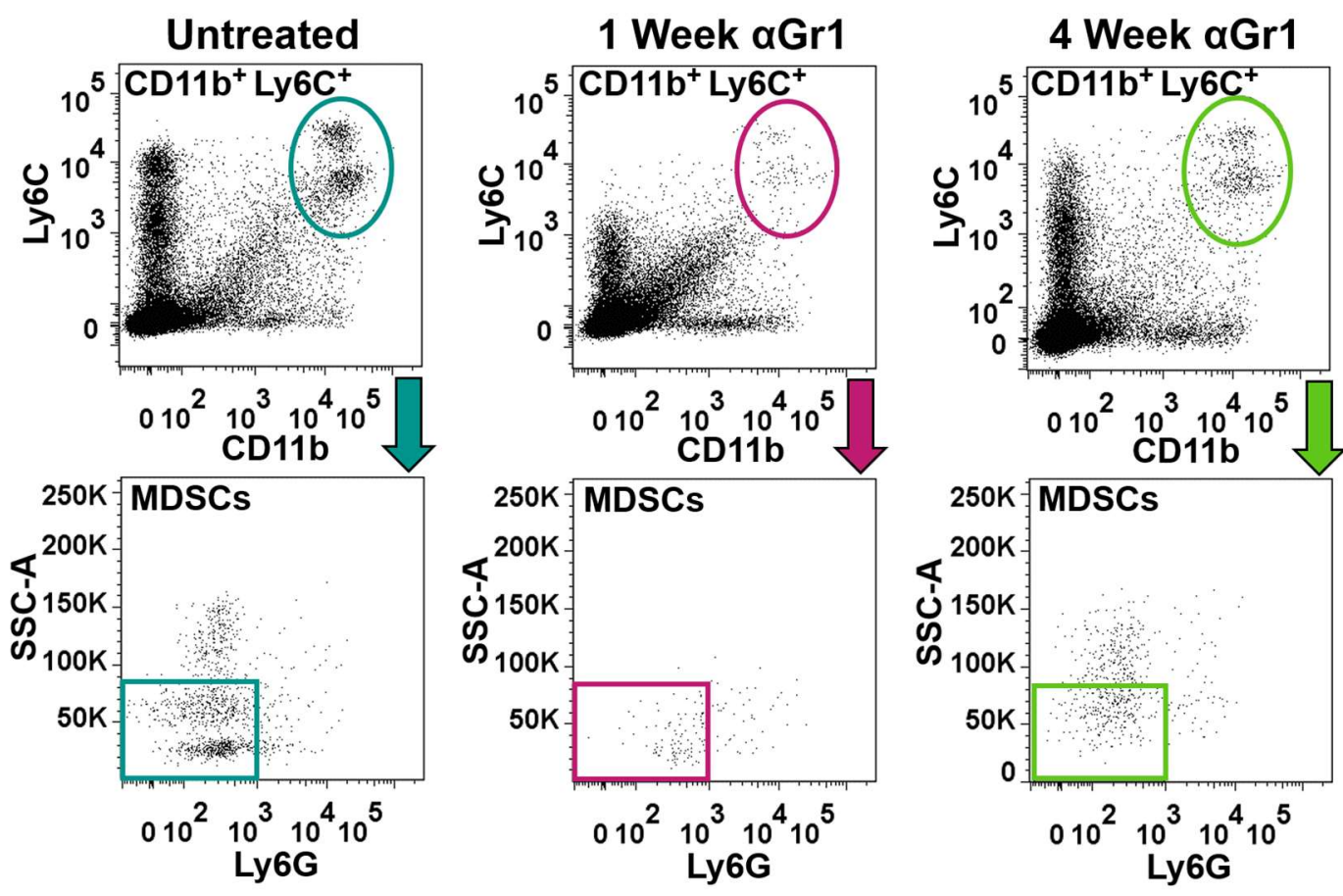

(b)

Ly6G
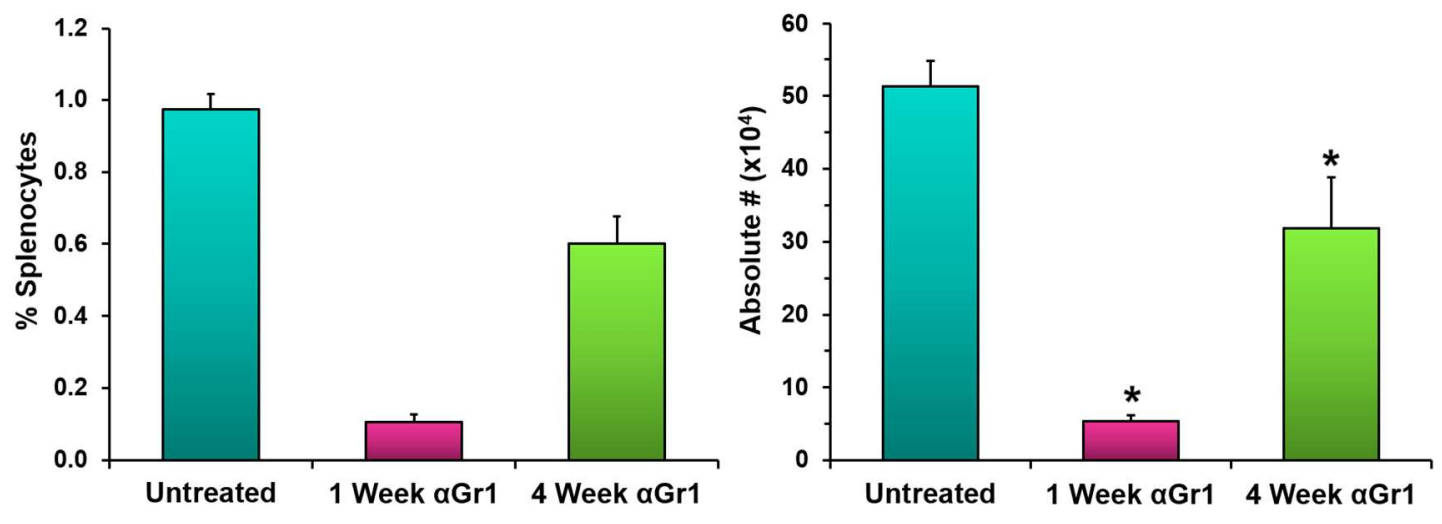

Figure 5.4: $\alpha \mathrm{Gr} 1$ antibody treatment efficiently depletes splenic MDSCs. Healthy, 16 - 20-week old C57BI/6 mice were treated with $\alpha \mathrm{Gr} 1(15 \mu \mathrm{g} / \mathrm{g})$ every 3 -4 days for 1 or 4 weeks. Spleens were collected one day after the final antibody injection. (a) Representative dot plots of splenocytes from healthy, aGr1-treated or untreated mice. Circle gates indicate cell populations targeted by the aGr1 antibody. Selected dot plots for each treatment group derived from one animal, representative of the mean of their respective groups. (b) The frequency (Left) and absolute number (Right) of splenic MDSCs (Ly6C ${ }^{H i}$ Ly6G- M-MDSCs + Ly6C Low Ly6G ${ }^{+}$PMN-MDSCs) in untreated (Blue) mice or animals receiving 1- (Pink) or 4weeks of $\alpha \mathrm{Gr} 1$ treatment (Green) mice. Bars depict the mean of 11 untreated, 81 week $\alpha \mathrm{Gr} 1$-treated, and 6 4-week $\alpha \mathrm{Gr} 1$-treated mice. Differences between $\alpha \mathrm{Gr} 1$ treated and untreated animals determined using a Student's $t$-test. *: $P<0.05$. 
(a)

Untreated
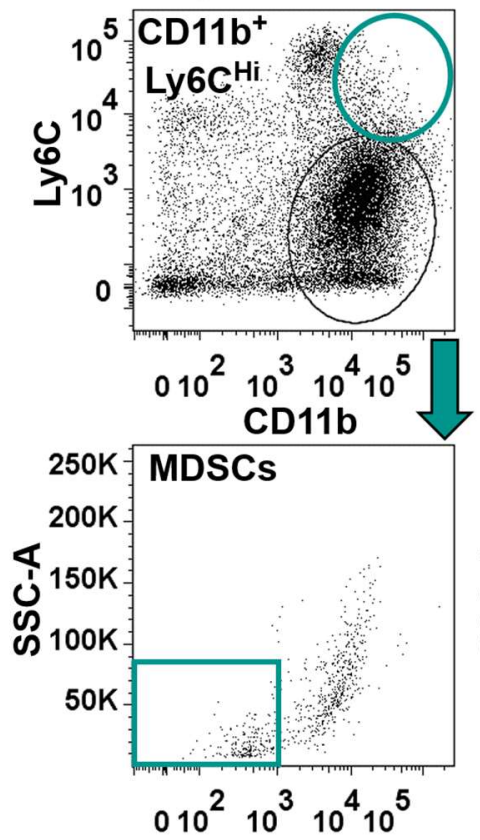

Ly6G
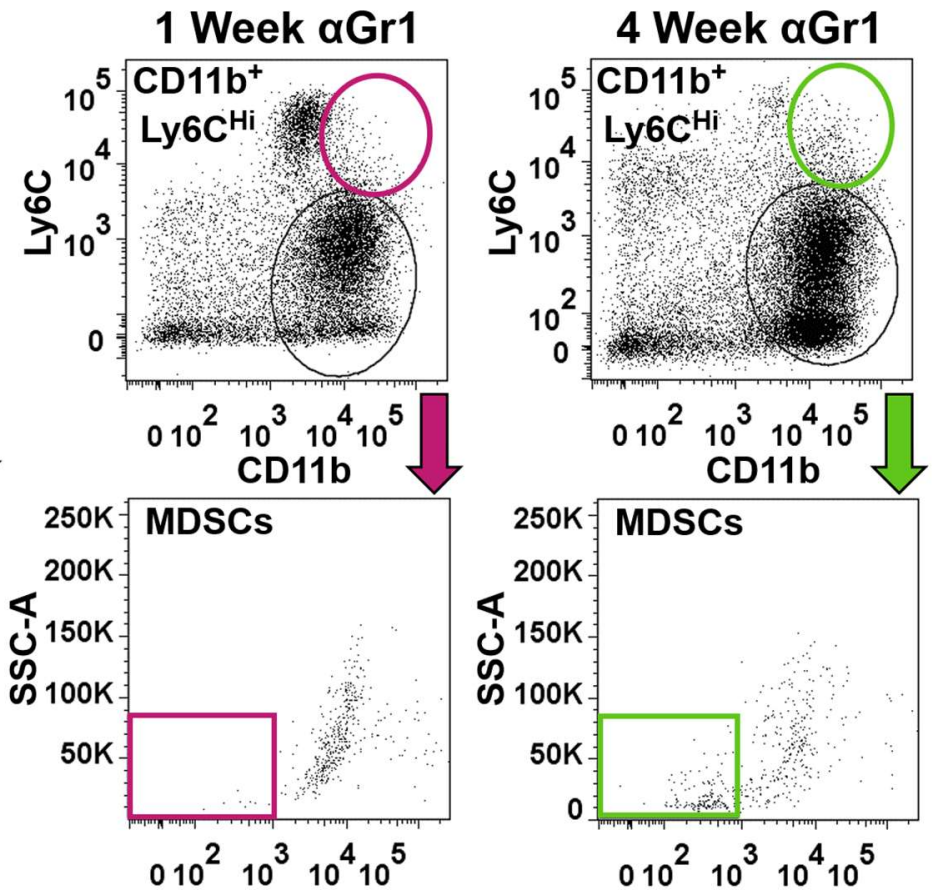

Ly6G

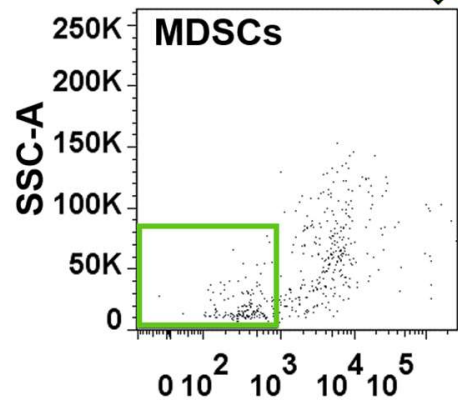

Ly6G

(b)
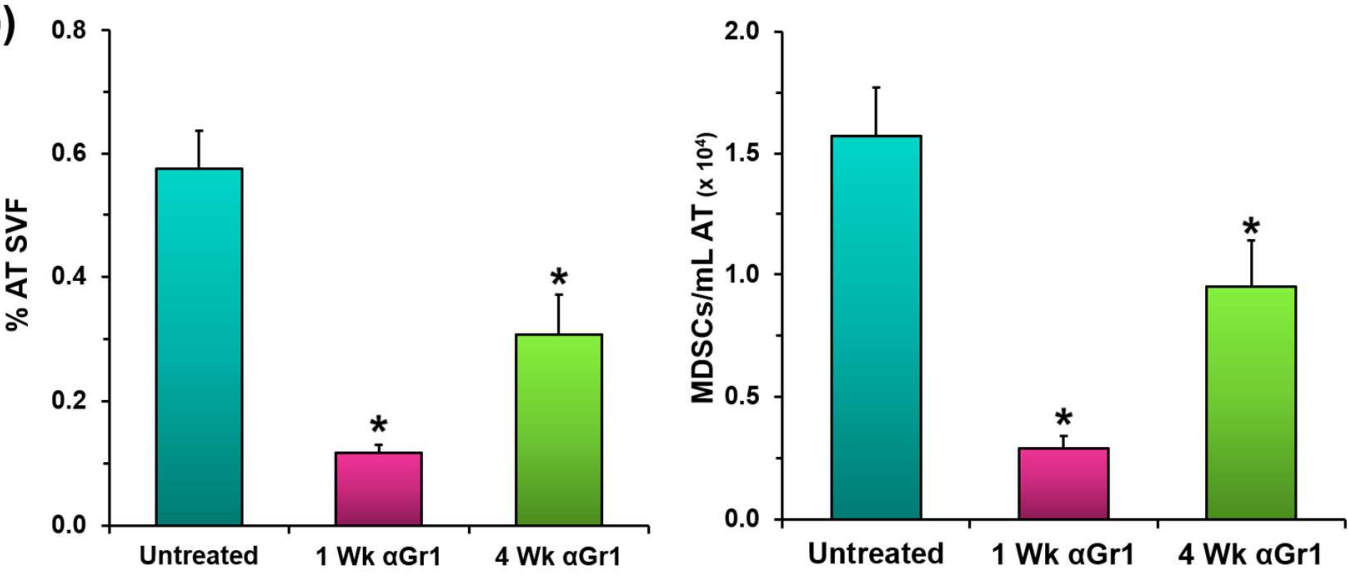

Figure 5.5: $\alpha \mathrm{Gr} 1$ antibody treatment efficiently removes MDSCs from epididymal fat pads. Healthy, $16-20$-week old $\mathrm{C} 57 \mathrm{BI} / 6$ mice were treated with aGr1 $(15 \mu \mathrm{g} / \mathrm{g})$ every $3-4$ days for 1 or 4 weeks. Epididymal fat pads were collected one day after the final antibody injection. (a) Representative dot plots of CD11 $\mathrm{b}^{+}$F4/80- adipose tissue (AT) stromal vascular fraction cells (SVF) from healthy, aGr1-treated or untreated mice. Circle gates indicate cells targeted by the aGr1 antibody. Selected dot plots for each treatment group derived from one animal, representative of the mean of their respective groups. (b) The frequency (Left) and absolute number (Right) of aMDSCs (M-MDSCs + PMN-MDSCs) in AT SVF from untreated (Blue) mice or animals receiving 1-week (Pink) or 4-weeks of aGr1 treatment (Green). Bars depict the mean of 11 untreated, 81 -week $a \mathrm{Gr} 1$ treated, or 64 -week $\alpha \mathrm{Gr} 1$-treated mice. Differences between $\alpha \mathrm{Gr} 1$-treated and untreated animals determined using a Student's $t$-test. *: $P<0.05$. 
(a)
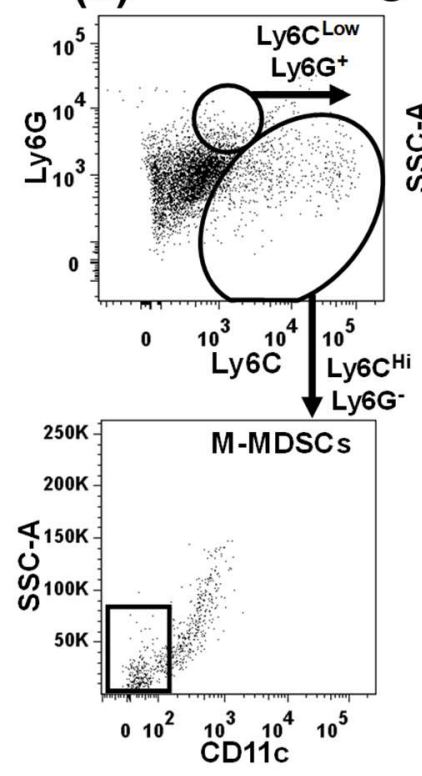

$\lg \mathbf{2} \mathbf{b}$

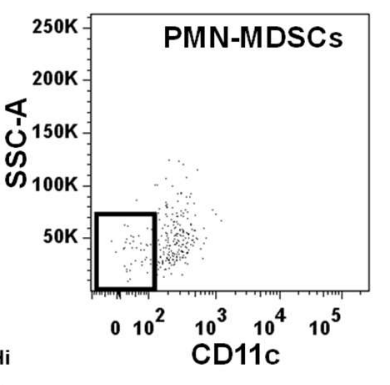

(b)
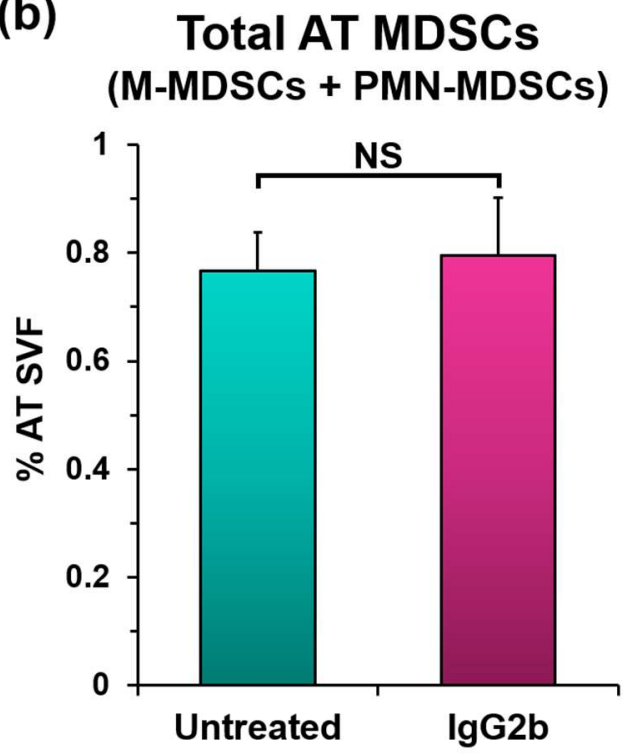

Figure 5.6: Generic antibody treatment does not affect the adipose-resident MDSC population. Healthy, $16-20$-week old C57BI/6 mice were treated with IgG2b antibody $(15 \mu \mathrm{g} / \mathrm{g})$ every $3-4$ days for 1 week. Epididymal fat pads and spleens were collected one day after the final antibody injection. (a) Representative dot plots of CD11 $b^{+}$F4/80- SVF cells IgG2b-treated mice. Selected dot plot derived from one animal and is representative of the group's mean. (b) The frequency of aMDSCs (M-MDSCs + PMN-MDSCs) in AT SVF from untreated (Blue) and IgG2b-treated mice (Pink). Bars depict the mean of 15 untreated or 5 lgG2b-treated mice from five independent experiments. Differences between treated and untreated animals determined using a Student's $t$-test. NS: P > 0.05 . 

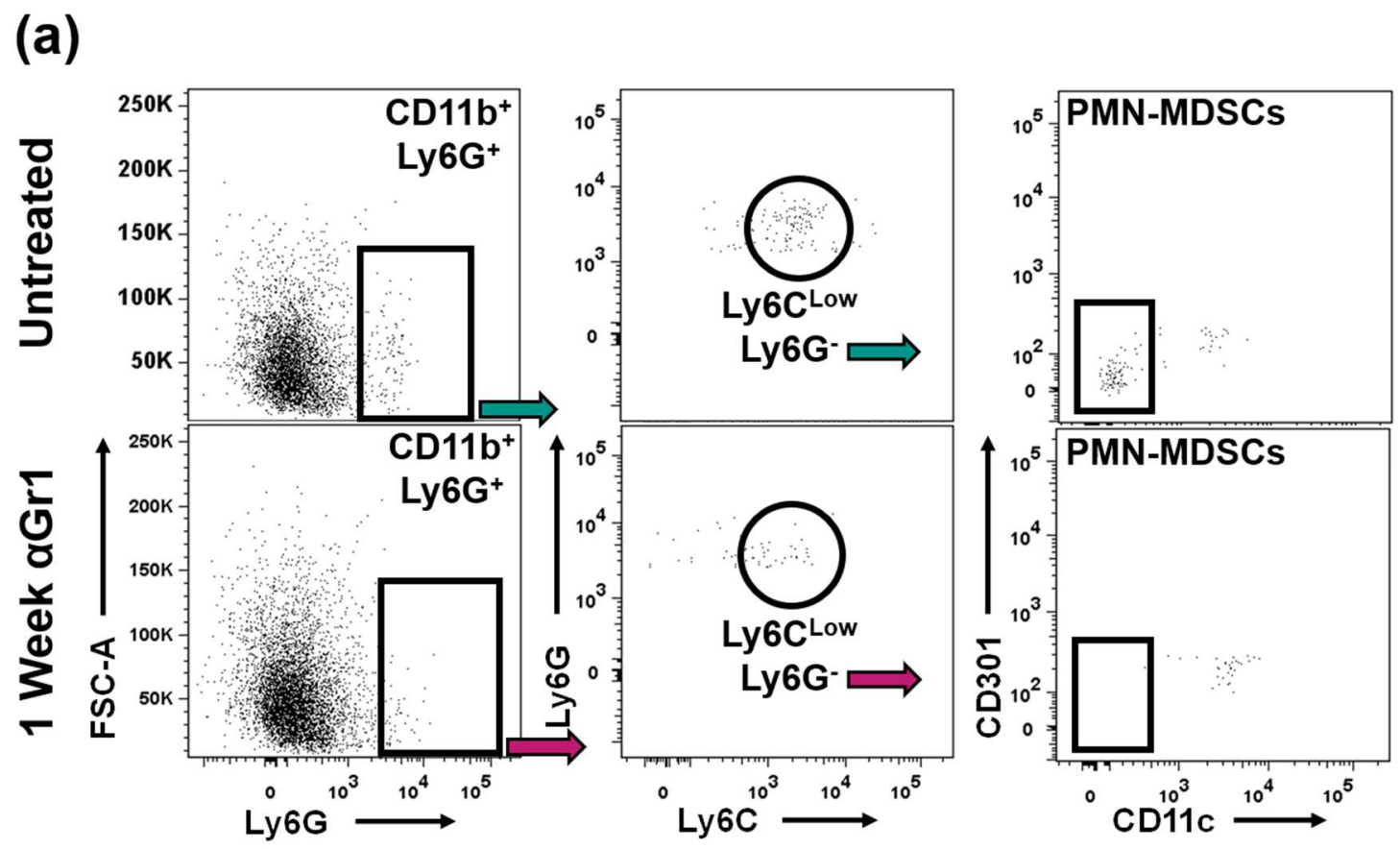

(b)
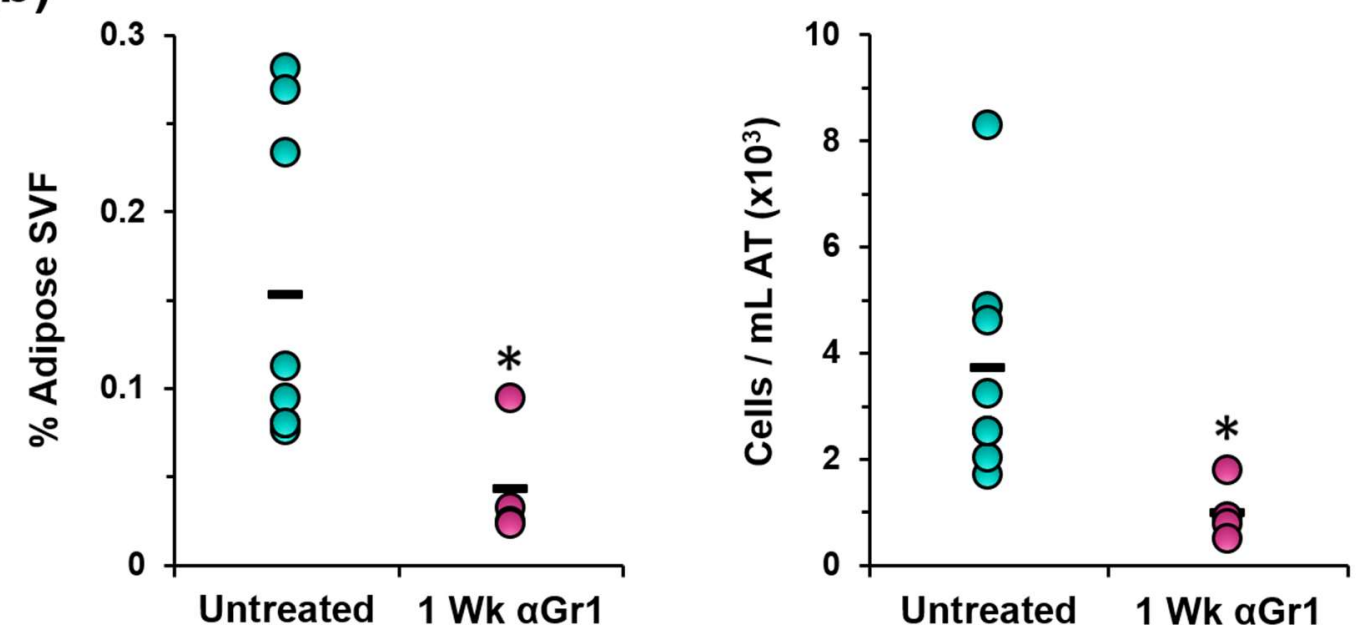

Figure 5.7: Ly6G+ SVF cells are efficiently depleted following treatment with aGr1 antibody. (a) Dot plots characterizing $\mathrm{CD} 11 \mathrm{~b}^{+} \mathrm{Ly} \mathrm{G}^{+}$cell subsets in untreated (Upper) and aGr1-treated (Lower) mice. (b) The frequency (Left) and absolute number (Right) of $\mathrm{Ly} 6 \mathrm{G}^{+}$cells in the SVF of untreated (Blue) and aGr1treated (Pink) mice. Horizontal bars represent the mean of 8 untreated or 4 aGr1treated animals from two independent experiments. Differences between the $\alpha$ Gr1-treated and untreated animals determined using a Student's $t$-test. *: P < 0.05 . 


\section{Adiposity increases when MDSCs are removed from adipose tissue.}

Although we did not collect extensive metabolic data on our $\alpha \mathrm{Gr} 1$ treated animals, we did weigh them after each injection and weighed their fat pads after tissue harvest. These two measures allowed us to calculate a crude percent adiposity for each mouse at the end of the experiments. Ultimately, we found that the $\alpha \mathrm{Gr} 1$ treatment did not affect the overall weight of the mice, even after 4 weeks of regular injections (Figure 5.8 a).

Compared to untreated mice, acute $\alpha \mathrm{Gr} 1$ treatment significantly increased adiposity; however, this effect disappears in our 4 week treated mice (Figure 5.8 b). Without any more detailed metabolic data it is difficult to draw any clear conclusions from these findings. Particularly since factors unrelated to our treatment, such as environmental changes, altered microbiota, or where they came from (in-house colony vs purchased from a vendor), could influence the animal's metabolism and lipid burden. Moreover, there was no change in the number of SVF cells per milliliter of adipose tissue which indicates that the treatment was not inducing any overt or drastic changes in the tissue's overall function at any point (Figure $5.8 \mathrm{c}$ ). Intriguingly, we did find a moderate negative correlation between the percent adiposity of an individual mouse and the number of aMDSCs found in their adipose, suggesting that aMDSCs may play a role in lipid uptake by adipocytes (Figure $5.8 \mathrm{~d}$ ). 
(a)

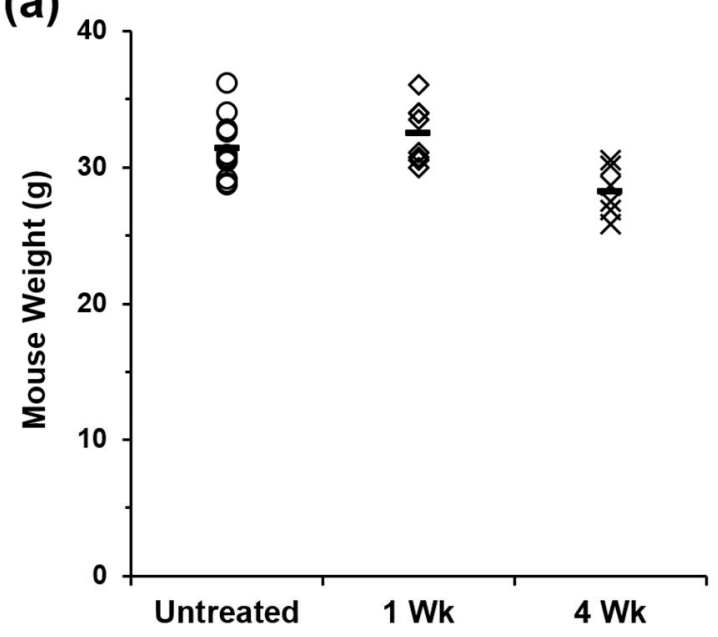

(c)

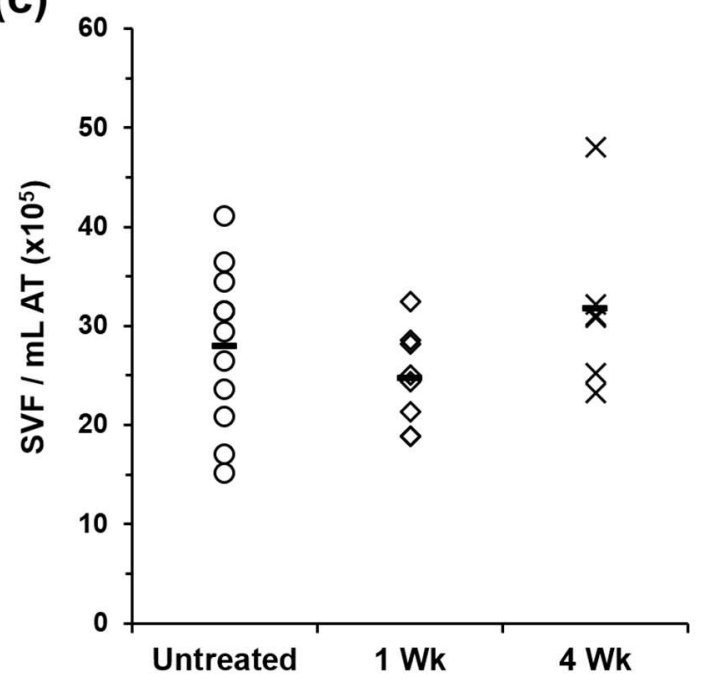

(b)

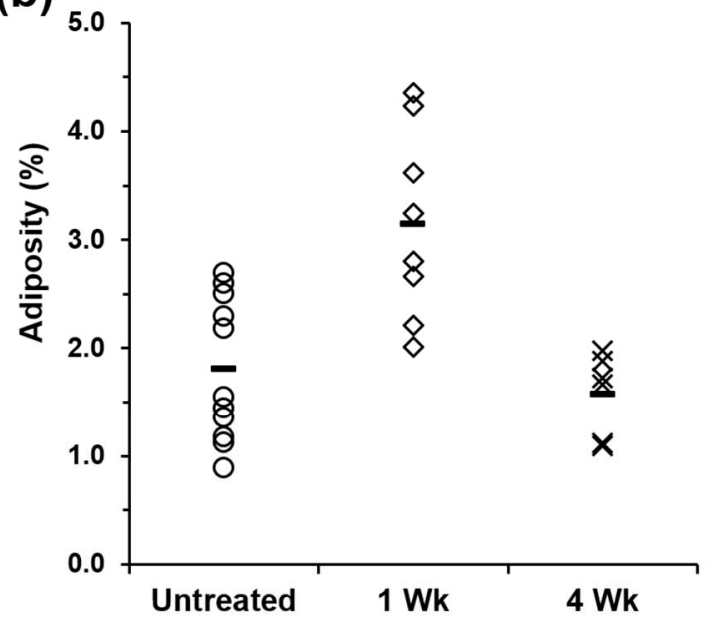

(d)

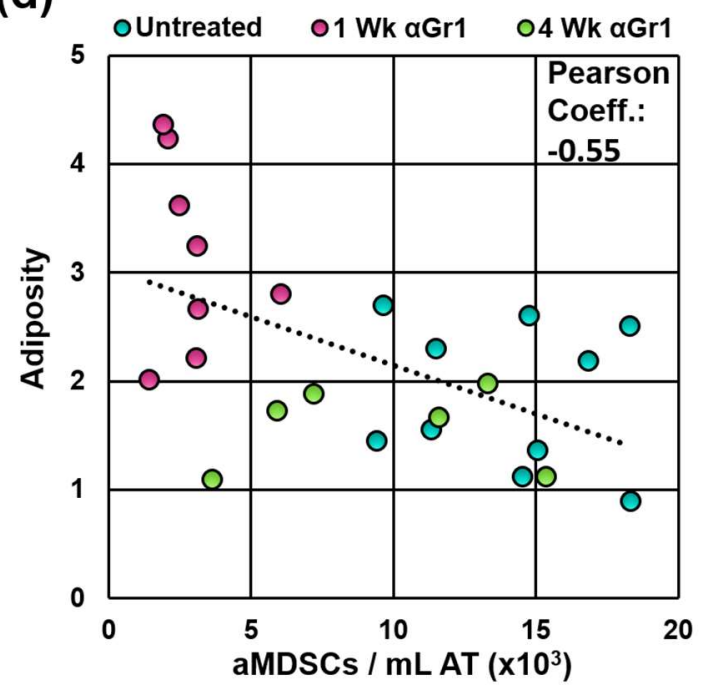

Figure 5.8: aMDSC depletion does not affect superficial measures of metabolic health or the cellularity of adipose tissue. Healthy, $16-20$-week old C57BI/6 mice were treated with $\alpha \mathrm{Gr} 1(15 \mu \mathrm{g} / \mathrm{g})$ every $3-4$ days for 1 or 4 weeks. Epididymal fat pads and spleens were collected one day after the final antibody injection. (a) Weight, (b) percent adiposity [(fat pad weight / total weight) $\times 100 \%$ ], (c) and the absolute number of SVF cells / $\mathrm{mL}$ adipose tissue in untreated (Blue), 1-week (Pink), or 4-week (Green) aGr1-treated mice. (d) Absolute number of aMDSCs / mL AT vs percent adiposity. Each circle represents an individual mouse from the indicated treatment group. Pearson Coeff.: Pearson Coefficient (derived from linear regression. Data pooled from 4 independent experiments. 


\section{Both acute \& chronic MDSC depletion increases the number of $T$ cells in healthy adipose tissue.}

In general, the absolute number and proportions of the various AT-resident immune cell subsets were unaffected by MDSC depletion, with one notable exception: there was a significant accumulation of T cells in the adipose of $\alpha \mathrm{Gr}-1$ treated mice (Figure 5.9 a). CD4 ${ }^{+}$T cells make up around $0.8 \pm 0.07 \%$ of the SVF isolate $\left(1.9 \pm 0.14 \times 10^{4}\right.$ cells $\left./ \mathrm{mL} \mathrm{AT}\right)$ in healthy, $16-20$-week old male mice. The frequency of these cells increased to $1.43 \pm 0.3 \%$ of the total SVF $\left(3.44 \pm 0.6 \mathrm{CD} 4^{+}\right.$ $\mathrm{T}$ cells $/ \mathrm{mL}$ AT) after only 1 week of the $a \mathrm{Gr} 1$ treatment, expanding further to 1.85 $\pm 0.05 \%$ of the SVF $\left(5.28 \pm 0.4 \mathrm{CD}^{+}{ }^{+} \mathrm{T}\right.$ cells $/ \mathrm{mL}$ AT $)$ after 4 weeks of bi weekly antibody injections (Figure 5.9 b). Treatment with the $\lg$ G2b antibody isotype did not affect the CD4 ${ }^{+}$SVF population, confirming that their accumulation was directly related to the loss of adipose-resident MDSCs and was not an off-target effect of the antibody treatment itself (Figure $5.10 \mathrm{a}-\mathbf{b}$ ).

Additionally, there was no change in splenic $\mathrm{CD}^{+}{ }^{+} \mathrm{T}$ cell number $(5.01 \pm 0.6$ $\times 10^{6} \mathrm{CD}^{+} \mathrm{T}$ cells/spleen vs $5.32 \pm 0.5 \times 10^{6} \mathrm{CD}^{+} \mathrm{T}$ cells/spleen) or frequency (9.55 $\pm 0.5 \%$ vs $10.17 \pm 0.8 \%)$ between 1 week-treated and untreated controls, indicating that the influx of $\mathrm{CD}^{+} \mathrm{T}$ cells was isolated to the adipose tissue at this early time point (Figure $5.10 \mathrm{c}$ ). After 4 weeks of the $\alpha \mathrm{Gr} 1$ treatment however, the percentage $(17.5 \pm 1.5 \%)$ and absolute number $\left(9.07 \pm 1.4 \times 10^{6} \mathrm{CD}^{+} \mathrm{T}\right.$ cells/spleen) of splenic $\mathrm{CD}^{+}{ }^{+} \mathrm{T}$ cells significantly increases, suggesting that prolonged MDSC depletion may lead to low grade inflammation systemically.

Compared to untreated controls, both short-term and long-term aGr1 treatment significantly reduced the frequency $(6.42 \pm 0.6 \%$ vs $2.9 \pm 0.1 \%$ vs 2.16 
$\pm .0 .2 \%)$ and absolute numbers $\left(3.27 \pm 0.2 \times 10^{6} \mathrm{CD}^{+} \mathrm{T}\right.$ cells/spleen vs $1.51 \pm 0.1$ $\times 10^{6} \mathrm{CD}^{+} \mathrm{T}$ cells/spleen vs $1.13 \pm 0.2 \times 10^{6} \mathrm{CD}^{+} \mathrm{T}$ cells/spleen) of splenic CD8+ $\mathrm{T}$ cells (Figure $\mathbf{5 . 1 0} \mathrm{d}$ ). This was likely due to the in vivo $\mathrm{T}$ cell-eliminating properties of the RB6-8C5 clone of the Gr1 antibody [167]. This depleting effect did not impact the levels of $\mathrm{CD} 8^{+}$cells in the adipose tissue however, as there was not change in their frequency $(0.16 \pm 0.04 \%$ vs $0.19 \pm 0.04 \%$ of the SVF) and number $\left(0.5 \pm 0.1 \times 10^{4}\right.$ cells $/ \mathrm{mL}$ AT vs $0.49 \pm 0.04 \times 10^{4}$ cells $/ \mathrm{mL}$ AT $)$ of these cells following short-term MDSC depletion (Figure $5.9 \mathrm{c}$ ). Moreover, SVF from mice that had undergone chronic MDSC depletion had significantly more $\mathrm{CD} 8^{+} \mathrm{T}$ cells $\left(1.5 \pm 0.2 \%\right.$ of the SVF \& $4.6 \pm 0.5 \times 10^{4} \mathrm{CD}^{+} \mathrm{T}$ cells $\left./ \mathrm{mL} \mathrm{AT}\right)$ compared to their untreated and 1 week treated counter parts (Figure $5.9 \mathrm{c}$ ). The Gr1-mediated ablation of splenic $\mathrm{CD} 8^{+} \mathrm{T}$ cells suggested that their accumulation in the adipose was more complicated than what we observed with the $\mathrm{CD} 4^{+} \mathrm{T}$ cells. As such we were wary of reading too much into what their presence in adipose could imply. 
(a) Adipose SVF
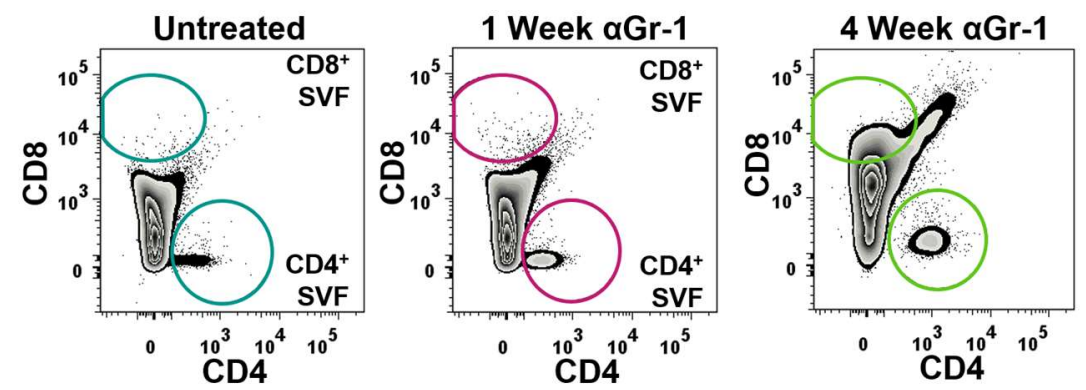

(b)
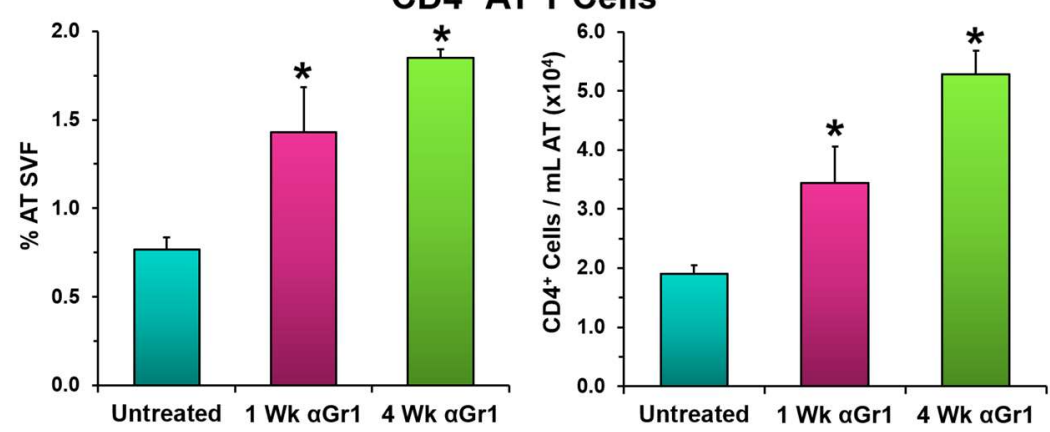

(c)
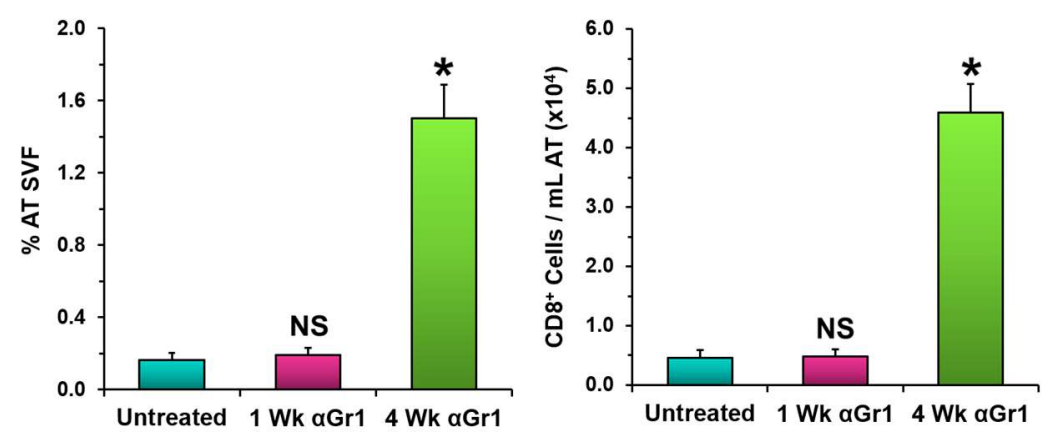

Figure 5.9: MDSC depletion in healthy adipose tissue results in increased numbers of T cells. Healthy, $16-20$-week old C57BI/ 6 mice were treated with $\alpha \mathrm{Gr} 1(15 \mu \mathrm{g} / \mathrm{g})$ every $3-4$ days for 1 or 4 weeks. Epididymal fat pads and spleens were collected one day after the final antibody injection. (a) Zebra plots of depicting $T$ cell populations in AT SVF of untreated and $\alpha \mathrm{Gr} 1$-treated mice. Selected plots for each treatment group were derived from one animal and are representative of their respective group's mean. $(\mathbf{b}-\mathbf{c})$ Frequency (Left) and absolute number (Right) of $\mathrm{CD}^{+}$(b) or $\mathrm{CD}^{+}$(c) T cells in the SVF of aGr1-treated and untreated mice. Data pooled from 4 independent experiments. Bars depict the mean of 11 untreated (Blue), 8 1-week aGr1-treated (Pink), or 6 4-week aGr1-treated mice (Green). Error bars represent SEM. Differences between $\alpha \mathrm{Gr} 1$-treated and untreated animals determined using a Student's $t$-test. *: $P<0.05$ and NS: $P>$ 0.05 . 
(a)

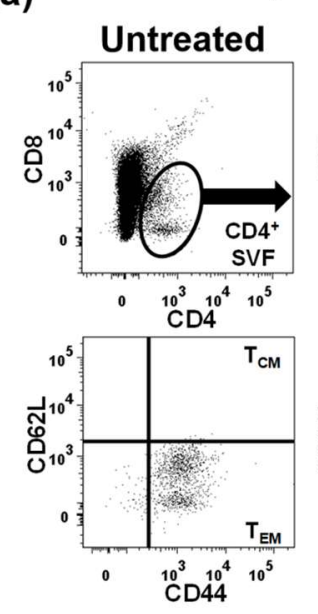

(c)
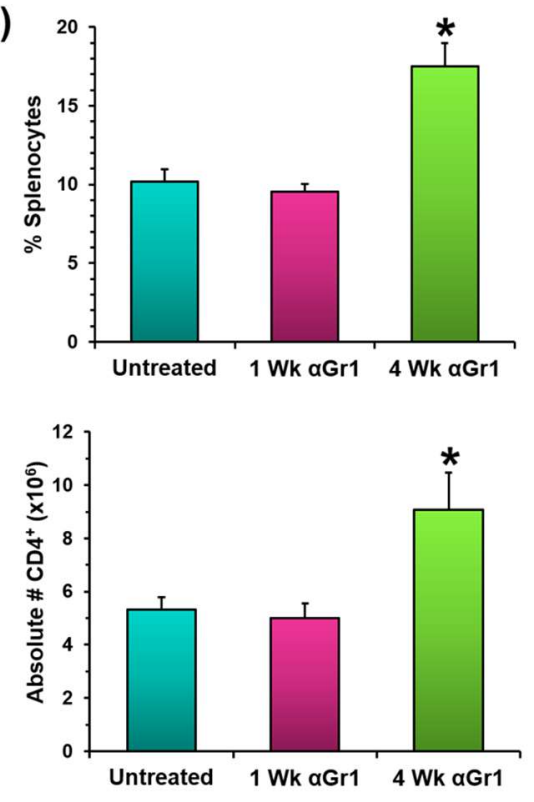

(b)
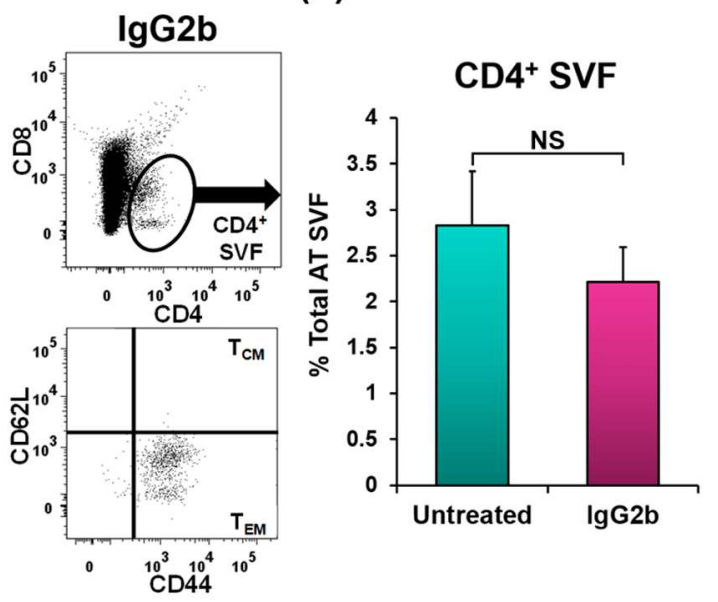

(d)
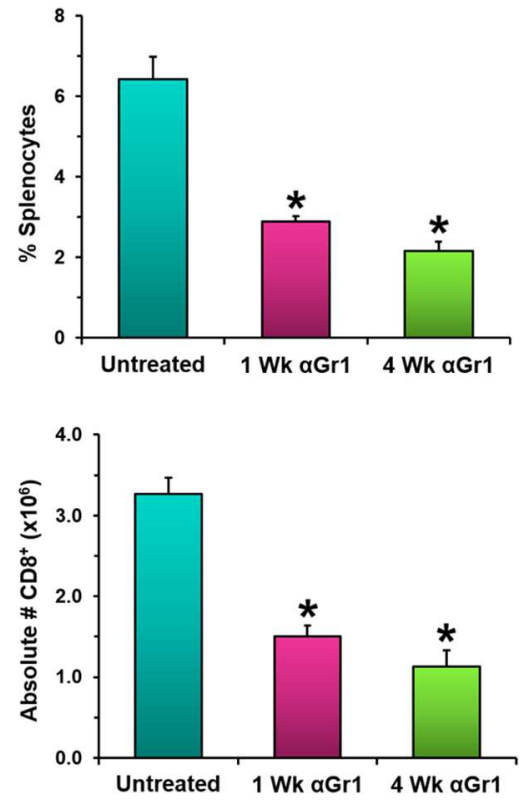

Figure 5.10: $\alpha \mathrm{Gr} 1$ treatment has differential effects on splenic $\mathrm{T}$ cell populations. Healthy, $16-20$-week old C57BI/6 mice were treated with $15 \mu \mathrm{g} / \mathrm{g}$ $\lg 2 \mathrm{~b}(\mathbf{a}-\mathbf{b})$ or $\alpha \mathrm{Gr} 1(\mathbf{c}-\mathbf{d})$ every $3-4$ days for 1 or 4 weeks. Epididymal fat pads and spleens were collected one day after the final antibody injection. (a) Dot plots of T cell characterization in AT of untreated (Left) and IgG2b-treated mice (Right). Selected plots for each treatment group were derived from one animal and are representative of their respective group's mean. (b) Frequency of $\mathrm{CD} 4^{+} \mathrm{T}$ cells in adipose SVF. Bars depict the mean of 4 untreated (Blue) or 5 IgG2b-treated (Pink) from three independent experiments. (c - d) Frequency (Upper) and absolute number (Lower) of splenic $\mathrm{CD}^{+}$(c) or $\mathrm{CD}^{+}$(d) T cells in aGr1-treated and untreated mice. Data pooled from 4 independent experiments. Bars depict the mean of 11 untreated (Blue), 8 1-week aGr1-treated (Pink), or 6 4-week aGr1treated mice (Green). Error bars represent SEM. Differences between treated and untreated animals determined using a Student's $t$-test. *: $P<0.05$ and NS: $P>$ 0.05 . 


\section{Accumulated $T$ cells are effector memory $T$ cells.}

T cell accumulation is often an early indicator of the chronic inflammation seen in obesity. Specifically, activated $\mathrm{CD} 4^{+} \mathrm{T}$ cells are one of the first cell types to infiltrate the tissue during the early stages of this disease. With this in mind, we chose to focus our subsequent studies on comparing our accumulated $\mathrm{CD} 4^{+} \mathrm{T}$ cells to those found in obese animals. Using flow cytometry, we investigated the phenotype of these cells following acute and chronic MDSC depletion. In agreement with what others have described, we observed a small population of $\mathrm{CD}^{+} \mathrm{T}$ cells in the AT of our healthy mice $[39,50]$. In contrast however, only a relatively small percentage of the $\mathrm{CD} 4^{+}$cells were Foxp $3^{+}$regulatory $\mathrm{T}$ cells ( $\mathrm{T}_{\text {Regs }}$ ) $[49,50]$ (Figure 5.11 a). This disparity is most likely due to the kinetics of $T_{\text {Reg }}$ accumulation in AT, as their population begins to expand around $15-20$ weeks of age $[49,50]$. We chose to use $16-20$-week-old mice in the present study as this age range is the human equivalent of middle adulthood when obesity often begins to develop, while others typically use mice which are $25-30$ weeks old. Thus, the fewer than expected $T_{\text {Reg }}$ numbers likely reflects this younger age, as $T_{\text {Reg }}$ populations have not yet begun to expand in the adipose tissue of these healthy mice.

In untreated mice, $86.4 \pm 2.41 \%$ of the $\mathrm{CD} 4^{+} \mathrm{T}$ cell compartment in AT was composed of Foxp3- CD44 ${ }^{+}$CD62 Low $\mathrm{T}$ effector memory cells (TEM). The predominance of the $\mathrm{T}_{\mathrm{EM}}$ phenotype among $\mathrm{CD}^{+}$adipose tissue $\mathrm{T}$ cells was unaffected by MDSC depletion at any time point (1-week $\alpha \mathrm{Gr} 1: 83.9 \pm 1.9 \%$ of $\mathrm{CD}^{+} \mathrm{T}$ cells; 4 -week $\alpha \mathrm{Gr} 1: 84.8 \pm 1.3 \%$ of $\mathrm{CD}^{+}{ }^{+} \mathrm{T}$ cells) (Figure 5.11 b). However, acute and chronic MDSC depletion markedly increased the absolute number of 
$\mathrm{CD}^{+}{ }^{+} \mathrm{T}_{\mathrm{EM}}$ in AT from $1.63 \pm 0.1 \times 10^{4} \mathrm{TEM} / \mathrm{mL}$ AT to $2.91 \pm 0.6 \times 10^{4} \mathrm{TEM} / \mathrm{mL}$ AT and $4.47 \pm 0.3 \times 10^{4} \mathrm{~T}_{\mathrm{EM}} / \mathrm{mL}$ AT, respectively, (Figure 5.11 c), indicating that the accumulation of $\mathrm{CD} 4^{+} \mathrm{T}$ cells in the AT of $\alpha \mathrm{Gr} 1$-treated mice was limited to this effector memory subset.

$56.9 \pm 4.0 \%$ of splenic CD4 ${ }^{+} \mathrm{T}$ cells $\left(2.99 \pm 0.3 \times 10^{6} \mathrm{~T}_{\mathrm{EM}} / \mathrm{spleen}\right)$ exhibited a CD44+ ${ }^{+}$CD2L- $\mathrm{TEM}_{\mathrm{EM}}$ phenotype. As in the adipose, there was no change in the proportion of $\mathrm{T}_{\mathrm{EM}}$ within the total $\mathrm{CD} 4^{+} \mathrm{T}$ cell population (1-week $\alpha \mathrm{Gr} 1: 61.5 \pm 8.0 \%$ of splenic $\mathrm{CD}^{+}$cells; 4 -week $\alpha \mathrm{Gr} 1: 67.4 \pm 2.0 \%$ of splenic $\mathrm{CD} 4^{+} \mathrm{T}$ cells) in the spleens of aGr1-treated mice compared to controls (Figure 5.12 a - b). This suggests that the increased number of $\mathrm{CD}^{+} \mathrm{T}_{\mathrm{EMS}}$ in the AT of aGr1-treated animals results from the systemic depletion of MDSCs. 
(a)

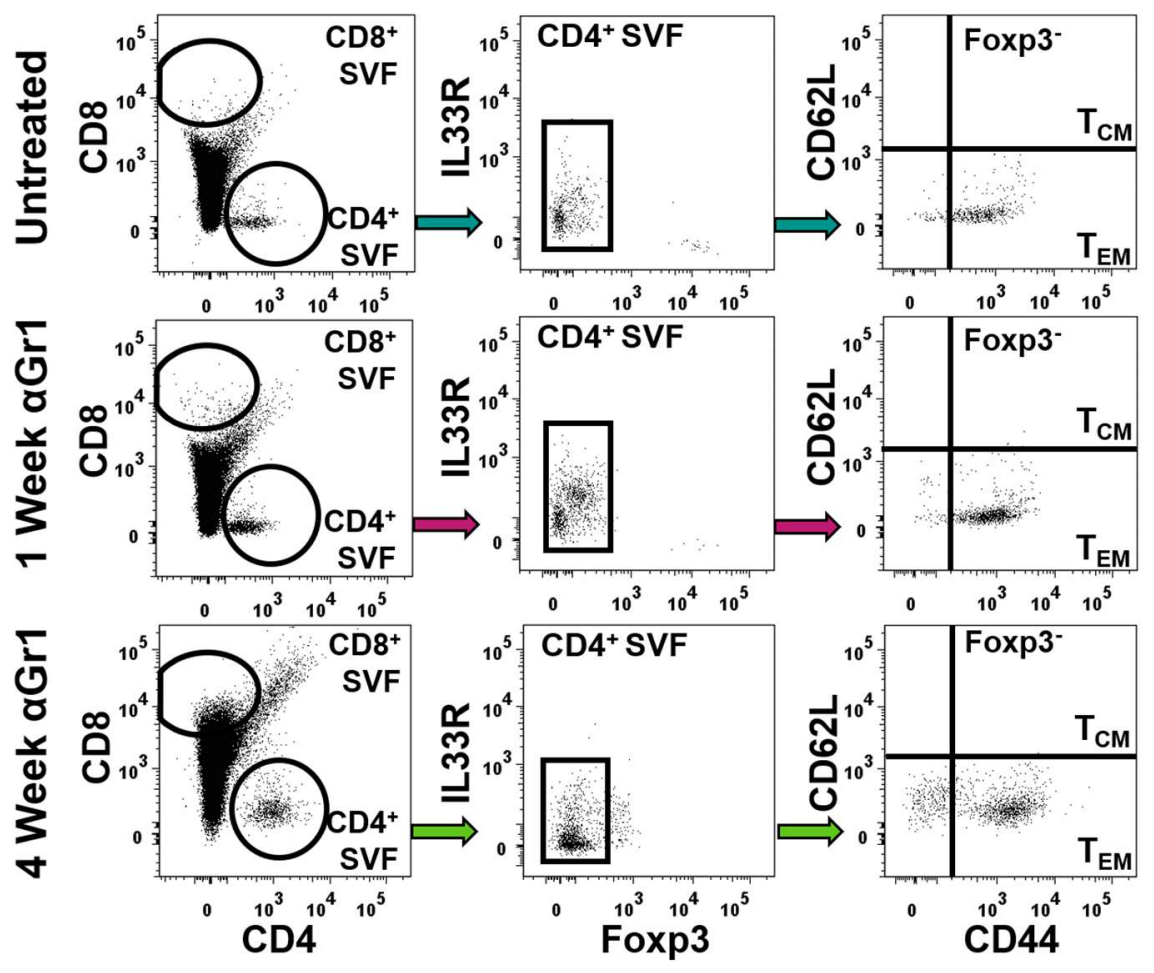

(b)

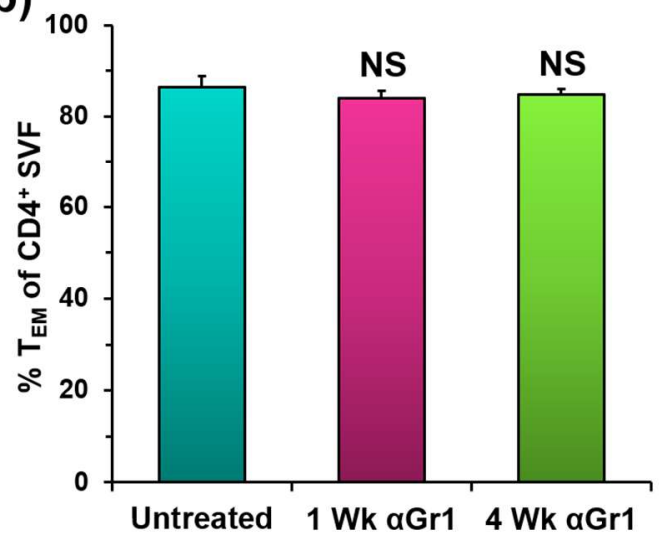

(c) 6.0

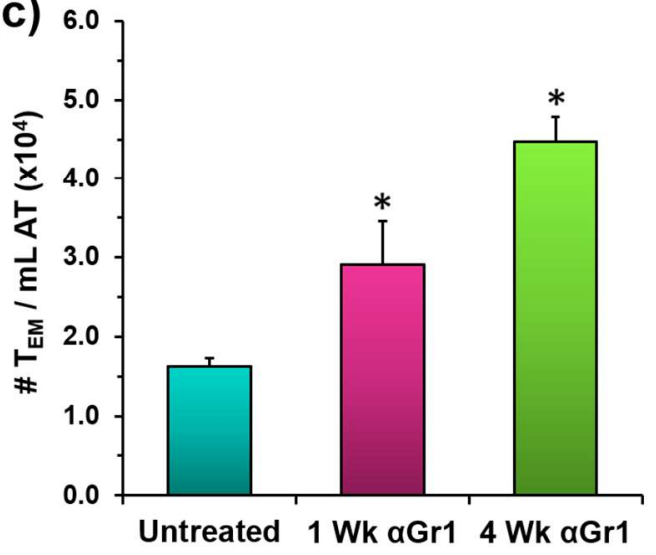

Figure 5.11: Accumulated $T$ cells in adipose following aGr1 treatment are effector memory $\mathrm{T}$ cells. (a) Dot plots depicting the characterization strategy used for $\mathrm{CD}^{+} \mathrm{T}$ cells in AT SVF. Selected plots in each treatment group were derived from one animal and are representative of that group's mean. Тсм: Central Memory T cells. TEM: Effector Memory T cells. (b) Frequency of CD4 ${ }^{+}$SVF cells that were $\mathrm{CD}_{4} 4^{+} \mathrm{CD} 62 \mathrm{~L}^{-} \mathrm{T}_{\mathrm{EM}}$. (c) Absolute number of CD4+ ${ }^{+}$EM in AT SVF. (b c) Data pooled from 4 independent experiments. Bars depict the mean of 11 untreated (Blue), 8 1-week aGr1-treated (Pink), or 6 4-week aGr1-treated mice (Green). Error bars represent SEM. Differences between $\alpha \mathrm{Gr} 1$-treated and untreated animals determined using a Student's $t$-test. *: $P<0.05$ and NS: $P>$ 0.05 . 
(a)

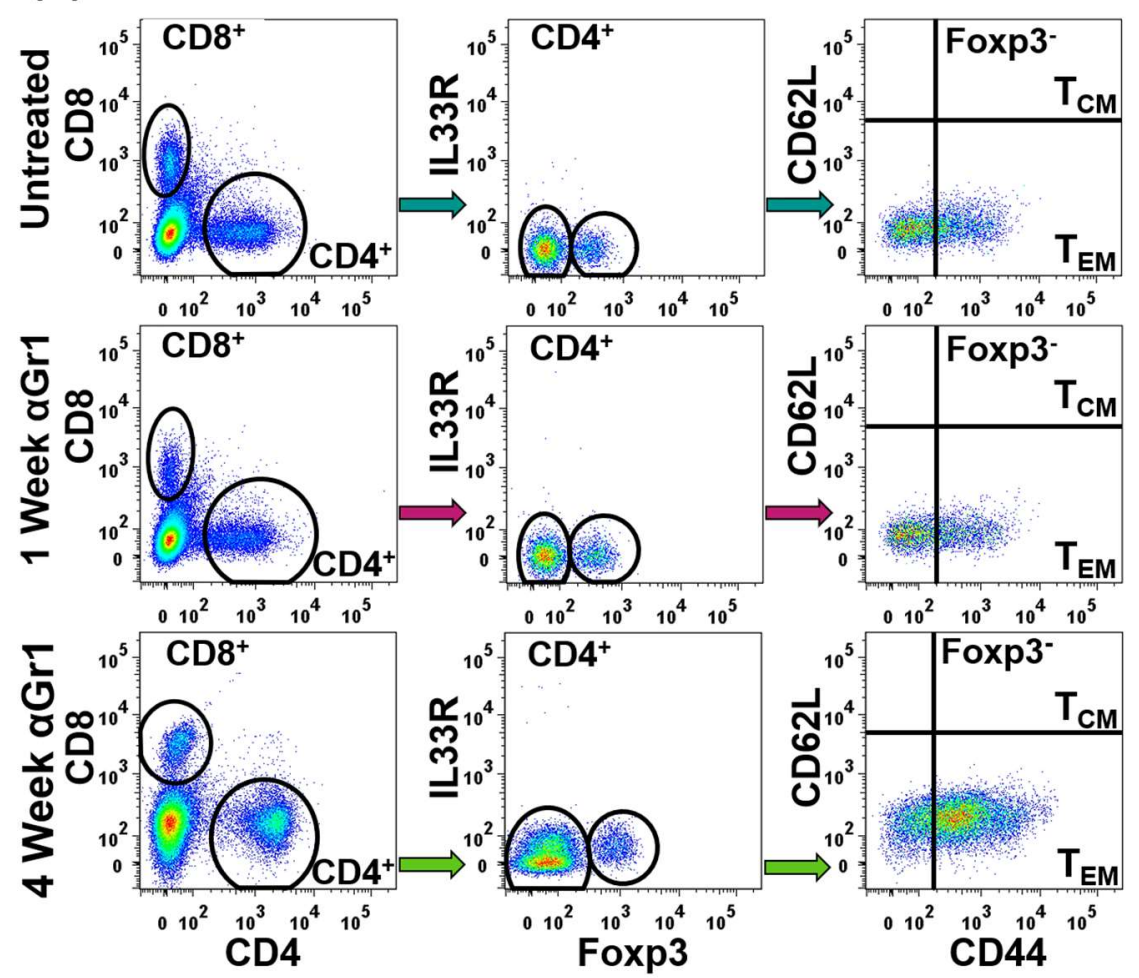

(b)

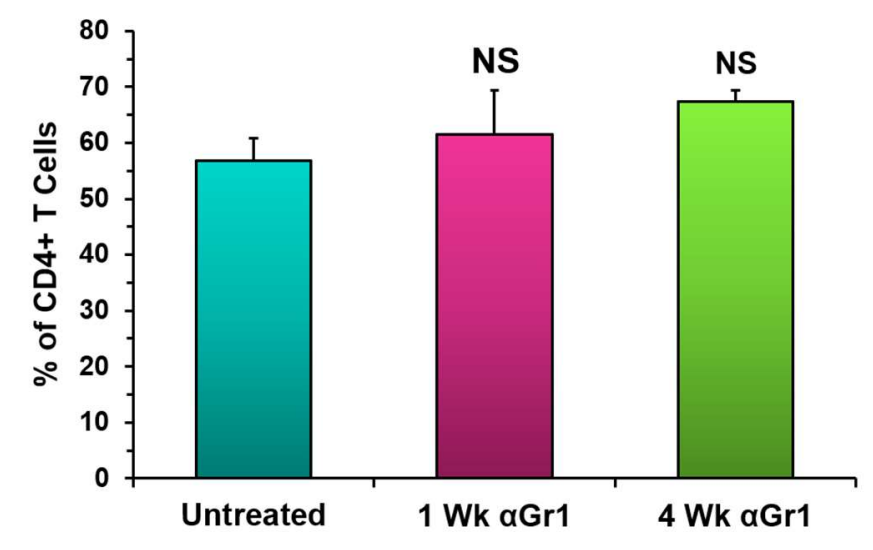

Figure 5.12: The splenic CD4 ${ }^{+} \mathrm{CD} 44^{+} \mathrm{CD} 62 \mathrm{~L}-\mathrm{T}_{\text {Ем }}$ population is unaffected by systemic MDSC depletion. (a) Dot plots depicting the $\mathrm{CD}^{+} \mathrm{T}$ cell characterization strategy in the spleens of untreated (Upper), 1-week aGr1-treated (Middle), and 4-week aGr1-treated mice (Lower). Selected plots in each treatment group were derived from one animal and represent the mean within that group. (b) The frequency of $\mathrm{CD}^{+}$splenocytes that were $\mathrm{CD}_{4} 4^{+} \mathrm{CD} 62 \mathrm{~L}^{-} \mathrm{TEM}$. Data pooled from 4 independent experiments. Bars depict the mean of 11 untreated (Blue), 8 1-week aGr1-treated (Pink), or 6 4-week aGr1-treated mice (Green). Error bars represent SEM. Differences between aGr1-treated and untreated animals determined using a Student's $t$-test. NS: $P>0.05$. 


\section{TEMS upregulate expression of markers associated with activation \& proliferation.}

Most of our knowledge concerning MDSCs comes from studying their function within the tumor microenvironment. In this setting aGr1-mediated MDSC depletion not only increases the number of T cells infiltrating the tumor [177], it also increases their expression of CD69 compared to T cells from non-depleted controls [178]. Additionally, CD69 is one of the first markers to be upregulated on the surface of T cells after they've been activated and has also been associated with $T$ cell retention in a peripheral tissue [179]. Relative to their untreated counterparts, AT-resident $\mathrm{CD} 4^{+} \mathrm{T}_{\mathrm{EM}}$ rapidly upregulated surface expression of this marker after only 1 week of the $\alpha \mathrm{Gr} 1$ treatment (1.65 \pm 0.12 vs $1.0 \pm 0.04)$. CD69 levels were slightly lower in our chronically treated mice $(1.4 \pm 0.12)$ but remained significantly elevated compared to adipose tissue $\mathrm{T}_{\mathrm{EM}}$ isolated from untreated mice (Figure $5.13 \mathrm{a}-\mathbf{b}$ ). CD69 expression was unaffected by treatment with the IgG2b isotype alone (Figure $5.13 \mathrm{c}-\mathrm{d}$ ).

Next, we analyzed Ki-67 expression in the AT SVF cells of 1-week aGr1treated and untreated mice. Compared to controls (Untreated: $18.05 \pm 1.1 \%$; IgG2b: $19.02 \pm 1.2 \%$ ), the percentage of $\mathrm{Ki}^{+} 7^{+} \mathrm{CD} 4^{+}$cells in the adipose significantly increased $(25.8 \pm 0.95 \%)$ following MDSC depletion (Figure 5.14 a b). This strongly suggests that these cells are proliferating in situ rather than being recruited into the tissue from circulation.

To further elucidate the activation state of these $\mathrm{CD} 4^{+}$cells, we evaluated their transcription levels of classic $T$ cell effector cytokines using qRT-PCR. CD11 $\mathrm{b}^{-} \mathrm{CD} 4^{+}$cells, sorted from the SVF of $\alpha \mathrm{Gr} 1$-treated and untreated mice were 
actively transcribing Tnfa at relatively similar levels (Figure $5.15 \mathrm{a}-\mathbf{b}$ ), while cells from aGr1-treated animals showed a trend towards the upregulation of both Ifng and II2 transcription compared to untreated controls. This could indicate that the $T_{E M}$, which accumulate in AT after $\alpha \mathrm{Gr} 1$ treatment, may have been activated by exposure to some constitutively present stimuli within the tissue. However, further experiments using single cell RNAseq or intracellular cytokine staining are necessary to demonstrate true changes in the functionality of these T cells. Taken together it becomes apparent that the adipose-resident MDSCs play a significant part in modulating the expansion of the CD4 ${ }^{+} \mathrm{T}_{\mathrm{EM}}$ population in healthy $\mathrm{AT}$. 
(a)

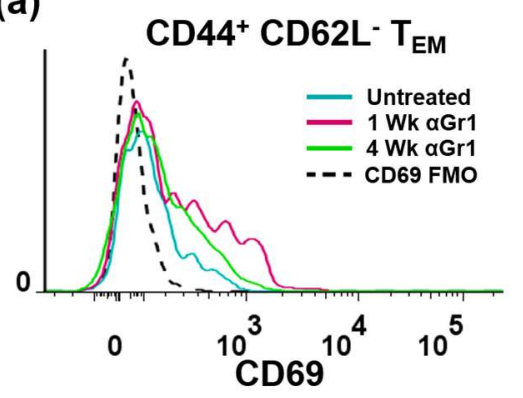

(c)

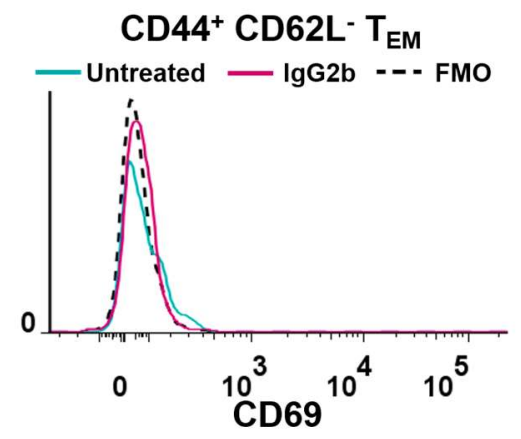

(b)

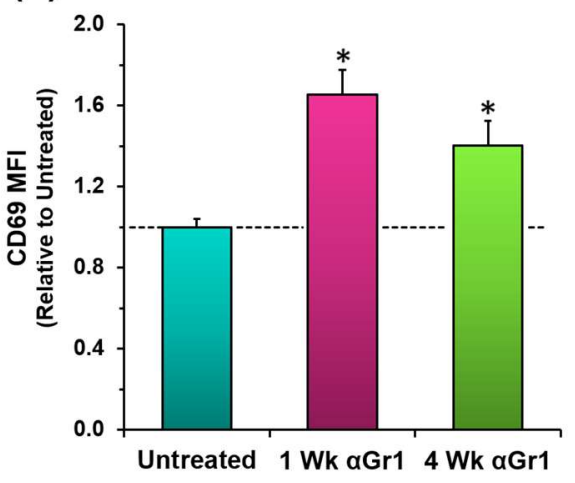

(d)

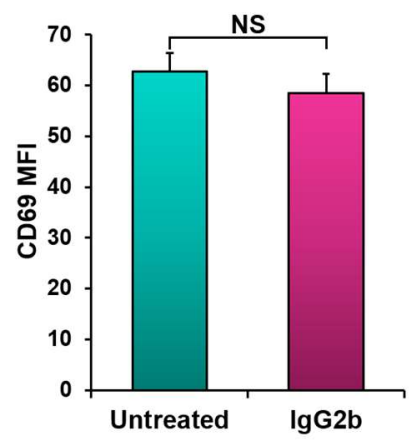

Figure 5.13: $\alpha \mathrm{Gr} 1$ treatment, but not IgG2b treatment, increases CD69 expression on CD4 ${ }^{+} \mathrm{T}_{\mathrm{EM}}$ in adipose SVF. (a) Representative histogram depicting CD69 expression on CD44 ${ }^{+} \mathrm{CD}^{-} \mathrm{L}^{-} \mathrm{TEM}_{\mathrm{E}}$ in the adipose of untreated (Blue line), 1week aGr1-treated (Pink Line), and 4-week aGr1-treated mice (Green Line). Selected histograms for each treatment group were derived from one animal in each group and are representative of that group's mean. The dashed line depicts the fluorescence-minus one control (FMO) for CD69. (b) The geometric mean fluorescence intensity (MFI) of CD69 expression on CD44 ${ }^{+}$CD62L- TEM in the SVF of either 1-week (Pink) or 4-week (Green) aGr1-treated mice relative to untreated controls (Blue). Data pooled from 4 independent experiments. Bars depict the mean of 11 untreated, 8 1-week aGr1-treated, or 6 4-week aGr1-treated mice. Error bars represent SEM. Differences between aGr1-treated and untreated animals determined using a Student's t-test. *: $P<0.05$. (c) Representative histogram of CD69 expression on CD44 ${ }^{+}$CD62L- $\mathrm{TEM}_{\mathrm{EM}}$ in the SVF of either untreated (Blue Line) or lgG2b-treated (Pink Line). Selected histograms for each group were derived from one animal and are representative of that group's mean. The dashed line represents the fluorescence minus one (FMO) control for CD69. (d) The geometric mean fluorescence intensity (MFI) of CD69 expression on CD44 ${ }^{+}$ $\mathrm{CD} \mathrm{L}^{-} \mathrm{T}_{\mathrm{B}}$ in the SVF of either IgG2b-treated (Pink) or untreated (Blue) mice. Data pooled from three independent experiments. Bars depict the mean of 4 untreated or $5 \mathrm{lgG} 2 \mathrm{~b}$-treated mice. Error bars represent SEM. Differences between aGr1-treated and untreated animals determined using a Student's $t$-test. NS: $P$ > 0.05 . 

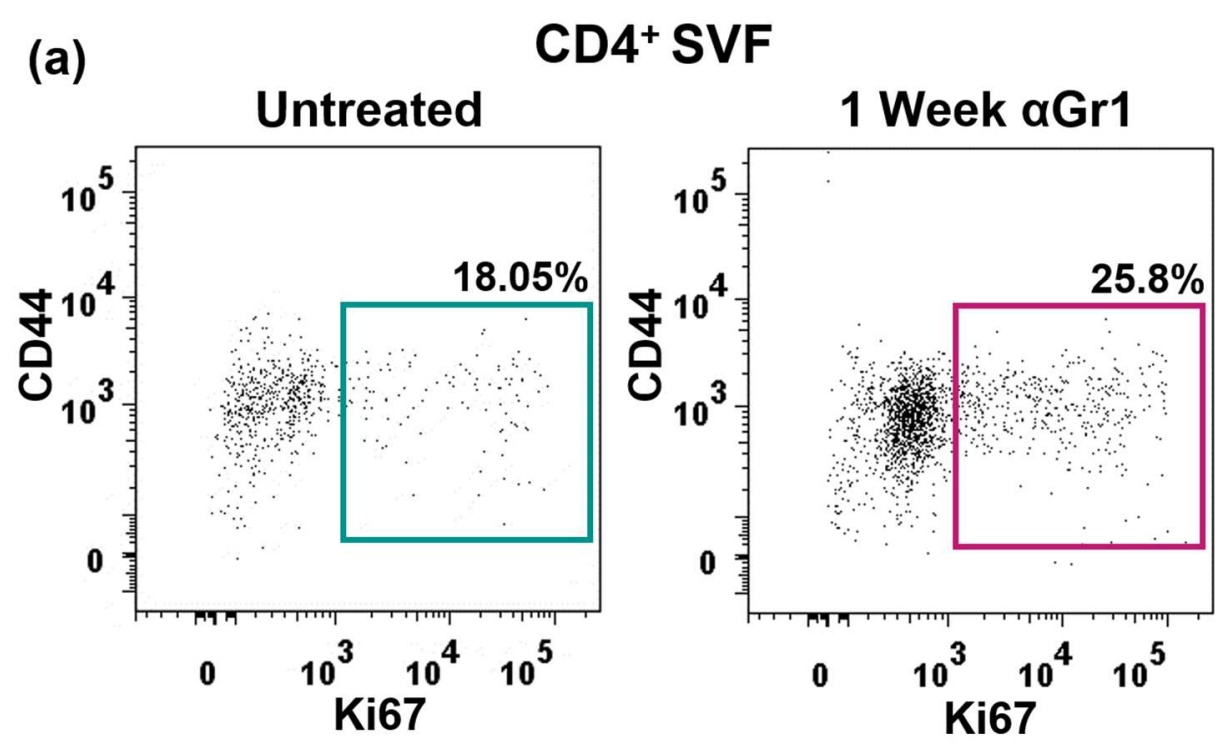

(b)

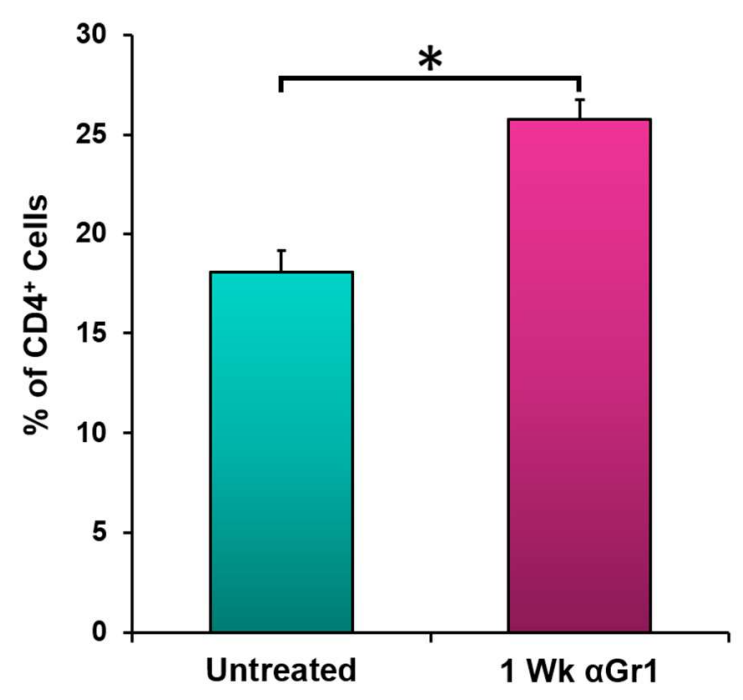

Figure 5.14: $\mathrm{CD}^{+} \mathrm{T}$ cells in the adipose increase their expression of Ki-67 following acute MDSC depletion. Healthy, $16-20$-week old C57BI/6 mice were treated with $\alpha \mathrm{Gr} 1(15 \mu \mathrm{g} / \mathrm{g})$ every $3-4$ days for 1 week. Epididymal fat pads were collected one day after the final antibody injection. $(\mathbf{a}-\mathbf{b})$ Isolated SVF cells were stained for Ki67 expression and analyzed using flow cytometry. (a) Representative dot plots of $\mathrm{CD}^{+} \mathrm{T}$ cells in adipose tissue (AT) stromal vascular fraction cells (SVF) of untreated (Left) and aGr1-treated mice (Right). Gate depicts Ki-67 expression by $\mathrm{CD}^{+} \mathrm{SVF}$ cells within each treatment group. Selected plots were derived from one animal and are representative of their respective group's mean. (b) Frequency of $\mathrm{CD} 4^{+}$SVF cells that were $\mathrm{Ki}-67^{+}$. Bars depict the mean of 4 untreated and 4 $\alpha \mathrm{Gr} 1$-treated mice from 3 independent experiments. Differences between aGr1treated and untreated animals determined using a Student's $t$-test. *: $P<0.05$. 
(a)

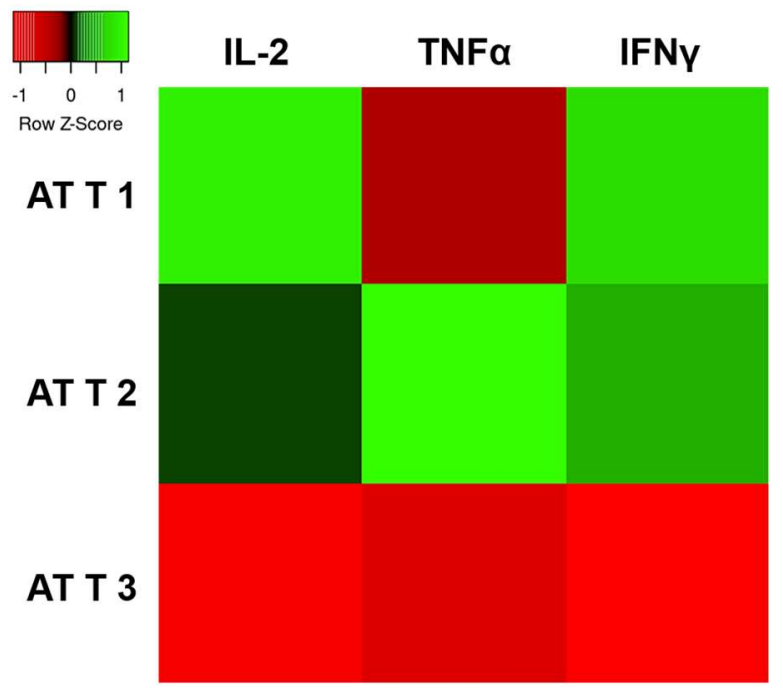

(b)

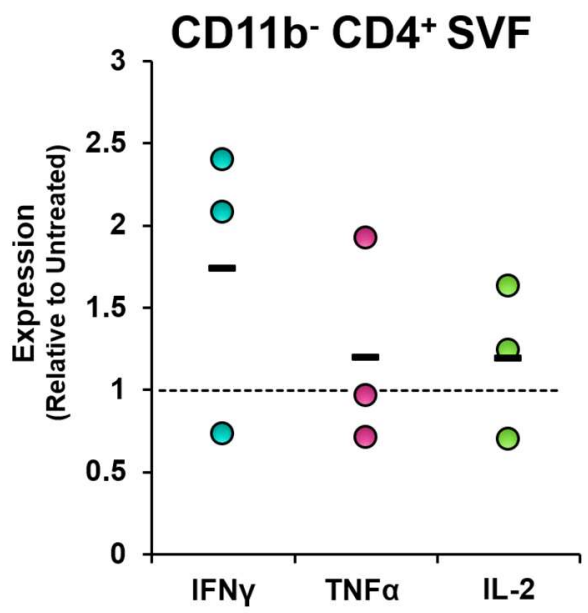

Figure 5.15: $\mathrm{CD4}^{+} \mathrm{T}$ cells may upregulate IFNy following MDSC depletion. mRNA was collected from CD11b- CD4 ${ }^{+}$SVF cells isolated from 1-week aGr1treated mice. (a) Heatmap of genes expressed by CD11b- CD4 ${ }^{+}$SVF T cells isolated from $\alpha \mathrm{Gr} 1$-treated mice. Differential expression determined relative to untreated controls. (b) qRT-PCR was used to determine their expression of Ifng (IFNY), Tnfa (TNFa), or II2 (IL-2) relative to $\mathrm{CD}^{+}{ }^{+} \mathrm{T}$ cells in the AT of untreated mice (Dashed Line). $\Delta C_{\text {T }}$ calculated relative to Tbp transcription. Each circle represents an independent experiment where cells were sorted from the pooled SVF of five untreated or five $\alpha \mathrm{Gr} 1$-treated mice. Differences between treated and untreated animals determined using a student's $t$-test. *: $P<0.05$. 


\section{Adipose-resident macrophages develop a "mixed" phenotype following MDSC depletion.}

In addition to inhibiting $T$ cell proliferation, MDSCs can also modulate the function and phenotype of other cells in order to suppress inflammatory responses [107]. In the tumor microenvironment, crosstalk with MDSCs promotes the protumor, immunosuppressive nature of tumor-associated macrophages [114]. Since AT-resident macrophages (ATMs) are an integral component of the AT immune cell network [20], we investigated whether, similar to the tumor microenvironment, adipose-resident MDSCs promoted the "M2"-like, suppressive phenotype of ATMs.

As before, we analyzed the adipose stromal cells from aGr1-treated or untreated mice using flow cytometry to detect any overall changes in the make-up of the AT myeloid cell compartment. CD11 $\mathrm{b}^{+} \mathrm{CD} 301^{+} \mathrm{MHC}-\mathrm{II}^{+} \mathrm{ATMs}$ represented $13.16 \pm 0.9 \%$ of the total SVF $\left(3.62 \pm 0.4 \times 10^{5}\right.$ cells $/ \mathrm{mL}$ AT $)$ in healthy untreated mice. Both the proportion (1-week $\alpha \mathrm{Gr} 1: 13.81 \pm 0.9 \%$ of total SVF; 4-week $\alpha \mathrm{Gr} 1$ : $10.67 \pm 0.6 \%$ of total SVF) and absolute number (1-week $\alpha \mathrm{Gr} 1: 3.42 \pm 0.4 \times 10^{5}$ ATMs $/ \mathrm{mL}$ AT; 4-week $\alpha \mathrm{Gr} 1: 3.35 \pm 0.3 \times 10^{5} \mathrm{ATMs} / \mathrm{mL}$ AT) of these cells were unaffected by the $\alpha \mathrm{Gr} 1$ treatment (Figure $5.16 \mathbf{a}-\mathbf{b}$ ). Similarly, we did not observe difference in frequency or number of ATMs in the SVF of IgG2b treated controls (Figure $5.16 \mathrm{c}$ ).

Acute MDSC depletion did, however, lead to a marked upregulation of MHC-II and CD11c on these cells, as indicated by a significant increase in the relative geometric MFI of these two surface markers compared to ATMs from their untreated counterparts (MHC-II: $1.0 \pm 0.07$ vs $2.11 \pm 0.31$ and CD11c: $1.0 \pm 0.02$ 
vs $1.35 \pm 0.02$ ) (Figure $5.17 \mathbf{a}-\mathbf{b}$ ). Additionally, there was a trending decrease in the MFI of the "M2"-like marker CD301 on the surface of this same population (1.0 \pm 0.17 vs $0.55 \pm 0.23$ ) (Figure $5.17 \mathbf{a}-\mathbf{b}$ ). $\lg \mathrm{g} 2 \mathrm{~b}$ treatment had no impact on the expression of these surface markers relative to untreated controls (MHC-II: $1.0 \pm$ 0.1 vs $1.08 \pm 0.09$ and CD11c: $1.0 \pm 0.04$ vs $0.92 \pm 0.05$ ) (Figure 5.18 a). Notably, similar alterations in the expression of these ATM surface markers are hallmarks of the immune dysregulation that occurs in the early stages of obesity development $[21,29]$.

ATM surface marker expression following sustained, 4-week ablation of MDSCs paints a more nuanced picture of AT immune cell dynamics. We found a three-fold increase in their expression of MHC-II relative to untreated mice (1.0 \pm 0.07 vs $3.24 \pm 0.5)($ Figure $\mathbf{5 . 1 8} \mathbf{b}-\mathbf{c}$ ), while CD11c expression dropped from what we observed in 1-week $\alpha$ Gr1-treated mice and was no longer different from the levels in untreated mice $(1.0 \pm 0.02$ vs $0.91 \pm 0.07)$ (Figure $5.18 \mathbf{b}-\mathbf{c})$. The restoration of $\mathrm{CD} 11 \mathrm{c}$ expression concomitant with increasing MHC-II levels add another layer of nuance to our understanding of immune cell dynamics in adipose tissue. These results perfectly illustrate the plasticity and adaptability of the immune cell network in healthy adipose tissue revealing the dynamic changes ATMs can undergo overtime as work to restore of immune homeostasis in the tissue. Importantly, the ever-increasing levels of MHC-II on the surface of ATMs also demonstrates the integral part MDSCs play in healthy adipose as the remaining AT immune cells seem unable to fully return to their original, pre- $\alpha \mathrm{Gr} 1$ phenotype. Taken together, this suggests that the adaptability of the immune cell 
compartment in healthy adipose tissue relies upon the crosstalk between these cells and the loss of any single member weakens the whole network. 


\section{CD11b+CD301+ATMs}
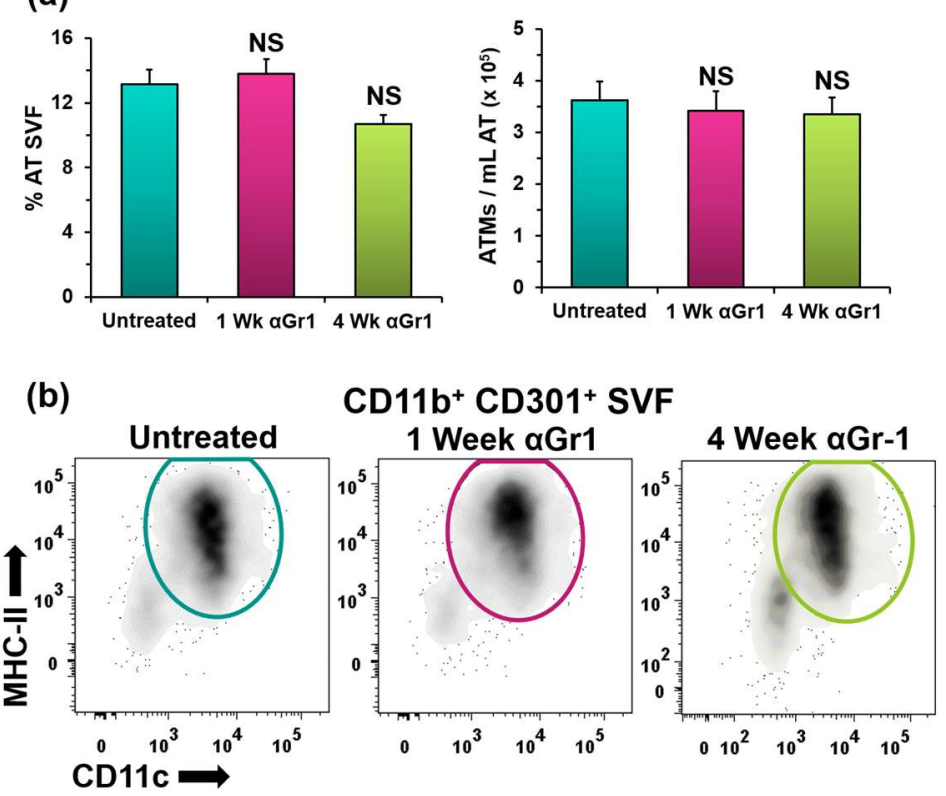

(c)
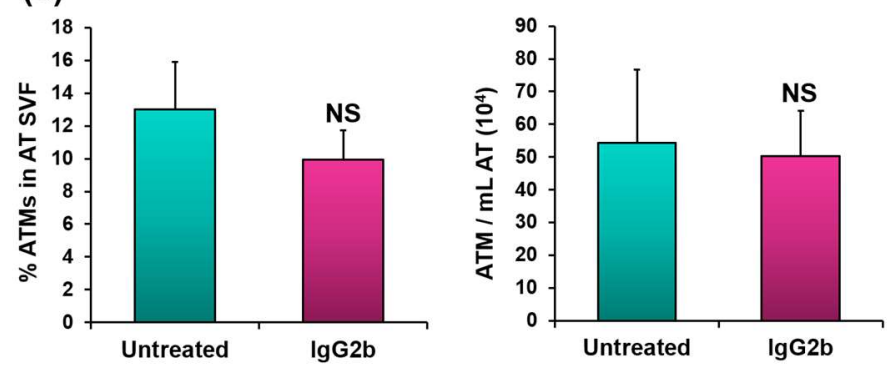

Figure 5.16: $\alpha \mathrm{Gr} 1$ treatment does not affect the number or proportion of adipose tissue-resident macrophages. Healthy, $16-20$-week old C57BI/6 mice were treated with $15 \mu \mathrm{g} / \mathrm{g}$ aGr1 $(\mathbf{a}-\mathbf{b})$ or IgG2b (c) every $3-4$ days for 1 or 4 weeks. Epididymal fat were collected one day after the final antibody injection. (a) Frequency (Left) and absolute number (Right) of CD11 $\mathrm{b}^{+} \mathrm{CD} 301^{+}$adipose tissueresident macrophages (ATMs) in the total AT SVF. Data pooled from 4 independent experiments. Bars depict the mean of 11 untreated (Blue), 8 1-week aGr1-treated (Pink), or 6 4-week aGr1-treated mice (Green). Error bars represent SEM. Differences between $\alpha \mathrm{Gr} 1$-treated and untreated animals determined using a Student's $t$-test. NS: $P>0.05$. (b) Representative density plots of CD11c and $\mathrm{MHC}-\mathrm{Il}$ expression on $\mathrm{CD} 11 \mathrm{~b}^{+} \mathrm{CD} 301^{+}$cells in untreated and $\mathrm{aGr} 1$-treated mice. Selected plots in each treatment group were derived from one animal and represent the mean within that group. (c) Frequency of ATMs in adipose SVF from untreated (Blue) or 5 lgG2b-treated (Pink) mice. Data pooled from three independent experiments with a total of 4 untreated or $5 \mathrm{lgG} 2 \mathrm{~b}$-treated animals. Error bars represent SEM. Differences between treated and untreated animals determined using a Student's $t$-test. NS: $P>0.05$. 

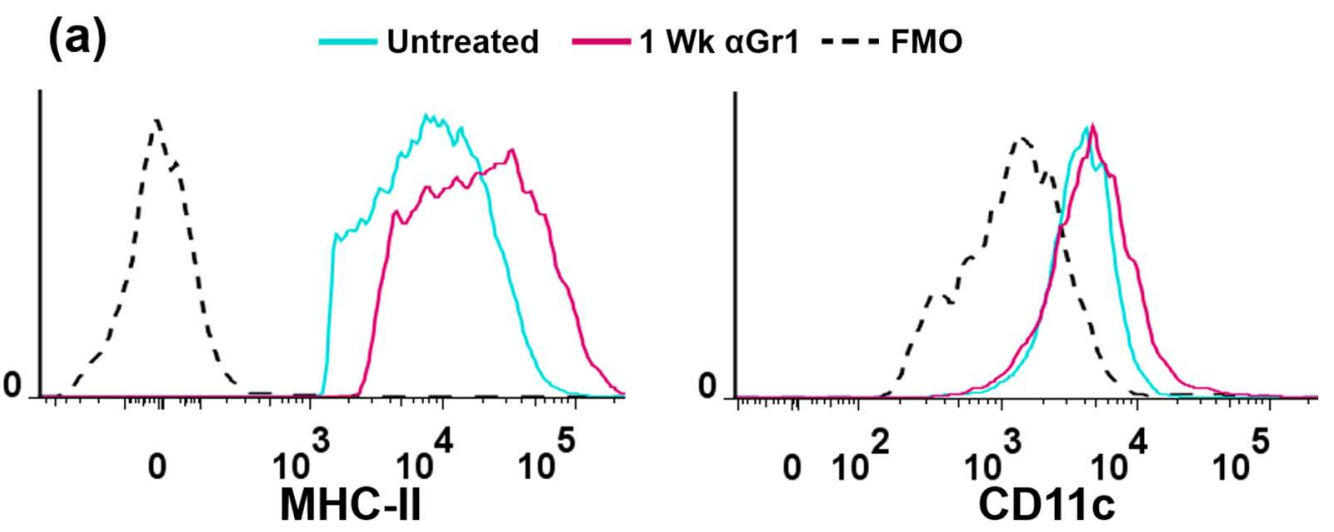

(b)
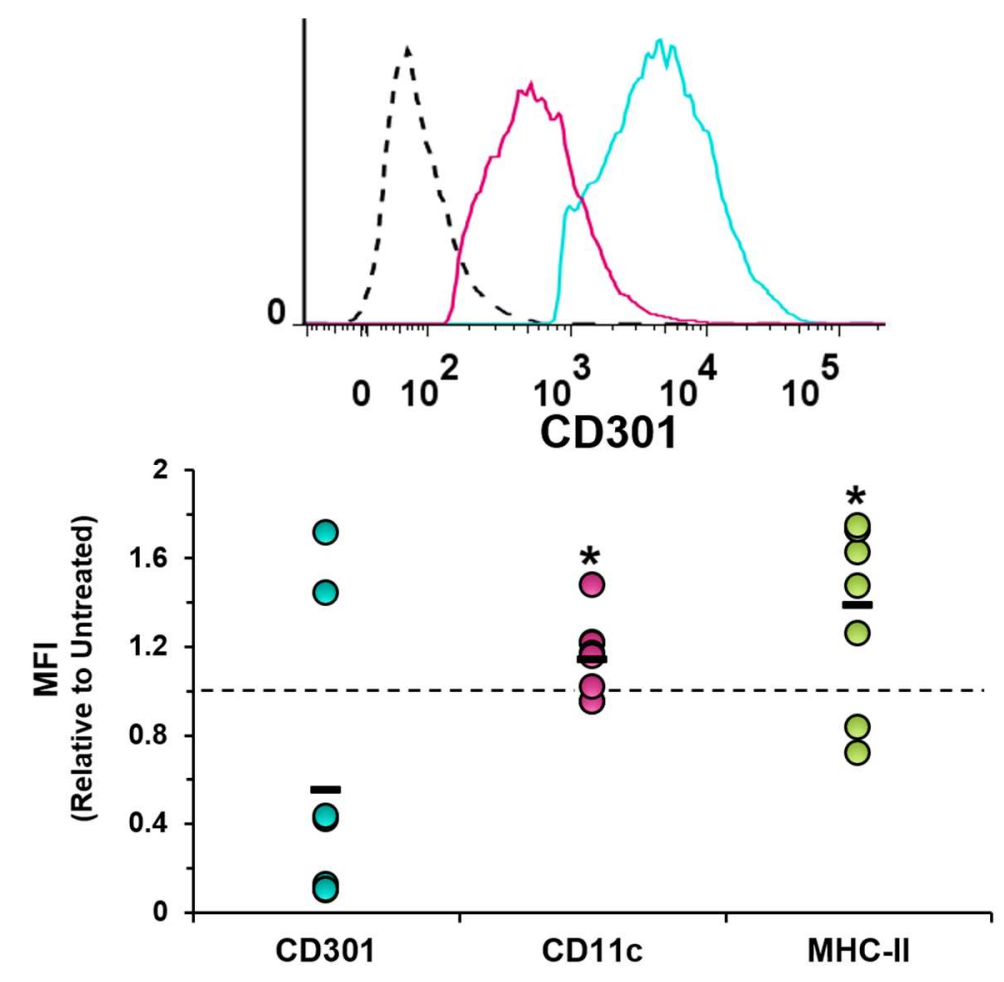

Figure 5.17: ATM surface marker expression is altered by acute MDSC depletion. Healthy, 16 - 20 -week old C57BI/ 6 mice were treated with $15 \mu \mathrm{g} / \mathrm{g}$ aGr1 every 3 - 4 days for 1 week. (a) Representative histograms depicting MHC-II, CD11C, and CD301 surface expression on CD11 $b^{+}$CD $301^{+}$SVF cells in untreated (Blue Line) and 1-week aGr1-treated (Pink Line) mice. The dashed line depicts the fluorescence-minus one control (FMO) for the indicated surface marker. Each plot depicts one mouse from each group and is representative of the mean within that group. (b) Geometric mean fluorescence intensity (MFI) of each surface marker on $\mathrm{CD} 11 \mathrm{~b}^{+} \mathrm{CD} 301^{+}$ATMs in 1-week $\alpha \mathrm{Gr} 1$-treated mice (Circles) relative to untreated mice (Dashed Line). Horizontal bars represent the mean of 8 aGr1-treated animals. Differences between treated and untreated animals determined using a Student's $t$-test. *: $P<0.05$. 


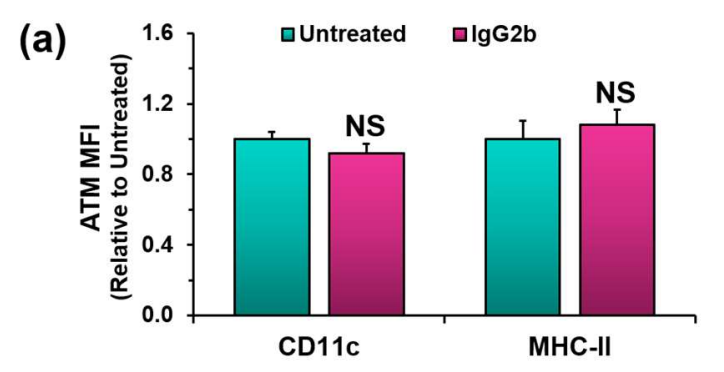

(b) — Untreated - 1 wk aGr1 - 4 Wk aGr1 -- - FMO

(c)
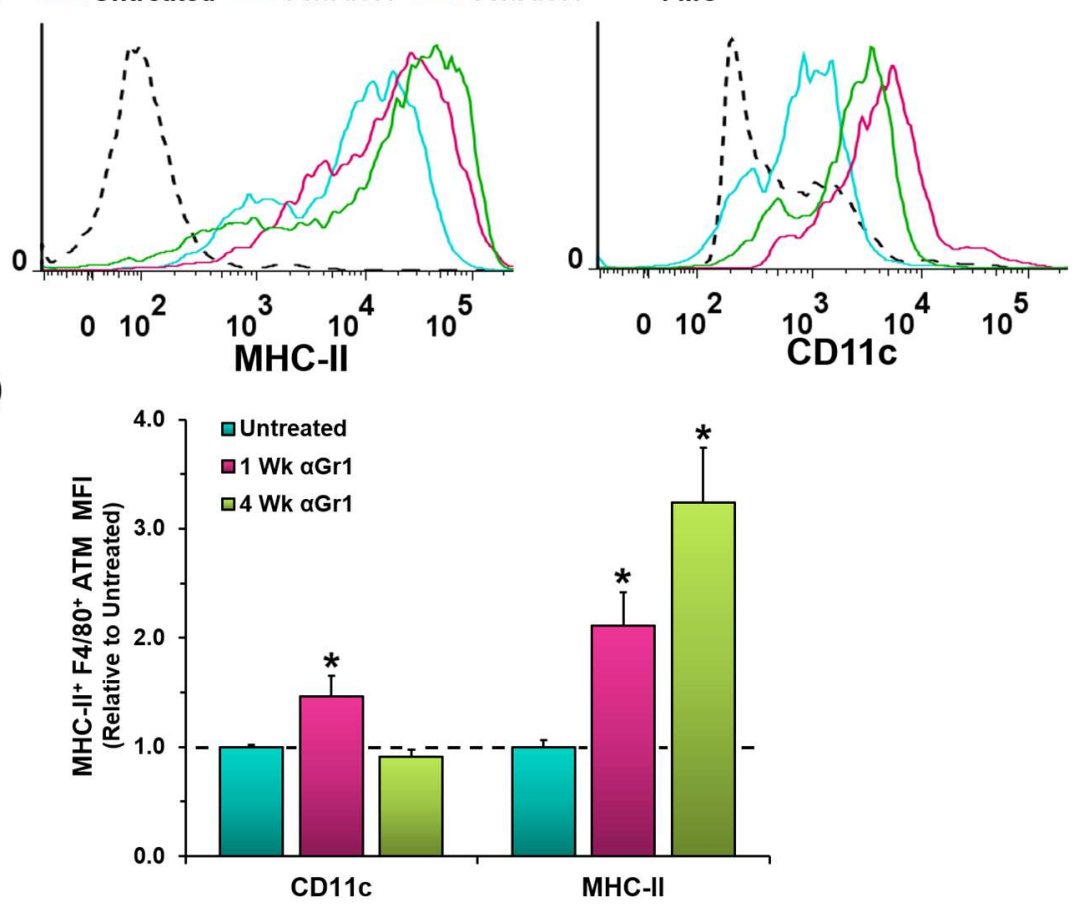

Figure 5.18: ATMs differentially respond to acute and chronic MDSC depletion. Healthy, $16-20$-week old C57Bl/6 mice were treated with $15 \mu \mathrm{g} / \mathrm{g}$ IgG2b (a) or aGr1 (b - c) every 3 - 4 days for 1 or 4 weeks. (a) Geometric mean fluorescence intensity (MFI) of indicated surface markers on CD11 $\mathrm{b}^{+} \mathrm{CD} 301^{+}$ ATMs from untreated (Blue) and IgG2b-treated (Pink) mice. Differences between treated and untreated animals determined using a Student's $t$-test. NS: $P>0.05$. (b) Representative histograms of MHC-II (Left) and CD11c (Right) surface expression by CD11 $\mathrm{b}^{+} \mathrm{F} 4 / 80^{+} \mathrm{MHC}-\mathrm{II}^{+}$ATMs in untreated (Blue Line), 1-week (Pink Line), and 4-week (Green Line) aGr1-treated mice. The dashed line depicts the fluorescence-minus one control (FMO) for the indicated surface marker. Each plot depicts one mouse from each group and is representative of the mean within that group. (c) MFI of CD11c \& MHC-II on CD11 b $\mathrm{b}^{+} \mathrm{F} / 80^{+} \mathrm{MHC}-\mathrm{II}^{+} \mathrm{ATMs}$ following 1 or 4 weeks of $\alpha \mathrm{Gr} 1$ treatment. Data pooled from four independent experiments. Bars depict the mean of 11 untreated (Blue), 81 -week aGr1-treated (Pink), or 6 4week aGr1-treated mice (Green). Error bars represent SEM. Differences between aGr1-treated and untreated animals determined using a Student's $t$-test. *: $P<$ 0.05 . 


\section{ATM gene expression is unaffected by MDSC depletion.}

While intriguing, changes in surface marker expression alone were not enough to conclude that aMDSCs were controlling the immunomodulatory functions of the ATMS. To determine if in vivo MDSC depletion led to functional changes in the AT macrophage population, gene expression analysis using qPCR was performed on $\mathrm{CD} 11 \mathrm{~b}^{+} \mathrm{CD} 301^{+} \mathrm{MHC}-\mathrm{II}^{+}$ATMs sorted from the AT SVF of either aGr1-treated or untreated mice. Our analysis focused on some of the key pro- and anti-inflammatory cytokines associated with ATMs in health and disease including TNFa, IL-10, and TGF $\beta$. Although there was a trend towards the downregulation of $/ / 10$ and upregulation of Tnfa in aGr1-treated ATMs relative to untreated controls, there was no significant difference in the overall transcript levels of these cytokines between the two groups (Figure 5.19). In obesity, ATMs exhibiting surface marker changes similar to those we observed also upregulate Arginase transcription. This was not the case for the ATMs we isolated from MDSC-depleted mice. Finally, we looked at expression of the transcription factor PPARy, which is strongly associated with the immunomodulatory nature of many AT-resident cells including ATMs [180-182]. Again, ATM transcription of this gene was unaffected by the loss of MDSCs (Figure 5.19). Taken together, our findings suggest that the loss of aMDSCs, in vivo, leads to phenotypical but not functional changes in AT resident macrophages; however, further studies using intracellular cytokine staining or single cell RNAseq are necessary to definitively determine this. 
(a)

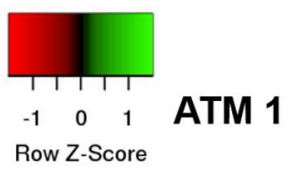

ATM 2

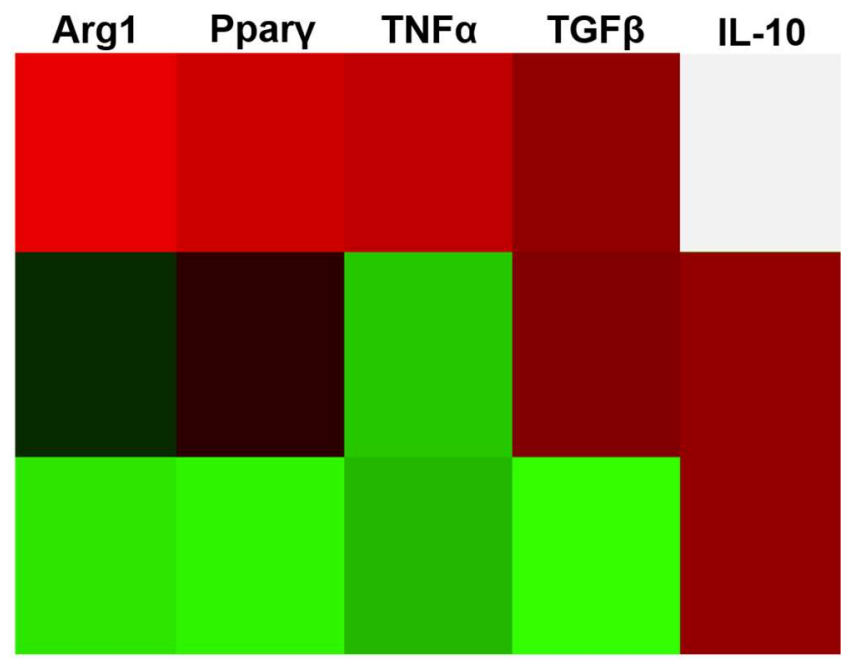

(b)

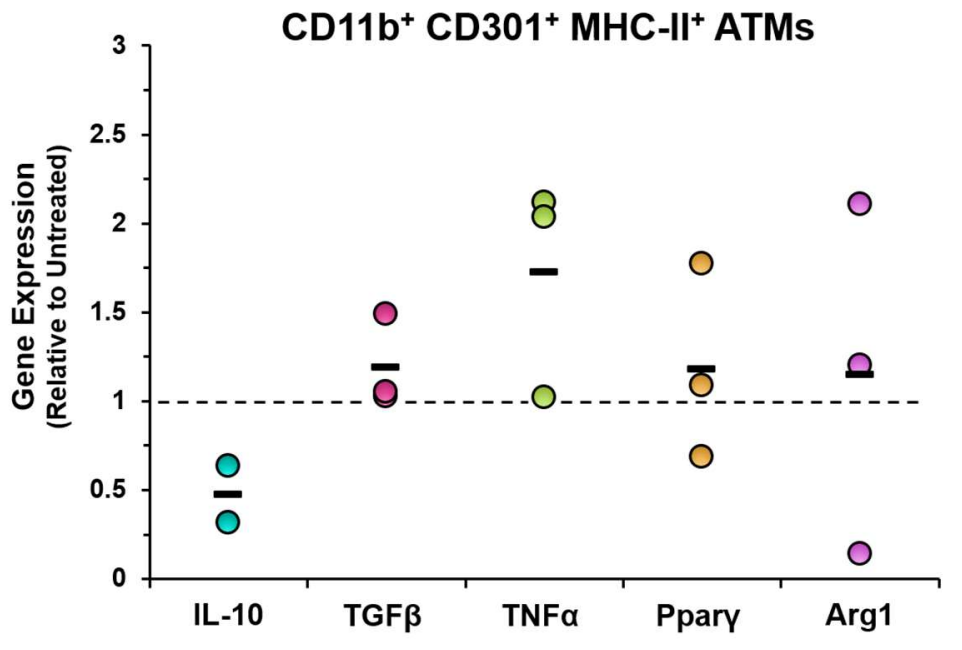

Figure 5.19: ATM expression of immunomodulatory genes remains unchanged following $\alpha \mathrm{Gr} 1$ treatment. mRNA was collected from the CD11 $\mathrm{b}^{+}$ CD $301^{+} \mathrm{MHC}-\mathrm{II}^{+}$ATMs isolated from 1-week aGr1-treated mice. (a) Heatmap of genes expressed by CD $11 \mathrm{~b}^{+} \mathrm{CD} 301^{+} \mathrm{MHC}-\mathrm{II}^{+} \mathrm{ATMs}$ isolated from aGr1-treated mice. Differential expression determined relative to untreated controls. (b) qRTPCR was used to determine their expression of II10 (IL-10), Tgfb1 (TGF $\beta$ ), Tnfa (TNFa), Pparg (PPARY), or Arg1 relative to ATMs in the AT of untreated mice (Dashed Line). $\triangle \mathrm{C}_{\mathrm{T}}$ calculated relative to $T b p$ transcription. Each circle represents an independent experiment where cells were sorted from the pooled SVF of five untreated or five aGr1-treated mice. Differences between treated and untreated animals determined using a student's $t$-test. *: $P<0.05$. 


\section{Adipose-resident MDSCs regulate whole adipose homeostasis \& immune cell dynamics.}

Since ATMs and $\mathrm{CD}^{+}{ }^{+} \mathrm{T}$ cells represent just two players in a large, heterogeneous network of immune cells, we wondered if aMDSCs could be interacting with other AT stromal cells in any meaningful way. For these studies we examined gene transcription in the full SVF isolate to assess how the loss of MDSCs affected the other non-ATM or T cell components of the AT immune cell network. Along a similar vein, used explored transcriptional changes in whole adipose tissue isolated $\alpha \mathrm{Gr} 1$-treated mice to get an idea of what was occurring on the whole tissue level to determine if dysregulating this network even slightly had downstream consequences on the tissue's health.

We focused on the key cytokines, enzymes, and transcription factors whose transcript levels are known to fluctuate with the inflammatory state of the tissue. Surprisingly, we found that, relative to untreated controls, aGr1-treated mice had increased transcript levels of immune suppressive cytokines. I/10 and Tgfb1 were significantly upregulated in the SVF and AT, respectively, following MDSC depletion (Figure 5.20 a - b). Additionally, in the absence of aMDSCs, transcription of $/ / 33$ was also significantly upregulated in both the SVF and whole AT compared to intact controls (Figure $5.20 \mathbf{a}-\mathbf{b}$ ). This cytokine is of particular note since it is heavily involved in regulating the ILC2 - ATM arm of the AT immune cell network [30, 31, 182]. However, in both MDSC-depleted SVF and AT, relative expression of Pparg was no different from that of untreated mice, similar to what we observed with the ATMs. 
In contrast to this apparent increase in the suppressive nature of the AT microenvironment following MDSC depletion, we observed an overall increase in Ifng transcript levels in the SVF of aGr1-treated mice, relative to their untreated counterparts. This is most likely due to the IFNy-producing $\mathrm{CD}^{+}{ }^{+} \mathrm{T}_{\mathrm{EM}}$ that accumulate in AT following MDSC depletion (Figure 5.15 b). Interestingly, this same transcriptional upregulation is not seen at the whole tissue level (Figure 5.20 b). However, the transcriptional profile in aGr1-treated mice, at the whole tissue level, did exhibit some subtle changes compared to the untreated mice, which hinted at the importance of adipose-resident MDSCs in maintaining immune homeostasis in the tissue. For instance, we observed a significant downregulation of arginase in MDSC-depleted mice compared to untreated controls (Figure 5.20 b), suggesting that the loss of MDSCs in this tissue triggered a change in the immune regulatory activity of other non-stromal AT cells, potentially even within the adipocytes themselves. These transcriptional changes we observed at the stromal cell and whole tissue level do not necessarily indicate that the absence of adipose-resident MDSCs has shifted the AT microenvironment towards or away from a more pro-inflammatory state. However, taken together with the alterations we found in the size of the CD4+ ${ }^{+}$EM population size and ATM surface marker expression, it is clear that there are vital components of a robust, healthy adipose tissue stromal cell compartment. 

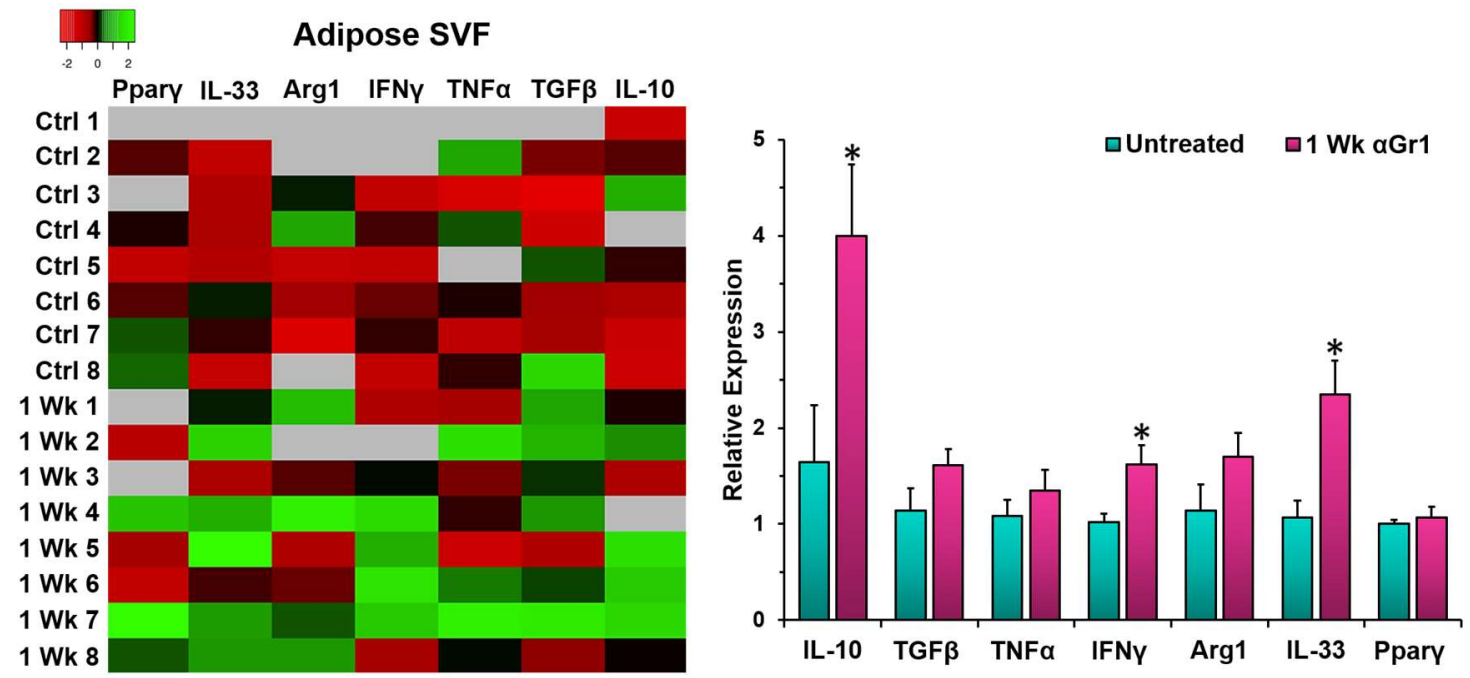

(b)

Whole Adipose Tissue

(d)
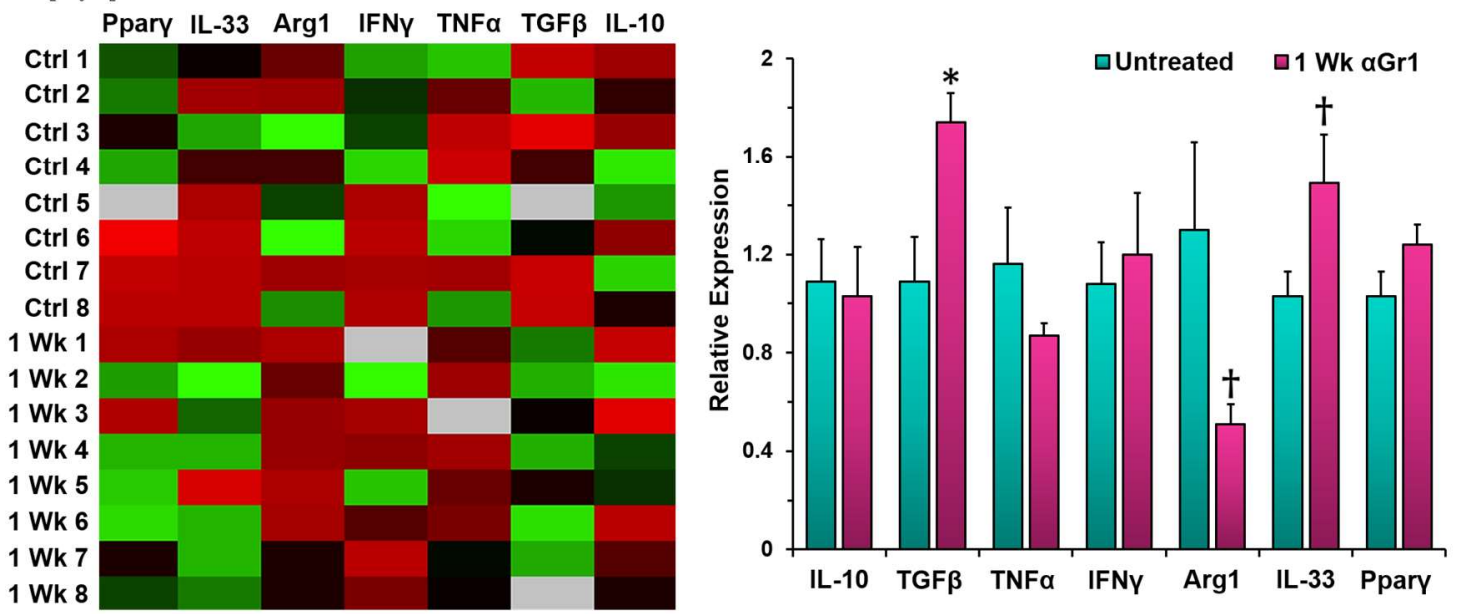

Figure 5.20: Adipose-resident MDSCs regulate whole tissue homeostasis as well as adipose immune cell dynamics. $(\mathbf{a}, \mathbf{b})$ Heatmap of gene expression in the SVF (a) or whole adipose tissue (b) isolated from aGr1-treated (1 Wk \#) or untreated (Ctrl \#) mice. Differential expression determined relative to mean $\Delta \Delta \mathrm{C}_{\mathrm{T}}$ of the untreated controls. Each row depicts expression of the specified gene from an individual animal. Grey boxes indicate outliers excluded from differential expression analysis. (c,d) Relative expression of specified genes in the SVF (c) or whole adipose tissue (d) of untreated (Blue) or aGr1-treated (Pink) mice. Bars represent the mean of 8 individual mice whose tissue and cells were processed independently. Error bars depict SEM. Significant differences between the two groups determined using Student's $t$-test. ${ }^{*}: P<0.05$ and $\dagger$ : $P<0.06$. 


\section{Conclusions}

We believe that we have demonstrated that adipose-resident MDSCs are an indispensable member of the immune cell network involved in maintaining AT homeostasis. In the absence of these cells the AT resident effector memory T cells begin to proliferate showing signs of increased activation with their upregulation of CD69. In addition to controlling the size of the adipose tissue TEM population, we have evidence that MDSCs have a moderate influence over ATM phenotype. In future work it will be interesting to see whether these surface marker alterations reflect a larger shift is ATM function as well.

We chose to focus on the macrophages and accumulated $\mathrm{CD} 4^{+} \mathrm{T}$ cells in healthy AT, as the phenotypes and functions of their tumor-associated counterparts are greatly influenced by MDSCs $[98,158,159]$. The increased number of $\mathrm{CD} 4^{+} \mathrm{TEMS}_{\mathrm{EM}}$, along with the altered expression of "M1" and "M2" surface markers on ATMs could suggest that the tissue microenvironment had become more "pro-inflammatory" as similar changes in cell phenotype and function are well-known hallmarks of the immune dysregulation and inflammation characteristic of obesity [154, 183-185]. However, the functionality of these two cell types, as assessed by cytokine expression, suggests otherwise. Effector cytokine and enzyme transcription in both ATMs and CD4+ TeMs was unaffected by a MDSC depletion.

Additionally, we did not see any conspicuous changes in the inflammatory status of AT in these mice. This result was not totally unexpected however, given that we were treating healthy animals that were not challenged in any other fashion besides the antibody depletion. ATMs and AT TEMS are just two of the many diverse 
types of cells that make-up the immune cell network mitigating inflammation in this tissue $[154,171,183]$. It is likely that they, along with these remaining members of the network, actively work to counteract some of the pro-inflammatory effects caused by MDSC depletion. Indeed, there was a significant transcriptional upregulation of immunosuppressive molecules such as IL-10, TGF $\beta$, IL-33, and arginase in the SVF and whole AT of Gr1-treated mice, despite expression levels of these mediators remaining unchanged in the sorted ATMs and CD4+ ${ }^{+}$EMS.

We did observe some changes in the tissue, that suggests that these compensatory responses were not sufficient to re-establish immune homeostasis, as $\mathrm{CD}^{+} \mathrm{T}_{\mathrm{EM}}$ accumulated and Ifng transcription increased. Additionally, we observed a moderate negative correlation between the number of adiposeresident MDSCs and the adiposity of that individual (Figure $5.8 \mathrm{~d}$ ), suggesting that the loss of MDSCs increases adipocyte lipid burden. Given the wide assortment of suppressive effector mechanisms at their disposal [100], MDSCs may modulate the function of one or more of the other AT-resident immune cells. The disruption of these unidentified interactions by $\alpha \mathrm{Gr} 1$ treatment and the subsequent downstream effects of these missing components of the cell network may account for the concomitant pro- and anti-inflammatory responses in the tissue.

Although adipose-resident MDSCs do not appear to be master regulators of immune homeostasis in the tissue, it is clear that AT immune dynamics are significantly dysregulated without the AT-resident MDSCs. Their ability to influence the immune activities of significantly larger cell populations is not unique among immunomodulatory cell types with as few as $30,000 \mathrm{CD} 8^{+}$"facilitating cells" reliably 
able to promote the successful engraftment of hematopoietic stem cells in an allogeneic recipient [186]. Additionally, type 2 innate lymphoid cells make up only about $1 \%$ of the AT SVF cells, yet their depletion has significant negative effects the composition of the healthy adipose tissue immune cell compartment $[30,31$, 187]. We propose that aMDSCs are essential players in the complex immune cell network in healthy AT, actively preventing the accumulation of $C D 4^{+} \mathrm{TEM}_{\mathrm{E}}$ in this tissue and, to a lesser extent, contributing to the stability of the "M2"-like nature of adipose-resident macrophages (ATMs), since their depletion leads to increased "M1"-like ATM, increased CD11C and MHC-II expression, and reduced CD301 expression. 


\section{CHAPTER 6: DISCUSSION \& FUTURE DIRECTIONS}

\section{MDSCs as Part of the Adipose Tissue Immune Cell Network}

Adipose is a dynamic tissue that relies on a diverse network of stromal (both immune and non-immune) cells to resolve metabolic and tissue remodeling related inflammation efficiently. With this project we characterized a novel population of adipose-resident MDSCs (aMDSCs) and gained some insight as to their place within the greater immunomodulatory cell network of adipose. Based on the findings, we propose that aMDSCs are an indispensable part of the AT immune compartment in healthy $\mathrm{AT}$, actively preventing $\mathrm{CD} 4^{+} \mathrm{TEM}_{\mathrm{EM}}$ proliferation in this tissue while also contributing to the stability of the "M2"-like nature of adipose-resident macrophages (ATMs) (Figure 6.1).

Our findings suggest that, once in the tissue, aMDSCs integrate seamlessly into the homeostatic immune cell network. For instance, we found that, compared to untreated controls, ATMs downregulate IL-10 transcription (Figure 5.19) and upregulate surface expression of CD11C and MHC-II (Figure 5.17), after acute MDSC depletion. Moreover, we found that aMDSCs are actively transcribing IL-10 (Figure 4.3). This combination of factors is also found in the tumor microenvironment, where tumor-associated macrophages engage in constant crosstalk with MDSCs via an IL-10-based positive feedback loop [114]. Given that 
ATMs reduced expression of this cytokine in the absence of aMDSC signaling, it is likely that they interact in a similarly cooperative fashion.

We also uncovered a novel relationship between aMDSCs and adipose tissue $T$ cells. When we treated healthy mice with $\alpha \mathrm{Gr} 1$ we found that the number of both $\mathrm{CD} 4^{+} \mathrm{TEM}_{\mathrm{EM}}$ and $\mathrm{CD} 8^{+} \mathrm{T}$ cells increased in the adipose. These $\mathrm{T}$ cells expressed elevated levels of CD69 on their surface and a greater percentage of them were $\mathrm{Ki} 67^{+}$compared to untreated controls. Once again this is similar to what others have demonstrated in the tumor microenvironment regarding MDSC and T cell dynamics $[177,178]$.

\section{T Cell Dynamics}

Our findings suggest that, in conjunction with other adipose stromal cells, the presence of aMDSCs in the tissue can further temper proliferative responses by adipose resident $T$ cells. While this is intriguing in and of itself, it also raises a key question about what is triggering these responses to begin with. At first glance the adipose-resident $T$ cell reaction to MDSC depletion seems counterintuitive given that a foreign antigen or inflammatory stimulus is typically required to incite proliferation and neither are present in healthy adipose tissue. However over the last decade many have found evidence which strongly implicates an, as of yet, unidentified self-antigen as the culprit behind T cell proliferation in healthy adipose [142]. One such study demonstrated that $\mathrm{CD} 4^{+} \mathrm{CD} 44^{+} \mathrm{CD} 62 \mathrm{~L}^{-}$effector memory $\mathrm{T}$ cells were present in the adipose tissue of healthy mice under normal dietary conditions [39], suggesting that $T$ cells had previously encountered and mounted a response against some adipose-derived antigen. 
In a broader sense, low-level, self-antigen-induced $\mathrm{T}$ cell proliferation has also been described before. Adoptively transferred naïve $T$ cells can reconstitute the entire T cell compartment in lymphopenic mice [188]. As they circulate these cells begin to proliferate, eventually repopulating both central and peripheral lymphatic tissues. Two distinct processes known as homeostatic and spontaneous proliferation are behind this phenomenon [189, 190]. Homeostatic proliferation occurs at a slower rate than canonical, foreign-antigen induced proliferation and relies on the availability of cytokines such as IL-7 and IL-15 [189, 191]. Competition for these resources is thought to be the signal that determines when the $T$ cell compartment within a particular niche is sufficiently repopulated [188].

Spontaneous proliferation, on the other hand, does not require any secondary cytokine signal. This type of proliferation occurs at a much faster rate than homeostatic proliferation, with cells dividing multiple times a day instead of just once or twice every few days [190,192]. The resulting T cells also differentiate into various subsets of effector and memory $\mathrm{T}$ cells, capable of producing inflammatory cytokines $[40,190]$.

Despite these major differences, antigen presentation and recognition are required for both homeostatic and spontaneous $T$ cell proliferation [188]. Reconstitution studies in both Rag knockout and germ-free mice indicate that these processes are likely initiated by low affinity interactions with self-antigen [191], presented by MHC-II on antigen presenting cells. Intriguingly, this bears a striking resemblance to what occurs in the early stages of obesity, where resident macrophages and dendritic cells present an unknown self-antigen to tissue 
resident $\mathrm{CD} 4^{+} \mathrm{TEM}_{\mathrm{EM}}$, triggering a cascade of events that eventually leads to chronic AT inflammation [193]. In this situation however, the high fat diet itself serves as an additional inflammatory stimulus, augmenting the proliferative response of the T cells. It is possible that in our MDSC depletion model, the T cells are responding to this same self-antigen, but, in the absence of any other inflammatory pressure, the size of their population is held in check by mechanisms similar to those which control T cell expansion in models of homeostatic and spontaneous proliferation. Regardless, these examples of non-foreign, antigen-induced $\mathrm{T}$ cell proliferation demonstrate that T cell biology is far more nuanced than is often assumed.

\section{Macrophage Dynamics}

Besides controlling the T cell population and modulating ATM phenotype, adipose resident MDSCs likely have beneficial interactions with other cells in the tissue. To this end, we examined the expression of immunomodulatory genes in whole adipose tissue and within the heterogeneous SVF isolate after acute MDSC

depletion. We discovered transcriptional changes (Figure 5.20) that, in some cases, were unexpected given that expression of these same genes had not been affected on the individual cell level in MDSCs, ATMs, and CD4 ${ }^{+} \mathrm{TEM}_{\mathrm{E}}$ cells (Figure

\section{$5.15 \& 5.19)$.}

Importantly, our results do not indicate that aMDSCs are the ultimate regulator of immune homeostasis in healthy adipose tissue. Instead they paint a much more nuanced picture where the diverse immune cell network in AT is constantly monitoring and responding to the dynamic challenges presented by this metabolically active tissue. In this proposed working model, the loss of aMDSCs 
does not have catastrophic consequences, rather it destabilizes the entire network, which then adjusts to counteract pro-inflammatory effects. Although this does restore a semblance of normalcy to the tissue, there is evidence that this loss has a lasting impact on the tissue, as we see with the continued increase in MHC-II expression by ATMs and the progressive accumulation of CD4 ${ }^{+} \mathrm{TEMS}_{\mathrm{E}}$ in the tissue, despite there being a partial restoration of the aMDSC population.

Additionally, we observed a moderate negative correlation between the number of adipose-resident MDSCs and the adiposity of that individual (Figure 5.8) suggesting that the loss of aMDSCs increases adipocyte lipid burden. Since the animals in this study were only treated for one week, it is tempting to speculate that long-term MDSC depletion may lead to increased weight gain on a normal diet, when compared to untreated individuals. Furthermore, the alterations we observed in the AT of aGr1-treated mice could exacerbate and accelerate the development of metabolic consequences induced by high fat diet as these symptoms are often preceded by macrophage repolarization and the infiltration of activated T cells into the tissue [141]. A recent report found that MDSC depletion, concurrent with 8 weeks of high fat diet, increased the severity of diet-induced metabolic symptoms as well as TNFa transcription in the tissue compared to high fat diet alone [194]. In our study, CD4+ TEM accumulated in the tissue of otherwise healthy mice following MDSC depletion. If the Gr1 treatment used in this high fat diet study elicited a similar response, then it's possible that the heightened metabolic symptoms they observed in treated animals were at least partially due to this augmented, initial T cell presence. Taken together with the results of our 
study, this suggests that the immune dysregulation we observed in healthy AT, induced by MDSC depletion, may predispose this tissue to increased inflammatory and metabolic consequences when challenged with a high fat diet. 


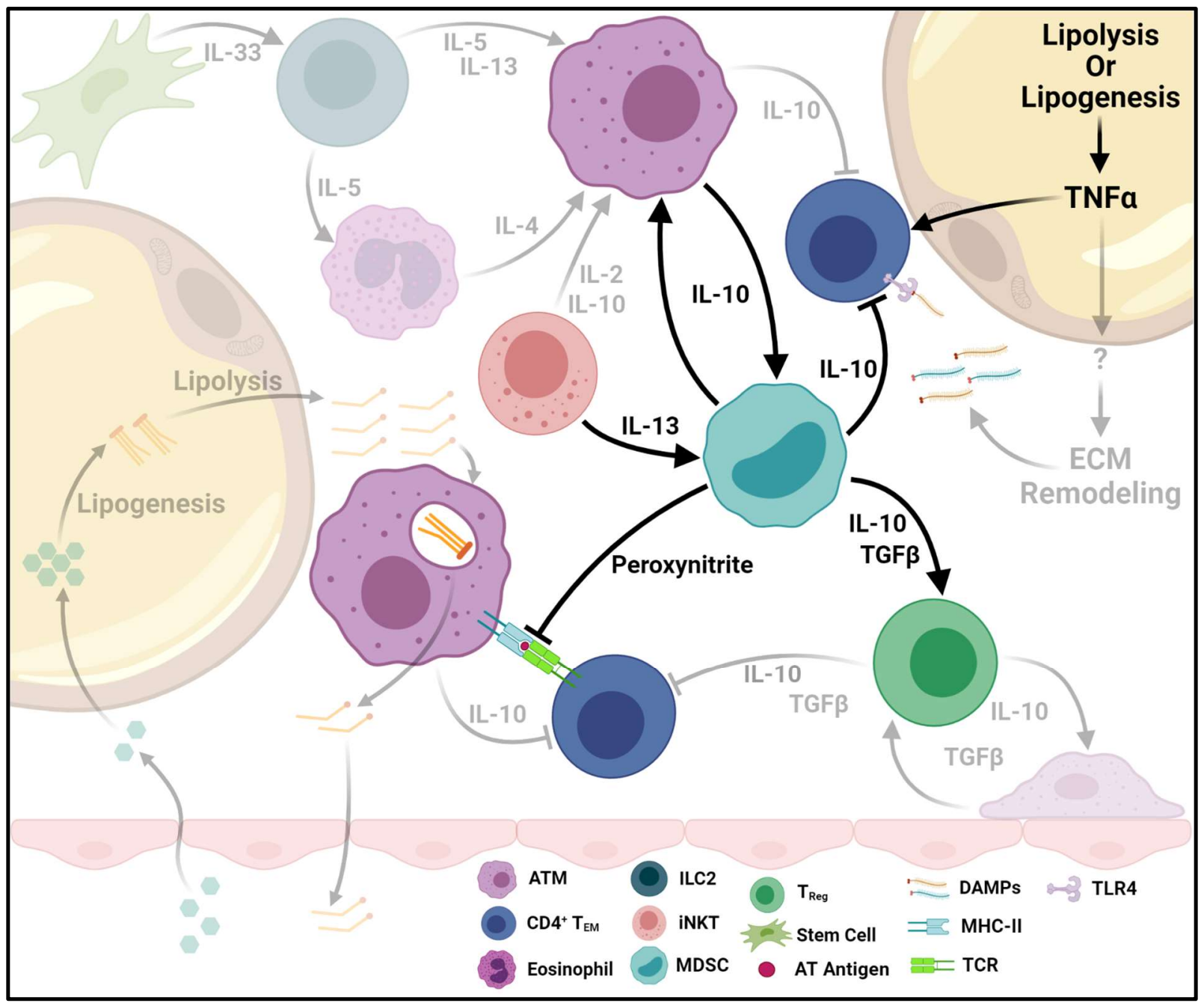

Figure 6.1: The Role of aMDSCs in Healthy Adipose Tissue. aMDSCs prevent effector memory $\mathrm{T}$ cell proliferation ( $\mathrm{T}_{\mathrm{EM}}$ ) and promote the suppressive "M2" phenotype of adipose tissue-resident macrophages (ATMs) in healthy adipose tissue. 


\section{Caveats to the aGr1 Model of MDSC Depletion}

To investigate the importance of MDSCs in the homeostatic health of AT, we employed the classic aGr1 antibody-mediated model of in vivo MDSC depletion. This specific model and antibody clone have long been used to interrogate the impact of MDSCs in various pathological settings. As discussed in Chapter 5, Gr1 is expressed on multiple cell types; however, we were relatively confident that the effects we observed in healthy adipose following antibody treatment were instigated specifically by the loss of MDSCs and not the depletion of other known cellular targets of this antibody such as neutrophils and some subsets of $\mathrm{CD}^{+} \mathrm{T}$ cells (Figure 5.1). However, a recent study found that eosinophils in an IL-5 rich microenvironment upregulated their surface expression of Gr1 (as detected by the RB6-8C5 antibody clone) and Ly6G (as detected by the 1A8 antibody clone) [195]. Given that adipose resident eosinophils are recruited into the tissue via IL-5 produced by ILC2s it's likely that they also exhibit this elevated $\mathrm{Gr} 1 / \mathrm{Ly} 6 \mathrm{G}$ expression. If this is the case, then it is possible that our antibody treatment may have reduced or depleted eosinophils resident in adipose. Their loss within the tissue could offer an alternate explanation for the aGr1induced changes we observe following acute treatment, since eosinophils are known to promote the "M2"-like nature of ATMs [35]. The inadvertent depletion of adipose eosinophils would not however lead to the rapid accumulation of CD4 ${ }^{+}$ $\mathrm{T}_{\mathrm{EM}}$ that we observe in both acutely and chronically treated mice.

One of our more perplexing observations was the partial restoration of MDSCs in the spleens and adipose of mice treated for 4 weeks with aGr1. After verifying that the antibody was not defective and switching to a weight-based 
dosing system, there seemed to be no clear explanation for the incomplete depletion we were seeing in the 4 week treated mice. Looking back at the literature we soon discovered that in most studies employing this depletion model animals only receive one or two injections over a period ranging from 24 hours to 1 week. Of the studies that used the antibody for more than a week [196-198], none reported the reappearance of the $\mathrm{Gr} 1^{+}$cells they were targeting. In fact, when they reported the efficacy of their MDSC depletion, if they did at all, they described almost complete ablation of the $\mathrm{Gr} 1^{+}$population. Digging into the details of their methodology, I uncovered a common flaw in the verification methods used in both acute and chronic aGr1-mediated depletion studies. Often, investigators treat animals with the RB6-8C5 clone of the aGr1 antibody, stain collected tissues with a fluorophore conjugated version of this same clone, then declare that depletion was successful when they don't find any $\mathrm{Gr} 1^{+}$cells. This however fails to account for epitope masking caused by the Gr1 treatment. Ideally the dose used to treat the mice should be high enough to evenly distribute the antibody throughout their tissues to reach as many of their Gr1 positive cells as possible. If this is the case, then the surface of these cells should be saturated with antibody, making the surface protein unavailable for binding by additional $\mathrm{Gr} 1$ antibodies used to stain the tissue. Subsequent analysis via flow cytometry or microscopy would likely underrepresent the true number of surviving MDSCs. Others used the $1 \mathrm{~A} 8$ clone of the Ly6G antibody to verify depletion [196, 198], however, previous staining or treatment with $\alpha \mathrm{Gr} 1$ is known to block the Ly6G binding site. 
A small contingent of investigators have started to call for reform. In 2016 the Journal of Leukocyte Biology published a paper demonstrating that continued treatment with the $\alpha \mathrm{Gr} 1$ antibody for more than 10 days appeared to induce emergency myelopoiesis and severely diminished the antibody's efficacy [170]. This "Ly-6G rebound" only became apparent when they stained tissues and cells with a fluorophore conjugated secondary antibody against rat lgG2b, allowing them to visualize $\mathrm{Gr} 1^{+}$cells without using the $\mathrm{Gr} 1$ antibody. An editorial published in the same issue called for a reassessment of the long-term use of this model as well as the ways in which we verify it $[168,170]$. These warnings seem to have fallen on deaf ears however as studies that verify Gr1-mediated depletion by staining for $\mathrm{Gr} 1^{+}$cells continue to be published in journals at all levels.

We believe that the resurgence of MDSCs that we see in our 4 week treated mice was not an artifact of insufficient treatment administration nor was it the result of a defective $\alpha \mathrm{Gr} 1$ antibody, since we used a secondary, non-Gr1-associated phenotype to characterize and quantify these cells (CD11 $b^{H i} \mathrm{Ly}_{6 \mathrm{C}^{\mathrm{Hi}}} \mathrm{CD}^{\mathrm{N}} 1 \mathrm{c}^{-}$ SSC Low). Additionally, our findings corroborate the "Ly-6G rebound effect" described by Moses et al (2016), further supporting the need to carefully reexamine how the $\alpha \mathrm{Gr} 1$ depletion model was used in the past and reassess how it should be used in future studies.

\section{Sexual Dimorphism in Healthy Adipose Immune Homeostasis}

Like the majority of studies on the adipose tissue immune cell compartment we almost exclusively collected data from male mice. However, there is a growing body of literature that is beginning to reveal that anatomically equivalent adipose 
depots may behave differently in males and females [199-203]. For instance, there is evidence that the immune "programming" in females is generally more suppressive than in males $[201,203]$. In the realm of adipose biology this means that gonadal adipose depots tend to be less inflamed in female mice compared to males when they are fed a high fat diet, containing fewer CD11 $\mathrm{c}^{+}$ATMs and expressing lower levels of proinflammatory genes such as II1b and Nos2 [199]. This along with the differences we observed between males and females in our pilot $\alpha \mathrm{Gr} 1$ depletion experiments (Figure 5.2) motivated our initial decision to analyze adipose from both male and female mice. Although time restraints eventually forced us to focus our investigations on males alone, we did obtain partial datasets from both acute $(\mathrm{N}=4)$ and chronic $(\mathrm{N}=6)$ treatment groups, as well as a small cohort of untreated controls $(\mathrm{N}=3)$.

Confirming what we observed in our pilot experiments, acute aGr1 treatment effectively eradicated MDSCs in the adipose of female mice $[12.75 \pm 2.7$ x $10^{3} \mathrm{aMDSCs} / \mathrm{mL}$ AT vs $0.44 \pm 0.05 \times 10^{3} \mathrm{aMDSCs} / \mathrm{mL}$ AT] (Figure 6.2). However, this population had completely rebounded in the adipose of chronically treated mice $\left[19.98 \pm 2.5 \times 10^{3} \mathrm{aMDSC} / \mathrm{mL} \mathrm{AT]}\right.$ (Figure 6.2).

The frequency $[1.5 \pm 0.3 \%$ of AT SVF vs $2.23 \pm 0.3 \%$ of AT SVF] and absolute number $\left[4.53 \pm 1.3 \times 10^{4} \mathrm{CD}^{+} \mathrm{T}\right.$ cells $/ \mathrm{mL}$ AT vs $7.37 \pm 1.3 \times 10^{4} \mathrm{CD}^{+} \mathrm{T}$ cells/mL AT] of $\mathrm{CD} 4^{+} \mathrm{T}$ cells increased steadily over time (Figure 6.3 a), eventually reaching statistical significance after 4 weeks of $\alpha \mathrm{Gr} 1$ treatment $[2.84 \pm 0.2 \%$ of AT SVF; $11.21 \pm 1.3 \times 10^{4} \mathrm{CD}^{+} \mathrm{T}$ cells/mL AT], despite the MDSC population being almost completely restored (Figure 6.3 a). It is likely that the acute treatment 
group would have reached statistical significance given a larger cohort of untreated controls as adipose isolated from untreated males in this same experiment contained an unusually high number of T cells, potentially due to the stress from a recent room transfer within the animal housing facility. Similar to what we found in males the majority of these cells belonged to the $\mathrm{CD} 44^{+} \mathrm{CD} 62 \mathrm{~L}^{-}$effector memory subset of T cell (Data not shown). Unlike the males however CD69 expression on these cells was unaffected by MDSC depletion (Figure 6.3 b).

Adipose from untreated female mice contained an almost negligible population of $\mathrm{CD} 8^{+} \mathrm{T}$ cells $\left[66 \pm 50 \mathrm{CD}^{+} \mathrm{T}\right.$ cells $\left./ \mathrm{mL} \mathrm{AT}\right]$ which expanded in response to MDSC depletion [1 Week: $832 \pm 300 \mathrm{CD} 8^{+} \mathrm{T}$ cells/mL AT; 4 Week: 948 $\pm 230 \mathrm{CD}^{+} \mathrm{T}$ cells $/ \mathrm{mL}$ AT] (Figure $6.3 \mathrm{c}$ ). The variation within each cohort however is very large and the proportions of $\mathrm{CD} 8^{+} \mathrm{T}$ cells is relatively small, so it is difficult to draw any reliable conclusions from this data.

We also examined the SVF from female mice for any charges in the resident macrophage population. We found that acute and chronic MDSC depletion induced a steady increase in the proportion [Untreated: $6.62 \pm 0.8 \%$ of AT SVF; Acute: $8.52 \pm 0.9 \%$ of AT SVF; Chronic: $10.9 \pm 0.8 \%$ of AT SVF] and absolute number [Untreated: $21.3 \pm 6.5 \times 10^{4}$ ATMs $/ \mathrm{mL}$ AT; Acute: $27.5 \pm 2.9 \times 10^{4}$ ATMs $/ \mathrm{mL}$ AT; Chronic: $43.8 \pm 6.2 \times 10^{4}$ ATMs $/ \mathrm{mL} \mathrm{AT]} \mathrm{of} \mathrm{ATMs} \mathrm{(Figure} 6.4$ a). Both CD11C and MHC-II expression spiked following 1 week of treatment with aGr1 [CD11c: $1.0 \pm 0.004$ vs $1.27 \pm 0.04 ;$ MHC-II: $1.0 \pm 0.05$ vs $1.49 \pm 0.1]$. While the relative MFI of these two surface markers drops slightly on the ATMs from 4- 
week treated females it does remain elevated compared to ATMs isolated from untreated mice [CD11c: $1.12 \pm 0.02$; MHC-II: $1.33 \pm 0.09$ ] (Figure 6.4 b).

Although this dataset is incomplete, it does highlight some intriguing differences between the adipose immune cell network in males and females. For instance, the MDSC rebound in adipose of chronically treated females appears to be more robust than what we observed in the males $[156.7 \%$ vs $60.4 \%$ of respective untreated aMDSC population]. A study looking at sexually dimorphic MDSC responses in viral myocarditis found that females recruited a greater number of M-MDSCs into the cardiac tissue than males and that these MDSCs demonstrated superior suppressive abilities compared to the equivalent population isolated from males [204]. One of the proposed mechanisms for the "Ly6G rebound" is that the aGr1 antibody induces emergency myelopoiesis, a process that can also recruit MDSCs to the site of chronic inflammation.

MDSC levels increase in both males and females in the myocarditis model. However, females were able to more efficiently recruit highly suppressive MDSCs into the inflamed tissue than males, which ultimately improved their survival over males. It's possible that we are seeing a similar differential response in the adipose of male and female mice with the females demonstrating their ability to more efficiently recruit MDSCs under myelopenic pressure.

Without functional or gene expression data we cannot really draw any solid conclusions much beyond that there does appear to be a sexually dimorphic response to MDSC depletion in otherwise healthy adipose tissue. Given our limited picture it is tempting to speculate that the slower rate of $\mathrm{T}$ cell accumulation, 
reduced CD69 expression, and expanding ATM population we observed in females are all related to their increased capacity to prevent visceral adipose inflammation compared to males. 
(a)

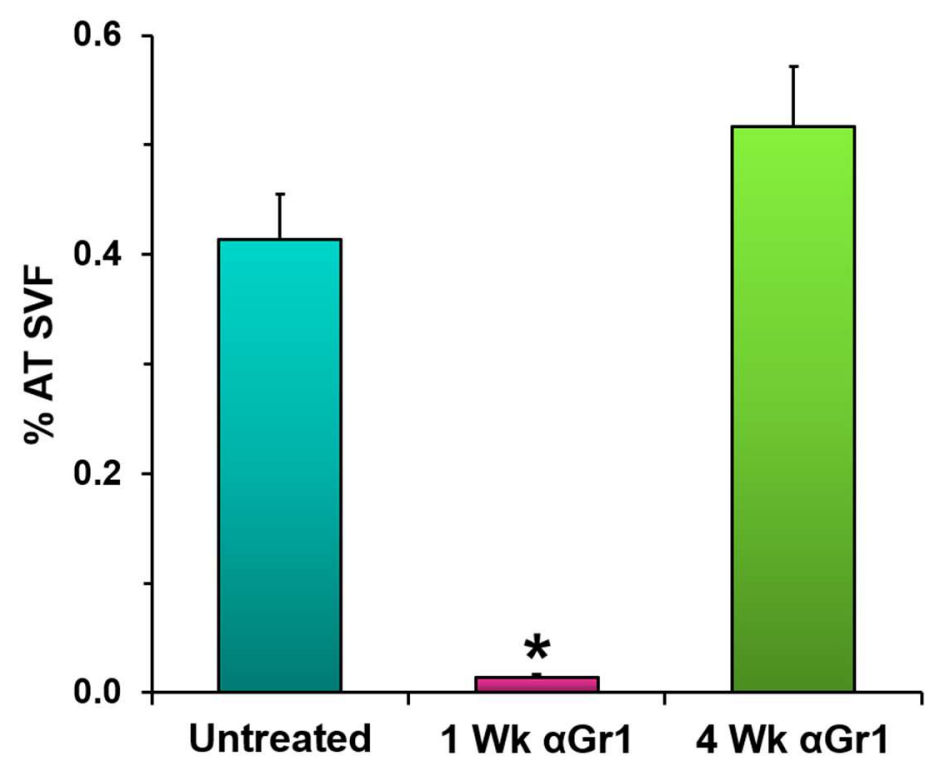

(b)

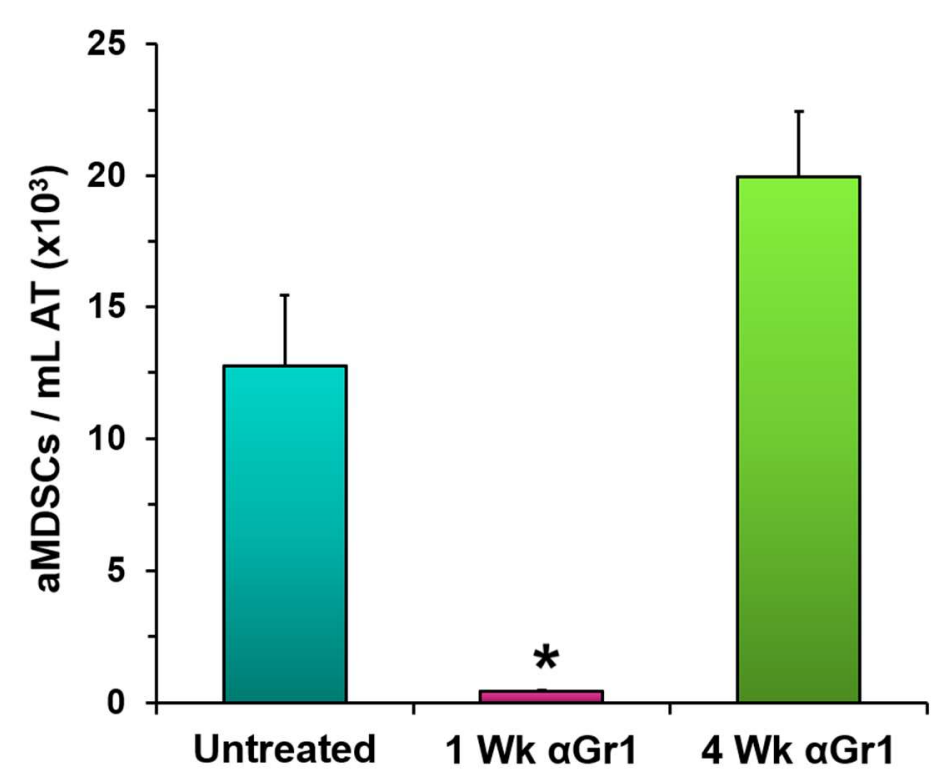

Figure 6.2: $\alpha \mathrm{Gr} 1$ antibody treatment efficiently depletes MDSCs from adipose tissue in female mice. (a, b) Healthy, 16 - 20-week old female, C57BI/6 mice were treated with $\alpha \mathrm{Gr} 1(15 \mu \mathrm{g} / \mathrm{g})$ every $3-4$ days for 1 or 4 weeks. Uterine horn fat pads were collected one day after the final antibody injection. The frequency (a) and absolute number (b) of aMDSCs (M-MDSCs + PMN-MDSCs) in AT SVF from untreated (Blue) mice or animals receiving 1-week (Pink) or 4-weeks of $\alpha \mathrm{Gr} 1$ treatment (Green). Bars depict the mean of 3 untreated, 4 1-week aGr1-treated, or 64 -week aGr1-treated mice. Differences between aGr1-treated and untreated animals determined using a Student's $t$-test. *: $P<0.05$. 
(a)

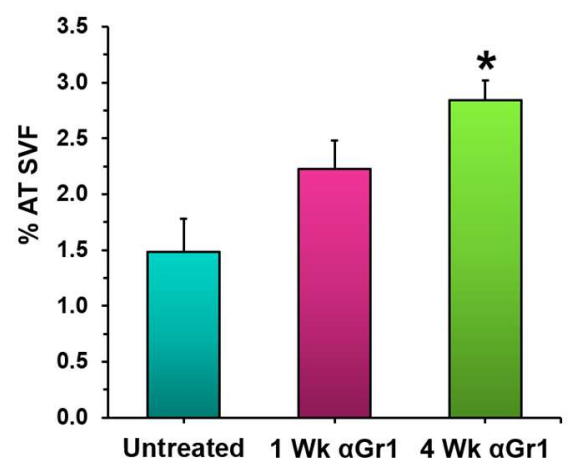

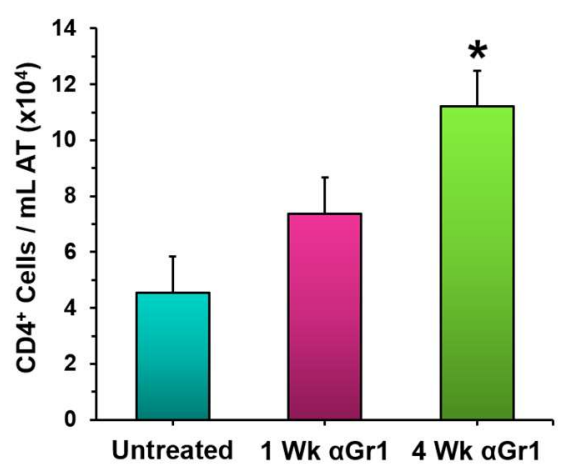

(b)

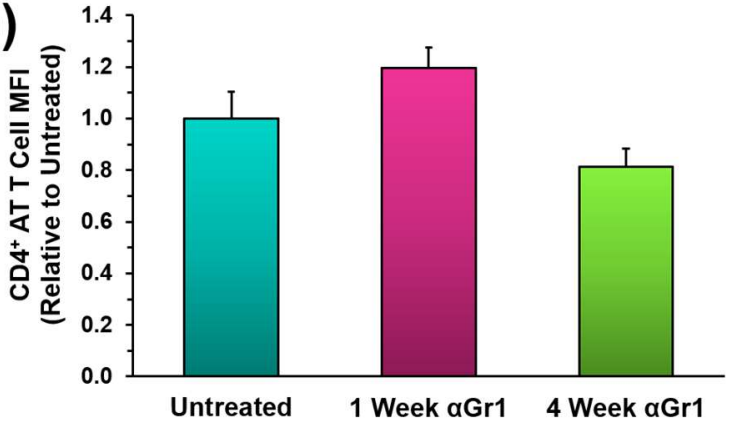

(c)
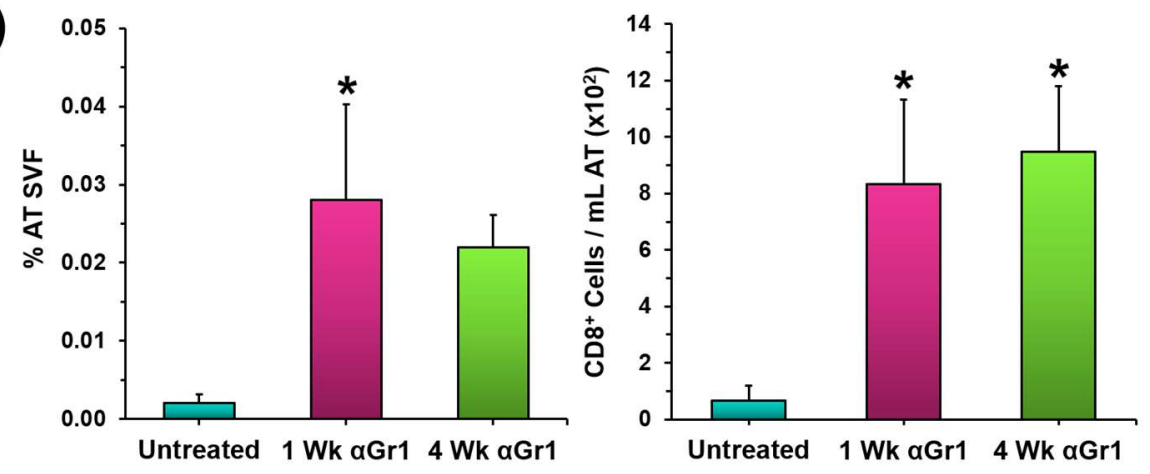

Figure 6.3: MDSC depletion increases the numbers of $\mathrm{T}$ cells in healthy female adipose tissue. Healthy, 16 - 20-week old female C57BI/6 mice were treated with aGr1 $(15 \mu \mathrm{g} / \mathrm{g})$ every $3-4$ days for 1 or 4 weeks. Uterine horn fat pads were collected one day after the final antibody injection. (a) Frequency (Left) and absolute number (Right) of $\mathrm{CD}^{+} \mathrm{T}$ cells in the SVF of aGr1-treated and untreated mice. (b) The geometric mean fluorescence intensity (MFI) of CD69 expression on CD44+ CD62L- TEM in the SVF of either 1-week (Pink) or 4-week (Green) $\alpha \mathrm{Gr} 1$-treated mice relative to untreated controls (Blue). (c) Frequency (Left) and absolute number (Right) of CD8 ${ }^{+} \mathrm{T}$ cells in the SVF of aGr1-treated and untreated mice. Bars depict the mean of 3 untreated (Blue), 4 1-week aGr1-treated (Pink), or 6 4-week aGr1-treated mice (Green). (a - c) Data pooled from 3 independent experiments. Error bars represent SEM. Differences between aGr1treated and untreated animals determined using a Student's $t$-test. *: $P<0.05$ and NS: $P>0.05$. 

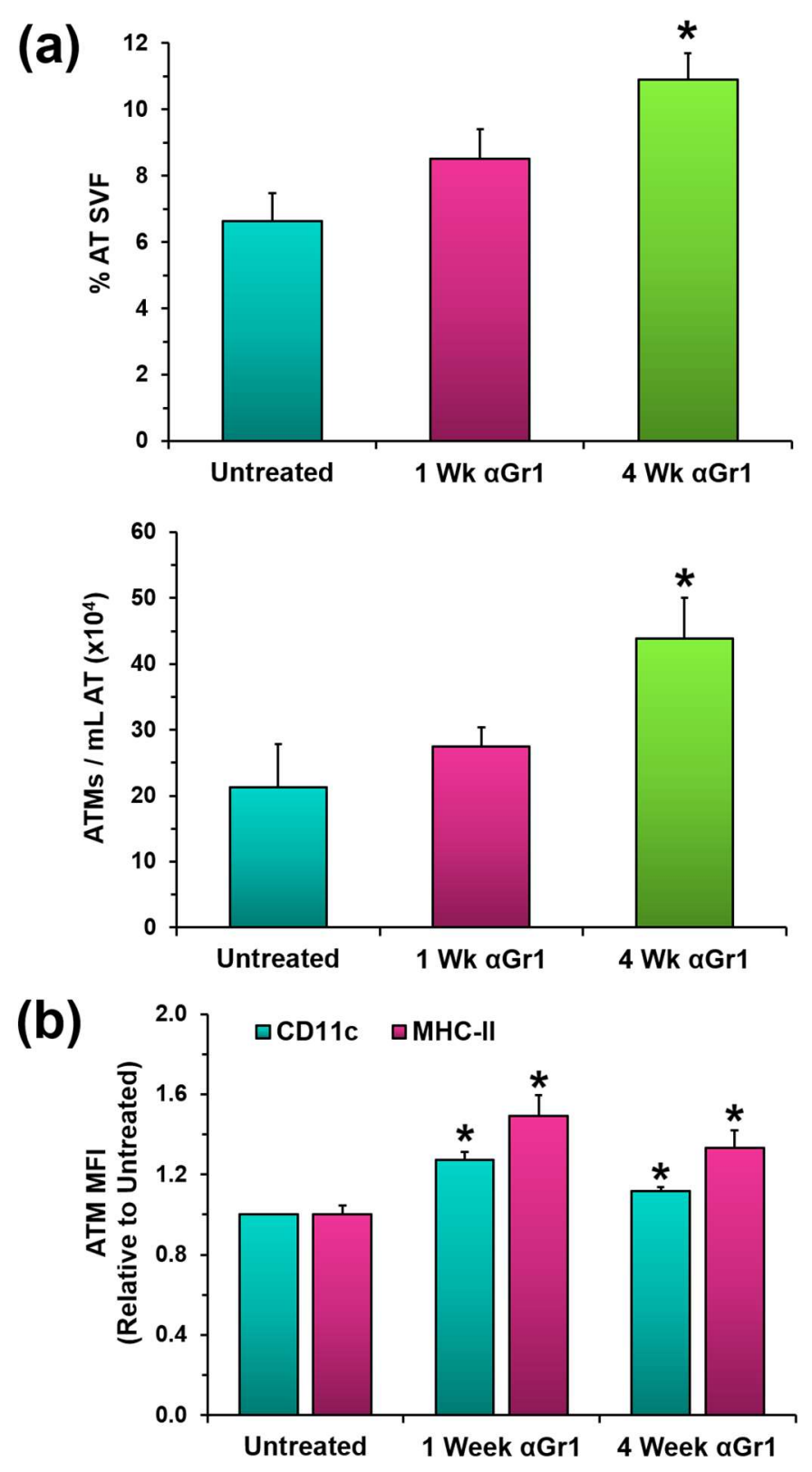

Figure 6.4: $\alpha \mathrm{Gr} 1$ treatment augments the number of macrophages \& changes their surface marker expression in female adipose tissue. Healthy, $16-20$ week old female C57BI/6 mice were treated with aGr1 (15 $\mu \mathrm{g} / \mathrm{g})$ every $3-4$ days for 1 or 4 weeks. Uterine horn fat pads were collected one day after the final antibody injection. (a) Frequency (Upper) and absolute number (Lower) of CD11 b ${ }^{+}$ $\mathrm{F} 4 / 80^{+} \mathrm{MHC}-\mathrm{II}^{+}$adipose tissue-resident macrophages (ATMs) in the total AT SVF. (b) The geometric mean fluorescence intensity (MFI) of CD11c (Blue) \& MHC-II (Pink) on $\mathrm{CD} 11 \mathrm{~b}^{+} \mathrm{F} 4 / 80^{+} \mathrm{MHC}-\mathrm{II}^{+}$ATMs following 1 or 4 weeks of $\alpha \mathrm{Gr} 1$ treatment. Data pooled from 3 independent experiments. Error bars represent SEM. Differences between $\alpha \mathrm{Gr} 1$-treated and untreated animals determined using a Student's $t$-test. ${ }^{*}: P<0.05$ and NS: $P>0.05$. 


\section{Translational \& Therapeutic Potential}

Recently, one group found that while the overall percentage of CD45 adipose stromal cells was similar in mice, non-human primates, and humans, the proportions of various immune cell subsets within that $\mathrm{CD} 45^{+}$population differed greatly from one species to the next [205]. Their work raised major concerns regarding the translatability of adipose biology studies from the bench into the clinic since the immune cell compartment in this tissue appeared to vary so widely even between different strains of mice. In our studies we addressed these concerns on two fronts. First, we sought to determine whether the presence of aMDSCs in healthy mice was consistent between strains or if it was an artifact of our specific model. To this end we performed a cursory analysis of the immune cell compartment in four male Balb/c mice using our established myeloid and T cell staining panels. Similar to the study referenced above, we found that there was no difference in the total number of adipose stromal cells $\left[22.7 \pm 2.0 \times 10^{5}\right.$ cells $/ \mathrm{mL}$ AT vs $24.7 \pm 2.4 \times 10^{5}$ cells $/ \mathrm{mL} \mathrm{AT]}$ between the two mouse strains (Figure 6.5 a). There was also no difference in the frequency $[0.575 \pm 0.06 \%$ of AT SVF vs 0.419 $\pm 0.06 \%$ of AT SVF] or absolute number $\left[1.57 \pm 0.2 \times 10^{4}\right.$ cells $/ \mathrm{mL}$ AT vs $1.69 \pm 0.7$ $x 10^{4}$ cells $/ \mathrm{mL} \mathrm{AT]}$ of aMDSCs in adipose from the C57BI/6 and Balb/c mice (Figure 6.5 b). The adipose from Balb/c mice tended to contain more CD301+ ATMs than C57BI/ 6 mice $\left[36.23 \pm 3.7 \times 10^{4}\right.$ cells $/ \mathrm{mL}$ AT vs $47.29 \pm 14.0 \times 10^{4}$ cells $/ \mathrm{mL} \mathrm{AT]}$, but this difference was not significant (Figure 6.5 b). However, CD4 ${ }^{+}$ T cells did make up a significantly larger proportion of the AT SVF from the Balb/c mice compared to the C57BI/6 mice $[1.82 \pm 0.4 \%$ of AT SVF vs $0.86 \pm 0.07 \%$ of 
AT SVF] and, intriguingly, a significantly smaller percentage of this population were $\mathrm{CD}_{4}{ }^{+} \mathrm{CD}^{2} \mathrm{~L}^{-} \mathrm{T}_{\mathrm{EM}}\left[64.4 \pm 5.8 \%\right.$ of $\mathrm{CD} 4^{+}$SVF cells vs $97.4 \pm 0.4 \%$ of $\mathrm{CD} 4^{+} \mathrm{SVF}$ cells] (Figure $6.5 \mathrm{c}-\mathrm{d}$ ).

Our second way of addressing the translatability of our work was by characterizing the stromal cells in samples of human adipose tissue. Samples were obtained from patients undergoing routine liposuction procedures and were processed in much the same way as our murine adipose samples. Cells were then stained and analyzed using flow cytometry. Despite being harvested from a subcutaneous, instead of visceral, adipose depot, we identified two myeloid cell populations that expressed surface markers characteristic of human M-MDSCs $\left(\mathrm{CD} 11 \mathrm{~b}^{+} \mathrm{CD}^{3} 3^{+} \mathrm{HLA}^{-D R^{-}}\right.$CD14 $\left.{ }^{+}\right)$and PMN-MDSCs (CD11b+ HLA-DR ${ }^{-}$CD14$\mathrm{CD}^{15^{+}}$) [100] (Figure $\left.6.6 \mathbf{a}-\mathbf{b}\right)$. Unfortunately, due to time and equipment access restrictions we were not able to perform the requisite in vitro functional assays with these cells and are therefore unable to draw any concrete conclusions regarding their identity as true MDSCs. However, adipose in some form or another is highly conserved evolutionarily and from the fat bodies found in insects to the various adipose depots in mammals, this metabolically dynamic tissue is consistently intertwined with the regulatory branch of the immune system $[185,206,207]$. As such it is likely that the two myeloid cell populations, which we identified in human adipose, are indeed immunosuppressive MDSCs, involved in moderating adipose immune homeostasis.

In addition to our characterization data, the findings from clinical trials of autologous SVF cellular therapies provide further evidence supporting the 
presence of MDSCs in human adipose. SVF-based therapies have a potent antiinflammatory effect when they are used to treat allogeneic transplant rejection. Many aspects of this response such as increased angiogenesis or enriched populations of $\mathrm{T}_{\text {Regs }}$ and "M2" polarized macrophages have also been described in clinical trials where in vitro generated MDSCs alone are used to treat rejection [91, $208,209]$. It is important to note however that the overall anti-inflammatory effects of SVF-based therapies most likely results from the combined efforts of all of the AT-resident immune cells, as their collective crosstalk preserves the suppressive phenotypes of each individual cell once they are introduced into a pro-inflammatory microenvironment.

Moreover, MDSCs resident in healthy human adipose tissue could represent a new therapeutic target for diabetes and obesity. These diseases are characterized by a disruption in the anti-inflammatory adipose microenvironment, mediated by the infiltration of inflammatory immune cells into the tissue. Studies have already demonstrated that injections of culture derived MDSCs into obese mice increased insulin sensitivity and delayed the onset of metabolic syndromes [129]. Determining whether MDSCs play a significant part in maintaining immune homeostasis in human AT could help us develop new therapies that designed to promote and re-establish homeostatic conditions in obese adipose where the immunosuppressive cell network has been significantly degraded. 
(a)

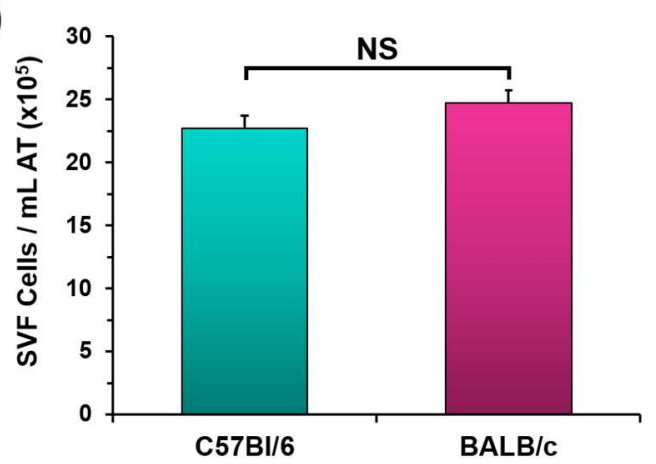

(c)

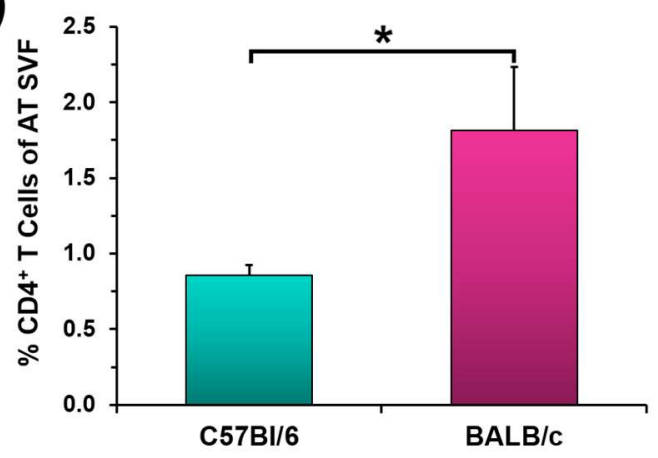

(d)

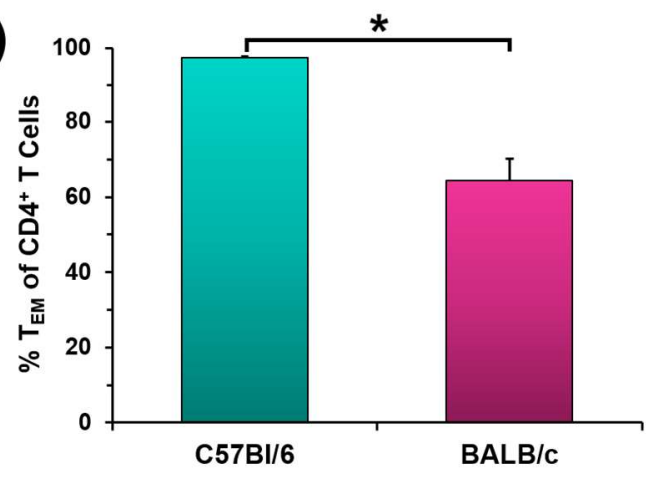

(b)
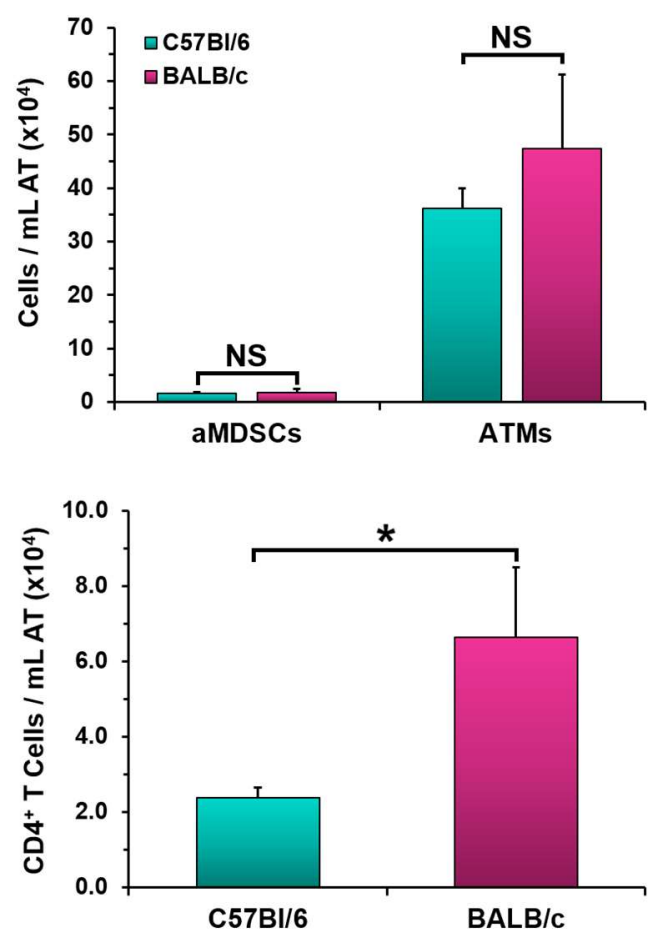

Figure 6.5: Flow cytometric characterization of healthy adipose tissue from Balb/c mice. (a) The absolute number of SVF cells obtained (normalized to the volume of adipose tissue harvested) from either C57BI/6 (Blue) or Balb/c (Pink) mice. (b) The absolute number of aMDSCs and $\mathrm{CD} 11 \mathrm{~b}^{+} \mathrm{F} 4 / 80^{+} \mathrm{MHC}-\mathrm{Il}^{+}$ macrophages (ATMs) in the adipose SVF of C57BI/6 (Blue) or Balb/c (Pink) mice. (c) The frequency (Left) and absolute number (Right) of $\mathrm{CD}^{+} \mathrm{T}$ cells in the SVF of aGr1-treated and untreated mice. (d) Frequency of CD4 ${ }^{+}$SVF cells that were $\mathrm{CD}_{4}{ }^{+}$CD62L- TEM. $(\mathbf{a}-\mathbf{d})$ Bars depict the mean of $11 \mathrm{C} 57 \mathrm{BI} / 6$ or $4 \mathrm{Balb} / \mathrm{c}$ mice. Error bars denote the SEM. Differences between the C57BI/6 and Balb/c mice determined using a Student's $t$-test. *: $P<0.05$ and NS: $P>0.05$. 
(a)

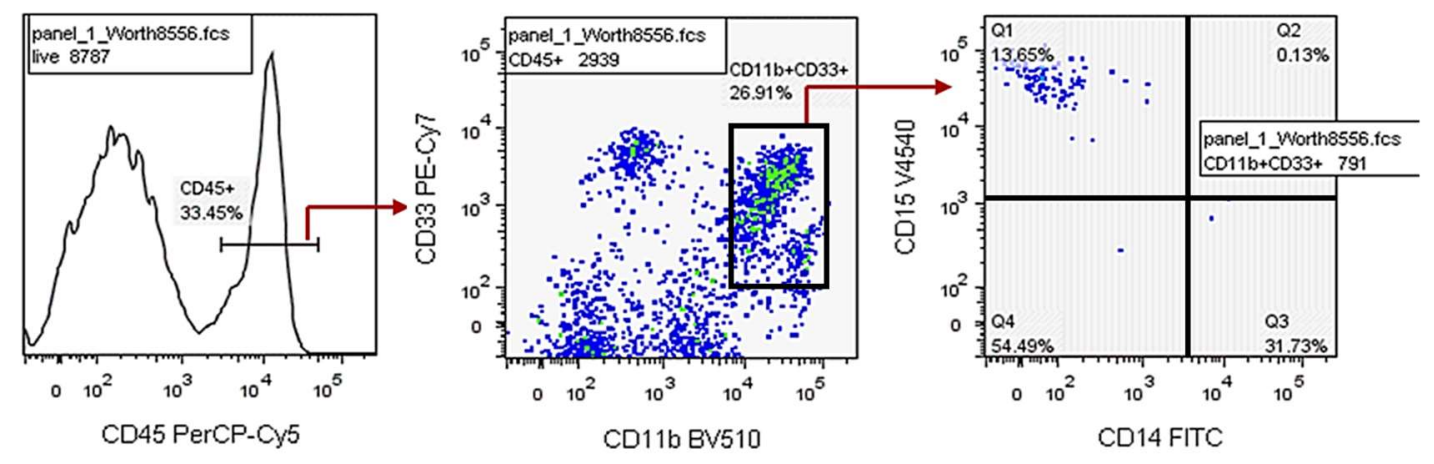

(b)

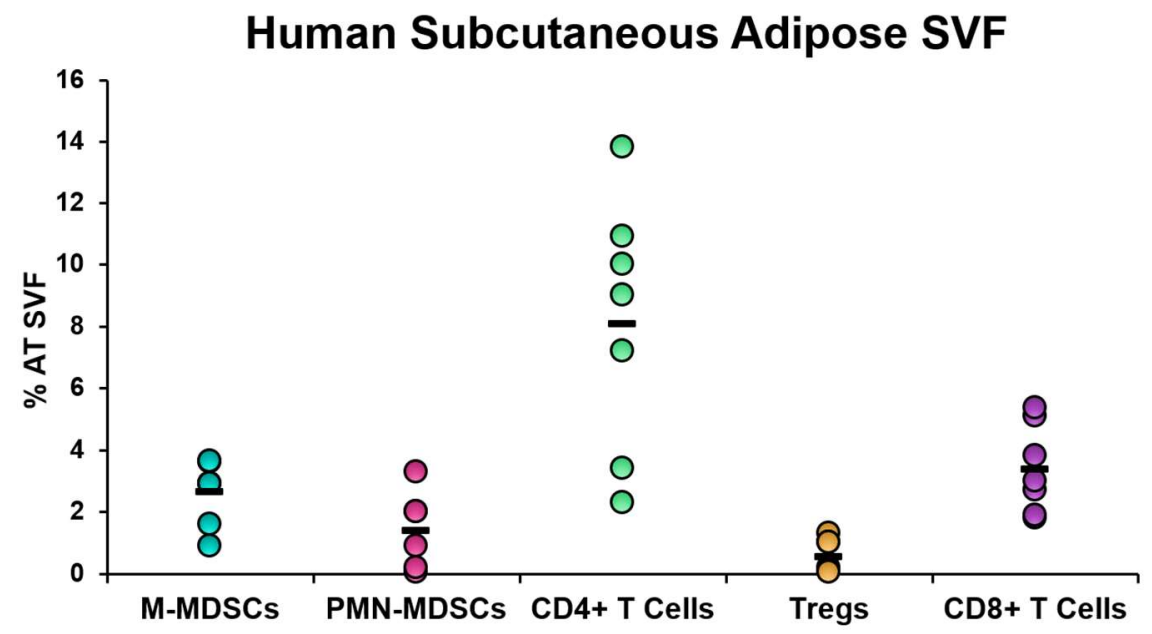

Figure 6.6: Flow cytometric characterization of healthy human adipose tissue. (a) Representative dot plots depicting the MDSC characterization strategy used for human adipose tissue stromal vascular fraction cells (SVF). (b) The frequency of various immune cell populations in human adipose SVF. Each circle represents an individual patient and the horizontal bars represent the mean of the data collected from 6-7 patients. 


\section{FUTURE DIRECTIONS}

This study uncovered an intriguing new layer of the immune cell network in healthy adipose tissue; however, there are a few remaining questions that we were unable to fully investigate.

\section{How do adipose-resident MDSCs prevent $C D 4^{+} T_{E M}$ proliferation under healthy conditions?}

Peroxynitrite-mediated nitrosylation of $\mathrm{T}$ cell receptors (TCR) or MHC-II interferes in the antigen recognition process and is also one of the more indirect mechanisms by which tumor-associated MDSCs modulate T cell responses [157]. These misfolded proteins result from interactions between radical nitrogen species and exposed tyrosine residues on the targeted protein. We hypothesized that nitrosylation of antigen recognition or presentation machinery could explain the delayed onset of aMDSC-mediated immune modulation, since the suppressive effects of this process on $\mathrm{T}$ cell proliferation increases with increased exposure to radical nitrogen species. Unfortunately, we were only able to perform one pilot experiment on the SVF from a healthy mouse, but this did yield promising results. Using flow cytometry, we found that the $\mathrm{CD}^{+}{ }^{+}$and $\mathrm{CD} 8^{+}$adipose tissue $\mathrm{T}$ cells did have more nitrosylated tyrosine residues on their surface than T cells in the spleens of these mice (Figure 6.7).

Although far from conclusive, it is tempting to speculate that this nitrosylation was a direct result of their proximity to aMDSCs. If time had allowed it would definitely have been worthwhile to investigate the nitrosylation state of the CD $301^{+}$ATMs isolated from the SVF of aGr1-treated mice as I suspect that the 
sudden reinstatement of functional antigen presentation machinery may be a large factor in the rapid accumulation of T cells following in vivo MDSC depletion. Additional support for this hypothesis can be found in the literature on $\mathrm{T}$ cell and macrophage dynamics in the early stages of obesity development. These studies have found that the initial accumulation of $\mathrm{CD} 4^{+}$inflammatory $\mathrm{T}$ cells is triggered by an endogenous adipose antigen [210] that is presented to these ATMs in an MHCIl-dependent fashion [142]. If we plug our observations from the MDSC depletion experiments into this working model, it would suggest that aMDSCs in healthy adipose modulate the functionality of antigen presentation machinery on AT macrophages, impeding their ability to inadvertently reactivate AT resident $\mathrm{T}_{\mathrm{EM}}$.

To determine if aMDSC-mediated nitrosylation of antigen presenting and recognizing proteins prevents TEM activation and proliferation, we would expand the preliminary line of investigation to include $\alpha \mathrm{Gr} 1$ depletion experiments for one or four weeks. SVF cells would be stained with T cell and macrophage markers along with the $\mathrm{N}$-tyrosine antibody used in our pilot experiments. Analyzing these samples on an Image Stream would allow us to detect colocalization of nitrosylated tyrosine residues on T cell receptors and MHC proteins. Additional staining could be used to assess whether MDSC-depletion increases surface expression of CD3 $\zeta$ or IL-2R on adipose tissue T cells, as these two proteins are directly involved in antigen-mediated responses and lose functionality as a result of aMDSC nitrosylation. 

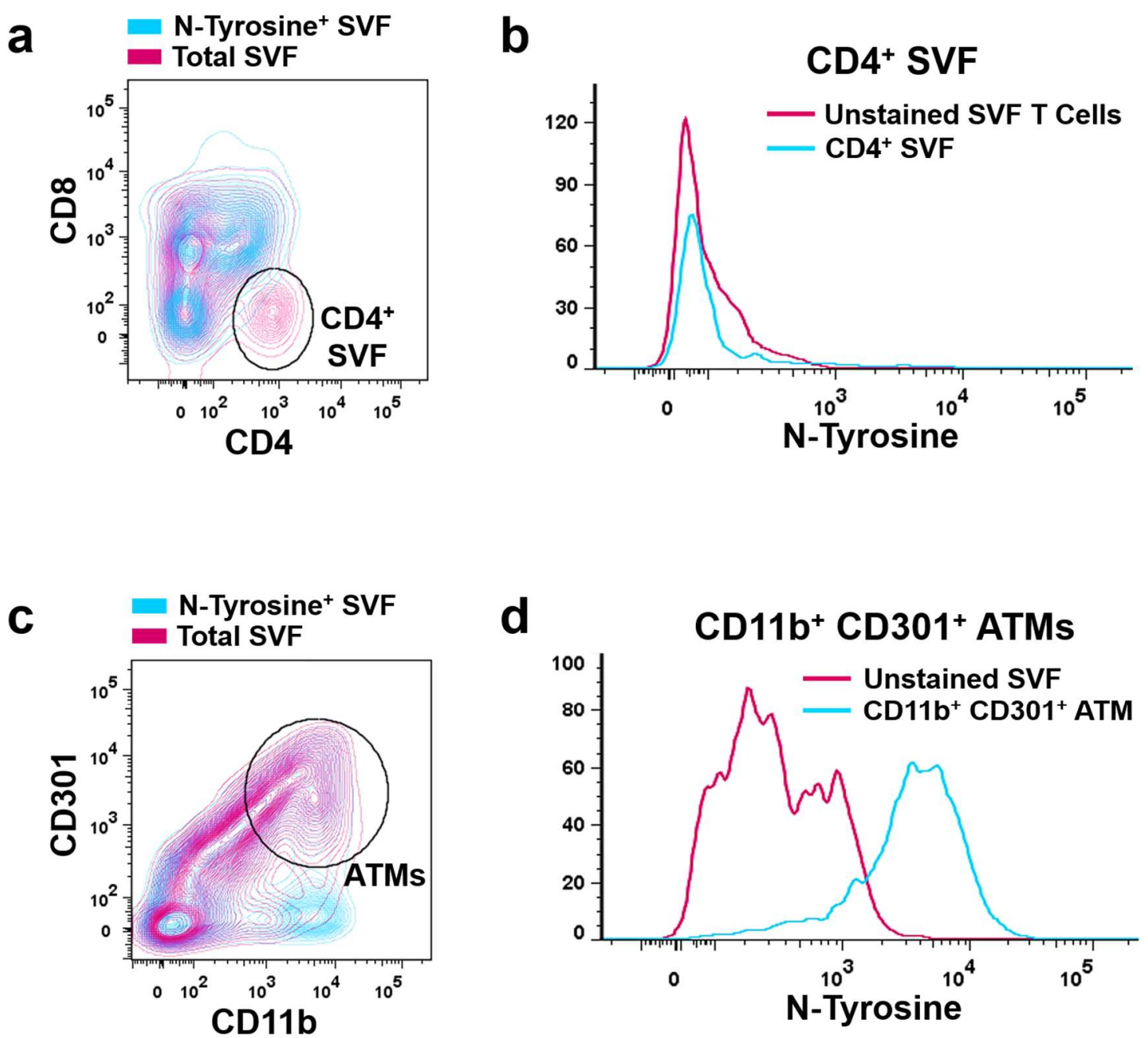

Figure 6.7: Macrophages, but not T cells have nitrosylated surface proteins in healthy adipose SVF. (a) Contour plot comparing CD4 and CD8 expression on nitrosylated tyrosine (N-tyrosine) positive SVF cells (Blue) versus the whole SVF isolate (Pink). (b) Overlaid histograms showing N-tyrosine on the surface of CD4 ${ }^{+}$ SVF T cells (Blue). Unstained SVF T cells (Pink) back-gated based on similar size and granularity (FSC-A vs SSC-A) to $\mathrm{CD}^{+}$SVF. (c) Contour plot comparing CD11b and CD301 expression on N-tyrosine positive SVF cells (Blue) versus the whole SVF isolate (Pink). (d) Overlaid histograms showing $\mathrm{N}$-tyrosine on the surface of $\mathrm{CD}_{0} 1^{+}$adipose tissue macrophages (ATMs) (Blue). Unstained SVF (Pink) back-gated based on similar size and granularity (FSC-A vs SSC-A) to CD11b+CD301+ ATMs. 


\section{What recruits MDSCs to healthy adipose tissue?}

The current thinking on MDSC genesis is that TLR activation recruits circulating monocytes into the tissue or tumor, where they encounter a second TLR agonist, in addition to immunomodulatory signals such as IL-10 and TGF $\beta$. This combination of pro-and anti-inflammatory stimuli "reprograms" the monocyte, causing it to acquire immune suppressive functions [93]. In vivo, conditions such as these typically occur at sites of chronic inflammation where pro-inflammatory Type I responses react to an insult, while tissue remodeling Type II responses attempt to repair and prevent further damage to surrounding tissue. As discussed in the Introduction, a surprisingly similar microenvironmental setting can be found in healthy AT (Figure 6.8). This tissue is constantly remodeling to accommodate for fluctuations in adipocyte size as they store and release lipid [13]. The TNFamediated degradation of the extracellular matrix required for these dynamic structural changes [13] may provide a constitutive source of TLR agonists such as biglycan and decorin [15] which are recognized by TLRs 2 and 4. Once recruited into the tissue, circulating monocytes would encounter the suppressive network of AT-resident immune cells, including aMDSCs, which would provide the secondary immunomodulatory signals, such as IL-10, IL-13, and TGF $\beta$, needed to promote their reprogramming $[29,154]$. This homeostatic oscillation between acute inflammation and its rapid resolution creates an ideal niche for a population of tissue resident MDSCs to thrive. 


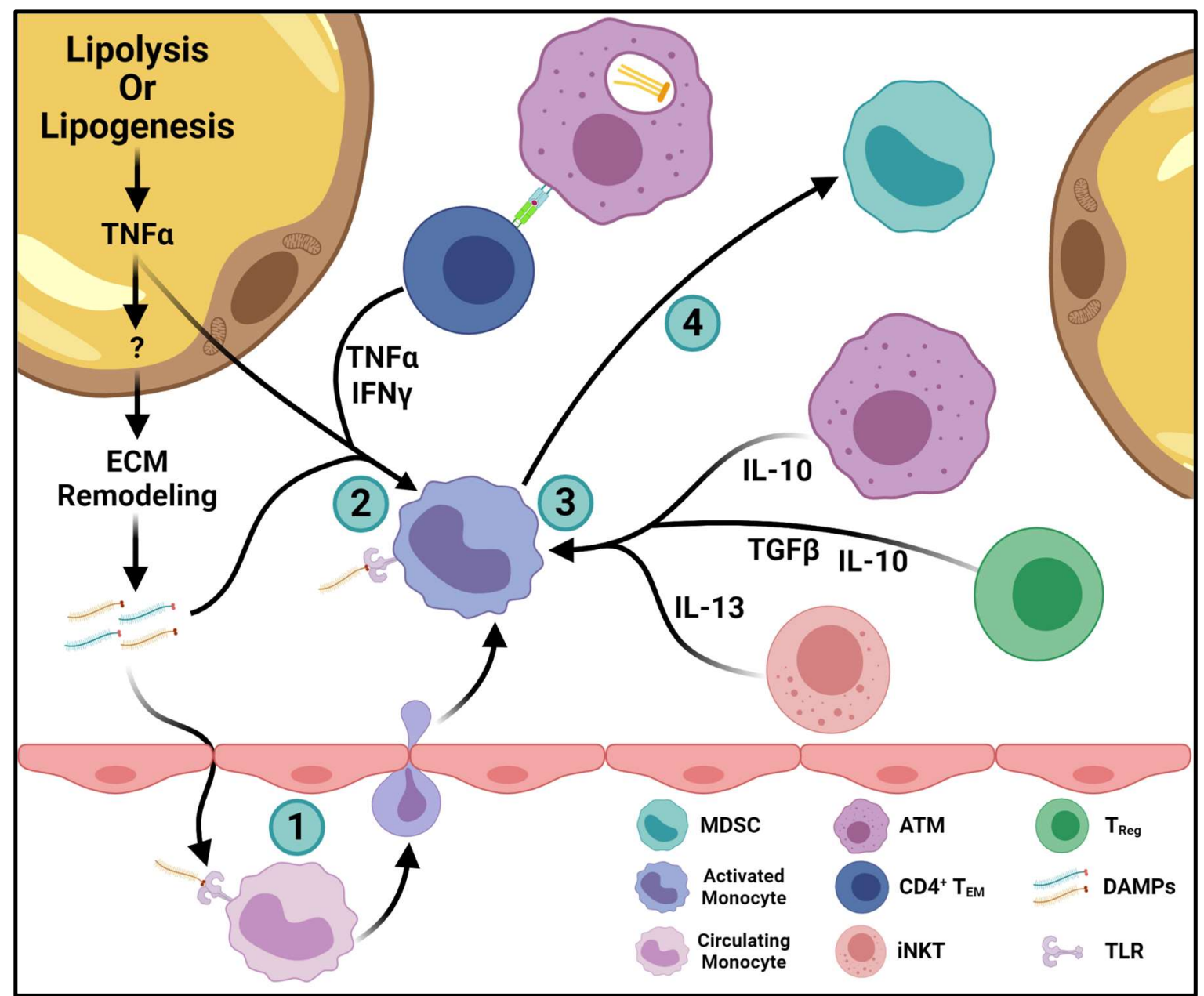

Figure 6.8: Proposed model for MDSC recruitment into healthy adipose tissue.

1) Adipocyte lipolysis or lipogenesis induces the breakdown of the extracellular matrix (ECM). By-products of ECM remodeling can trigger toll-like receptors on circulating monocytes, recruiting them into the tissue.

2) Monocytes encounter pro-inflammatory signals from further TLR stimulation or produced by effector memory T cells (TEM).

3) The abundance of immunomodulatory cytokines found in healthy adipose tissue provide concurrent anti-inflammatory signals to the monocyte.

4) The combination of pro- and anti-inflammatory stimuli reprograms the monocyte to adopt a suppressive MDSC phenotype. 


\section{What is the relationship between MDSCs and metabolic disease?}

For almost a decade the relationship between MDSCs and obesity has been a subject of interest for those investigating alternative therapies for metabolic disease $[97,129,130,211]$. Our work has introduced another layer to this by demonstrating that MDSCs are constitutively present in healthy adipose tissue and not just the inflamed adipose of obesity as described by others. However, if these cells accumulate during obesity and the severity of metabolic symptoms negatively correlates with the number of MDSCs in the tissue [129], then why does adipose become inflamed in the first place and why does chronic high fat diet exacerbate this inflammation even as the MDSC population expands?

One possibility is that the increasingly pro-inflammatory conditions that develop in adipose tissue during high fat diet force their differentiation into "M1"type macrophages. Several lines of evidence from tumor and adipose biology support this consideration. First, the repolarization and forced differentiation of MDSCs into pro-inflammatory macrophages and dendritic cells has been described as a possible strategy in overcoming their potent suppression of antitumor immune responses [212]. Second, further supporting this idea is the accumulation of $\mathrm{CD} 11 \mathrm{c}^{+}$monocyte derived "M1" macrophages in obese adipose tissue [20]. Pro-inflammatory cues recruit circulating monocytes into the adipose which then differentiate into mature antigen presenting cells, capable of inciting inflammatory $\mathrm{T}$ cell responses. Third, these same factors can cause normally suppressive, ATMs to shift towards an "M1" phenotype themselves [29]. This indicates that pro-inflammatory cues within the adipose microenvironment recruit inflammatory cells and actively affect the functional phenotypes of both recruited 
and tissue resident cells. Altogether these studies demonstrate the functional plasticity of MDSCs as well as their sensitivity to shifts in microenvironmental cues. Given MDSC plasticity, it is likely that chronic exposure to the pro-inflammatory microenvironment within obese adipose tissue ultimately leads to their differentiation into "M1" macrophages further propagating obesity-induced inflammation. 


\section{REFERENCES}

1. Peirce, V., S. Carobbio, and A. Vidal-Puig, The different shades of fat. Nature, 2014. 510(7503): p. 76-83.

2. Kane, H. and L. Lynch, Innate Immune Control of Adipose Tissue Homeostasis. Trends Immunol, 2019. 40(9): p. 857-872.

3. Jackson-Jones, L.H., et al., Stromal Cells Covering Omental Fat-Associated Lymphoid Clusters Trigger Formation of Neutrophil Aggregates to Capture Peritoneal Contaminants. Immunity, 2020. 52(4): p. 700-715.e6.

4. Villarroya, F., et al., Toward an Understanding of How Immune Cells Control Brown and Beige Adipobiology. Cell Metab, 2018. 27(5): p. 954-961.

5. Pires de Carvalho, P., et al., Comparison of infrapatellar and subcutaneous adipose tissue stromal vascular fraction and stromal/stem cells in osteoarthritic subjects. J Tissue Eng Regen Med, 2014. 8(10): p. 757-62.

6. Ioan-Facsinay, A. and M. Kloppenburg, An emerging player in knee osteoarthritis: the infrapatellar fat pad. Arthritis Res Ther, 2013. 15(6): p. 225.

7. Ibrahim, M.M., Subcutaneous and visceral adipose tissue: structural and functional differences. Obes Rev, 2010. 11(1): p. 11-8.

8. Caspar-Bauguil, S., et al., Adipose tissues as an ancestral immune organ: sitespecific change in obesity. FEBS Lett, 2005. 579(17): p. 3487-92.

9. Meza-Perez, S. and T.D. Randall, Immunological Functions of the Omentum. Trends in Immunology, 2017. 38(7): p. 526-536.

10. Rangel-Moreno, J., et al., Omental milky spots develop in the absence of lymphoid tissue-inducer cells and support $B$ and $T$ cell responses to peritoneal antigens. Immunity, 2009. 30(5): p. 731-43.

11. Bedford, P.A., et al., Adipose tissue of human omentum is a major source of dendritic cells, which lose MHC Class II and stimulatory function in Crohn's disease. J Leukoc Biol, 2006. 80(3): p. 546-54.

12. Jones, D.D., et al., The omentum is a site of protective IgM production during intracellular bacterial infection. Infect Immun, 2015. 83(5): p. 2139-47.

13. Wernstedt Asterholm, I., et al., Adipocyte Inflammation Is Essential for Healthy Adipose Tissue Expansion and Remodeling. Cell Metabolism, 2014. 20(1): p. 103-118.

14. Martins, L.B., et al., Paradoxical role of tumor necrosis factor on metabolic dysfunction and adipose tissue expansion in mice. Nutrition, 2018. 50: p. 1-7.

15. Frevert, C.W., et al., Danger-Associated Molecular Patterns Derived From the Extracellular Matrix Provide Temporal Control of Innate Immunity. Journal of Histochemistry \& Cytochemistry, 2018. 66(4): p. 213-227.

16. Lu, P., et al., Extracellular matrix degradation and remodeling in development and disease. Cold Spring Harbor perspectives in biology, 2011. 3(12): $p$. 10.1101/cshperspect.a005058 a005058.

17. Ruiz-Ojeda, F.J., et al., Extracellular Matrix Remodeling of Adipose Tissue in Obesity and Metabolic Diseases. Int J Mol Sci, 2019. 20(19). 
18. Svard, J., et al., Absence of the proteoglycan decorin reduces glucose tolerance in overfed male mice. Sci Rep, 2019. 9(1): p. 4614.

19. Asterholm, I.W., et al., Lack of "immunological fitness" during fasting in metabolically challenged animals. J Lipid Res, 2012. 53(7): p. 1254-67.

20. Russo, L. and C.N. Lumeng, Properties and functions of adipose tissue macrophages in obesity. Immunology, 2018. 155(4): p. 407-417.

21. Lumeng, C.N., et al., Phenotypic switching of adipose tissue macrophages with obesity is generated by spatiotemporal differences in macrophage subtypes. Diabetes, 2008. 57(12): p. 3239-46.

22. Hassnain Waqas, S.F., et al., Adipose tissue macrophages develop from bone marrow-independent progenitors in Xenopus laevis and mouse. J Leukoc Biol, 2017. 102(3): p. 845-855.

23. Haase, J., et al., Local proliferation of macrophages in adipose tissue during obesity-induced inflammation. Diabetologia, 2014. 57(3): p. 562-71.

24. Roussel, M., et al., Mass cytometry deep phenotyping of human mononuclear phagocytes and myeloid-derived suppressor cells from human blood and bone marrow. J Leukoc Biol, 2017. 102(2): p. 437-447.

25. Silva, H.M., et al., Vasculature-associated fat macrophages readily adapt to inflammatory and metabolic challenges. The Journal of Experimental Medicine, 2019. 216(4): p. 786-806.

26. $\mathrm{Xu}, \mathrm{X}$., et al., Obesity activates a program of lysosomal-dependent lipid metabolism in adipose tissue macrophages independently of classic activation. Cell Metab, 2013. 18(6): p. 816-30.

27. Caspar-Bauguil, S., et al., Fatty acids from fat cell lipolysis do not activate an inflammatory response but are stored as triacylglycerols in adipose tissue macrophages. Diabetologia, 2015. 58(11): p. 2627-36.

28. Kosteli, A., et al., Weight loss and lipolysis promote a dynamic immune response in murine adipose tissue. J Clin Invest, 2010. 120(10): p. 3466-79.

29. Lumeng, C.N., J.L. Bodzin, and A.R. Saltiel, Obesity induces a phenotypic switch in adipose tissue macrophage polarization. J Clin Invest, 2007. 117(1): p. 175-84

30. Molofsky, A.B., et al., Innate lymphoid type 2 cells sustain visceral adipose tissue eosinophils and alternatively activated macrophages. J Exp Med, 2013. 210(3): p. 535-49.

31. Bénézech, C. and L.H. Jackson-Jones, ILC2 Orchestration of Local Immune Function in Adipose Tissue. Frontiers in Immunology, 2019. 10(10): p. 171.

32. Hashiguchi, M., et al., IL-33 activates eosinophils of visceral adipose tissue both directly and via innate lymphoid cells. Eur J Immunol, 2015. 45(3): p. 876-85.

33. Molofsky, A.B., A.K. Savage, and R.M. Locksley, Interleukin-33 in Tissue Homeostasis, Injury, and Inflammation. Immunity, 2015. 42(6): p. 1005-19.

34. Cautivo, K.M. and A.B. Molofsky, Regulation of metabolic health and adipose tissue function by group 2 innate lymphoid cells. European journal of immunology, 2016. 46(6): p. 1315-1325.

35. Wu, D., et al., Eosinophils sustain adipose alternatively activated macrophages associated with glucose homeostasis. Science, 2011. 332(6026): p. 243-7.

36. Park, M.J., et al., Adipose tissue-derived mesenchymal stem cells induce expansion of Interleukin-10 producing regulatory $B$ cells and ameliorate autoimmunity in a murine model of systemic lupus erythematosus. Cell Transplant, 2014. 
37. Nishimura, S., et al., Adipose Natural Regulatory B Cells Negatively Control Adipose Tissue Inflammation. Cell Metab, 2013.

38. Yang, $\mathrm{H}$., et al., Obesity increases the production of proinflammatory mediators from adipose tissue T cells and compromises TCR repertoire diversity: implications for systemic inflammation and insulin resistance. J Immunol, 2010. 185(3): p. 1836-45.

39. Han, S.J., et al., White Adipose Tissue Is a Reservoir for Memory T Cells and Promotes Protective Memory Responses to Infection. Immunity, 2017. 47(6): p. 1154-1168.e6.

40. Kawabe, T., et al., Memory-phenotype CD4(+) T cells spontaneously generated under steady-state conditions exert innate TH1-like effector function. Sci Immunol, 2017. 2(12).

41. Chun, T.W., S. Moir, and A.S. Fauci, HIV reservoirs as obstacles and opportunities for an HIV cure. Nat Immunol, 2015. 16(6): p. 584-9.

42. Couturier, J., et al., Human adipose tissue as a reservoir for memory CD4+ $T$ cells and HIV. AIDS, 2015. 29(6): p. 667-74.

43. Teixeira, L., et al., Immune response in the adipose tissue of lean mice infected with the protozoan parasite Neospora caninum. Immunology, 2015. 145(2): p. 242-57.

44. Bouzid, F., et al., Mycobacterium canettii Infection of Adipose Tissues. Front Cell Infect Microbiol, 2017. 7: p. 189.

45. Damouche, A., et al., High proportion of PD-1-expressing CD4(+) T cells in adipose tissue constitutes an immunomodulatory microenvironment that may support HIV persistence. Eur J Immunol, 2017. 47(12): p. 2113-2123.

46. Tanowitz, H.B., et al., Adipose Tissue: A Safe Haven for Parasites? Trends Parasitol, 2017. 33(4): p. 276-284.

47. Couturier, J. and D.E. Lewis, HIV Persistence in Adipose Tissue Reservoirs. Curr HIVIAIDS Rep, 2018. 15(1): p. 60-71.

48. Wanjalla, C.N., W.J. McDonnell, and J.R. Koethe, Adipose Tissue T Cells in HIV/SIV Infection. Front Immunol, 2018. 9: p. 2730.

49. Bapat, S.P., et al., Depletion of fat-resident $T_{\text {reg }}$ cells prevents age-associated insulin resistance. Nature, 2015. 528(7580): p. 137-41.

50. Feuerer, M., et al., Lean, but not obese, fat is enriched for a unique population of regulatory $T$ cells that affect metabolic parameters. Nat Med, 2009. 15(8): p. 9309.

51. Han, S., et al., Adipose-Derived Stromal Vascular Fraction Cells: Update on Clinical Utility and Efficacy. Crit Rev Eukaryot Gene Expr, 2015. 25(2): p. 145-52.

52. Qin, Y., et al., The adipose-derived lineage-negative cells are enriched mesenchymal stem cells and promote limb ischemia recovery in mice. Stem Cells Dev, 2014. 23(4): p. 363-71.

53. Dong, Z., et al., The survival condition and immunoregulatory function of adipose stromal vascular fraction (SVF) in the early stage of nonvascularized adipose transplantation. PLoS One, 2013. 8(11): p. e80364.

54. Bowles, A.C., et al., Adipose Stromal Vascular Fraction-Mediated Improvements at Late-Stage Disease in a Murine Model of Multiple Sclerosis. Stem Cells, 2017. 35(2): p. 532-544.

55. Semon, J.A., et al., Administration of murine stromal vascular fraction ameliorates chronic experimental autoimmune encephalomyelitis. Stem Cells Transl Med, 2013. 2(10): p. 789-96. 
56. Rigotti, G., et al., Expanded Stem Cells, Stromal-Vascular Fraction, and PlateletRich Plasma Enriched Fat: Comparing Results of Different Facial Rejuvenation Approaches in a Clinical Trial. Aesthet Surg J, 2016. 36(3): p. 261-70.

57. Zhou, L., et al., Comparison of human adipose stromal vascular fraction and adipose-derived mesenchymal stem cells for the attenuation of acute renal ischemia/reperfusion injury. Sci Rep, 2017. 7: p. 44058.

58. Carstens, M.H., et al., Non-reconstructable peripheral vascular disease of the lower extremity in ten patients treated with adipose-derived stromal vascular fraction cells. Stem Cell Res, 2017. 18: p. 14-21.

59. Wu, L., et al., Chondrocytes Cocultured with Stromal Vascular Fraction of Adipose Tissue Present More Intense Chondrogenic Characteristics Than with Adipose Stem Cells. Tissue Eng Part A, 2016. 22(3-4): p. 336-48.

60. Zuk, P.A., et al., Multilineage cells from human adipose tissue: implications for cell-based therapies. Tissue Eng, 2001. 7(2): p. 211-28.

61. Plock, J.A., et al., Perspectives on the Use of Mesenchymal Stem Cells in Vascularized Composite Allotransplantation. Frontiers in Immunology, 2013. 4: p. 175.

62. Bajek, A., et al., Adipose-Derived Stem Cells as a Tool in Cell-Based Therapies. Arch Immunol Ther Exp (Warsz), 2016. 64(6): p. 443-454.

63. Melief, S.M., et al., Adipose tissue-derived multipotent stromal cells have a higher immunomodulatory capacity than their bone marrow-derived counterparts. Stem Cells Transl Med, 2013. 2(6): p. 455-63.

64. Plock, J.A., et al., The Influence of Timing and Frequency of Adipose-Derived Mesenchymal Stem Cell Therapy on Immunomodulation Outcomes After Vascularized Composite Allotransplantation. Transplantation, 2017. 101(1): p. e1-e11.

65. Schweizer, R., V.S. Gorantla, and J.A. Plock, Premise and promise of mesenchymal stem cell-based therapies in clinical vascularized composite allotransplantation. Curr Opin Organ Transplant, 2015. 20(6): p. 608-14.

66. Tsuji, W., et al., Effects of immunosuppressive drugs on viability and susceptibility of adipose- and bone marrow-derived mesenchymal stem cells. Front Immunol, 2015. 6: p. 131.

67. Valencia, J., et al., Comparative analysis of the immunomodulatory capacities of human bone marrow- and adipose tissue-derived mesenchymal stromal cells from the same donor. Cytotherapy, 2016. 18(10): p. 1297-311.

68. Zamora, R., et al., Differential inflammatory networks distinguish responses to bone marrow-derived vs. adipose-derived mesenchymal stem cell therapies in vascularized composite allotransplantation. J Trauma Acute Care Surg, 2017.

69. Wan, C.D., et al., Immunomodulatory effects of mesenchymal stem cells derived from adipose tissues in a rat orthotopic liver transplantation model. Hepatobiliary Pancreat Dis Int, 2008. 7(1): p. 29-33.

70. Liu, T., et al., Immunomodulatory effects of OX40Ig gene-modified adipose tissue-derived mesenchymal stem cells on rat kidney transplantation. Int J Mol Med, 2017. 39(1): p. 144-152.

71. Jeong, S.H., Y.H. Ji, and E.S. Yoon, Immunosuppressive Activity of Adipose Tissue-Derived Mesenchymal Stem Cells in a Rat Model of Hind Limb Allotransplantation. Transplantation Proceedings, 2014. 46(5): p. 1606-1614.

72. Luz-Crawford, P., C. Jorgensen, and F. Djouad, Mesenchymal Stem Cells Direct the Immunological Fate of Macrophages. Results Probl Cell Differ, 2017. 62: p. $61-72$. 
73. Lopez-Santalla, M., et al., Adipose-derived mesenchymal stromal cells modulate experimental autoimmune arthritis by inducing an early regulatory innate cell signature. Immun Inflamm Dis, 2016. 4(2): p. 213-224.

74. Ko, J.H., et al., Mesenchymal stem/stromal cells precondition lung monocytes/macrophages to produce tolerance against allo- and autoimmunity in the eye. Proc Natl Acad Sci U S A, 2016. 113(1): p. 158-63.

75. Xie, J., et al., Human Adipose-Derived Stem Cells Suppress Elastase-Induced Murine Abdominal Aortic Inflammation and Aneurysm Expansion Through Paracrine Factors. Cell Transplant, 2017. 26(2): p. 173-189.

76. Ivanova-Todorova, E., et al., Adipose tissue-derived mesenchymal stem cells are more potent suppressors of dendritic cells differentiation compared to bone marrow-derived mesenchymal stem cells. Immunol Lett, 2009. 126(1-2): p. 3742.

77. Feng, Z., et al., Fresh and cryopreserved, uncultured adipose tissue-derived stem and regenerative cells ameliorate ischemia-reperfusion-induced acute kidney injury. Nephrol Dial Transplant, 2010. 25(12): p. 3874-84.

78. You, D., et al., Comparative study of autologous stromal vascular fraction and adipose-derived stem cells for erectile function recovery in a rat model of cavernous nerve injury. Stem Cells Transl Med, 2015. 4(4): p. 351-8.

79. Klar, A.S., et al., Characterization of vasculogenic potential of human adiposederived endothelial cells in a three-dimensional vascularized skin substitute. Pediatr Surg Int, 2016. 32(1): p. 17-27.

80. Semon, J.A., et al., Comparison of human adult stem cells from adipose tissue and bone marrow in the treatment of experimental autoimmune encephalomyelitis. Stem Cell Res Ther, 2014. 5(1): p. 2.

81. Behfar, M., S. Javanmardi, and F. Sarrafzadeh-Rezaei, Comparative study on functional effects of allotransplantation of bone marrow stromal cells and adipose derived stromal vascular fraction on tendon repair: a biomechanical study in rabbits. Cell J, 2014. 16(3): p. 263-70.

82. Daumas, A., et al., Long-term follow-up after autologous adipose-derived stromal vascular fraction injection into fingers in systemic sclerosis patients. Curr Res Transl Med, 2017. 65(1): p. 40-43.

83. Iddins, C.J., et al., Case Report: Industrial X-Ray Injury Treated With NonCultured Autologous Adipose-Derived Stromal Vascular Fraction (SVF). Health Phys, 2016. 111(2): p. 112-6.

84. Jones, C.M., et al. Administration of Recipient Adipose Stromal Vascular Fraction (RA-SVF) cells in clinical hand transplant recipients. in 13th Congress of International Society of Vascularized Composite Allotransplantation October 2627, 2017. Salzburg, Austria.

85. Morris, M.E., et al., Systemically delivered adipose stromal vascular fraction cells disseminate to peripheral artery walls and reduce vasomotor tone through a $C D 11 b^{+}$cell-dependent mechanism. Stem Cells Transl Med, 2015. 4(4): p. 36980.

86. Talukdar, S., et al., Neutrophils mediate insulin resistance in mice fed a high-fat diet through secreted elastase. Nat Med, 2012. 18(9): p. 1407-12.

87. Ma, H. and C.Q. Xia, Phenotypic and Functional Diversities of Myeloid-Derived Suppressor Cells in Autoimmune Diseases. Mediators Inflamm, 2018. 2018: p. 4316584. 
88. Wegner, A., J. Verhagen, and D.C. Wraith, Myeloid-derived suppressor cells mediate tolerance induction in autoimmune disease. Immunology, 2017. 151(1): p. 26-42.

89. Crook, K.R., et al., Myeloid-derived suppressor cells regulate T cell and B cell responses during autoimmune disease. J Leukoc Biol, 2015. 97(3): p. 573-82.

90. Ostrand-Rosenberg, S., et al., Frontline Science: Myeloid-derived suppressor cells (MDSCs) facilitate maternal-fetal tolerance in mice. J Leukoc Biol, 2017. 101(5): p. 1091-1101.

91. Lv, M., et al., Monocytic and promyelocytic myeloid-derived suppressor cells may contribute to G-CSF-induced immune tolerance in haplo-identical allogeneic hematopoietic stem cell transplantation. Am J Hematol, 2015. 90(1): p. E9-E16.

92. Hongo, D., et al., Requirement for interactions of natural killer T cells and myeloid-derived suppressor cells for transplantation tolerance. Am J Transplant, 2014. 14(11): p. 2467-77.

93. Millrud, C.R., C. Bergenfelz, and K. Leandersson, On the origin of myeloidderived suppressor cells. Oncotarget, 2016. 8: p. 3649-3665.

94. Yang, $\mathrm{H}$., et al., Myeloid-derived suppressor cells in immunity and autoimmunity. Expert Rev Clin Immunol, 2015. 11(8): p. 911-9.

95. Mastorodemos, V., M. Ioannou, and P. Verginis, Cell-based modulation of autoimmune responses in multiple sclerosis and experimental autoimmmune encephalomyelitis: therapeutic implications. Neuroimmunomodulation, 2015. 22(3): p. 181-95.

96. Saiwai, H., et al., Ly6C $C^{+}$Ly6G- Myeloid-derived suppressor cells play a critical role in the resolution of acute inflammation and the subsequent tissue repair process after spinal cord injury. J Neurochem, 2013. 125(1): p. 74-88.

97. Pawelec, G., C.P. Verschoor, and S. Ostrand-Rosenberg, Myeloid-Derived Suppressor Cells: Not Only in Tumor Immunity. Front Immunol, 2019. 10: p. 1099.

98. Marvel, D. and D.I. Gabrilovich, Myeloid-derived suppressor cells in the tumor microenvironment: expect the unexpected. J Clin Invest, 2015. 125(9): p. 335664.

99. Gabrilovich, D.I. and S. Nagaraj, Myeloid-derived-suppressor cells as regulators of the immune system. Nat Rev Immunol, 2009. 9(3): p. 162-74.

100. Bronte, V., et al., Recommendations for myeloid-derived suppressor cell nomenclature and characterization standards. Nature communications, 2016. 7: p. $12150-12150$.

101. Tcyganov, E., et al., Plasticity of myeloid-derived suppressor cells in cancer. Curr Opin Immunol, 2018. 51: p. 76-82.

102. Kumar, V., et al., The Nature of Myeloid-Derived Suppressor Cells in the Tumor Microenvironment. Trends in Immunology, 2016. 37(3): p. 208-220.

103. Youn, J.I. and D.I. Gabrilovich, The biology of myeloid-derived suppressor cells: The blessing and the curse of morphological and functional heterogeneity. Eur $\mathrm{J}$ Immunol, 2010. 40(11): p. 2969-75.

104. Rodriguez, P.C., et al., Arginase I production in the tumor microenvironment by mature myeloid cells inhibits $T$-cell receptor expression and antigen-specific $T$ cell responses. Cancer Res, 2004. 64(16): p. 5839-49.

105. Cimen Bozkus, C., et al., Expression of Cationic Amino Acid Transporter 2 Is Required for Myeloid-Derived Suppressor Cell-Mediated Control of T Cell Immunity. Journal of immunology (Baltimore, Md. : 1950), 2015. 195(11): p. 5237-5250. 
106. Srivastava, M.K., et al., Myeloid-derived suppressor cells inhibit T-cell activation by depleting cystine and cysteine. Cancer Res, 2010. 70(1): p. 68-77.

107. Gabrilovich, D.I., S. Ostrand-Rosenberg, and V. Bronte, Coordinated regulation of myeloid cells by tumours. Nat Rev Immunol, 2012. 12(4): p. 253-68.

108. Schmielau, J. and O.J. Finn, Activated granulocytes and granulocyte-derived hydrogen peroxide are the underlying mechanism of suppression of $t$-cell function in advanced cancer patients. Cancer Res, 2001. 61(12): p. 4756-60.

109. Mazzoni, A., et al., Myeloid suppressor lines inhibit $T$ cell responses by an NOdependent mechanism. J Immunol, 2002. 168(2): p. 689-95.

110. Molon, B., et al., Chemokine nitration prevents intratumoral infiltration of antigenspecific T cells. J Exp Med, 2011. 208(10): p. 1949-62.

111. Hanson, E.M., et al., Myeloid-derived suppressor cells down-regulate L-selectin expression on CD4+ and CD8+ T cells. J Immunol, 2009. 183(2): p. 937-44.

112. Sakuishi, K., et al., Emerging Tim-3 functions in antimicrobial and tumor immunity. Trends Immunol, 2011. 32(8): p. 345-9.

113. Li, H., et al., Cancer-expanded myeloid-derived suppressor cells induce anergy of NK cells through membrane-bound TGF-beta 1. J Immunol, 2009. 182(1): p. 240-9.

114. Beury, D.W., et al., Cross-talk among myeloid-derived suppressor cells, macrophages, and tumor cells impacts the inflammatory milieu of solid tumors. Journal of Leukocyte Biology, 2014. 96(6): p. 1109-1118.

115. Ostrand-Rosenberg, S., et al., Cross-talk between myeloid-derived suppressor cells (MDSC), macrophages, and dendritic cells enhances tumor-induced immune suppression. Semin Cancer Biol, 2012. 22(4): p. 275-81.

116. Huang, B., et al., Gr-1+CD115+ immature myeloid suppressor cells mediate the development of tumor-induced T regulatory cells and T-cell anergy in tumorbearing host. Cancer Res, 2006. 66(2): p. 1123-31.

117. Pan, P.Y., et al., Immune stimulatory receptor CD40 is required for T-cell suppression and $T$ regulatory cell activation mediated by myeloid-derived suppressor cells in cancer. Cancer Res, 2010. 70(1): p. 99-108.

118. Serafini, P., et al., Myeloid-derived suppressor cells promote cross-tolerance in $B$-cell lymphoma by expanding regulatory $T$ cells. Cancer Res, 2008. 68(13): p. 5439-49.

119. Panopoulos, A.D. and S.S. Watowich, Granulocyte colony-stimulating factor: molecular mechanisms of action during steady state and 'emergency' hematopoiesis. Cytokine, 2008. 42(3): p. 277-88.

120. Gabrilovich, D., et al., Vascular endothelial growth factor inhibits the development of dendritic cells and dramatically affects the differentiation of multiple hematopoietic lineages in vivo. Blood, 1998. 92(11): p. 4150-66.

121. Cheng, P., et al., Inhibition of dendritic cell differentiation and accumulation of myeloid-derived suppressor cells in cancer is regulated by S100A9 protein. J Exp Med, 2008. 205(10): p. 2235-49.

122. Obermajer, N., et al., Positive feedback between PGE2 and COX2 redirects the differentiation of human dendritic cells toward stable myeloid-derived suppressor cells. Blood, 2011. 118(20): p. 5498-505.

123. Mao, Y., et al., Melanoma-educated CD14+ cells acquire a myeloid-derived suppressor cell phenotype through COX-2-dependent mechanisms. Cancer Res, 2013. 73(13): p. 3877-87.

124. Yu, H., D. Pardoll, and R. Jove, STATs in cancer inflammation and immunity: a leading role for STAT3. Nat Rev Cancer, 2009. 9(11): p. 798-809. 
125. Brudecki, L., et al., Myeloid-derived suppressor cells evolve during sepsis and can enhance or attenuate the systemic inflammatory response. Infect Immun, 2012. 80(6): p. 2026-34.

126. Youn, J.I., et al., Characterization of the nature of granulocytic myeloid-derived suppressor cells in tumor-bearing mice. J Leukoc Biol, 2012. 91(1): p. 167-81.

127. Park, M.-J., et al., Interleukin-10 produced by myeloid-derived suppressor cells is critical for the induction of Tregs and attenuation of rheumatoid inflammation in mice. Scientific Reports, 2018. 8(1): p. 3753.

128. He, Y.-M., et al., Transitory presence of myeloid-derived suppressor cells in neonates is critical for control of inflammation. Nature medicine, 2018. 24(2): p. 224-231.

129. Xia, S., et al., Gr-1+ $C D 11 b^{+}$myeloid-derived suppressor cells suppress inflammation and promote insulin sensitivity in obesity. J Biol Chem, 2011. 286(26): p. 23591-9.

130. Clements, V.K., et al., Frontline Science: High fat diet and leptin promote tumor progression by inducing myeloid-derived suppressor cells. Journal of Leukocyte Biology, 2018. 103(3): p. 395-407.

131. Cox, N. and F. Geissmann, Macrophage ontogeny in the control of adipose tissue biology. Curr Opin Immunol, 2019. 62: p. 1-8.

132. Chung, K.J., et al., Innate immune cells in the adipose tissue. Rev Endocr Metab Disord, 2018. 19(4): p. 283-292.

133. Bunt, S.K., et al., Inflammation enhances myeloid-derived suppressor cell crosstalk by signaling through Toll-like receptor 4. J Leukoc Biol, 2009. 85(6): p. 9961004.

134. Tomic, S., et al., Prostaglanin-E2 Potentiates the Suppressive Functions of Human Mononuclear Myeloid-Derived Suppressor Cells and Increases Their Capacity to Expand IL-10-Producing Regulatory T Cell Subsets. Front Immunol, 2019. 10: p. 475.

135. Krakow, S., et al., Re-expression of CD14 in Response to a Combined IL-10/TLR Stimulus Defines Monocyte-Derived Cells With an Immunoregulatory Phenotype. Front Immunol, 2019. 10: p. 1484.

136. Gras Navarro, A., A.T. Bjorklund, and M. Chekenya, Therapeutic potential and challenges of natural killer cells in treatment of solid tumors. Front Immunol, 2015. 6: p. 202.

137. McEwen-Smith, R.M., M. Salio, and V. Cerundolo, The Regulatory Role of Invariant NKT Cells in Tumor Immunity. Cancer Immunol Res, 2015. 3(5): p. 425435.

138. Hinshaw, D.C. and L.A. Shevde, The Tumor Microenvironment Innately Modulates Cancer Progression. Cancer Research, 2019. 79(18): p. 4557-4566.

139. Brooks, D.L. and T.N. Seagroves, Fluorescence-Activated Cell Sorting of Murine Mammary Cancer Stem-Like Cell Subpopulations with HIF Activity. Methods Mol Biol, 2018. 1742: p. 247-263.

140. Llufrio, E.M., et al., Sorting cells alters their redox state and cellular metabolome. Redox Biology, 2018. 16: p. 381-387.

141. Morris, D.L., et al., CD40 promotes MHC class II expression on adipose tissue macrophages and regulates adipose tissue $C D 4^{+} T$ cells with obesity. J Leukoc Biol, 2016. 99(6): p. 1107-19.

142. Cho, Kae W., et al., An MHC II-Dependent Activation Loop between Adipose Tissue Macrophages and CD4+ T Cells Controls Obesity-Induced Inflammation. Cell Reports, 2014. 9(2): p. 605-617. 
143. Urasaki, Y., et al., Imaging immune and metabolic cells of visceral adipose tissues with multimodal nonlinear optical microscopy. PloS one, 2012. 7(6): p. e38418-e38418.

144. Shen, Y., et al., The Characteristics of Intrinsic Fluorescence of Type I Collagen Influenced by Collagenase I. Applied Sciences, 2018. 8(10): p. 1947.

145. Fujimoto, D., K.-y. Akiba, and N. Nakamura, Isolation and characterization of a fluorescent material in bovine achilles tendon collagen. Biochemical and Biophysical Research Communications, 1977. 76(4): p. 1124-1129.

146. Lodish, H., et al., Section 22.3 Collagen: The Fibrous Proteins of the Matrix, in Molecular Cell Biology, W.H. Freeman, Editor. 2000, Freeman \& Co.: New York, NY.

147. Aikio, M., et al., Specific collagen XVIII isoforms promote adipose tissue accrual via mechanisms determining adipocyte number and affect fat deposition. Proceedings of the National Academy of Sciences, 2014. 111(30): p. E3043E3052.

148. Monici, M., Cell and tissue autofluorescence research and diagnostic application. Biotechnology annual review, 2005. 11: p. 227-56.

149. Hessle, H. and E. Engvall, Type VI Collagen: Studies on its localization, structure, and biosynthetic form with monoclonal antibodies. The Journal of Biological Chemistry, 1984. 259(March 25): p. 3955-3961.

150. Strieder-Barboza, C., et al., Advanced glycation end-products regulate extracellular matrix-adipocyte metabolic crosstalk in diabetes. Scientific reports, 2019. 9(1): p. 19748-19748.

151. Kelm, N.Q., et al., Adipose-derived cells improve left ventricular diastolic function and increase microvascular perfusion in advanced age. PLoS One, 2018. 13(8): p. e0202934.

152. Leblanc, A.J., et al., Adipose-derived cell construct stabilizes heart function and increases microvascular perfusion in an established infarct. Stem Cells Transl Med, 2013. 2(11): p. 896-905.

153. Morris, D.L., et al., $C X_{3} C R 1$ Deficiency Does Not Influence Trafficking of Adipose Tissue Macrophages in Mice With Diet-Induced Obesity. Obesity, 2012. 20(6): p. 1189-1199.

154. Grant, R.W. and V.D. Dixit, Adipose tissue as an immunological organ. Obesity (Silver Spring), 2015. 23(3): p. 512-8.

155. Elgazar-Carmon, V., et al., Neutrophils transiently infiltrate intra-abdominal fat early in the course of high-fat feeding. J Lipid Res, 2008. 49(9): p. 1894-903.

156. Abbas, A.K., et al., Cells and Tissues of the Immune System, in Cellular and Molecular Immunology. 2018, Elsevier. p. 13-37.

157. Nagaraj, S., et al., Mechanism of T cell tolerance induced by myeloid-derived suppressor cells. J Immunol, 2010. 184(6): p. 3106-16.

158. Lindau, D., et al., The immunosuppressive tumour network: myeloid-derived suppressor cells, regulatory T cells and natural killer T cells. Immunology, 2013. 138(2): p. 105-15.

159. Serafini, P., Myeloid derived suppressor cells in physiological and pathological conditions: the good, the bad, and the ugly. Immunol Res, 2013. 57(1-3): p. 17284.

160. Daley, J.M., et al., Use of Ly6G-specific monoclonal antibody to deplete neutrophils in mice. Journal of Leukocyte Biology, 2008. 83(1): p. 64-70. 
161. Pruijt, J.F.M., et al., Neutrophils are indispensable for hematopoietic stem cell mobilization induced by interleukin-8 in mice. Proceedings of the National Academy of Sciences, 2002. 99(9): p. 6228-6233.

162. Garcia, M.R., et al., Monocytic suppressive cells mediate cardiovascular transplantation tolerance in mice. The Journal of Clinical Investigation, 2010. 120(7): p. 2486-2496.

163. Wojtasiak, M., et al., Depletion of $\mathrm{Gr}-1+$, but not Ly6G+, immune cells exacerbates virus replication and disease in an intranasal model of herpes simplex virus type 1 infection. Journal of General Virology, 2010. 91(9): p. 21582166.

164. Johnson, H.L., et al., CD8 T cell-initiated blood-brain barrier disruption is independent of neutrophil support. Journal of immunology (Baltimore, Md. : 1950), 2012. 189(4): p. 1937-1945.

165. Binsfeld, M., et al., Granulocytic myeloid-derived suppressor cells promote angiogenesis in the context of multiple myeloma. Oncotarget, 2016. 7(25): p. 37931-37943.

166. Nakano, H., M. Yanagita, and M.D. Gunn, CD11C(+)B220(+)Gr-1(+) cells in mouse lymph nodes and spleen display characteristics of plasmacytoid dendritic cells. J Exp Med, 2001. 194(8): p. 1171-8.

167. Matsuzaki, J., et al., Successful elimination of memory-type CD8+ $T$ cell subsets by the administration of anti-Gr-1 monoclonal antibody in vivo. Cellular immunology, 2003. 224(2): p. 98-105.

168. Ma, C. and T.F. Greten, Editorial: "Invisible" MDSC in tumor-bearing individuals after antibody depletion: fact or fiction? Journal of Leukocyte Biology, 2016. 99(6): p. 794.

169. Ma, C., et al., Anti-Gr-1 antibody depletion fails to eliminate hepatic myeloidderived suppressor cells in tumor-bearing mice. J Leukoc Biol, 2012. 92(6): p. 1199-206.

170. Moses, K., et al., Survival of residual neutrophils and accelerated myelopoiesis limit the efficacy of antibody-mediated depletion of $L y-6 \mathrm{G}^{+}$cells in tumor-bearing mice. Journal of Leukocyte Biology, 2016. 99(June 2016): p. 811-823.

171. Huh, J.Y., et al., Crosstalk between adipocytes and immune cells in adipose tissue inflammation and metabolic dysregulation in obesity. Mol Cells, 2014. 37(5): p. 365-71.

172. Lee, B.C. and J. Lee, Cellular and molecular players in adipose tissue inflammation in the development of obesity-induced insulin resistance. Biochim Biophys Acta, 2014. 1842(3): p. 446-62.

173. Lumeng, C.N., et al., Aging is associated with an increase in T cells and inflammatory macrophages in visceral adipose tissue. J Immunol, 2011. 187(12): p. 6208-16.

174. Weisberg, S.P., et al., Obesity is associated with macrophage accumulation in adipose tissue. J Clin Invest, 2003. 112(12): p. 1796-808.

175. Scott, K.A., S.J. Melhorn, and R.R. Sakai, Effects of Chronic Social Stress on Obesity. Current obesity reports, 2012. 1(1): p. 16-25.

176. Bartolomucci, A., et al., Metabolic consequences and vulnerability to diet-induced obesity in male mice under chronic social stress. PLoS One, 2009. 4(1): p. e4331.

177. Srivastava, M.K., et al., Myeloid suppressor cell depletion augments antitumor activity in lung cancer. PLoS One, 2012. 7(7): p. e40677. 
178. Tavazoie, M.F., et al., LXR/ApoE Activation Restricts Innate Immune Suppression in Cancer. Cell, 2018. 172(4): p. 825-840.e18.

179. Cibrian, D. and F. Sanchez-Madrid, CD69: from activation marker to metabolic gatekeeper. Eur J Immunol, 2017. 47(6): p. 946-953.

180. Cipolletta, D., et al., PPAR is a major driver of the accumulation and phenotype of adipose tissue $T_{\text {reg }}$ cells. Nature, 2012. 486(7404): p. 549-53.

181. Bassaganya-Riera, J., et al., PPAR $\gamma$ is highly expressed in F4/80hi adipose tissue macrophages and dampens adipose-tissue inflammation. Cell Immunol, 2009. 258(2): p. 138-46.

182. Vasanthakumar, A., et al., The transcriptional regulators IRF4, BATF and IL-33 orchestrate development and maintenance of adipose tissue-resident regulatory $T$ cells. Nat Immunol, 2015. 16(3): p. 276-85.

183. Apostolopoulos, V., et al., The complex immunological and inflammatory network of adipose tissue in obesity. Molecular Nutrition \& Food Research, 2016. 60(1): p. 43-57.

184. Exley, M.A., et al., Interplay between the immune system and adipose tissue in obesity. J Endocrinol, 2014. 223(2): p. R41-8.

185. Mraz, M. and M. Haluzik, The role of adipose tissue immune cells in obesity and low-grade inflammation. J Endocrinol, 2014. 222(3): p. R113-27.

186. Kaufman, C.L., et al., Phenotypic characterization of a novel bone marrowderived cell that facilitates engraftment of allogeneic bone marrow stem cells. Blood, 1994. 84(8): p. 2436-2446.

187. Brestoff, J.R., et al., Group 2 innate lymphoid cells promote beiging of white adipose tissue and limit obesity. Nature, 2015. 519(7542): p. 242-6.

188. Min, B., Spontaneous T Cell Proliferation: A Physiologic Process to Create and Maintain Homeostatic Balance and Diversity of the Immune System. Front Immunol, 2018. 9: p. 547.

189. Min, B., et al., Spontaneous and homeostatic proliferation of CD4 T cells are regulated by different mechanisms. J Immunol, 2005. 174(10): p. 6039-44.

190. Min, B. and W.E. Paul, Endogenous proliferation: Burst-like CD4 T cell proliferation in lymphopenic settings. Seminars in Immunology, 2005. 17(3): p. 201-207.

191. Kieper, W.C., et al., Recent immune status determines the source of antigens that drive homeostatic T cell expansion. J Immunol, 2005. 174(6): p. 3158-63.

192. Ernst, B., et al., The peptide ligands mediating positive selection in the thymus control T cell survival and homeostatic proliferation in the periphery. Immunity, 1999. 11(2): p. 173-81.

193. Morris, D.L., et al., Adipose tissue macrophages function as antigen-presenting cells and regulate adipose tissue CD4+ $T$ cells in mice. Diabetes, 2013. 62(8): $p$. 2762-72.

194. Clements, V.K., et al., Frontline Science: High fat diet and leptin promote tumor progression by inducing myeloid-derived suppressor cells. Journal of Leukocyte Biology, 2018. 103(3): p. 395-407.

195. Limkar, A.R., et al., Frontline Science: Cytokine-mediated developmental phenotype of mouse eosinophils: IL-5-associated expression of the Ly6G/Gr1 surface Ag. J Leukoc Biol, 2020. 107(3): p. 367-377.

196. Wang, G., et al., Targeting YAP-Dependent MDSC Infiltration Impairs Tumor Progression. Cancer Discov, 2016. 6(1): p. 80-95. 
197. Lu, X., et al., Effective combinatorial immunotherapy for castration-resistant prostate cancer. Nature, 2017. 543(7647): p. 728-732.

198. Jung, K., et al., Ly6Clo monocytes drive immunosuppression and confer resistance to anti-VEGFR2 cancer therapy. J Clin Invest, 2017. 127(8): p. 30393051.

199. Singer, K., et al., Differences in Hematopoietic Stem Cells Contribute to Sexually Dimorphic Inflammatory Responses to High Fat Diet-induced Obesity. J Biol Chem, 2015. 290(21): p. 13250-62.

200. Chang, E., M. Varghese, and K. Singer, Gender and Sex Differences in Adipose Tissue. Curr Diab Rep, 2018. 18(9): p. 69.

201. Pettersson, U.S., et al., Female mice are protected against high-fat diet induced metabolic syndrome and increase the regulatory $T$ cell population in adipose tissue. PLoS One, 2012. 7(9): p. e46057.

202. Palmer, B.F. and D.J. Clegg, The sexual dimorphism of obesity. Mol Cell Endocrinol, 2015. 402: p. 113-9.

203. Griffin, C., et al., Sexually dimorphic myeloid inflammatory and metabolic responses to diet-induced obesity. Am J Physiol Regul Integr Comp Physiol, 2016. 311(2): p. R211-6.

204. Su, N., Y. Yue, and S. Xiong, Monocytic myeloid-derived suppressor cells from females, but not males, alleviate CVB3-induced myocarditis by increasing regulatory and CD4(+)IL-10(+) T cells. Sci Rep, 2016. 6: p. 22658.

205. Laparra, A., et al., The Frequencies of Immunosuppressive Cells in Adipose Tissue Differ in Human, Non-human Primate, and Mouse Models. Front Immunol, 2019. 10: p. 117.

206. Azeez, O.I., R. Meintjes, and J.P. Chamunorwa, Fat body, fat pad and adipose tissues in invertebrates and vertebrates: the nexus. Lipids Health Dis, 2014. 13: p. 71.

207. Gesta, S., Y.H. Tseng, and C.R. Kahn, Developmental origin of fat: tracking obesity to its source. Cell, 2007. 131(2): p. 242-56.

208. Highfill, S.L., et al., Bone marrow myeloid-derived suppressor cells (MDSCs) inhibit graft-versus-host disease (GVHD) via an arginase-1-dependent mechanism that is up-regulated by interleukin-13. Blood, 2010. 116(25): p. 573847.

209. Drujont, L., et al., Evaluation of the Therapeutic Potential of Bone MarrowDerived Myeloid Suppressor Cell (MDSC) Adoptive Transfer in Mouse Models of Autoimmunity and Allograft Rejection. PLoS ONE, 2014. 9(6): p. e100013.

210. Deng, T., et al., Class II major histocompatibility complex plays an essential role in obesity-induced adipose inflammation. Cell Metab, 2013. 17(3): p. 411-22.

211. Ostrand-Rosenberg, S., Myeloid derived-suppressor cells: their role in cancer and obesity. Curr Opin Immunol, 2018. 51: p. 68-75.

212. Law, A.M.K., F. Valdes-Mora, and D. Gallego-Ortega, Myeloid-Derived Suppressor Cells as a Therapeutic Target for Cancer. Cells, 2020. 9(3). 


\title{
CURRICULUM VITAE
}

\author{
Katlin Stivers \\ (859)608-1442 \\ katlin@stivers.ws
}

\section{EDUCATION}

- Ph.D. in Microbiology \& Immunology University of Louisville Mentor: James B Hoying

Aug. 2020

Dissertation: Tissue-Resident Myeloid-Derived Suppressor Cells Modulate Immune Homeostasis in Healthy Adipose

○ M.S. in Microbiology \& Immunology

Dec. 2016 University of Louisville Mentor: James B Hoying

- B.A. in Biology and French Wittenberg University

May 2013 GPA: 3.667, Departmental \& University Honors with Distinction

\section{GRANTS}

- American Heart Association Predoctoral Fellowship $2017-2019$ Title: Regulation of Adipose Tissue Inflammation by Resident Myeloid Derived Suppressor Cells

\section{FELLOWSHIPS \& HONORS}

- Excellence in Research Award

University of Louisville Dept. of Microbiology \& Immunology

- Integrated Programs in Biomedical Sciences Fellow University of Louisville

- Kurt J. Fickert Award for Excellence in French Wittenberg University

- Pick and Pen Junior Honorary Wittenberg University 


\section{PROFESSIONAL MEMBERSHIPS}

- Student Member

2018 - Present

Society for Leukocyte Biology

$2016-2019$

- Student Member

$2016-2019$

- Student Member

AAAS (via "Science Program for Excellence in Science")

- Student Faculty Liaison

$2016-2017$

Microbiology \& Immunology Student Organization

- Secretary/Treasurer

Microbiology \& Immunology Student Organization

$2015-2016$

\section{PROFESSIONAL EXPERIENCE}

\section{LABORATORY EXPERIENCE}

- Graduate Research Fellow

University of Louisville Dept. of Microbiology \& Immunology

Aug. 2014 - Present

\section{Advisor: James B. Hoying}

- Flow cytometry: multiparametric panel design; surface \& intracellular staining; cytometer operation/maintenance; data analysis (FlowJo)

- Cell sorting: FACS \& magnetic bead sorting

- Cell proliferation assays: cytometry based \& MTT colorimetric

- Primary cell isolation \& culture: adipose tissue stromal cells \& splenocytes

- Gene expression analysis: RNA isolation (cells \& whole tissue); primer design; qRT-PCR; gel electrophoresis

○ Mouse handling \& colony husbandry

- ELISA: cell culture supernatants

- Immunohistochemistry: whole mount tissue staining

- Confocal microscopy: whole mount adipose tissue

- Experimental Design: stromal cell isolation protocol design \& development; design \& optimize multiparametric staining panels for analysis of adipose stromal cells.

- Lab Administration: budget preparation \& management

Advisor: David P. Stirling (6-week rotation)

- Introduction to two-photon microscopy: sample preparation; tissue staining; image analysis 
Advisor: Nejat K. Egilmez (6-week rotation)

- Bacteria culture techniques

- PCR and gel electrophoresis: mouse phenotyping; bacterial strain identification

- Laboratory Technician

University of Kentucky Dept. of Anatomy \& Neurobiology

May - Sept. 2013

Advisor: Don M. Gash

- Radioactive techniques: autoradiography \& gamma counting on radio-labeled rat tissue

- Microscope imaging: light microscope analysis of dopamine production in NHP brain tissue sections

- Laboratory Technician

May - Aug. 2012

University of Kentucky Dept. of Anatomy \& Neurobiology

Advisor: Don M. Gash

- Histology \& Immunohistochemistry: non-human primate tissue sectioning \& fixation; H\&E, Nissl, \& dopamine staining

- Lab Administration: preparation \& troubleshooting "Good Lab Practices" Standard Operation Procedures

- Undergraduate Researcher

Wittenberg University

Spring 2011

\section{Coursework for "Survey of Human Disease"}

- Transmission Electron Microscopy: labeling, imaging, \& analysis of rat hepatocytes

○ Fluorescent Microscopy: labeling, imaging, \& analysis of rat hepatocytes

\section{TEACHING EXPERIENCE}

○ Student Instructor

$2017 \& 2018$

University of Louisville

Microbiology \& Immunology Methods: Flow Cytometry

$\circ \quad$ English Language Assistant (T.A.P.I.F.) Lycée Jean Renou \& Collège Paul Esquinance, La Réole, France

Sept. 2013 - May 2014

○ French 101 \& 102 Supplemental Instructor Wittenberg University

Aug. 2012 - May 2013 


\section{LANGUAGE EXPERIENCE}

- English language editing for non-native speakers: scientific manuscripts for publication \& "Good Lab Practices" SOPs.

- López-Mercado, J., Nambo, A., Toribio-Nava, M., et al. "High and low esterification degree pectins decomposition by hydrolysis and modified Maillard reactions for furfural production." Clean Technologies and Environmental Policy: Focusing on Technology Research, Innovation, Demonstration, Insights, and Policy Issues for Sustainable Technologies, 2018, 20(7): 14131422.

- French to English Translation: 40 of 99 vignettes in Exercises du Style by Raymond Queneau (approx. 5000 words) for undergraduate honors thesis

○ Fluent in French: 13 years of formal training in written \& spoken French

- Language Tutoring: middle school, high school, \& undergraduate level

\section{RESEARCH ACTIVITIES}

\section{PEER REVIEWED ORIGINAL PUBLICATIONS}

1. Stivers, K.B., Chilton, P.M., Beare, J.E., Dale, J.R., Alard, P., LeBlanc, A.J., \& Hoying, J.B. "Adipose-resident myeloid-derived suppressor cells modulate immune cell homeostasis in healthy mice." Immunology and Cell Biology. 2020. [In Press]

2. Omer, L., Hindi, L., Militello, G., Stivers, K.B., Tien, K.C., \& Boyd, N.L. "Familial hypercholesterolemia class II low-density lipoprotein receptor response to statin treatment" Disease Models \& Mechanisms, 2020, 13(4): dmm042911.

\section{INVITED REVIEWS}

1. Stivers, K.B., Beare, J.E., Chilton, P.M., Williams, S.K., Kaufman, C.L., \& Hoying, J.B. "Adipose-derived cellular therapies in solid organ and vascularizedcomposite allotransplantation." Current Opinion in Organ Transplantation, 2017, 22(5): $490-498$.

\section{ABSTRACTS}

1. Stivers, K.B., Chilton, P.M., Beare, J.E., Dale, J.R., \& Hoying, J.B. 2018. Adipose-Resident Myeloid-Derived Suppressor Cells Modulate Immune Homeostasis in Healthy Mice. Poster. Society for Leukocyte Biology 2018, 14 October 2018, Chandler, Arizona. 
2. Stivers, K.B., Chilton, P.M., Beare, J.E., Dale, J.R., \& Hoying, J.B. 2017. Tissue-Resident Myeloid-Derived Suppressor Cells in Adipose from Lean Mice. Poster. Society for Leukocyte Biology $50^{\text {th }}$ Annual Meeting, 7 October 2017, Vancouver, B.C.

3. Stivers, K.B., Chilton, P., Beare, J.E., Dale, J.R., Kaufman, C.L., and Hoying JB. 2017. Myeloid-Derived Suppressor Cells in Adipose Tissue Homeostasis. Poster. Experimental Biology 2017, 25 April 2017, Chicago, Illinois.

4. Stivers, K.B., Chilton, P., Beare, J.E., Dale, J.R., Kaufman, C.L., Williams, S., and Hoying, 2016. Elucidating the Role of Myeloid-Derived Suppressor Cells in Maintaining Tissue Homeostasis. Poster, 2016 Research! Louisville, Louisville, Kentucky, October 2016.

5. Stivers, K.B., Chilton, P.M., Beare, J.E., Dale, J.R., and Hoying, J.B. 2015. Myeloid Derived Suppressor Cells within Adipose from Lean, Healthy, Mice. Poster, 2015 Research! Louisville, Louisville, Kentucky, October 2015.

\section{SELECTED TALKS}

1. Stivers, K.B., Chilton, P.M., Beare, J.E., Dale, J.R., \& Hoying, J.B. 2018. Adipose-Resident Myeloid-Derived Suppressor Cells Modulate Immune Homeostasis in Healthy Mice. Flash talk. Society for Leukocyte Biology 2018, 14 October 2018, Chandler, Arizona.

2. Stivers, K.B., Chilton, P.M., Beare, J.E., Dale, J.R., \& Hoying, J.B. 2017. TissueResident Myeloid-Derived Suppressor Cells in Adipose from Lean Mice. Selected speaker: "Metabolism in Leukocyte Memory", Society for Leukocyte Biology $50^{\text {th }}$ Annual Meeting, 7 October 2017, Vancouver, B.C.

3. Stivers, K.B., Chilton, P., Beare, J.E., Dale, J.R., Kaufman, C.L., and Hoying JB. 2017. Myeloid-Derived Suppressor Cells in Adipose Tissue Homeostasis. "Kaley Lecture Featured Topic" selected speaker: "The Complications of Diabetes and the Role of Inflammation: Mechanisms and Therapeutic Opportunities" Session of Experimental Biology 2017, 25 April 2017, Chicago, Illinois. 